\title{
OBESITY AND ITS IMPACT ON A PROVINCIAL. HEALTH SYSTEM IN CANADA
}




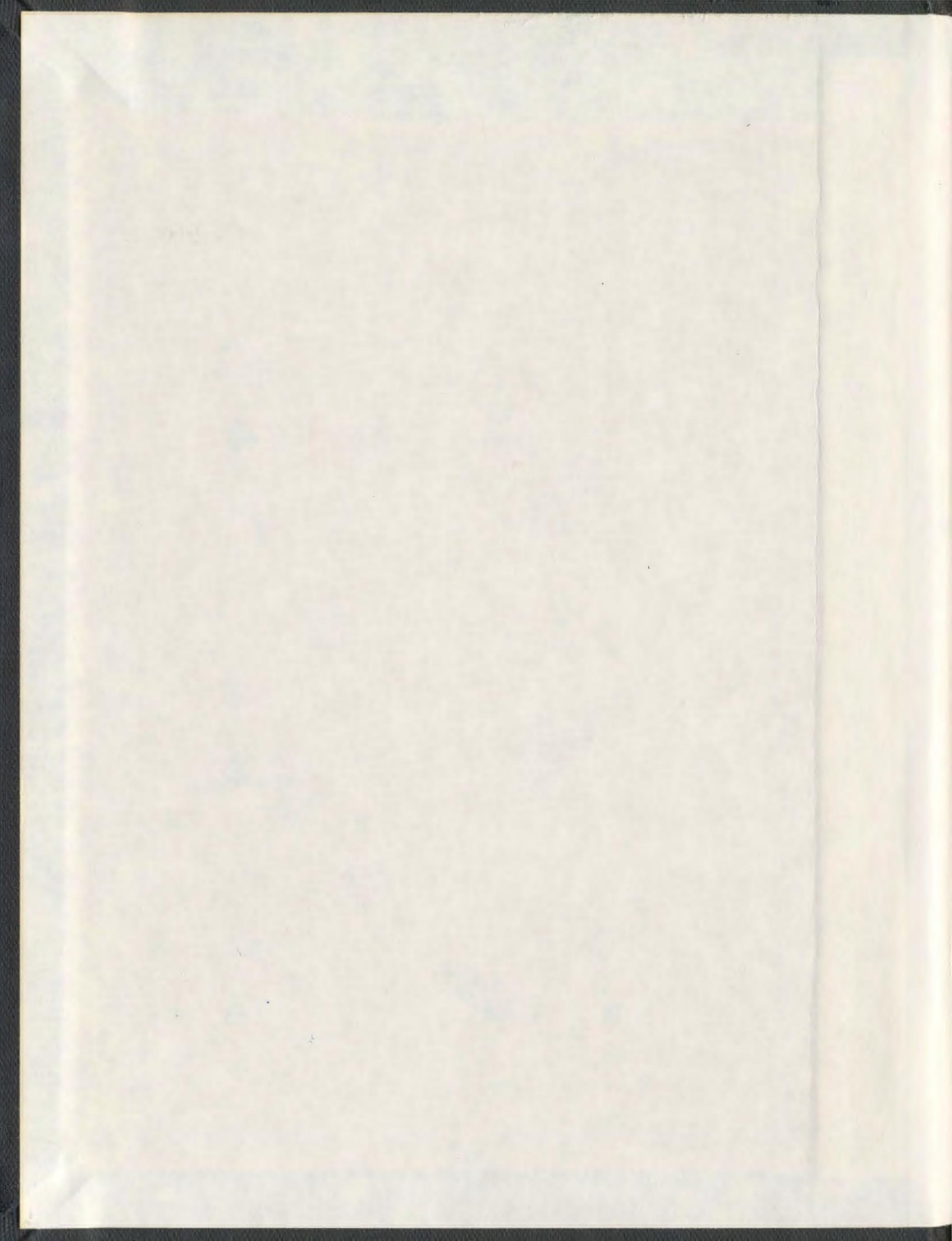


001311

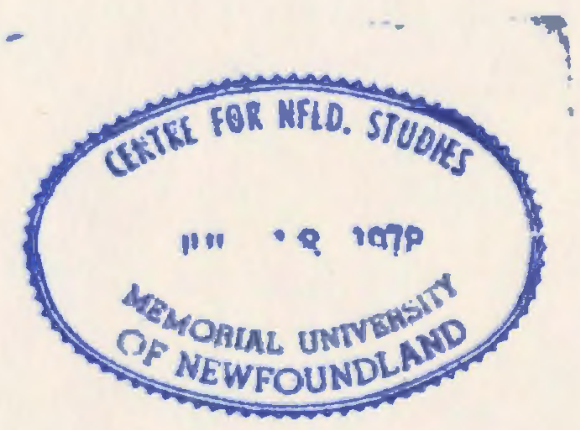



OBESITY AND ITS IMPACT ON A PROVINCIAL HEALTH SYSTEM IN CANADA

\author{
By
}

Laurie Kathleen Twells BA, M.Sc.

A thesis submitted to the School of Graduate Studies in partial fulfillment of the requirements for the degree of Doctor of Philosophy

Faculty of Medicine

Memorial University

2008

St. John`s

Newfoundland 


\begin{abstract}
Purpose: The main focus of this study is to explore the relationship between body mass index (BMI) as a reflection of obesity, its association with chronic discase, health serviees utilization and its impact on direct costs to the health system, in the province of Newfoundland and Labrador.

Methods: In a secondary analysis of the provincial component of the Canadian Community Health Survey version 1.1 (2000/01), survey records for respondents aged 20-64 ( $\mathrm{n}=2345)$ were linked to provincial physician and hospitalization data in order to obtain objective measures of longitudinal health services utilization. Regression models were used to examine whether BMI level was an independent predictor of family physician (GP) visits, hospitalization and costs.

Results: Of the study sample $37 \%, 39 \%$ and $17 \%$ and $6 \%$ were classified as nomal, overweight, obese, and morbidly obese respectively. The obese (including morbidly obese) were more likely to report having serious chronic conditions after adjusting for age and gender. Analyses identified the morbidly obese group (BMI $\left.>35 \mathrm{~kg} / \mathrm{m}^{2}\right)$ as having a significantly higher number of GP visits over a 5 -year period when compared with the nomal weight group (BMI $18.5-24.9 \mathrm{~kg} / \mathrm{m}^{2}$ ), [median 22.0 vs. 17.(), p<.()5], and as having significantly higher average costs of GP $(\mathrm{p}<.001)$ and specialist services $(\mathrm{p}<.05)$. Controlling for number of chronic conditions and other covariates, being morbidly obese remained a significant independent predictor of family physician visits ( $p$ $\because .001)$ and total physician costs $(\mathrm{p}<.01)$, but was not a predictor of hospitalization.

Conclusions: Morbid obesity is independently associated with increased GP utilization and total physician costs but not with hospital utilization. Some future health promotion/education and weight loss interventions should be targeted at this high-risk group; however a population health approach must be developed in order to have an impact on the prevalence of obesity in this population.
\end{abstract}




\section{ACKNOWLEDGEMENTS}

I would like to express my genuine appreciation and thanks to everybody who supported me during my graduate program.

I acknowledge the assistance provided by the members of my Supervisory Committec, Drs Brendan Barrett, Patrick Parfrey, Barbara Rocbothan and Tracey Bridger. A special thank you goes to my supervisor Dr. Brendan Barrett who provided me with direction throughout the project.

I would like to acknowledge the Newfoundland and Labrador Centre for Applied Health Research for funding this research project and Don MeDonald of the Newfoundland and Labrador Centre for Health Information for allowing me to complete this work and become "onc of them" for a short while.

The completion of this research project owes much to the support of both Dr. Patrick Parfiey who encouraged me to enter the program and Dr. Stephen Bornstein whom I had the privilege of working with through the early part of my rescarch. Thank you.

I would also like to acknowledge two fellow PhD students: Debbie Gregory who provided me with support and guidance throughout the program, a huge thank you, and to John Knight who assisted me in the technical aspeets of this research. I an indebted to you for passing on to me your knowledge of database linkages. Additionally, I would like to thank a friend Helen Knapman and my mother Brenda Tumer for their patience and editorially support during the last stages of my thesis. 
I dedicate this dissertation to my parents Brenda and Neil Turner and my sister Lesley and her husband Jamie, all who have provided me with endless support in everyway throughout my academic endeavors and have always believed that I could and would aceomplish what I set out to do; to my brother Mark who taught me that everyday is 'perfect'. To my husband Alex who has always been there for me and who understands this topic as well as I do. Alex our long conversations and productive debates kept me focused and motivated these last 6 years. Last but not least to my children Louise, Jack and Amelia who have wondered why it has taken me so long to write this "book". 


\section{TABLE OF CONTENTS}

ABSTRACT

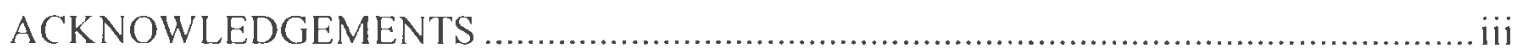

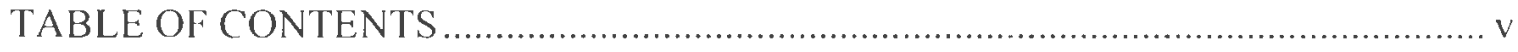

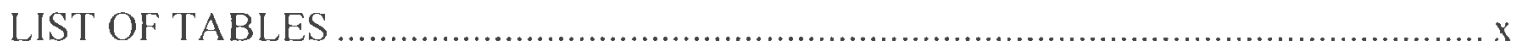

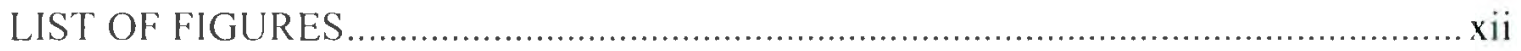

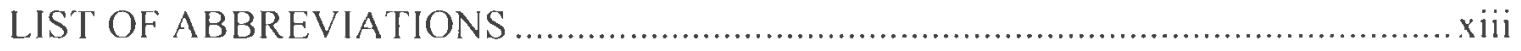

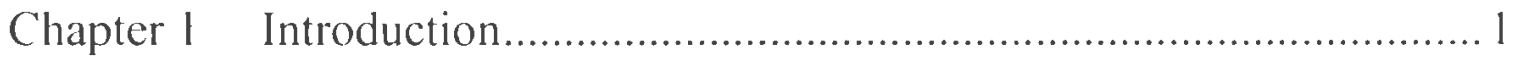

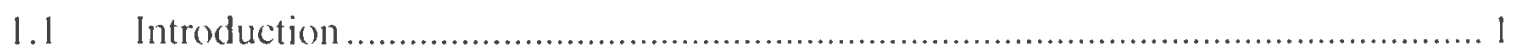

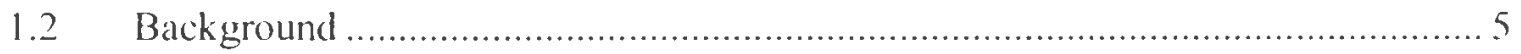

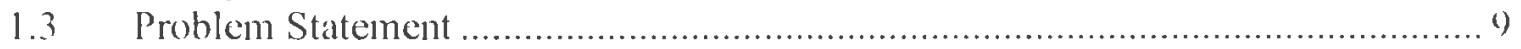

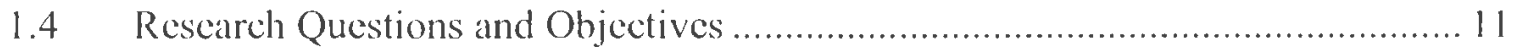

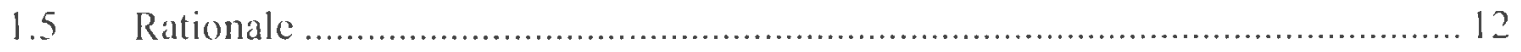

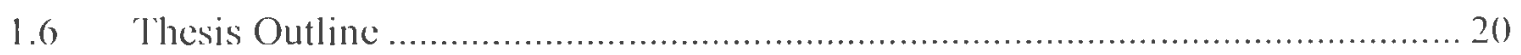

Chapter 2 Theoretical Model and Literature Review................................21

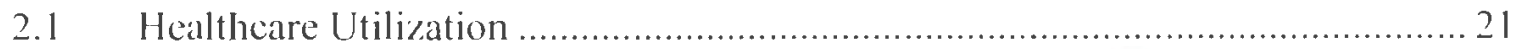

2.2 Andersen`s Behavioral Model of Health Services Utilization .......................... 23

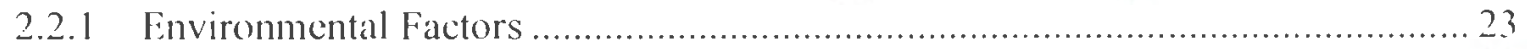

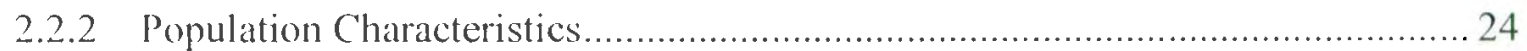

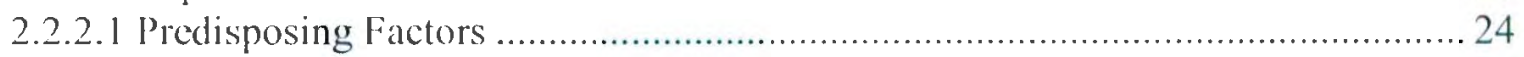

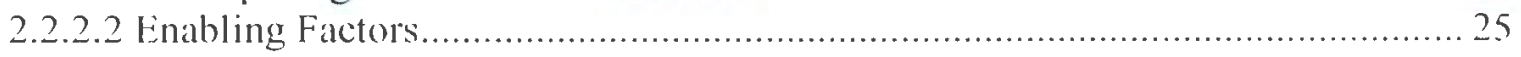

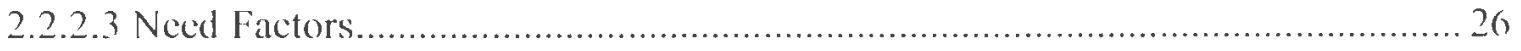

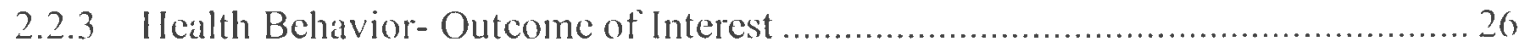

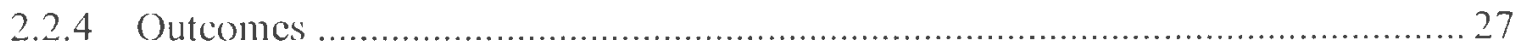

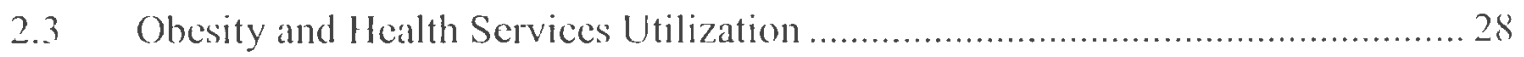

2.3.1 Obesity and Visits to a Primary Care Provider ......................................... 29)

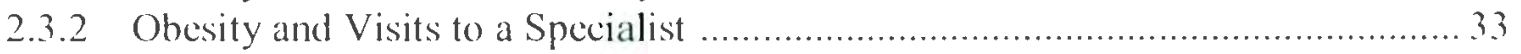

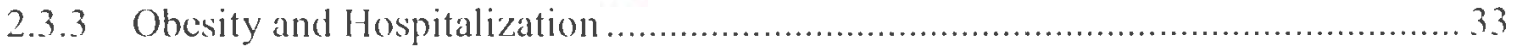

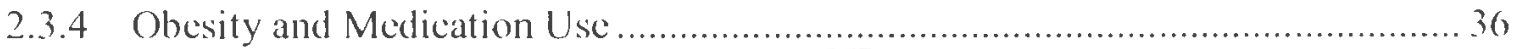

2.4 Obesity and the Behavioral Model of Health Services Utilization ......................37

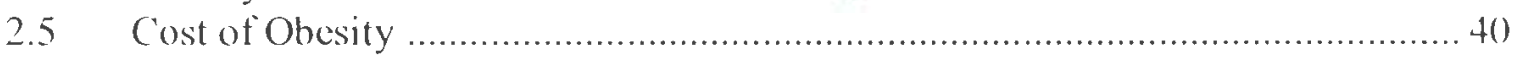

2.5.1 Prevalence-based Approach .................................................................. 41

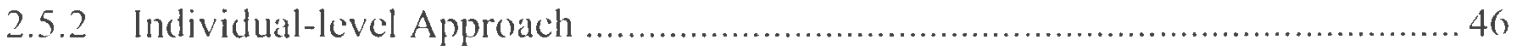




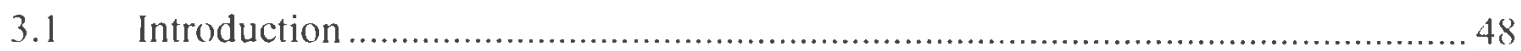

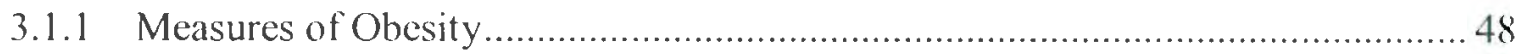

3.1.2 How is Body Fat Measured? .

3.1.2.1 Direct Measurements of Body Fat ............................................................. 50)

3.1.2.2 Indirect Measurements of Body Fat ............................................................

3.1.3 Body Mass Index and Associated Health Risk ...............................................54

3.1.4 Body Mass Index as a Measure of Body Fat.................................................55

3.1.5 Health Canada and Changing BMI Guidelines ...............................................56

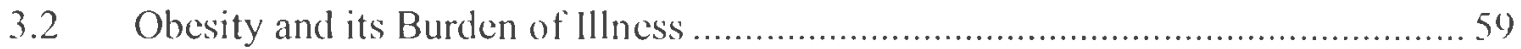

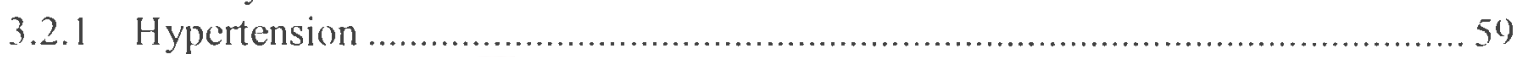

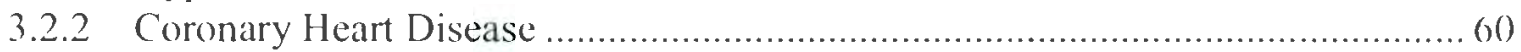

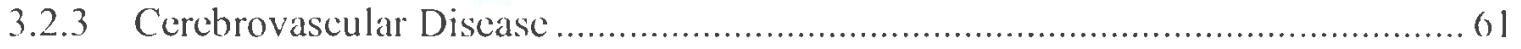

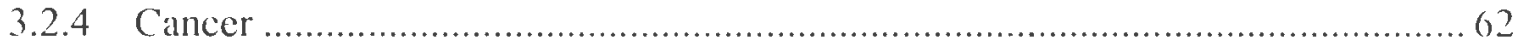

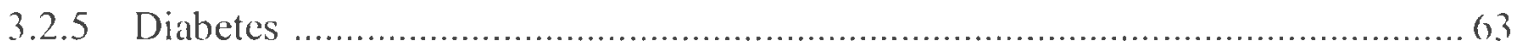

3.2.6 Endocrine and Metabolic Disturbances ............................................................. 64

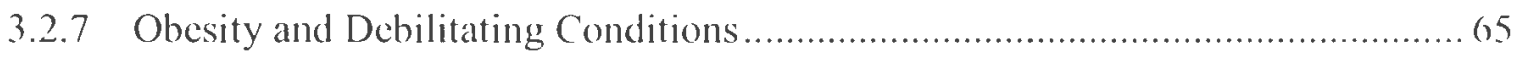

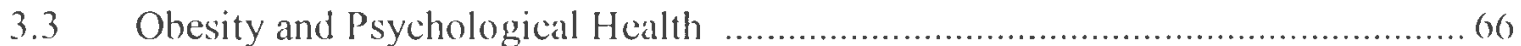

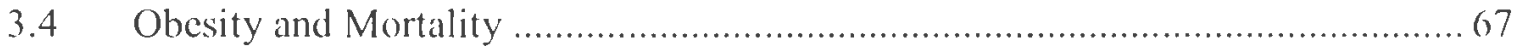

3.5 Obesity and Disease - a Causal Relationship? ............................................... (69)

3.6 Can Overweight and Obese Individuals be Ilealthy?...................................... 70

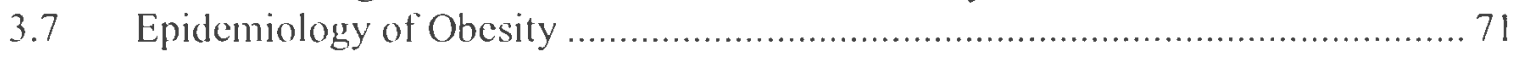

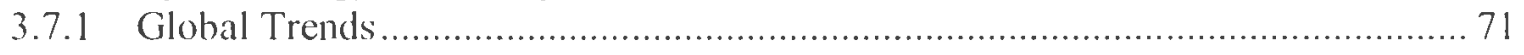

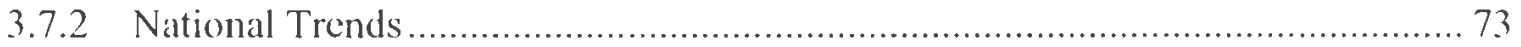

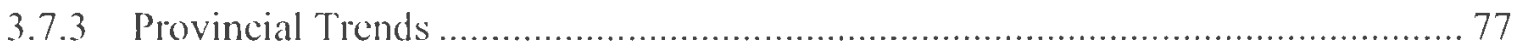

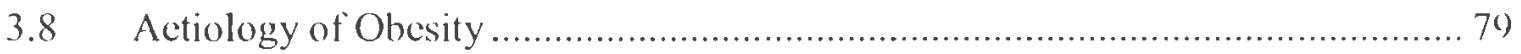

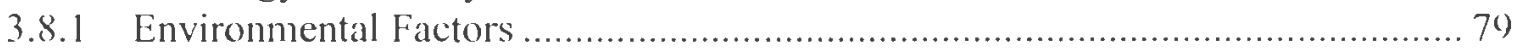

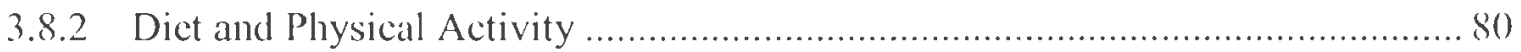

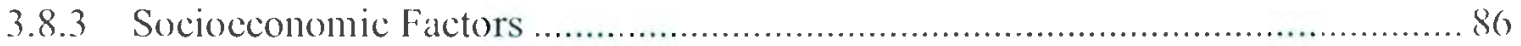

3.9 The Geneties and Medical Causes of Obesity ................................................ 87

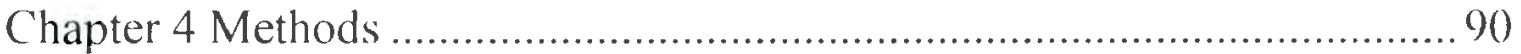

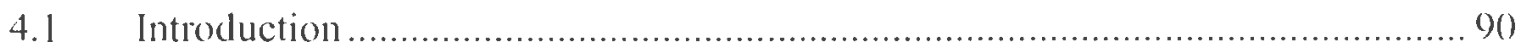

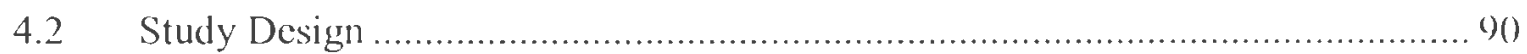

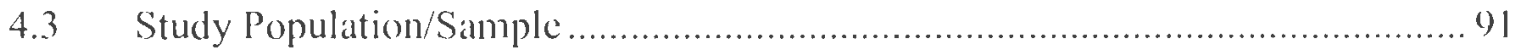

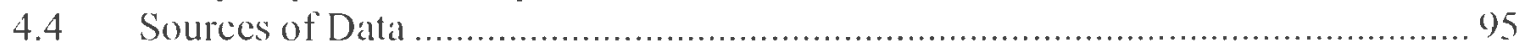

4.4.1 The 2000/2001 Canadian Community Health Survey ......................................95

4.4.1.1 Aceess to Survey Data .............................................................................. 97

4.4.2 Medical Care Plan Database ……….................................................................99

4.4.3 Clinical Database Management System .................................................. 102

4.5 Database Linkage Procedure................................................................. 102 
4.6 Measures in the Current Study .............................................................. 102

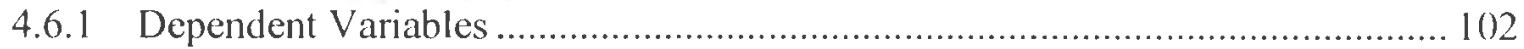

4.6.2 Independent Variables................................................................................. 105

4.6.2.1 The Primary Relationship under Study ......................................................... 105

4.6.2.2 Selection of Independent Variables from the Survey ..................................... 106

4.7 Comparison of Subjective and Objective Visits to a Physician .......................... 108

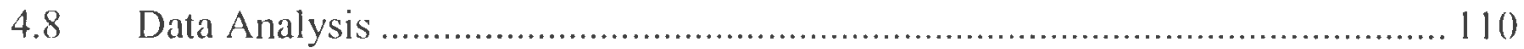

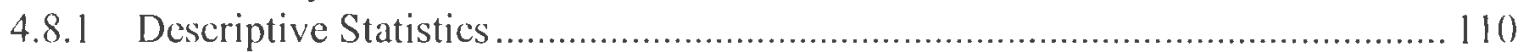

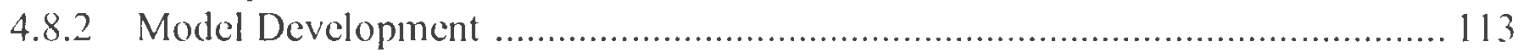

4.8.2.1 Logistic Regression Models .................................................................... 114

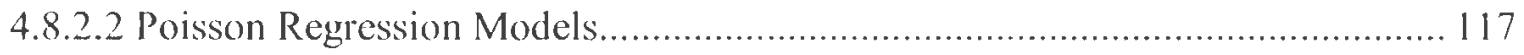

4.8.2.3 Multiple Linear Regression Models .......................................................... 120

4.9 Chronic Disease as a Mediator Variable...................................................... 123

4.10 Ethical Considcrations ......................................................................... 128

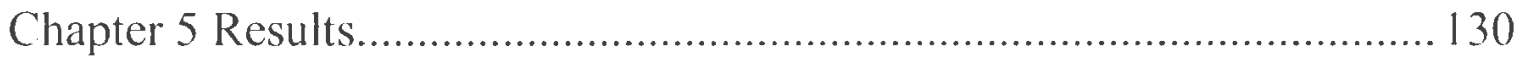

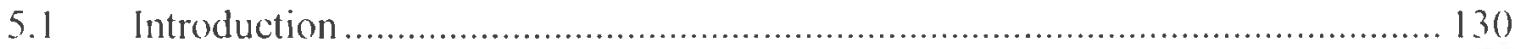

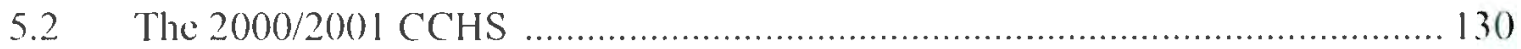

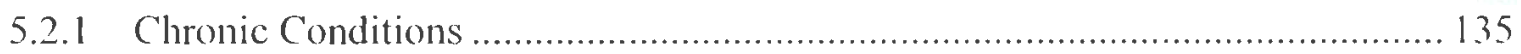

5.2.2 Self Reported Health Serviees Utilization ................................................... 137

5.2.3 Logistic Estimates: Associations with Obesity ……..................................... 139

5.2.4 Obesity as a Predictor of Chronic Discase .................................................. 141

5.3 Measures of Actual Health Services Utilization .............................................. 14.3

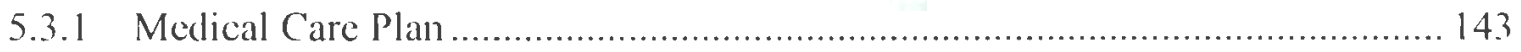

5.3.2 Descriptive Statistics: Hospital Separations.................................................. 147

5.4 Comparison of Self Report and Actual Visits to a Physician .......................... 149)

5.5 Poisson Prediction Models ...................................................................... 152

5.5.1 Poisson Estimates for Actual Measures of Health Services Utilization ............. 152

5.5.1.1 Dependent Variable: Actual Number of Visits to a GP ................................... 15.3

5.5.1.2 Dependent Variable: Actual Number of Visits to a specialist ............................ 153

5.5.1.3 Dependent Variable: Actual Number of Visits as an In-P'atient ...................... 154

5.5.1.4 Dependent Variable: Total Length of Stay in Hospital................................... 158

5.5.1.5 Dependent Variable: Number of Surgical Day Care visits ............................... 158

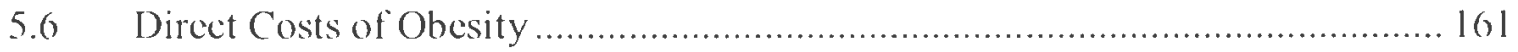

5.6.1 Descriptive Cost Analysis ........................................................................ 162

5.6.2 Multiple Regression Cost Estimates ............................................................. 167

Chapter 6 Discussion and Conclusions ............................................... 171

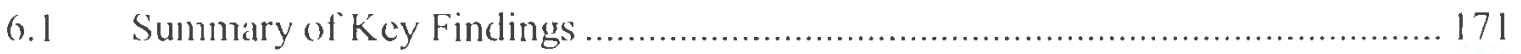

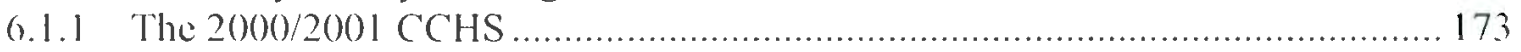




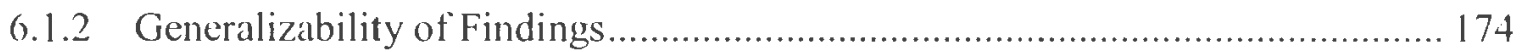

6.1.3 Obesity and Actual Visits to a Primary Care Provider .................................... 174

0.1.4 Obesity and Hospitalization ......................................................................... 176

6.1.5 Obesity and Andersen's Model of Health Services Utilization ........................ 178

6.1.6 Obesity and Direct Healtheare Costs .................................................. 179

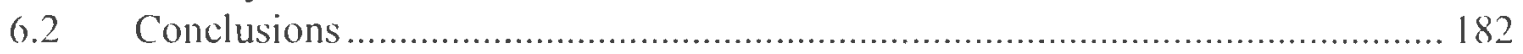

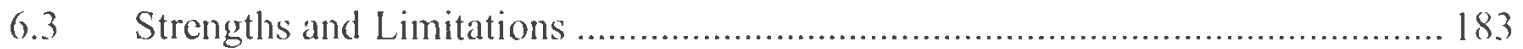

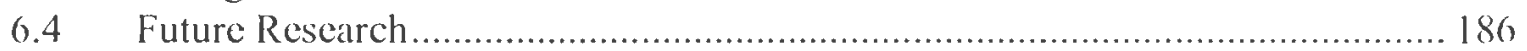

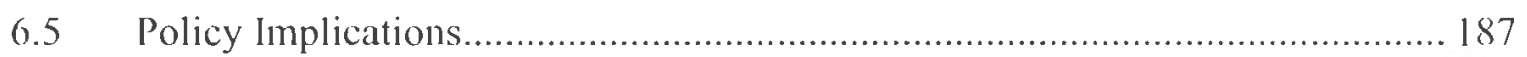

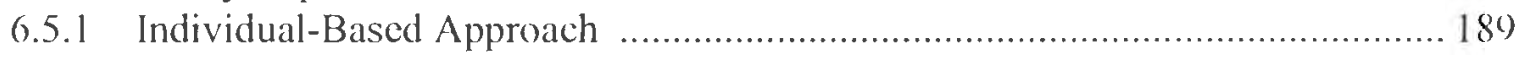

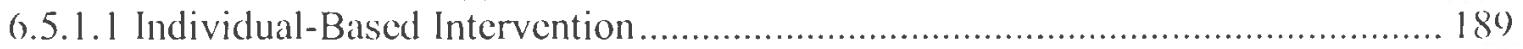

0.5.1.2 Challenges and Opportunities to the Individual-Based Approach .................. 191

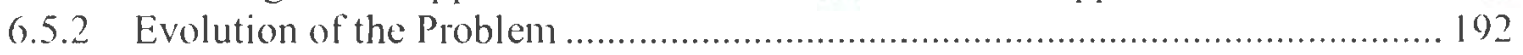

6.5.2.1 Population Health Approach .................................................................. 19.

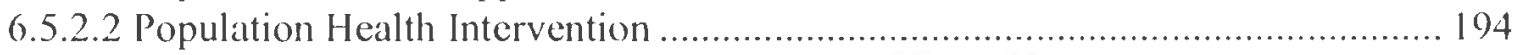

6.5.2.3 Challenges and Opportunities to the Population Health $A$ pproach .................. 196

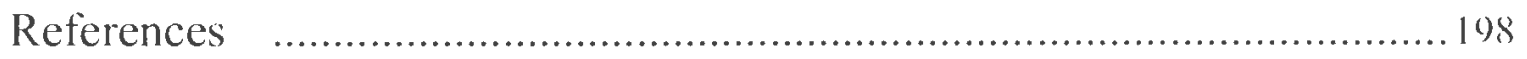

\section{Appendices}

Appendix A - Andersen's Behavioral Model of Health Services Utilization .............. 216

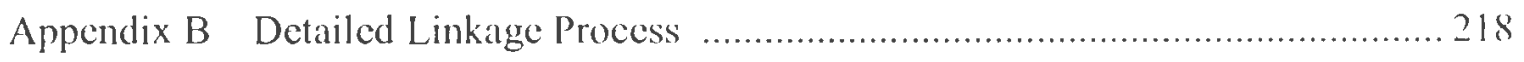

Appendix C 2000/2001 CCHS Variables used in the Current Study....................... 222

Table la Select Variables Measuring Health Bchavior ...................... 223

Table 1 b Select Variables Measuring Population Characteristics ........ 224

Appendix D Does Body Mass Index Change overtime ...................................... 226

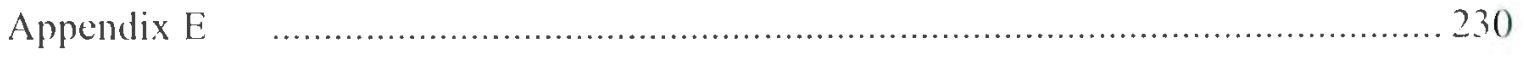

Table 1: Distribution of Physicians in NL 1099/20000 ....................231

Table 2: Analysis of Visits to a Physician 1998-2003 by lFFS Fee-code.......232

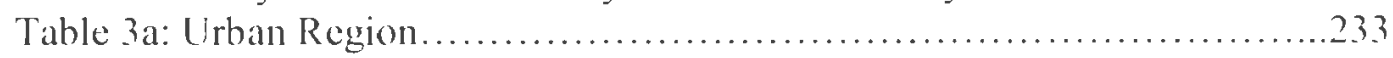

Table 3b: Rural Region............................................. 233

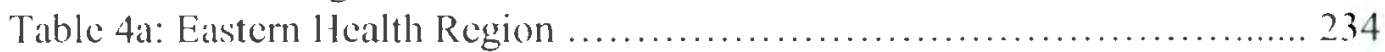

Table 4b: Central Health Region .......................................... 234

Table 4c: Western Health Region .......................................234

Table 4d: Northern Health Region .......................................234 


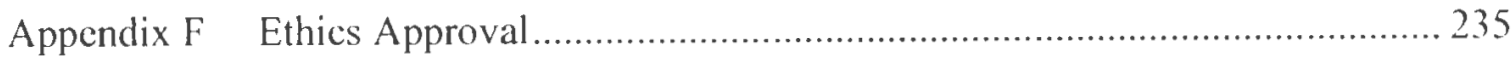

Appendix G Permission from the Department of Health and Community Services .. 238

Appendix H Permission from the NL Centre for Health Information ...................... 240

Appendix I Permission from the Medical Care Plan............................................ 243

Appendix J Poisson Models 1a-2c Actual Physician Utilization ............................. 245

Appendix K Poisson Models 3a-5c Actual Hospital Utilization ................................. 252

Appendix L Multiple Regression Models 6a-7c Physician \& Hospital Costs ........... 261 


\section{LIST OF TABLES}

Table 3.1 BMI and Associated Health Risks .................................................... 49

Table 3.2 Comparison of BMI Classification Systems 1998-2003...............58

Table 3.3 The Prevalence of Adult Obesity in Canada hased on Self Reported Heights and Weights 1985-2005 _................................................. 75

Table 3.4 The Prevalenee of Adult Obesity in Canada by provinee based on Self Reported Heights and Weights 1995-2005 ....................... 78

Table 4.1 FFS Physician Specialty Codes and Specialty Description .................. 100

Table 4.2 Independent and Dependent Variables used in Primary Analysis ......... 107

Table 5.1 CCHS Demographies by Level of BMI ........................................ 132

Table 5.2 Lifestyle Characteristics and Health Status Variables by Level of $\mathrm{BMI}$

Table 5.3 Self-Reported Prevalenee of Chronic Conditions by Level of BMI ..... 136

Table 5.4 Subjective Health Care Resouree Use Indicators in Previous 12 Months by Level of BMI

Table 5.5 Logistic Regression: Associations with Morbid Obesity and Obesity... 140

Table 5.6 Logistic Regression: Level of BMI as a Predictor of Self-Reported Chronic Discase

Table 5.7 Actual Physician Health Services Utilization by Level of BMI 145

Table 5.8 Hospital Health Services Utilization by Level of BM1 148

Table 5.9 Comparison of Self Report and Actual Visits to al Physician 151

Table 5.10 Poisson Regression Estimates $(\beta)$ and Standard Errors (SE), Dependent Variable: Actual Number of Visits to a GP 1998-2003 155

Table 5.11 Poisson Regression Estimates $(\beta)$ and Standard Errors Dependent Variable: Actual Number of Visits to a specialist Doctor 1008-200)3 ... 150 
Table 5.12 Poisson Regression Estimates $(\beta)$ and Standard Errors Dependent Variable: Actual Number of Visits as an In-Patient 1998-2003

Table 5.13 Poisson Regression Estimates $(\beta)$ and Standard Errors Dependent Variable: Actual Length of Stay (LOS) in Hospital 1998-2003

Table 5.14 Poisson Regression Estimates $(\beta)$ and Standard Errors Dependent Variable: Actual Surgical Day Care Visits 1998-2003 160

Table 5.15 Summary of Average 5-Year Physician and Hospital Costs by BMI .... 164

Table 5.16 Multiple Regression Estimates $(\beta)$ and Standard Errors (SE) Dependent Variable: Total Costs of Physician Services 1998-2003 ..................... 169

Table 5.17 Multiple Regression Estimates $(\beta)$ and Standard Errors (SE) Dependent Variable: Total Costs for in-Patient Hospital Services 1998-2003 170 


\section{LIST OF FIGURES}

Figure 3.1 Global Prevalence of Obesity in Adult Populations $\left(\mathrm{BMl} \geq 30 \mathrm{~kg} / \mathrm{m}^{2}\right)$ in OECD Member Countries.

Figure 3.2 Components of Total Energy Expenditure in Sedentary and Active Persons

Figure 4.1 Flowchart of the Selection of Study Sample ........................................ 94

Figure 4.2 Total Fec-For-Service Visits 1998-2003 ....................................... 112

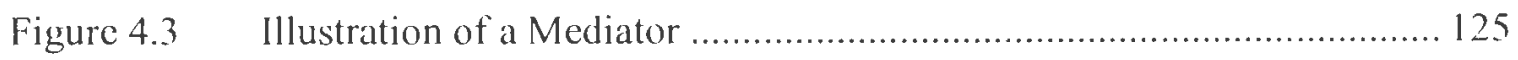

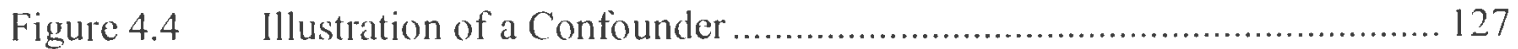

Figure 5.1 Physician Utilization (GP and specialist) by level of BMI........... 46

Figure 5.2 Physician Costs by Level of BMI 1998-2003 ............................... 165

Figure 5.3 Hospital in-patient Costs by Level of BMI 1998-2003 .................... 160 


\section{LIST OF ABBREVIATIONS}

Adult Treatment Panel (ATP)

Body Mass Index (BMI)

Canadian Community Health Survey (CCHS)

Canadian Database Management System (CDMS)

Canadian Institute for Health Information (CIHI)

Cardiovascular Disease (CVD)

Chronic Conditions (CC)

Coronary Heart Discase (CHD)

Cost-of-Illness (COI)

Cost per Weighted Case (CPWC)

Discharge Abstract Database (DAD)

Fee-for-Service (FFS)

Health Maintenance Organization (HMO)

Health Services Utilization (HSU)

Intemational Obesity Task Force (IOTF)

International Classification of Diseases (ICD)

Medical Care Program (MCP)

National Health and Nutrition Examination Survey (NHANES)

Newfoundland and Labrador (NL)

Newfoundland and Labrador Centre for Health Information (NLCHI)

Organization for Economic Cooperation Development (OECD) 
Population Attributable Fraction (PAF\%)

Population Attributable Risk (PAR)

Provincial Mortality Surveillance System (PMSS)

Statistical Package for Social Sciences (SPSS)

The World Health Organization (WHO)

Years of Life Lost (YLL) 


\section{CHAPTER 1 Introduction}

\subsection{Introduction}

In 2005, most industrialized countries spent on average $8.6 \%$ of their Gross Domestic Product (GDP) on healtheare services. During this time Canada spent $9.9 \%$ compared to $7.7 \%$ in the United Kingdom, 10.1\% in France, and 15\% in the United States. In most of

these countries, the proportion of GDP spent on healtheare has been rising steadily since the 1980's and continues to rise. Similar trends have been noted in Germany, Australia and New Zealand. The main drivers of increased expenditure in healtheare include new technologies, drug therapies and increasing consumer demand or utilization.' Within these expenditures, a large proportion is directed towards the increasing prevalence of chronic illness and its management. ${ }^{2}$

In 2007, according to the Canadian Institute for Health Information (CIHI), an independent pan-Canadian, not-for-profit organization, total healtheare expenditure by use of funds was categorized as follows. The majority of public expenditures were directed towards: hospitals (28.4\%), physicians (13.4\%), drugs (10.4\%), and other health professionals (10.8\%) while the remaining funds were directed towards capital, public health, administration and other institutions. ${ }^{3}$

Chronic conditions have been identified as a key challenge for healtheare in the $21^{\text {t }}$ century. According to the World Health Organization (WHO), chronic discases are the major cause of death and disability worldwide and are reaching epidemic proportions in both developed and underdeveloped eountries. Non communicable discases have surpassed communicable diseases in developed countries as the most serious problem for 
governments in this century; once diagnosed they become a daily, ongoing concern for the individual, the community and the provision of health services. Chronic conditions affect a significant proportion of the population and range from allergies and skin disorders to recurrent depression and cardiovascular disease, diabetes, congestive heart failure, hypertension, renal failure and chronic obstructive pulmonary disease. Chronic diseases are projected to account for $89 \%$ of all deaths by year 2015 in Canada. ${ }^{2}$

According to Health Canada and based on the 1998 Economic Burden of Illness Report, the Cost of Illness (COI) in Canada was approximately $\$ 160$ billion. Direct costs and indirect costs accounted for $52.7 \%$, and $43.3 \%$, respectively. ${ }^{4}$ Direct costs reported in monetary terms are defined as the value of goods and services for which payment was made for the following: treatment, care and rehabilitation, hospital expenditures, drugs, physician services, other institutions, other professionals, public health and health research. Indirect costs are defined as the estimated value of economic output lost because of illness, sick leave, absence from work, disability or premature death. ${ }^{5}$ In 2007 , the Public Health Agency of Canada estimated that up to $67 \%$ of direct healthcare costs were spent on chronic conditions. ${ }^{6}$

Based on this report, the top five most costly diseases in Canada are predominantly chronic in nature. The top five in rank order were: cardiovascular diseases, musculoskeletal diseases, cancer, injuries and respiratory diseases. Cardiovascular diseases have consistently represented the number one category. In Newfoundland and Labrador (NL), the comparable figure for COI was approximately $\$ 3.1$ billion with the order of diseases being slightly different with the addition of diseases of the digestive 
system in place of injuries. The top five chronic diseases in NL included; musculoskeletal conditions (12.9\%), cardiovascular diseases $(12 \%)$, cancers $(9 \%)$, respiratory diseases $(5.3 \%)$ and diseases of the digestive system (3.7\%).

As a result, research on the utilization of healthcare services and factors predicting health services utilization is of increasing value to governments, health boards and hospital administrators because of the high and rising expenditures in healthcare and the limited resources available. Providing this information to policy-makers and decisionmakers will aid in decision-making concerning the emerging health needs of the population and the changing patterns of the population's health services use. This information will help to assist in improved planning and a more efficient allocation of scarce resources through the provision of services and the development of health and social policies.

It is well known that health services utilization results from a complex, interrelated set of factors, and many models have been developed to aid in the identification of these factors and explain how they impact healthcare utilization..$^{7-9}$ In the early 1960's, Ronald Andersen developed a conceptual Behavioral Model of Health Services Utilization, with the goal of identifying factors that influenced the use of healthcare services. This seminal work was conducted in the United States and was meant to assist in the analysis of national survey data collected by the Centre for Health Administration Studies, University of Chicago. Interestingly, Andersen's initial framework was developed in response to what policy-makers felt was an underutilization of healthcare services in the United States by certain sub-groups of the population. It was hoped that this model would 
enlighten policy-makers as to what factors determined whether an individual would seck health services and, was meant to guide the development of policies to increase health services utilization in certain sub-groups, given that cost was not the concern that it is today.

Andersen`s initial model was later modified by many other researchers, but the most commonly used adaptation was by Andersen \& Newman and published in 1973. ${ }^{\mathrm{X}}$ This model suggests that in the context of a particular Environment and Health System, health services utilization is determined by Population ('haracteristics classified as three main types: predisposing factors, enabling factors and need factors. According to the author, in order for the use of health services to take place (1) an individual must be predisposed to receive medical care (2) there must be in place enabling conditions that allow the individual to obtain health serviees and (3) the individual must pereeive a need for these services. Predisposing factors include variables such as gender, age and social status. Enabling factors include conditions that facilitate or inhibit the use of physician services, e.g. the distance to the health centre, the type of municipality, working time, family size and health insurance coverage. Necd variables include the presence or absence of chronic diseases, disability days, illness conditions and psychological well-being. According to this model a combination of these factors influenees an individual's Health Behavior or more specifically their use of health services. ${ }^{8}$ More recently, personal health choices have been included in the use of health services (e.g., the decision to take part in screening initiatives for cancer)." 
Over the last 40 years, this model has been tested, modified and adapted by researchers who have set out to better understand the complexity around an individual's use of healthcare services. ${ }^{9}$ The most recent model, the Emerging Model of Health Services Utilization (1995) includes an additional category; Outcomes which include variables that describe an individual's self perceived health status, evaluated health status and satisfaction with the health service received (Appendix A). It is suggested that these Outcome variables by way of a feedback loop play a role in whether or not an individual having accessed health services for the first time will continue to access these services in the future. The model suggests that self perceived health status, evaluated health status and satisfaction with the health services received may influence this decision. ${ }^{9}$

Andersen's models have been used extensively nationally and internationally as a framework for utilization and cost studies of general populations as well as special studies of minorities, low income groups, children, women, the elderly, oral health, the

homeless and the HIV-positive population. ${ }^{10-15}$ The current thesis uses an adapted version of Andersen's 1995 Behavioral Model of Health Services Utilization.

\subsection{Background}

In general, the development of chronic disease results from a variety of personal, environmental and social factors. These diseases include cardiovascular diseases such as hypertension, chronic respiratory diseases such as asthma and chronic obstructive pulmonary disease, diabetes, mental illness and musculoskeletal diseases such as arthritis.

Some of the main risk factors for chronic disease include smoking, sedentary lifestyle, insufficient consumption of fruits and vegetables and excess body weight. ${ }^{2}$ For 
example fruits and vegetables are important components of a healthy dict. Accumulating evidence suggests that they could help prevent major diseases such as cardiovascular diseases, and certain cancers, principally of the digestive system. Low intake of fruit and vegetables is estimated to cause about $19 \%$ of gastrointestinal cancer, $31 \%$ of ischemic heart disease and $11 \%$ of strokes worldwide. Approximately 2.7 million deatlis annually are attributable to low fruit and vegetable intake across the globe. ${ }^{10}$ Excessive overweight or obesity is another important risk factor for the development of chronic discases, namely those associated with cardiovascular diseases, diabetes and musculoskeletal diseases. Over the last 30 years, using body mass index (BMI) as a reflection of excess weight, there has been an increase in adult obesity from approximately $10 \%$ in 1975 to $20 \%-30 \%$ in 2005 in most developed countries. ${ }^{17}$ The current study will focus on whether obesity as a risk factor for the development of chronic diseases plays a role in increased utilization of health services.

The word 'obesity' is derived from the Latin 'obesus' meaning "very fat'. 18

According to the WHO, obesity is characterized as excessive body fat or adipose tissue or an increased body weight beyond skeletal and physical standards as the result of an excessive accumulation of fat in the body. More than two times the normal weight is considered to be obese. ${ }^{19}$ Obesity is a complex disorder of endocrinological, genetic and environmental aetiology. Excess adipose tissue increases the work of the heart, allers pulmonary, endocrine and immunological functions and may result directly in obstructive sleep apnea, osteoarthritis and psychosocial dysfunction. Enlarged fat cells increase the secretion of inflammatory and coagulation molecules, and may cause increased risks of 
diabetes, gallbladder discase, high blood pressure, hyperlipidemia, liver disease, coronary artery disease, cerebrovascular discase, and certain types of cancer. $2,16,17$

There are a varicty of methods for assessing an individual's body fat and these will be discussed in Chapter 3. The current study uses BMI as a reflection of excess body weight and will be discussed in the following section. BMI is the most commonly used noninvasive method for classifying body weight associated with health risk in population studies. BMI is calculated by dividing the weight of an individual in kilograms by the height of an individual in metres squared $\left(\mathrm{kg} / \mathrm{m}^{2}\right)$. BMI correlates strongly with direct measures of fatness or adiposity $(\mathrm{r}=0.82-0.91)$ in adults. ${ }^{20} \mathrm{BMI}$ is endorsed by the WHO, the International Obesity Task Force (IOTF) and Health Canada as a reflection of body fat, and has become an aceepted index for international comparisons at a population Ievel. The current recommendations for BMI elassification in Canada are the same as the standardized WHO classification of BMI: underweight $(<18.5)$, normal weight (18.524.9), overweight or preobese (25-29.9), obesity class I (30-34.9), obesity class II (35$39.9)$ and obesity class $I 11$ or morbid obesity $(\geq 40)$. For the purpose of this thesis due to the small number of individuals classified as class III or morbid obesity, obesity class II and obesity class III were combined and described as morbid obesity.

BMI ean be calculated using either self reported heights and weights or measured heights and weights. The analysis in this thesis is based exclusively on BMI calculated from self reported heights and weights. Calculating BMI using the measured height and weight approach can provide a more aceurate description of weight status in the population, however it is costly to obtain and not always feasible for population studies. 
Studies using the measured height and weight approach have been limited, due to the lack of the measured data in Canada and in many other countries. When conducting population studies, estimating BMI based on self reported heights and weights is often the only method available.

A plethora of research suggests that obesity is a health state associated with chronic diseases (e.g., cardiovascular disease, hypertension, cancer, diabetes mellitus), endocrine and metabolic disturbances (e.g., insulin resistance, dyslipidemia), debilitating health problems (c.g., osteoarthritis, gout, pulmonary diseases), psychological issues (e.g., social bias, prejudice, discrimination, psychological effects and cating disorders ${ }^{21}$ and carly death. ${ }^{22.23}$ Obesity is considered to be the second leading avoidable cause of mortality for adults in developed countries. Smoking is considered the first. ${ }^{24}$

Based on the established relationship between obesity and chronic disease it is important to study the impact of increasing levels of BMI on health services utilization to determine whether obesity is an independent predictor of utilization in the healtheare system or whether the relationship is mediated by chronic disease. Understanding this relationship may assist policy makers in making effective resource allocation decisions.

In Canada, over the past two decades, there has been a dramatic increase in the prevalence of obesity as measured by a BMI $\geq 30 \mathrm{~kg} / \mathrm{m}^{2}$. Some of the earliest data published on weight distribution in Canada comes from the Health Promotion Survey conducted in 1985. This survey was representative of the national population. Based on self reported heights and weights, the authors reported a prevalence of adult obesity in Canada of $5.6 \% .^{25}$ 
Ten years later, according to the National Population Health Survey conducted by Statistics Canada in 1994/1995, the self reported prevalence of adult obesity in Canada had increased to $12.7 \%{ }^{25}$ and the corresponding prevalence of obesity in NL increased to $15.6 \%{ }^{26}$ The 2005 Canadian Community Health Survey reported that the prevalence of self reported obesity in Canada had further increased to $16 \%$ and the corresponding prevalence of adult obesity in NL had increased to $27 \%$. Using measured heights and weights from the 2004 Canadian Community Health Survey, Statistics Canada reported $24 \%$ of the Canadian adult population and $34 \%$ of the NL adult population to be obese. ${ }^{27}$ Consequently, it would appear that NL is a particularly relevant place to study the relationship between obesity and its impact on the healtheare system.

Using Andersen`s Behavioral Model of Health Services Utilization (1995) as a guide, the relationship between increasing category of BMI as a reflection of excessive boly fit, chronic disease and health services utilization is explored. To this authors knowledge BMI as a reflection of health status has not been included in the Andersen model as a factor that influences either directly or indirectly the use of health services."

\subsection{Problen Statenent}

The level of chronic disease is increasing in most populations in the world as is the proportion of healtheare budgets spent on the treatment and management of chronic illnesses. Excessive bodyweight or obesity is one risk factor contributing to the development of chronic disease. Due to the increasing prevalenee and the large proportion of people affected, obesity has becone a public health issue. ${ }^{17,21}$ The public health coneern over the increasing prevalence of obesity is both medical in terms of 
morbidity and economic in terms of costs to the healtheare system. Limitcd research has been conducted to date in Canada on the problem of adult obesity and its impact on the healthcare system. Newfoundland and Labrador has the highest provincial prevalence of obese adults in Canada. In this province, approximately three out of every ten adults are obese (BMI $\geq 30$ ), four out of every ten are overweight $(25<\mathrm{BMI}<30)$ and three out of ten have a BMI in the normal range, which is associated with the least risk of ill health $(18.5 \leq \mathrm{BMI}<25)$. Less than $1 \%$ are considered to be underweight $(\mathrm{BMI}<18.5)$.

The main research hypothesis in this study is that increasing levels of BMI will impact the healthcare system in terms of increased utilization and direct cost. The purpose of this study was to examine and quantify the relationship between level of BMI, chronic disease and the impact on healtheare service utilization.

The study sample was representative of the adult population living in Newfoundland and Labrador in 2001. The following components were measured (i) self reported chronic disease (ii) self reported health service utilization (iii) objective health service utilization over a five-year period and (iv) an estimate of the associated direct cost to the healthcare system.

An innovative methodological approach was undertaken to study these relationships. A random representative sample from the Newfoundland and Labrador portion of the 2001 Canadian Community Health Survey was used to ascertain the study population. This sample was linked via a unique identificr, the health insurance number, (the MCP number in NL) to two longitudinal administrative databases, the Medical Care Plan [MCP] database which is the physician claims database and the Clinical Database 
Management System [CDMS] which is the hospital separation discharge database.

Through these databases, it was possible to obtain actual health service utilization and dircct costs for the study sample between 1998-2003.

\subsection{Research Questions and Objectives}

The author of this thesis studied individuals responding to the 2001 Canadian Community Health Survey. The author examined the relationships between category of BMI and chronic disease, category of BMI and health services utilization and the relationship between chronic disease and health services utilization. Andersen`s Behavioral Model of Health Services Utilization was used to guide the research.

The objectives of the study wcre:

1. to determine the factors associated with being classified as obese or morbidly obese

2. to determine whether increasing category of BMI is associated with an increase in the self reported prevalence of chronic disease

3. to determine if there are signifieant differences in self repoited health services utilization across categories of $\mathrm{BMl}$

4. to determine whether individuals with certain characteristics (e.g., demographics, health status or lifestyle variables) are more likely to be either obese or morbidly obese

5. to determine if individuals reporting the existence of chronic diseases are more likely to be classified as overweight, obese or morbidly obese 
6. to determine if there are significant differences between self reported and objective measures of physician utilization

7. to determine if there are significant increases in the use of objective health services and associated direct costs with increasing eategory of BMI (the shape of the relationship is not known)

8. to evaluate using multivariate models the relative importance of increasing category of BMI and the level of chronic eomorbidity on health services utilization and direct costs

9. to examine the relationships between obesity, chronic disease and health services utilization using the Andersen model of Health Services.

\subsection{Rationale}

Provincial governments in Canada spend a large proportion of their budgets on the healtheare system. Aceording to data published by $\mathrm{ClHI}$, this proportion varies across Canada from a low of $7.2 \%$ of GDP in the North West Territories to a high of $13.3 \%$ of GDP in Prince Edward Island. Newfoundland and Labrador spent $10.9^{\circ} \%$ of its GDP on healtheare in $2004 .{ }^{28}$ With the majority of funds being directed towards medications, hospital and physician services it is becoming increasingly important for policy makers to not only have information on health services utilization but to also understand what factors drive utilization. Due to the considerable evidence that overweight and obesity are related to the increased risk of chronic discasc, it is suggested that being overweight and or obese may be a large contributor to increased utilization of health services and therefore rising healtheare costs in many countries. 
As a result, studies have been conducted to explore the relationship between (i) the level of BMI and health services utilization ${ }^{29-38}$ and (ii) the economic burden placed on healthcare systems associated with different levels of BMI. ${ }^{39-57}$ These relationships have not been studied extensively in Canada which is surprising given the reported increase in the prevalence of obesity since the $1980^{\circ} \mathrm{s}$.

In one of the early studies conducted in Canada, researchers performed a secondary analysis of the 1994 National Population Health Survey which was a crosssectional survey representative of the Canadian population. Using multivariate logistic regression models researchers examined the relationship between obesity and the use of health services, including medications, comparing it to the non-obese population. The authors controlled for age, sex, marital status, income, level of physical activity and smoking status in the models. The findings, based on self reported health services utilization, and using the previous Health Canada guidelines (which changed in 2003) for classifying obesity (BMI $\geq 27 \mathrm{~kg} / \mathrm{m}^{2}$ ), indicated that being obese was associated with increased physician visits. Furthermore, obese respondents when compared to non-obese respondents were more likely to have reported suffering from a number of chronic comorbidities (e.g., diabetes and cardiovaseular disorders), to have been preseribed a larger number of medications (c.g., heart medication, antihypertensive pills, diuretics, pain relievers for arthritis and back pain) and to have sought out mental health services. In contrast, obese respondents had fewer hospital admissions than non-obese respondents. ${ }^{31}$ 
In 2005, the Canadian Institute for Health Information (CIHI), an independent pan-Canadian, not-for-profit organization published a report based on the Canadian Joint Replacement Registry. BMI values were available for over 17,000 patients, which accounted for $65 \%$ of hip and knee replacements reported in the study. Adjusting for age and gender, people who were obese $\left(\mathrm{BMI} \geq 30 \mathrm{~kg} / \mathrm{m}^{2}\right)$ were three times more likely to have received a hip or knee joint replacement, compared to people with normal weight. Overweight individuals (BMI 25-29.9 kg/ $\mathrm{m}^{2}$ ) were twice as likely to have needed a joint replacement. These findings add support to the link between obesity as a risk factor for the development of osteoarthritis, a key diagnosis leading to joint replacement surgery. In addition, the authors reported that being obese increased the necessity for repeat surgeries or modifications or replacements of the artificial joints, because the extra weight added more wear and tear on the individual's prosthesis. ${ }^{37}$

It appears that during a time when obesity is increasing in the population, the number of joint replacements has also been increasing suggesting a positive alssociation between the two. The relationship may be eausal; either the hip or knee problems have caused decreased mobility resulting in increased weight gain, or the weight gain may have caused the knee and hip problems and subsecpuently, the number of joint replacements being performed.

A number of studies have been conducted outside Canada, exploring the relationship between obesity and healtheare utilization. Authors conducted a secondary analysis of the Health Survey for England, a cross-sectional survey designed to collect information about the health of the English population. ${ }^{35}$ The relationship between 
obesity and the use of healtheare services in a population 50 to 69 years of age was examined. The authors reported an association between obesity $\left(\mathrm{BMI} \geq 30 \mathrm{~kg} / \mathrm{m}^{2}\right)$ and the increased use of outpatient services such as surgical daycare over the previous 12-month period compared to the normal weight group $(\mathrm{BMl}>18.5$ and $<25)$, having controlled for age, gender, smoking status, alcohol intake, socioeconomic status, ethnic groups and education groups. There were no significant relationships reported between obesity and physician visits in the pervious 2 weeks or between obesity and the use of inpatient services in the previous 12 months. The authors did not control for the existence of chronic diseases and did not examine different levels of obesity. It may be that a significant relationship between BMI and increased utilization of physician and or hospital services did exist, but only with those individuals classified as being morbidly obese that is with a $\mathrm{BMl} \geq 35 \mathrm{~kg} / \mathrm{m}^{2}$.

In a study in the USA, researehers randomly allocated 506 new patients to resident family physicians and followed their health serviee utilization patterns for a period of 12 consecutive months. ${ }^{38}$ Analyzing the findings by BMI classification using measured heights and weights, the authors found that obese patients (BMI $>30 \mathrm{~kg} / \mathrm{m}^{2}$ ) compared to non obese patients (BMI $<30 \mathrm{~kg} / \mathrm{m}^{2}$ ) had a significantly higher mean number of visits to both primary care and specialty care clinics, and had a higher mean number of diagnostic services. Controlling for health status, depression, age, education, income and sex, obesity remained significantly associated with the use of primary care services and diagnostic services. When the level of morbidity was controlled for in the analysis, obesity was associated with primary care visits only. 
A further study limited to women investigated health services utilization in the previous 12 months in a primary care setting. The authors reported that being obese $(\mathrm{n}=83)$ with a $\mathrm{BMI} \geq 30 \mathrm{~kg} / \mathrm{m}^{2}$, compared to being non-obese $(n=111)$ with a $\mathrm{BMI} \cdot 30$, was associated with a greater number of diagnoses, contacts with the medical facility (including telephone contact), the total number of prescriptions ordered and number of physicians seen. ${ }^{33}$ Having eontrolled for the number of diagnoses, BMI remained a predictor of the number of physicians seen suggesting that the higher utilization of obese patients is due to the higher level of morbidity.

In a study examining more than 17,000 members of a large health maintenance organization (HMO) in the United States, secondary analysis was conducted on computerized databases that included information on hospitalizations, laboratory services, outpatient visits, outpatient pharmacy and radiology serviees during 1993. The authors reported that an increase in BMI was associated with more frequent outpatient visits as well as increased inpatient days, which was most likely due to obesity-related comorbidities such as hypertension, coronary heart discase and diabetes. ${ }^{4}$

Associated healtheare costs for obesity related comorbidities have been estimated to be between $2-7 \%$ of total healtheare spending in most industrialized countries. ${ }^{36-54,56-57}$ In Canada in 1999, it was estimated that the total direet healtheare cost of obesity was over $\$ 1.8$ billion annually, or approximately $2.4 \%$ of the total healtheare expenditures for all discases in Canada. ${ }^{\text {to }}$

The findings from many of these studies are consistent at the macro level; obese individuals $\left(\mathrm{BMI} \geq 30 \mathrm{~kg} / \mathrm{m}^{2}\right)$ use more of "some" types of healtheare services when 
compared to non-obese or normal weight individuals. However, there are inconsistencies in a number of the study findings. While some authors reported increased utilization of primary care services, specialty clinics, hospital outpatient and inpatient visits and number of diagnostic tests, other authors reported increases in only some of these services by obese populations. These inconsistencies may be due to the differences in study methodology. These included the choice of study population (i.e., patients versus population survey respondents and age-groups studied) as well as the outcome variables being examined (i.e., self reported versus actual health services utilization). In addition the study results may be inconsistent depending on whether BMI was calculated using self reported or measured heights and weights. This difference in methodology may lead to the misclassification of BMI status. For example overweight and obese individuals are more likely to underestimate their weights resulting in an underestimation of BMI. Often this misclassification will result in fewer differences being found between the groups. Finally, a difference in the time period of follow-up and the choice of statistical analysis, including decisions to adjust for confounding variables may have impacted the study results.

This thesis addresses a number of methodological limitations raised in other studies. First, despite the fact that several studies have been conducted in this area of research very few studies have been Canadian in nature. Different healthcare systems, varying degrees of access to care, population demographics such as the changing age structure and geography (e.g., rural versus urban) as well as cultural issues and illness behavior are unique to different populations. These factors may limit the generalizability 
of prior study findings to Canadian populations. Second, although other studies may have used national population health surveys to ascertain a sample population, very few have focused on a particular provincial population. This is important because as the prevalence of overweight and obesity varies across the eountry, so does the likelihood of chronic diseases and the potential reasons for healtheare services utilization. Among Canadians, the prevalenee of overweight and obesity is the highest in NL. Using National data to draw conclusions about specific provinces may not be valid. To overcome this issue, this thesis used the NL component of the 2001 Canadian Community Health Survey; a population based cross-sectional survey of randomly selected respondents. Third, previous studies have not consistently controlled for factors known to have an impact on health services utilization. For example, in a study in the United States described earlier where 506 new patients were randomly allocated to family physicians and followed for a period of 12 consecutive months, the authors did not adjust for obesity associated comorbid conditions such as hypertension and type II diabetes in their analysis. The authors argued that statistically adjusting for comorbidities was inappropriate as many of these conditions are intermediates along the causal pathway between increased BMI and increased health services use and therefore constitute over adjustment. ${ }^{38}$ The author of this thesis agrees that chronic conditions may be the link between obesity and increased health services utilization, but suggests that it may be valuable in terms of policy development to better understand if obesity on its own is independently predictive of health services utilization, or whether it is primarily the relationship as a risk factor with 
chronic disease that is driving healthcare utilization and the increased cost of healthcare services.

Fourth, the majority of previous studies have relied solely on self reported health services utilization data obtained from health surveys, with varying time periods for the purposes of recall. Although, this study utilized health survey data to obtain the study population, self reported chronic disease and health services utilization, as well as other variables important to the study; it also linked each survey respondent via a unique identifier to actual health services utilization collected over a 5-year period.

The final methodological limitation raised is that the majority of studies published on the cost of obesity to the healthcare system have used a top-down prevalence-based approach. While population-based data are valuable, it is also important and useful to quantify the association of obesity and healthcare resource cost at the patient or individual level. Linkage at the individual-level to healthcare utilization and subsequent costs allows for examination of the relationship between obesity and health services utilization while controlling for known confounders such as patient health status, socioeconomic status, age and gender. This study utilized individual-level data, controlling for factors known to have an impact on health services utilization and estimates the cost of actual physician and hospital services using the physicians' claims database and a hospital costing methodology developed by CIHI.

Finally, this thesis uses Andersen's Behavioral Model of Health Services Utilization (1995) as a guide in the design and the analysis of the study. The author attempts to understand the relationship between category of BMI and its impact on health 
services utilization as a Health Behavior. As well, the study explores the association between category of BMI and chronic disease, and whether BMI on its own is identified as a predisposing or need variable in terms of the model.

\subsection{Thesis Outline}

Chapter 1 presented an introduction to the research, a rationale for the study, the research hypothesis and the specific research questions. Chapter 2 provides an introduction to the theoretical model used in the thesis and a literature review of the relationship between obesity and health services utilization. Chapter 3 provides the context for a study in obesity including a review of obesity related topics, the measures of obesity and its association with the burden of illness and its epidemiology. Chapter 4 presents a discussion of the methods used in this study including the use of individuallevel data, details of data sources, the data linkage process and the data analysis. Chapter 5 presents the results from the analyses. Chapter 6 provides a discussion of the findings, the conclusions and policy implications as well as the study limitations and potential arcas for future rescarch. 


\section{CHAPTER 2 Theoretical Model and Literature Review}

\subsection{Healthcare Utilization}

Health services are part of the largest sector of the economy and these services continue to grow. The provision of health services is purported to maintain and improve the health status of the population.

Utilization of healtheare services can be defined by purpose: primary care has to do with preventive measures of illness; secondary care refers to the process of treatment in an attempt to return an individual to a previous state of functioning while tertiary care describes services for acute care health problems such as a ruptured aneurysm or myocardial infarction as well as for the stabilization of long-tern irreversible illness such as heart disease or diabetes. These services are often provided in large, well staffed, cquipped facilitics. ${ }^{7}$

A number of approaches have been developed to help explain an individual's decision to access and use healtheare services. The socio-demographic approach suggests that variations in healthcare utilization are related to variables such as age, sex, education, occupation, ethnicity, socioeconomic status, income as well as the structure of the health system and the external environment. The social-psychological approach sets out to explain factors that influence the secking of care, suggesting that three main factors influence a patient's decision to seek help: knowledge, beliefs and attitudes concerning symptoms; attitudes and expectations regarding physicians and health services in general and a definition of sickness and determination of the necessity for professional care. The organizational approach examines the structure of the healtheare delivery system in order 
to account for differential health and illness behavior. For example whether the healthcare system is privately or publicly funded will impact the provision of healthcare services and who has access to them. Finally, the social systems approach views the healthcare system as consisting of interrelated components such as physicians, facilities and populations within the healthcare system. This approach suggests that these components interact with one another and the population they serve. It postulates that there is an interconnected relationship between the social structure of the population, the health services provided and the health status of the population and that these factors in turn impact healthcare utilization. ${ }^{7}$

Each of these approaches provide insight into the type of factors that influence the utilization of healthcare services and several models have been developed that have included and integrated different components of each approach in an attempt to understand what specific factors influence an individuals use of healthcare services. One such model is the Behavioral Model of Health Services Utilization developed by Andersen in 1968 as part of his doctoral dissertation. Andersen viewed the use of healthcare services as another form of human behavior or human activity. One of the earlier adaptations of this model by Andersen \& Newman (1973) integrated variables that might be found in the socio demographic and the organizational approaches. The latest model published in 1995 integrates many of the factors included in the Social Systems Approach and takes a much broader view of factors impacting health behavior, recognizing the complexity of health services utilization. The Andersen \& Newman Model has often been used to guide research and evaluation studies on healthcare access 
and utilization, ${ }^{10-15}$ and has been used to explain the utilization of physician services, ambulatory care, psychiatric care, emergency room care, dental care as well as preventive services. ${ }^{8-15}$ This model has been applied to specific groups such as veterans, the urban poor, the homeless, Hispanic immigrants and the elderly. ${ }^{58-02}$ As well, in Canada, this model has been used to compare the use of emergency room services and services provided by allied health professionals. ${ }^{63-64}$

\subsection{Andersen's Behavioral Model of Health Services Utilization}

Andersen`s Behavioral Model of I lealth Serviecs Utilization (1995) is a complex model. The main purpose of the model is to aid in understanding variables impacting healtheare utilization in either a positive or negative manner (Appendix $A$ )." The model suggests that Health Outcomes defined as perecived and evaluated health status, as well as consumer satisfaction are the result of the interaction of Environmental factors, Population Characteristics and Health Behaviors. A description of the model follows. For the purpose of the current thesis the outcome of interest is Health Bchavior not Health Outcomes.

\subsubsection{Environmental Factors}

Envirommental factors describe the context in which utilization takes place and can be separated at an aggregate level into two main headings: the health system and the external environment. Health system variables refer to policies, provider organizations and the methods of finanee. The external environment includes: the economy at the time including the societal norms and structures found in the population. At an individual level, environmental factors include measures of the community and the provider. 
Community variables relate to the geographical locations such as where individuals live and where they receive healthcare services (e.g., rural versus urban residence). Provider characteristics relate to the way in which services are provided with a focus on payment systems. Variables describing the Environment were not directly examined in this study as there was limited information in the health survey available in this context.

\subsubsection{Population Characteristics}

Population Characteristics describe three groups of variables classified under three main headings: predisposing, enabling and need. The variables found under these headings have been found to demonstrate an impact the use of health services.

\subsubsection{Predisposing Factors}

Predisposing factors refer to individual characteristics which create a greater propensity in some individuals to use more services than others. ${ }^{8}$ These characteristics are not directly responsible for health service use but predict either directly or indirectly utilization. The 1995 model describes five groups of Predisposing variables:

demographic, social structure, health beliefs, psycho-social attitudes and genetic traits."

Demographic factors include variables such as age and gender. These variables are related to the use of healtheare services." For example, age has been found to be a significant predictor of health services utilization; as age increases so does the likelihood of ill-health. Gender is also associated with specific utilization patterns. For example women are much more likely to avail of health services during their reproductive years and through menopause. 65 
Social structure describes the living conditions of an individual and provides some context for their individual choices. Social structure variables consist of variables such as marital status, family size, residential mobility and occupation and tend to have a small impact on overall health services utilization."

Health related beliefs describe individual attitudes toward healtheare services, physicians and disease. They also reflect individual knowledge about illness and the healthcare system. It is suggested that these factors are important predictors of health behavior, for example what an individual thinks about health may ultimately influence health and illness behavior. In addition an individual's psycho-social attributes such as problem-solving and coping skills are also recognized as significant predisposing variables. ${ }^{9,66-67}$ More recently, with the advances in the diagnosis and the treatment of genetic diseases, Andersen has recognized the significant role that genetics may have on health and subsequent service utilization and has suggested that it be included as a Predisposing variable in the model. ${ }^{9}$ According to Andersen and others, Predisposing factors are not directly linked to utilization, but are related indirectly through their relationship with the other Population and Need characteristics. 8.9

\subsubsection{Enabling Factors}

Enabling factors refer to those variables that represent an individual's ability to aceess healtheare services such as income level, health insurance and regional access. For example, in the United States individuals must have some form of insurance or pay outof-pocket for health services used. 
In Canada, Medicare, the national healtheare system, ensures universal healtheare coverage for all residents of Canada that is free at the point of entry for physician and hospital services and as a result it is unlikely that enabling variables play a large role within Canada in an individual's ability to access these particular services.

\subsubsection{Need Factors}

Provided that predisposing and enabling conditions exist, the individual must have a need in order for the use of health services to take place. Necd variables include

health status or measures of illness ${ }^{8,9}$ and they are often described as the most important

cause of healthcare utilization. ${ }^{9,58,60)}$ Need can be measured in a varicty of ways including: measures of subjective health, restricted activity days or disability days, the presence of chronic disease, the health utility index (HUI) which is a derived variable that measures several indicators of physical health such as vision, mobility and hearing as well as health status. Need factors have been shown to be associated with the use of health services."." In the current thesis, Need is operationalized by the total number of self reported chronic conditions that an individual reports having being diagnosed with by a health professional as well several indicators of health status.

\subsubsection{Health Behavior - Outcome of Interest}

In Andersen`s Behavioral Model of Health Services Utilization, the eomponent described as health behavior refers to the type and volume of healthcare service utilization such as the utilization of services provided by physicians. In the current thesis, two outcomes of interest were described as Health Behavior. These included: (1) self 
reported utilization of physician and hospital services (2) objective utilization of physician and hospital services and associated costs.

\subsubsection{Outcomes}

More recently, in Andersen's model (1995), a final category was added describing Outcomes associated with healthcare use. These Outcomes include variables describing perceived health status, evaluated health status and consumer satisfaction. Perceived health status is the individual's impression of the severity of the health issue incorporating an individual's health beliefs, values and attitudes, ${ }^{8,9}$ and may change based on changing level of education, illness behavior and expectations of the health

system. Evaluated health needs describe the health professional's clinical diagnosis and expert advice on what is needed in the provision of medical services. For example whether further follow-up, treatment or diagnostic testing is required." Consumer satisfaction refers to how satisficd a patient or client is with the health scrvice provided by a health professional and is unique to each individual based on their own set of experiences and expectations as well as the effectiveness of the service provided. In the current thesis the Outcomes described in Andersen`s model was not evaluated. 


\subsection{Obesity and Health Services Utilization}

The prevalence of overweight and obesity is increasing in most developed countries and its association as a risk factor for the development of chronic discases has prompted researchers to examine its impact on the healtheare system in terms of healthcare utilization and costs.

According to the latest OECD data (2006), the United States when compared to other developed countries reports the highest prevalence of overweight and obesity, and therefore it is not surprising that many of the studies assessing the relationship between obesity and its impact on the healtheare system have been published using American data. ${ }^{24,30,34,36,38,41,44-45,68-619}$ Given the differences in health systems between the United States and Canada, it is not entirely clear whether the study results are generalizable to the Canadian population. In Canada, under a national system of Medicare all residents in provinces and territories have universal aceess to both physician and hospital services free at the point of entry. In the United States, aceess is limited to those individuals covered by some form of health insurance with a bias towards those individuals who are employed. As well, several studies assessing the relationship between obesity and its impact on the healtheare system have also been conducted in European countries where the health systems are a mix of private and public provision. $33,35,70-76$

There is a dearth of literature in this area to draw upon in Canada. ${ }^{31,46-47,77}$ and although the Canadian system is similar in some ways to European healtheare systems in its method of public finance, provision and universal coverage, it is not known whether the results of these foreign studies are truly generalizable to the Canadian population 
given the difference in culture, illness behaviour, ethnicity and access. These studies have been conducted in the context of different countries, with different healtheare systems, on a variety of populations with varying age-ranges, different timelines and various methodologies. Therefore there is a need to conduct regional studies in Canada to examine this relationship to ensure its gencralizability to the population and to provide robust conclusions for the development of policy.

\subsubsection{Obesity and Visits to a Primary Care Provider}

A number of studies conducted in several countries have reported a significant relationship between obesity and the increased use of ambulatory care services and visits to a general practitioner (GP). ${ }^{31,34,38,41,44,69,71,73}$ These have included studies that stratified their study populations by gender, ${ }^{32,45.70}$ as well as those studies whose focus was women only. ${ }^{33,72}$ Many of these studies found a significant relationship between obesity and increased utilization of GP services, however these particular studies did not control for the presence of chronic diseases. $31,38,71$ It may be that it is the presence of ehronic discase and not obesity per se that demonstrates the direct effect on the need for healtheare services and although obesity is a risk factor for the development of chronic disease its impact maly be indirect.

A secondary analysis of the cross-sectional component of the 1994 National Population Health Survey data was conducted. This longitudinal survey was representative of the Canadian population. Based on self reported information, the findings indicated that being obese (BMI > 27) was associated with increased visits to a GP. The following variables were entrolled for in the regression analysis; age, sex, 
marital status, income, level of physical activity and smoking status. The existence of chronic disease was not controlled for. The prevalence of chronic co morbidities such as diabetes and cardiovascular disorders was higher in the obese population compared to the non obese population. ${ }^{31}$

In the study described earlier in Chapter 1, 506 new patients (either obese or nonobese) at the Davis Medical Centre Primary Care Center, University of California were randomly assigned to 105 Primary Care resident physicians and their subsequent healthcare utilization patterns were compared. ${ }^{38}$ Information was collected on socio demographies and the heights and weights were measured. As well other information was collected on the use of medical services during a prospective $12-$ month period and was determined by a review of medical records. The authors reported that obese patients had a higher mean number of visits to both primary care and specialty eare clinics, and a higher mean number of diagnostic services ordered compared to non-obese individuals (BMI $<30)$. The authors reported that being obese remained significantly related to the use of primary care and diagnostic services after controlling for health status, depression, age, education, income and sex. The authors did not adjust for obesity associated comorbid conditions such as hypertension or type II diabetes. They argued that statistically adjusting for comorbidities was inappropriate as many of these conditions are intermediates along the causal pathway between increased BMI and increased health services use and therefore including them in the statistical model would constitute over adjustment. The authors included a surrogate for comorbid conditions, al variable that measures physical health (MOS SF-36) and one that measures the impact of 
comorbidities on self-reported health. Although this study was a prospective study design in contrast to many of the other studies reviewed, there were several limitations. It was conducted at a university medical centre with primary care residents, therefore the care provided and the population serviced may be somewhat different from what is found in the community. In addition due to the prospective nature of the study the resident physicians were not blinded. It is possible that some bias existed in that resident doetors knowing the documented adverse affects of excess body weight may have unconsciously seen their obese patients more often and ordered more diagnostic tests for conditions such as hypertension, high cholesterol and type 2 diabetes.

In a study on the German population, the authors analyzed responses to the KORA-survey 1999/2001, a health survey administered to the adult population 25 to 74 years of age in the Augsburg region. The study included 947 respondents. The number of visits to a GP was analyzed and BMI was based on measured heights and weights. Having controlled for sex, age, place of residence, social class and sickness fund which is a type of health insuranee fund for those individuals employed (these variables were described as predisposing and enabling variables as per Andersen's model), obese individuals with a $\mathrm{BMI} \geq 35$ were associated with more frequent utilization of GP services compared to normal weight individuals. Chronic diseases were not adjusted for in this analysis. ${ }^{71}$

Studies stratified by gender found similar results; in general, obesity was assoeiated with increased ambulatory care, including visits to a GP, although the results were not always consistent between genders. ${ }^{2,45,70}$ An Australian National Health 
Survey was conducted in 1995. Survey responses were available for 17,033 men and 17,174 women older than 20 years of age on health-related issues. Secondary analysis of this data was performed. Having controlled for age and income, a positive relationship was reported between BMI based on self reported heights and weights and some measures of healtheare service use for both men and women. Obese men and women were more likely to have made a visit to a primary care doctor and obese women only were more likely to have made a visit to a specialist. Both obese men and women were more likely to have reported visiting an outpatient clinic in the two weeks before the interview. ${ }^{32}$

Data from the Health and Retirement Study was examined in order to estimate the effect of weight class on healtheare use. This study was a nationwide biennial longitudinal survey representative of American adults between 50 and 69 years of age. Analysis of the data demonstrated a monotonic relationship between weight class measured by BMI, based on self reported heights and weights (overweight, moderate obesity and severe obesity) and an increase in outpatient healtheare serviecs for women only. Men classified with severe obesity were associated with increased outpatient visits only. No specific services were mentioned. The authors controlled for socio demographic factors (e.g., age, race, insurance status, marital status, education, family income, region) and health risk behaviours (e.g., current tobaceo smoking and heavy alcohol drinking). The authors stated that the intention was to evaluate the effects of varying degrees of obesity on healtheare use and because increased risk of many chronic discases are related 
to obesity, other health conditions or level of general health were not considered as factors in these models. ${ }^{45}$

Authors examined data from a prospective cohort study (2001-2003) of noninstitutionalized individuals $\geq 60$ years of age and older which were representative of the Spanish population. Having controlled for age, education, place of residence, tobaceo use, alcohol consumption and presence of chronic disease, the authors found that obesity based on self reported heights and weights was associated with a greater number of visits to a primary care physician, for women only. ${ }^{71}$ Other studies considering women found similar results. ${ }^{33,72}$

\subsubsection{Obesity and Visits to a Specialist}

There were very few studies that examined the relationship between obesity and its impact on specialist services. For those studies referring to outpatient serviees, the services provided by a specialist were not described. In a review of the studies published the relationship between obesity and specialist services was found to be inconsistent. $^{38,70,74}$

\subsubsection{Obesity and Hospitalization}

The relationship between increasing BMI and increased utilization of hospital services such as inpatient admissions, length of stay and resources used while in hospital is found to be inconsistent. Although many authors reported a statistically insignificant relationship between obesity and increased hospitalization, $31,33,34,35,38,45,71,72$ other authors reported a significant finding that obesity increased the use of hospital

services. $^{41,4+71,75-77}$ 
One explanation for the inconsistent findings of many of these studies is that hospital inpatient admissions may not be correlated with discase prevalence. ${ }^{78}$ Hospital admissions may be more reflective of the level of serverity (e.g., more acute) and levels of aceess. This may be a limitation associated with hospitalization studies of this nature as rates of hospitalization may not be good indicators of the general level of morbidity in a population but indicative of other determinants.

Having reviewed the studies, it appears that the positive studies were similar in that they had large sample sizes and primarily examined an elderly population over long periods of follow-up. For example in a more recent study in 2006 , the authors had conducted a prospective cohort study of approximately $15,(1)(0)$ men and women who were in the age range of 45-64 years old in 1972. These survey respondents hat taken part in the Rentrew/Paisley study in Seotland. Follow-up continued until 20(0). The authors concluded that those who were overweight and obese at baseline had higher than expected rates for hospital admission and women who were overweight and obese at baseline demonstrated a u-shaped relationship between BMI and hospital admission rates. $^{75}$

The healtheare utilization patterns of the members of the Kaiser l'ermanente of Colorado, America's largest HMO were examined. This HMO has an average of 8.4 million members enrolled. Using a retrospective matched study design, obese individuals were matched with non-obese individuals by age, sex, outpatient medical office and the absence of selected diagnosis such as pregnancy, coronary artery discase, congestive heart failure and cardiac arrhythmia. The healtheare utilization was 
determined for these individuals for the previous 12 months. The authors found that obese individuals had more hospitalizations, prescriptions drugs, professional claims and outpatient visits when compared to those who were considered normal weight. ${ }^{44}$

A study on the First National Health and Examination Survey was conducted in the USA between 1971 and 1975 on adults 25 years or older. Follow-Up Surveys took place between 1982 and $1992(n=14,407)$. The authors looked at BMI classification and length of stay in hospital. The authors reported the following results. Individuals with a BMI $\geq 35$ (moderate obesity), a BMI between 30 and 34.9 (obese), and a BMI between 25 and 29 (overweight) had inpatient crude length-of-stay (LOS) rates greater than those individuals classitied as normal weight (BMI 18.5-24.9). The authors concluded obese individuals experieneed longer hospital stays when compared to those with a normal weight. ${ }^{36}$

The Medicare Beneficiary Longitudinal Survey examined the healtheare utilization of an elderly population $(n=8,754)$ between 65 and 100 years of age. This study was limited to hospitalization. The authors reported individuals in the lowest BMI (quintile had a higher risk of hospitalization than those in the middle BMI quintile.

Underweight, overweight, mild obesity and moderate to severe obesity were related to a higher risk of hospitalization when compared to normal weight individuals between 0.5 and 75 years of age. For inclividuals older than 75 years of age, underweight, overweight and mild obesity were not related to a higher risk of hospitalizations. ${ }^{34}$ 


\subsubsection{Obesity and Medication Use}

Medications constitute a large proportion of the direct healtheare costs to any healtheare system. ${ }^{3}$ As stated earlier in Chapter 1, medications account for $10.4 \%$ of total healtheare spending in Canada. ${ }^{3}$ Therefore, it is of interest to researchers, policy makers and hospital administrators, especially those funded by global budgets, to better understand the allocation of funds being spent on medications. Given the relationship between excessive overweight and the increased risk of chronic disease, one might expect to see more medications being taken by this population. Many of the studies on obesity and health services utilization examined whether excessive overweight and its association with chronic disease influenced the use of medications. In a review of several studies, the results demonstrated that an increasing number of medications were taken by the excessively overweight population when compared to the nomal weight population and these medications were often associated with the existence of chronic diseases such as hypertension or high cholesterol. ${ }^{24,31,32,33,35,41,44,73-74}$

The current thesis was not able to collect information on medication use, as there were no questions on medication use included in the health survey. As medications are a large proportion of direct costs to the healtheare system, this was felt to be an unavoidable limitation of the study. In NL, a pharmacy network is being developed that will allow this type of analysis in the future, however it will be a prospective clatabase and therefore retrospective studies before the date of initiation will not be possible.

There is some consistency in the studies reviewed; that is increasing BMI appears to be associated with an increased use of GP serviecs, some outpatient services, the 
consumption of medications and to a lesser extent hospital serviees. However there are several limitations and or biases inherent in this body of literature. Very few studies have been conducted in Canada or on a specific provincial population. Many of the studies reviewed were American or European in nature. Given the differences in the prevalenee of obcsity, chronic disease and the health systems across countries, the findings from these studies may not be generalizable to Canadian populations. Many of the studies reviewed dicl not control for factors known to have an impact on health services utilization such as age and sex, smoking, education level. In the current study a number of models were developed to examine the specific impact of BMI and several covariates on its own on health services utilization and BMI and chronic discase on health services utilization. In addition many of the studies reviewed relied solely on self reported health serviees utilization data obtained from health surveys, over varying time periods. The current study used both self reported data collected from a health survey and its linkage to health administrative databases to obtain actual information on health services utilization over a five-year period.

\subsection{Obesity and the Behavioral Model of Health Services Utilization}

According to Andersen's model, as discussed in detail in Chapter 2 Section 2.2, a multitude of factors impact the utilization of healtheare services. As a brief summary the model is eategorized into four main umbrella headings: the Environment, Population Characteristics, Health Behavior and Outcomes.

The literature review for the current thesis focused primarily on the relationship between obesity and its impact on the utilization of health services as a Health Behavior. 
Very few of the studies reviewed used a model as a guide in their examination of the relationship between obesity and health services utilization, ${ }^{69}$ although a few papers did refer to variables as predisposing or enabling factors without any further explanation. However, variables found in the Andersen model were often controlled for at the analysis stage of the studies reviewed as many of these variables such as age, gender, level of education and ineome and smoking behavior are known to have an impact on health services utilization.

Studies controlling for socio-demographic, lifestyle, and socioeconomic factors (described as predisposing and enabling factors in Andersen`s model) apart from chronic disease tended to find a positive relationship between obesity and increased use of healtheare services. $23,31,32,35,45$ Other studies found an inconsistent relationship between obesity and increased use of healtheare services. In one study the authors concluded that obesity class I and II were associated with increased inpatient, outpatient and total costs when age and sex were controlled for. When comorbidities were added to the analysis (e.g., diabetes and hypertension), the impact of obesity class I and II on increased use of healtheare services was eliminated. There remained a significant relationship between the presence of comorbidities and increased utilization. ${ }^{+1}$ In contrast, several studies concluded that obesity remained an independent predietor of health services utilization, controlling for chronic comorbidity. The authors suggested one reason for this independent relationship may be that physicians view obese individuals as more likely to develop comorbidities and therefore set up a different physician visit regime (i.c., more regular appointments). Alternatively, other researchers argue that obese individuals often 
report lower scores on indexes that measure health status and self perecived health, ${ }^{31,38}$ and may perceive themselves as less healthy and therefore visit the physician more frequently. ${ }^{33,44}$

In another study in 2002, the authors reported that Spanish overweight and obese women had increased healtheare service use; visited the physician, used hospital emergency services and took more medication compared to the normal weight group. These relationships did not change having controlled for age, education and the presence of chronic disease. The sample in this study were more educated, had a higher social elass and were younger then non responders, potentially biasing the results as individuals with higher levels of socioeconomic status may aceess preventive services more in an effort to remain healthy. As well the treatment of obesity itself may lead to greater utilization as this study reported that obese women reported worse subjective health compared to non-obese women even without chronic discase. ${ }^{3.3}$ In addition, in a study in 2004, the authors reported that having controlled for age, sex and chronic discase score, BMI remained a significant predictor of total healtheare costs, but only marginally. For each increase in chronic disease score the costs increased by $52.9 \%$ while for each increase in BMI, costs increased by $2.3 \%$. Age contributed only $1.3 \%$ increase in costs for each year. ${ }^{44}$

Some authors argued that excess overweight is a risk factor for the development of chronic disease and controlling for the level of comoridity constitutes over adjustment. For example in a study in 2002, the authors found a relationship between obesity and increased likelihood of hospitalization. The authors did not adjust for specific discases 
associated with what they termed modifiable risk factors (i.e., overweight), as these are described as "intervening variables", that is those between the risk factors and ill- health outcomes such as hospitalization, mortality and disability. ${ }^{69}$

Based on this review of the available literature, it is not clear whether obesity is associated with increased healthcare utilization either directly or indirectly through the existence of chronic disease. Some of the studies reviewed took into account what might be considered to be predisposing variables such as age, sex, education and income, as well as region of residence considered to be enabling factors in the Andersen model. Some studies took level of comorbidity or chronic disease into account (the need variables) while others argued that obesity is associated with chronic discase and therefore controlling for these diseases amounts to over adjustment.

\subsection{Cost of Obesity}

Over the last decade researchers have provided many estimates of the cost of overweight and obesity to the healtheare system. Numerous studies have been undertaken in order to estimate the costs associated with obesity. The majority of these studies have

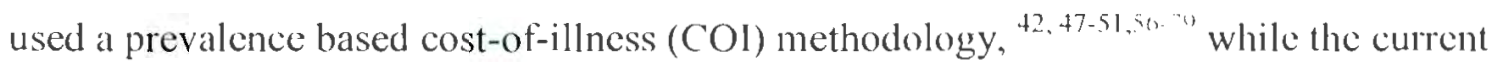
study and others have used individual-level data. $40-41,45$

Cost-of- illness studies are a type of economic analysis often used in health research. The aim is to identify and measure all costs attributable to a discase which include direct health care costs and indirect costs such as losses in productivity. Using these estimates, the overall impact is presented in monetary terms. This analysis provides health policy makers and decision-makers with information on which to make decisions 
on resource allocation. There are two types of Cost-of- Illness studies; the prevalencebased and the incidence-based approach. The prevalence-based approach is the most common approach used to study the impact of obesity on healtheare costs from the perspective of the healtheare system. This approach identifies the costs incurred during a specific time period by individuals with a particular disease. ${ }^{48}$ It is difficult to quantify the long-term consequences of a chronic disease such as obesity using the prevalencebased approach, as the information used in this type of analysis is most often based on information collected using cross-sectional study designs. The incidence-based approach follows newly classified cases of obese individuals and is more appropriate for studying the impact of obesity on healtheare costs. This approach as well estimates the lifetime cost of new eases of obesity diagnosed in a given year. However, this approach is more difficult to apply and it is very data intensive. Individuals are selected when first diagnosed or elassified with obesity, and these individuals are followed for many years to determine the development of associated eo morbidities.

\subsubsection{Prevalence-Based Approach}

The prevalence-based approach uses a top-down method calculating the Population Attributable Risk Fraction (PAF). This approach uses the relative risk associated with obesity and the development of associated chronic diseases as well as using the prevalence of obesity in the relevant population. The PAF determines the pereentage of disease prevalence due to obesity and estimates the total costs of obesity using published data on national healtheare expenditures associated with particular diseases. The calculated proportion of eases and costs of a given disease attributable to 
excess weight is greatly intluenced by the accuracy of epidemiologic evidence about disease prevalence and relative risk. It is also influenced by the presence of confounding factors affecting risk such as age, sex, race and the presence of co-morbid conditions. In addition, factors determined by the investigator such as the BMI, affect the relative and attributable risk estimates and are often different in different populations.

Using the prevalence-based approach, direct healtheare costs attributable to being obese have been shown to be relatively consistent from country to country contributing to between $2-7 \%$ of the total healthcare expenditures. ${ }^{56}$

There has been limited research conducted on the economic burden of obesity in Canada. ${ }^{46-47}$ One study however estimated the direct and indirect economic costs of obesity in Canada. The authors identified the relative risks of diseases associated with obesity from a meta-analysis of existing prospective studies using a prevalence-based approach. These risks were applied to the calculation of the PAF and then taken as a percentage of the healtheare costs of the associated diseases. Estimates were derived for both the direct healtheare expenditures and the indirect costs, which included the value of economic output lost due to illness, injury-related work disability, or premature death. The economic burden associated with obesity was estimated to be $\$ 4.3$ billion which included $\$ 1.6$ billion in direct costs and $\$ 2.7$ billion in indirect costs representing an average of $2.2 \%$ of the total healtheare costs in Canada. ${ }^{47}$ These calculations and estimates are based on various sources of information that are briefly described below and which have their own methodological limitations. A detailed discussion of these assumptions is outside the scope of the current thesis. 
The relative risk estimates used to conduct the PAF were based on a metaanalysis of original prospective studies that examined the relationship between exeess body weight and the risk of developing eertain diseases. The total healtheare costs associated with these specific diseases were obtained from the 1998 Economic Burden of Illness in Canada (Health Canada 2002) ${ }^{4}$ and the estimated prevalenee of overweight and obesity in Canada obtained from the Canadian Community Health Survey 2000/2001.

Another study used a similar approach and estimated the total 'direct' healtheare cost of obesity to be over $\$ 1.8$ billion annually, or approximately $2.4 \%$ of the total healtheare expenditures for all associated discases in Canada. The following obesityrelated medical conditions were included; type Il diabetes, hypertension, stroke, coronary artery disease, hyperlipidaemia, pulmonary embolism, gallbladder disease, postmenopausal breast eancer, colorectal caneer and endometrial cancer. ${ }^{\text {to }}$

The prevalence-based cost of obesity has been investigated internationally: in the USA, ${ }^{45,48-50,53}$ Australia, ${ }^{56}$ Finland,$^{54}$ France ${ }^{34}$ and the Netherlands. ${ }^{29}$ One of the first studies using a prevalence-based approach estimating the economic cost of obesity was conducted in the USA. This study focused on the following obesity-related medical conditions: type II diabetes, gallbladder disease, cardiovascular discase, hypertension and some cancers. The cost of treating each of these medical conditions was calculated by estimating that a proportion of eases occurred in obese individuals. The authors identified a certain proportion of these cases to be attributable to obesity. For example, $27 \%$ of CVD was diagnosed in obese individuals and 70\% of these CVD cases were attributable to obesity. Therefore, $19 \%(27 \%$ of $70 \%)$ of the estimated aggregate costs of treating 
CVD in the US could be attributed to obesity. The total costs attributable to obesity for these medical conditions were estimated at US\$39.9 billion representing $5.5 \%$ of the total cost-of-illness in 1986 . It was suggested that this may be an underestimate, as other obesity-related medical conditions not included in this estimate, such as musculoskeletal disorders could raise the estimate substantially. Indirect costs were estimated to be US\$23 billion. ${ }^{+8}$ It should be noted that the COI method for estimating the burden of illness is based on several assumptions and it is valid only as long as the information used to calculate the assumptions is correct.

The same author has conducted several other studies on the economic cost of obesity and associated medical conditions in the USA. A similar study was repeated in 1994, and included musculoskeletal disease as a medical condition associated with obesity. The earlier 1986 estimate of US $\$ 39.9$ billion increased to $\$ 45.8$ billion or $6.8 \%$ in 1994 of overall healtheare spending. ${ }^{49}$ A further study conducted in 1998 by the same authors, included several more obesity-associated medical conditions such as breast cancer, colon cancer and osteoarthritis. The estimate for direct costs increased to US\$51.6 billion in 1995 or to $5.7 \%$ of overall direct healtheare spending. ${ }^{50}$ In 1999 and using the revised BMI classification (BMI $>30)$, the total cost of obesity was estimated to be US $\$ 70$ billion or $7.0 \%$ of overall healthcare spending. ${ }^{51}$

A similar methodology was used in Australia. The comorbidities included were; type II diabetes, coronary heart disease, hypertension, gallbladder disease, breast cancer and colon cancer The authors estimated the healtheare cost of obesity to be AUS\$395 million for a period $1989-1990$ or $2.0 \%$ of overall healtheare spending. ${ }^{56}$ 
The same methodology was used in New Zealand to estimate healthcare costs associated with obesity. The obesity associated co morbidities included in the analysis were: type II diabetes, coronary heart disease, hypertension, gallbladder disease, breast cancer and colon cancer. Using a $\mathrm{BMI} \geq 30$, the authors estimated the cost to be NZ $\$ 135$ million in 1991 or $2.5 \%$ of overall healthcare spending. ${ }^{57}$

The total healthcare cost of obesity in France was estimated to be FF11.9 billion or $2.0 \%$ of overall healthcare spending for 1995 . This study included hypertension, gallbladder disease, breast cancer and colon cancer, osteoarthritis, genitourinary cancer, gout, myocardial infarction, dyslipidaemias as obesity-related medical conditions. The BMI $\geq 27$ was used in this study. ${ }^{39}$

There are differences in these countries in the way healthcare is funded and how it is delivered. The estimates of the total cost of obesity to the healthcare system, however are very similar, and may be due to the prevalence-based approach being used to estimate these costs.

The following limitations are inherent in the prevalence-based approach making comparisons across studies almost impossible: (i) the reliance on estimates of obesity prevalence and relative risks from published studies to calculate the population attributable risk fraction (ii) the basis for the choice of medical conditions known to be associated with excess body weight is not always clear and (iii) the use of different BMI cut-off points to classify overweight and obesity and (iv) the estimate of costs associated with diseases often provided by National governments of health agencies. In conclusion, the prevalence-based approach is a high level top-down approach relying on many 
estimates which may or may not be credible in order to determine the resources spent on a risk factor. It is not possible to determine what is happening at a grass roots level in terms of health services utilization and the burden placed on individual health professionals in the healthcare system.

\subsubsection{Individual-Level Approach}

As discussed earlier in the Introduction, few studies have used individual-level data to ascertain health services utilization and the direct cost of obesity. While population-based data are valuable, it is also important and useful to quantify the association of obesity and healthcare resource cost at the patient level. Linkage at the individual-level to healthcare utilization and subsequent costs allows for examination of the determinants or predictors of health services utilization while controlling for known

confounders such as patient health status, socioeconomic status, age and gender. ${ }^{56,80-85}$

A number of studies were conducted using healthcare records or other secondary sources to examine differences in overall healthcare costs by levels of BMI comparing overweight or obese persons to normal weight persons. ${ }^{40-42,45,56}$ These studies estimate healthcare cost across levels of BMI over a time period at the patient level averaging the cost per patient.

The influence of BMI on the utilization of healthcare services and associated average annual healthcare expenditures was assessed in a study on a population of 16,217 Americans that responded to the National Expenditure Survey. A strong positive relationship existed for both men and women between the level of BMI and healthcare utilization and expenditures. ${ }^{40}$ 
Studies using this approach have reported associations between self reported weight and height and the costs of outpatient, inpatient health services and annual healtheare costs. $^{+1-42}$ Of these two studies, one controlled for comorbid conditions, ${ }^{41}$ while the other did not adjust for any variables. The author of this latter study reported that the comorbid conditions were critical links in the pathway by which obesity leads to increased health serviees utilization, and therefore should not be included in the analysis. $^{+2}$

In the current thesis, an analysis of individual-level data allowing for the adjustment for confounders was conducted, rather than the more commonly used COI prevalence-based approach. The individual-level survey data were linked to physician and hospital databases. This linkage enabled the author to examine objective utilization and average healtheare costs over a five-year period and to identify whether obesity acts as an independent predictor of either health services use or direct healtheare costs. The ability to access health survey data representative of the provincial population and to link survey responses to objective health services utilization data allows for the control of potential eonfounders and may provide a better understanding and a more reliable estimate of the healtheare utilization and its associated cost by the obese population. 


\section{CHAPTER 3 Obesity Literature Review}

\subsection{Introduction}

The purpose of this chapter is to provide the context for a study on obesity and its impact on the healtheare system. It is well established that excess body fat is a risk factor for a number of chronic diseases ${ }^{17,21}$ but, until recently in historical terms excess body weight was not of concern either at a socictal or political level. Due to the increased availability of data on anthropometric measures, it is clear that there has been an increased prevalence of excess body weight in most populations. While it is clear that excess body weight has an associated impact on the health of the individual, it is not clear whether there is an associated impact on the healtheare system. Many rescarchers have reported that overweight and obese individuals are more likely to suffer from associated chronic discases thereby utilizing healtheare services more often than their lean counterparts. Future projections suggest that this population will place a large financial burden on the health system and many governments.

\subsubsection{Measures of Obesity}

The most common method for classifying excessive body weight at a population level is by measuring BMI endorsed by the World Health Organization (WHO). 17,21 Obesity is characterized by excessive body fat or adipose tissue $(\mathrm{BMI}>3())$ accumulated to such an extent that health may be adversely affected (see Table 3.1)." 
Table 3.I BMI and Associated Health Risks

Body mass index WHO classification

Risk of co-morbidities ${ }^{17.21}$

$<18.5$

Underweight

Increased

$18.5-24.9$

Normal

Average

$25-29.9$

Overweight

Increased

$30-34.9$

Class I obese

Moderate

$35-39.9$

Class II obeso

Severe

$\geq 40$

Class III obeso

Very severe 


\subsubsection{How is Body Fat Measured?}

Body fat can be estimated using direct and indirect measurements of body composition. Direct measurements such as magnetic resonance imaging (MRI), dual $\mathrm{x}-$ ray absorpitometry (DEXA) computed tomography (CT) or hydrodensitometry (underwater weighing) can accurately estimate body fat content. Indirect measurements of body fat or body composition obtained using non-invasive measures (c.g., waist circumference, waist-to-hip ratio, skin-fold thickness, bioimpedance, and body mass index) provide crude surrogate markers of body fatness.

\subsubsection{Direct Measurements of Body Fat}

Until recently the 'gold standard' for estimating body fat has been hydrodensitometry (underwater weighing). Dual-energy $x$-ray absorptiometry is now replacing hydrodensitometry as a gold standarl because of its high precision and its case of application for the subject. ${ }^{87}$ The DEXA body sean determines bone mineral eontent, body fat, and lean tissue mass by a precise computer controlled $x$-ray beam. The $x$-ray penetrates deep inside the body and is useful in determining visceral or intra-abdominal fat. In addition an MRI or a CT scan can provide high quality images of the inside of the body and an aceurate measure of visecral adiposity or intra-abclominal fat. ${ }^{8 x}$ These direce measures are used primarily in clinical settings and are not usually available for large population studies, due to their associated high operational costs and the practical difficulties involved in their application. However these direct methods play a very important role in rescarch. They are used to validate other indirect methods of estimating body fat such as waist circumference or BMI. ${ }^{89}$ 


\subsubsection{Indirect Measurements of Body Fat}

Anthropometric measures (e.g., waist circumference, waist-to-hip ratio, BMI) are the most common methods used to estimate individual body fat and body composition in the clinical setting and when conducting epidemiologic studies. The measurements of body circumference using waist circumference and waist-to-hip ratio have become more common over the last 20 years due to the interest in visceral fat (abdominal fat) as a potential independent risk factor for chronic disease. ${ }^{87}$ Waist circumference is a simple measurement unrelated to height that correlates closely with other indirect measurements of body fat such as BMI and waist-to-hip ratio, ${ }^{21}$ It is an estimate of visceral or intraabdominal fat mass, ${ }^{21,90,91}$ and upper body fat. ${ }^{92}$ Visceral fat is considered to be metabolically different from subcutaneous fat (i.c., all over fat) in its responsiveness to dietary changes and its metabolic and hormonal output. Excessive visceral fat has been associated with the metabolic syndrome. This syndrome includes a collection of metabolic disturbanees such as hypertension, dyslipidaemia, impaired glucose tolerance and insulin resistance which can lead to the development of cardiovascular discase."?

Researchers report that waist eircumference is a more accurate indicator of increased health risk when compared to BMI and should be included in assessments of

obesity. ${ }^{4-4)}$ In addition; changes in waist circumference reflect changes in risk factors for cardiovascular discase, $" 4$ and other forms of chronic discase. Health canada in 2003 adopted specific cut-off points for abdominal girth measurement in both men and women. For men, a waist girth greater than $102 \mathrm{~cm}$ or 40 inches and for women a waist girth greater than $88 \mathrm{~cm}$ or 35 inches, is associated with an increased risk of type Il diabetes, 
coronary heart disease and hypertension. ${ }^{97,98}$ There is difficulty in drawing international comparisons using these measurements. Canada accepts these recommended thresholds for waist girth, however other countries do not. Although the accumulation of abdominal fat is associated with a number of comorbidities, cross-sectional population studies conducted in Africa, the Caribbean, the United States and Canada report that the relationship between waist circumference and chronic disease varies by region. ${ }^{(9)-102}$ Consequently, international comparisons are limited due to the cut-off points being population specific and dependent on the regional prevalence of obesity and cardiovascular risk factors. An additional challenge for researchers is the proposal of different cut-off points for waist girth based on a person's age and sex. ${ }^{21}$ When defining the problem of overweight and obesity, the WHO in 1995, recommended that waist circumference should be measured concurrently with BMI. ${ }^{103}$

It has been suggested that combining the waist-to-hip ratio with the measurement of waist circumference will more accurately reflect health risk, especially in women. The waist-to-hip ratio is often equated with the apple and pear body shape. In general men tend to be apple shaped and women pear shaped. Typically men distribute fat around their abdomen and women deposit high amounts of fat around their hips, buttocks and thighs. The additional measurement of the waist-to-hip ratio indicates the degree of fat accumulation around the hips. Extra fat around the hips may be protective of adverse health consequences for the individual. ${ }^{10+4}$

The waist-to-hip ratio is not often used as a measure of obesity. However, a recent case control study published in the Lancet in 2005 and conducted by the Interheart Study 
Group suggested that the waist-to-hip ratio may be more accurate when determining the risk of myocardial infarction. The authors compared the waist-to-hip ratio with BMI and the odds of an individual having experienced a myocardial infarction, for 27098 participants across 52 countries. Body mass index showed a modest and graded association with myocardial infarction. When comparing the top quintile to the bottom quintile before adjustment, the resulting odds ratio (OR) was 1.44 [95\% CI (1.32-1.57)]. Adjusting for the measure of the waist-to-hip ratio substantially reduced the OR to 1.12 [95\% CI (1.03-1.22)]. Having adjusted for other risk factors, the relationship between $\mathrm{BMI}$ and myocardial infarction was not significant; OR 0.98 [95\%CI $(0.88-1.09)]$. For waist-to-hip ratio, the odds ratios for every successive quintile were significantly greater than that of the previous one, adjusting for age, sex, region, and smoking (2nd quintile: OR 1.15 [95\% CI (1.05-1.26)]; 3rd quintile: OR 1.39 [95\% CI(1.28-1.52)]; 4th quintile: OR $1.90[95 \%$ CI (1.74-2.07)]; and the 5th quintile: OR 2.52 [95\% CI (2.31-2.74)]. The waist-to-hip ratio showed a graded and highly significant association with myocardial infarction risk worldwide suggesting obesity based on waist-to-hip ratio as a replacement for BMI increased the accuracy of estimating the likelihood of myocardial infarction. ${ }^{105}$ One major limitation of this study is its retrospective case control study design, which is weak in its ability to suggest a causal relationship. In addition, this method of estimating body fat is associated with measurement error, due to the unreliable measurement of both the hips and the abdomen.

Using skin-fold measurements, the percentage of subcutaneous body fat can be measured by body-fat calipers. This instrument measures body fat in specific sites such as 
the triceps, the shoulder blade and waist. The measurement error associated with this technique limits its use in research. As well, these measurements do not provide information about the existence of abdominal or intramuscular fat. ${ }^{106}$

\subsubsection{Body Mass Index and Associated Health Risk}

The most common non-invasive method for indirectly estimating body fat and its associated health risks is through the calculation of the Quetelet's or BMI ${ }^{21}$, defined as weight in kilograms divided by height in metres squared:

\section{BMI = weight $(\mathrm{kgs}) /$ height $\left(\mathrm{m}^{2}\right)$}

According to the WHO, BMI is the most appropriate measure by which weight adjusted for height can be related to health outcome. The BMI index is easy to calculate and correlates strongly with direct measures of fatness or adiposity $(r=0.82-0.91)$ in adults. ${ }^{20}$ BMI has greater reproducibility than other measures of adiposity such as skin fold thickness, waist circumference and waist-to-hip ratio. ${ }^{21}$

Body mass index is simple to calculate and is a useful measure for indirectly estimating body fat. The graded BMI classification system of underweight, normal, overweight and obese permits (a) comparisons of weight status within and between populations (b) identification of individuals and groups at increased risk of morbidity and mortality (c) priorities to be identified for intervention at individual and community levels and (d) a basis for the evaluation of interventions. 
Body mass index provides a useful crude population-level measure of obesity. This measure can be used to estimate prevalence of obesity within a population and the associated health risks. Body mass index provides a reasonably reliable screening tool that is easy and inexpensive to use on large populations. ${ }^{21}$

\subsubsection{Body Mass Index as a Measure of Body Fat}

Although BMI is the most commonly used method to classify weight status and health risk, there are many disadvantages inherent in its calculation. Different ethnic groups have substantially different proportions of body fat at the same value of BMI. ${ }^{21}$ For example, the ratio of fat to lean tissue is highest in Indian people, intermediate for Chinese people and low for Caucasians. ${ }^{107}$ In addition Polynesians have a relatively high proportion of lean tissue. ${ }^{108-109}$ It is also documented that women have substantially more fat tissue than men, ${ }^{110}$ and that both men and women lose lean tissue at different rates throughout the life cycle. ${ }^{111}$ As well, the BMI values associated with increased health risk vary according to ethnicity. ${ }^{112-113}$

Body Mass Index does not distinguish between increased body weight due to adiposity or fluid retention. As an index, BMI fails to distinguish between muscle, body fat, or bone. Men and women with the same BMI may have a different percentage of body fat or fat mass compared to lean mass. As well, BMI does not indicate the location or the distribution of excess fat. BMI does not account for the wide variation in body fat distribution and may not correspond to the same degree of fatness or associated health risks in different individuals or populations. ${ }^{103,114}$ Obese individuals with excess fat in the intra-abdominal area are at risk for the development of adverse health consequences. ${ }^{115}$ 
The calculation of BMI measures overall fat, but will not provide information on the location of the fat. Also, when measuring individuals who demonstrate extreme height or muscle mass, the accuracy of the BMI value may vary. A health professional using BMI as an indicator to measure body fat, may conclude incorrectly that an individual is obese. Although, a high BMI may suggest an increased risk of an associated discase, this measure may also indicate a very muscular individual. An individual with a high proportion of muscle relative to height may have a high BMI value, yet not be obese. ${ }^{16}$ As well BMI is less accurate for estimating body fat in very short individuals. 117

\subsubsection{Health Canada and Changing BMI Guidelines}

In 2003, Health Canada updated its body weight classification system based on research conducted by the WHO. This classification has been widely adapted intemationally. ${ }^{118}$ There were some similarities between the old and new classitication system, however, the differences in the updated version are significant (Table 3.2). The updated classification system included changes to certain cut-off points in the BMI categories. The introduction of a waist circumference as a measure of body fat and associated health risk was also introduced. The range for normal weight has increased to include a BMI in the range of 18.5-24.9 whereas in the old system normal weight included BMI's in the range of $20-25$. The relationship between BMI and mortality was examined in a representative sample of the American population. ${ }^{22}$ Researchers suggested that a j-shaped relationship existed between BMI and mortality (having adjusted for underlying illness) with increased risk of mortality at a BMI below 18.5 relative to a BMI in the normal range of 18.5 - 24.9. In 1988, researehers suggested that 
a BMI between 25 and 27 led to an increased risk of health problems in some individuals, while a BMI $>27$ was associated with increasing risk of developing health problems in many individuals. Since then, more research has been conducted on the relationship between BMI and mortality and morbidity risk. ${ }^{17,22,86}$ The mortality curves generated from these studies illustrate the relative risk of mortality begins to inerease at a BMI of 25 , and there is a marked increase of mortality risk at a BMI $>30$ and a $\mathrm{BMI}<18.5$. Based on these findings, the WHO deseribes the BMI range of 25 to 29.9 as preobese. In Canada, this BMI range is described as overweight. Both the WHO and Health Canada use a $\mathrm{BMI}>30$ to classify individuals as obese. The obese elassification is further broken down into three groups (Table 3.2). 
Table 3.2 Comparison of BMI Classification Systems 1998-2003

\begin{tabular}{|c|c|c|}
\hline Canada $1988^{118}$ & WHO $2000^{21}$ & Canada $2003^{118}$ \\
\hline Zone A BMI $<20$ & Underweight & Underweight \\
\hline $\begin{array}{l}\text { May be associated with } \\
\text { health problems in some } \\
\text { people }\end{array}$ & $\mathrm{BMI}<18.5$ & $\mathrm{BMl}<18.5$ \\
\hline Zone B: BMI 20-25 & Normal range & Normal range \\
\hline $\begin{array}{l}\text { Good weight for most } \\
\text { people }\end{array}$ & $18.5-24.99$ & $18.5-24.99$ \\
\hline Zone C: Between 25 and 27 & Preobese & Overweight \\
\hline $\begin{array}{l}\text { May lead to health } \\
\text { problems in some people }\end{array}$ & $25.0-29.99$ & $25.0-29.99$ \\
\hline Zone $\mathrm{D}: \mathrm{BMI}>27$ & Obese Class I & Obese Class I \\
\hline \multirow{5}{*}{$\begin{array}{l}\text { Increasing risk of } \\
\text { developing health problems }\end{array}$} & $30.0-34.99$ & $30.0-34.90$ \\
\hline & Obese Class 11 & Obese Class II \\
\hline & $35.0-39.99$ & $35.0-39.99$ \\
\hline & Obese Class III & Obese Class III \\
\hline & $\geq 40.0$ & $\geq 40.0$ \\
\hline
\end{tabular}


Nevertheless, international convention, represented by a major WHO Technical Consultation endorsed the use of a common BMI scheme for adults irrespective of sex and age. ${ }^{21}$ As a result, in 1995 the WHO, in collaboration with the International Obesity Task Force designed a global BMI classification system for the identification of underweight, normal weight, overweight and obese individuals. This new classification system allows for meaningful comparisons within and between populations.

\subsection{Obesity and its Burden of Illness}

Obesity is often referred to as a chronic disease, that is purported to be related causally to serious medical illnesses. ${ }^{119}$ These chronic illnesses may not be fatal but may be debilitating in nature and have an adverse effect on an individual's quality of life. As well, these obesity related illnesses may increase the risk of mortality. Obesity increases the risk of the development of several chronic conditions such as cardiovascular diseases, hypertension, coronary heart disease (CHD), cerebrovascular disease, cancer and type II diabetes. As well, obesity is associated with metabolic syndrome and endocrine disturbances and other debilitating conditions. In industrialized countries, it has been reported that obesity has a psychosocial impact on individuals through prejudice, bias and social stigma. ${ }^{21}$

\subsubsection{Hypertension}

Many studies have reported a positive association between BMI and hypertension.

As BMI increases, the risk of developing hypertension increases. ${ }^{120-123}$ Based on the Framingham study, a large longitudinal cohort study in the USA started in the 1950's in the USA, the relationship between BMI and blood pressure has been examined over time. 
Authors have reported an increase of $6.5 \mathrm{mmHg}$ in blood pressure with every $10 \%$ increase in body weigh for this population ${ }^{120}$ and other longitudinal studies have demonstrated similar results. ${ }^{121-123}$

In addition cross-sectional studies have demonstrated that the prevalence of hypertension is increased in overweight and/or obese individuals. Using cross-sectional data from the Third National Health and Nutrition Examination Survey (NHANES III), the age-adjusted prevalence of hypertension was $42 \%$ and $38 \%$ in obese men and women respectively. This was twice as high as the reported prevalenee of hypertension for men and women classified as normal (BMI $<25)$ which was approximately $15 \%$ in both men and women. In this study, hypertension was defined as systolic blood pressure $>140 \mathrm{~mm}$ $\mathrm{Hg}$, diastolic blood pressure $>90 \mathrm{~mm} \mathrm{Hg}$, or the need for hypertensive medication. ${ }^{24}$

\subsubsection{Coronary Heart Disease}

The authors of a meta-analysis, including 21 cohort studies reported on at combined sample size of 300,000 individuals and their related 18,000 CHD events. The adverse effects of high blood pressure and cholesterol levels associated with overweight and obesity accounted for $45 \%$ of the increased CHD risk. Adjusting for age, physical activity and smoking, the relative risks and $95 \%$ confidence intervals for moderate overweight and obesity compared with normal weight were 1.32 [95\% CI (1.24-1.40)] and $1.81[95 \% \mathrm{CI}(1.56-2.21)]$ respectively. The relative risk associated with a 5 -unit BMI increment was 1.29 [95\% CI (1.22-1.35)]. Gender was not examined separately in this review. ${ }^{124}$ 
Longitudinal studies report an increasing relative risk of developing CHD with increasing levels of BMI. ${ }^{125,126}$ Findings from the Nurses Health Study, a large scalc prospective study of women $(\mathrm{n}=115,886)$, suggest that the relative risk of non-fatal myocardial infarction, increased as BMl increased. Women in the heaviest weight category (BMI $>29)$ were approximately 3.5 times more likely to develop CHD as compared to women in the lightest weight category (BMI of $<21$ ). ${ }^{126}$ Another study examined the relationship between BMI and CHD risk factors in 314 non diabetic, normotensive, healthy volunteers, and reported obesity to be a powerful predictor of CHD risk. ${ }^{125}$

\subsubsection{Cerebrovascular Disease}

The authors of a prospective cohort study using the Nurses Health Survey examined the associations between BMI and weight changes with the risk of stroke in women. The study population $(\mathrm{n}=116,759)$, included women 30 to 55 years of age in 1976, frec from diagnosed CHD, stroke, and cancer. During I 6 ycars of follow-up, it was reported, 866 total strokes, including 403 ischemic strokes and 269 hemorrhagic strokes occurred. Using multivariate analysis, adjusting for age, smoking, postmenopausal hormone use, and menopausal status, women with increasing (BMI $\geq 27$ ) had significantly increased risk of ischemic stroke. The authors reported increasing relative risks of $1.75[95 \% \mathrm{CI}(1.17-2.59)]$ for individuals with a BMI between 27 to $28.9 \mathrm{~kg} / \mathrm{m}^{2}$; 1.90) $[95 \% \mathrm{Cl}(1.28-2.82)]$ for individuals with a BMI between 29 to $31.9 \mathrm{~kg} / \mathrm{ml}^{2}$, and 2.37 $[95 \% \mathrm{Cl}(1.60-3.50)]$ for individuals with a BMI $>32 \mathrm{~kg} / \mathrm{m}^{2}$ (P for trend $\left.<.(0) 1\right)$. These groups were compared to individuals with a $\mathrm{BMl}<21 \mathrm{~kg} / \mathrm{m}^{2}$. The risk of fatal and non- 
fatal ischemic stroke was more than twice as high in obese persons than in lean persons and the risk of stroke appeared to increase with increasing BMI. ${ }^{127}$

The association between excess weight and increased risk of stroke in men does not appear to be as strong as in women. In one study, the association of BMI with stroke incidenee was examined. As well, the association between abdominal obesity and stroke incidence was examined using the waist-to-hip ratio. This study examined 28,643 US male health professionals, aged 40-75 years in 1986. This group did not have a history of cardiovascular disease or stroke. In a 5-year follow-up, there were 118 cases of stroke, of which 80 were ischemic. Comparing men in the lowest and highest quintile of BMI, the results were not significant. In contrast, the age-adjusted relative risk for extreme quintiles of waist-to-hip ratio was 2.33 [95\% CI (1.25-4.37)] was found to be significant. This relative risk was not substantially altered in a multivariate model including BMI, height, and other potential risk factors. When waist circumference alone was used as a measurement of excess body fat, there was no significant relationship with men in the highest quintile $(>102 \mathrm{~cm})$ and the incidence of stroke compared with men in the lowest quintile $(\leq 88 \mathrm{~cm})$. These results suggest that abdominal obesity measured by waist-to-hip ratio ratio, but not BMI or waist circumference, predict the risk of stroke in men. In addition for men, the risk of developing venous stasis, deep vein thrombosis and pulmonary embolism also increased with abdominal obesity. ${ }^{128}$

\subsubsection{Cancer}

There is evidence to suggest overweight and obesity are associated with an increased risk of various types of cancer such as esophagus, gallbladder, colon, breast, 
endometrial, uterus, cervix and prostate. Epidemiologic studies have found a direct relationship between BMI and colon cancer in both men and women. In addition the risks of mortality from endometrial cancer increases with obesity and weight gain after 18 years of age. ${ }^{129-132}$

Studies have shown a complex relationship existing between obesity and the risk of breast cancer and its prognosis. A review on obesity and the prognosis of breast cancer in 2006, examined the findings from a number of systematic reviews, meta-analyses and individual cohort studies. The authors reported a consistent, independent and positive association between obesity and the development of breast cancer risk in postmenopausal women. Literature supports the association between obesity and the poor prognosis of breast cancer in both pre and post menopausal women. ${ }^{132}$

\subsubsection{Diabetes}

A strong association between obesity and type II diabetes has been observed in a number of studies. ${ }^{133-136}$ Researchers suggest the increasing prevalence of obesity is related to the increasing incidence of type $\mathrm{Il}$ diabetes that has oceurred in both Europe. the USA and Canada. ${ }^{133}$ Data from a cohort study of 51.529 U.S male health professionals 40 to 75 years of age were analyzed. These male health professionals had completed biennial questionnaires sent out between 1986 and 1992. The relative risks of developing type Il diabetes were analyzed. Compared to men with a BMI $\cdot 23$, men with a $\mathrm{BMI}>30$ had a relative risk of 11.0 , which increased to a relative risk of 42.1 with a $\mathrm{BMI}>35^{1.34}$ 
Authors of the longitudinal Nurses Health Study, reported that the risk for women developing type 11 diabetes started to increase when BMI values exceeded a value of $22.1^{135}$ A study using nationally representative cross-sectional survey data from NHANES III, reported on the prevalence of type II diabetes. Comparing individuals with a BMI $(18.5<\mathrm{BMI}<25)$, to those with a BMI value $\geq 40$, the prevalence ratio for type $1 \mathrm{I}$ diabetes for men was 18.1 [95\% Cl (6.7- 46.8)] and for women 12.9 [95\% (Cl 5.728.1 1)]. ${ }^{136}$ The evidence presented shows a strong association between obesity as a risk factor the development of type II diabetes, in both men and women.

\subsubsection{Endocrine and Metabolic Disturbances}

Obesity is alssociated with endoerine disturbances such as elevated cholesterol levels and metabolic disturbances such as the metabolic syndrome. Overweight and obesity contribute to elevated cholesterol levels and or abnormal lipid profiles (e.g., high levels of LDL cholesterol, triglycerides and low levels of HDL cholesterol). Studies report higher levels of circulating cholesterol in subjects with higher BMI's or in those classified as obese compared with individuals with lower BMl values (i.e., BMl $<$ 25). ${ }^{125.136}$ Mean values for total cholesterol, triglycerides and LDL cholesterol increased with each increasing BMI category from normal, overweight to obese. ${ }^{137}$ Using NHANES III (1988-1994) data, the authors reported there was an increase in the prevalence of hypercholesterolemia (total blood cholesterol $>240 \mathrm{mg} / \mathrm{dl}$ or $6.21 \mathrm{mmol} / \mathrm{l}$ ) in men with increasing BMI. ${ }^{138}$

Obesity is associated with the existence of a condition described as "the metabolic syndrome" or "syndrome $X^{-129}$ characterized by a cluster of metabolic risk factors 
identified in the development of CHD. In the USA, the Adult Treatment Panel (ATP III) on detection, evaluation and treatment of high blood cholesterol, issued guidelines for the clinical identification of the metabolic syndrome. ${ }^{138}$ An individual may be diagnosed as having the Metabolic Syndrome if three or more of the following conditions are present: abdominal obesity, hypertriglyceridemia. low HDL cholesterol, hypertension and high fasting glueose. Since this classification has been published, a number of studies have been conducted to estimate the prevalence of the metabolic syndrome in specific populations.

In the USA, using data from the NHANES III and based on the above ATPIII criteria, the prevalence of the age-adjusted Metabolic Syndrome was estimated to be

approximately $24 \%{ }^{134}$ A study published on 6406 men and 6475 women in Canadal using the same guidelines reported the prevalence of the metabolic syndrome to be $14.4 \%$ in adults 18 to 64 years of age. In this study, the prevalence of the metabolic syndrome increased from $6.7 \%$ for individuals 20 to 30 years of age to $43 \%$ for individuals 60 to 70 years of age although there was little difference in the prevalence between men and women. ${ }^{140}$

\subsubsection{Obesity and Debilitating Conditions}

Obesity increases the risk of developing or aggravating debilitating conditions such as osteoarthritis due to the excessive weight placed upon joints. A number of studies have reported evidence to link obesity with a variety of musculoskeletal disorders ranging from osteoarthritis to joint pain. The risk of developing osteoarthritis in weight-bearing joints is increased in overweight and obese persons. The knees are often involved because 
more body weight is exerted across the knees during weight bearing activity than across other joints such as the hips. ${ }^{141}$ There is a stronger relationship between body size and osteoarthritis in women as compared with men. Small increases in body weight in women can promote osteoarthritis. In a study of twins, symptomatic or asymptomatic lower extremity osteoarthritis was found in individuals who were only 3 to 5 kilograms heavier than their twin sibling. For each kilogram increase in body weight, the risk of developing osteoarthritis increased by 9 to $13 \%$. For each $\mathrm{kg}$ increase in weight the increased likelihood of developing different osteoarthritis traits was: tibiofemoral osteophytes OR 1.14 [95\% CI (1.01-1.28)], patellofemoral osteophytes OR 1.32 [95\% CI (1.09-1.59)] and patellofemoral narrowing OR $1.15[9.5 \% \mathrm{CI}(1.01-1.30)]^{142}$

\subsection{Obesity and Psychological Health}

Obesity experienced in childhood and during adoleseence has an adverse effect on long-term social and economic outeomes in adulthood. These factors include the level of income and educational attainment. ${ }^{143}$ One study followed 370 overweight subjects for seven years. Women who had been overweight at the beginning of the study, had completed 0.3 years less of schooling; [ $95 \%$ CI ( 0.1 to 0.60$)]$, were $20 \%$ less likely to be married [95\% CI(13-27\%)], had \$ 6,710 houschold income less per year [95\% Cl $(\$ 3,942-9,478)]$ and had $10 \%$ higher rates of houschold poverty [95\% CI (4- $16 \%)]$ compared to women who had not been overweight. These results were independent of their base-line socioeconomic status and aptitude - test scores.

Another study found men who had been overweight as adoleseents were $11 \%$ less likely to be married later in life [ $95 \% \mathrm{Cl}(3-18 \%)]$. The authors concluded that being 
overweight during adolescence had important social and economic consequences. The authors suggested that discrimination against overweight persons might account for this finding. ${ }^{14+}$

\subsection{Obesity and Mortality}

Controversy exists over the relationship between obesity and premature mortality. A number of studies have found a " $u$ ' or ' $j$ ' shaped association between BMI and mortality, showing a higher mortality rate at the upper and lower BMI ranges. 22-23.145-146 A gradual increase in mortality with increasing weight has also been demonstrated in some studies, ${ }^{147-1+8}$ while other research does not demonstrate any association. ${ }^{14}$

The relationship between BMI and mortality data was examined in a large prospective study of more than one million adults in the USA. Over a period of 14 years 457,785 men and 588,369 women were followed. A j-shaped relationship between BMI and mortality was observed. In healthy non-smokers, the lowest risk of mortality was seen in males with a BMI in the range of $23.5-24.9 \mathrm{~kg} / \mathrm{m}^{2}$ and in females with a $\mathrm{BMI}$ in the range of $22.0-23.4 \mathrm{~kg} / \mathrm{m}^{2}$. A high BMI was predictive of mortality from cardiovascular disease in men RR 2.90 [95\% CI (2.37-3.56)]. Heavier men and women in all age groups had an increased mortality. ${ }^{22}$

The expected number of years of life lost (YLL) between normal, overweight and obese groups in Caucasian and black Americans was compared. Years of life lost being the difference in life expectancy between normal, overweight and obese groups. When several large USA - based population surveys were analyzed, it was found that race and sex differences were observed in YLL. Among the white population, a j-or u-shaped 
association was found between overweight or obesity and YLL. The optimal BMI range was between 23 and 25 for the white population and between 23 and 30 for the black population. These BMI ranges were associated with the least YLL or greatest longevity for both groups. For any given degree of overweight, younger adults had greater YLL than did older adults. The maximum YLL for individuals between 20 and 30 years of age classified with severe obesity (BMI $>45)$ was 13 years for white men and eight years for white women. For men, this represented a $22 \%$ reduction in the life span. Among black men and women older than 60 years of age, overweight and moderate obesity were generally not associated with increased YLL and only severe obesity resulted in YLL..3

A more linear relationship has been demonstrated between BMI and mortality and is supported by authors who have used prospective longitudinal studies. The Nurses Health Study for example followed 116,000 women in the USA over a 17 -year period. When biases were controlled for, a continuous positive association existed between BMI and mortality. This finding was supported by the largely positive relationship found between increasing body weight and conditions such as CHD, hypertension and type II diabetes. ${ }^{147}$ It is proposed that higher mortality rates at the underweight of the BMI scale or at a BMI $<18.5$ are artificial. The increased mortality at this end of the BMI range, may result firom the lack of adjustment of other factors known to increase the risk of mortality such as eigarette smoking or underlying comorbidities. ${ }^{\text {th }}$ Severe obesity or morbid obesity is defined as a $\mathrm{BMI} \geq 35$ or $\geq 40 \mathrm{~kg} / \mathrm{m}^{2}$ respectively. A systematic review published recently examined the relationship between severe obesity and mortality. Patients with severe obesity (BMI $\geq 35)$ did not have an increased risk of total all-cause 
mortality RR 1.10 [95\% CI (.87-I.41)]), but had the highest increased risk for cardiovascular mortality $\mathrm{RR} 1.88[95 \% \mathrm{CI}(1.05-3.34)] .{ }^{150}$ This may be due to the specific chronic conditions associated with excess weight and with an increased workload of the heart. Another explanation for these findings is reverse causality. That is an individual suffering from heart disease may be more likely to lead a sedentary life thus leading to increased risk of mortality. Severe obesity has also been associated with sudden cardiovascular death due to its association with obstructive sleep apnea syndrome. Sleep apnca is a breathing disorder characterized by interruptions of breathing during slecp. There are two types of sleep apnea central and obstructive. Central sleep apnea oceurs when the brain fails to send the right signals to the breathing museles to continue breathing and obstructive sleep apnea occurs when air cannot flow in or out of an individual's nose and mouth. Many episodes of obstructive sleep apnea oceur every night and, it is thought, increase the risk of hypertension, heart problems and death. ${ }^{151}$

\subsection{Obesity and Disease - a Causal Relationship?}

The preceding review outlines some of the adverse health risks associated with overweight and obesity suggesting a causal relationship. Evidence suggests that with moderate weight loss, the ill-effects of excess weight can be reversed. A cochrane systematic review was conducted to determine the effects of weight loss on chronic diseases. ${ }^{152}$ The Cochrane Collaboration is an international not-for-profit, independent organization producing information about the effects of healtheare interventions. This organization disseminates systematic reviews of healtheare interventions and promotes the search for evidenee in the form of elinical trials and other studies of interventions. 
This review identified 18 randomized controlled trials including 2611 hypertensive participants with an average body weight of $84 \mathrm{~kg}$. This data suggested weight loss in the range of $4 \%$ to $8 \%$ of total body weight through weight-reduction diets, produced an average reduction in systolic blood pressure of $3.0 \mathrm{mmHg}$ and these findings are consistent with earlicr reviews. ${ }^{153}$

Another study reported weight loss resulted in a significant lowering of both resting and exercise blood pressure in morbidly obese subjects. ${ }^{154}$ In addition a meta analysis of intervention trials reported losing excess weight improved blood lipid profiles with a fall in total serum cholesterol and triglyecride levels and an increase in high density lipoprotein (HDL) concentrations. ${ }^{155}$

Experimental evidence is emerging demonstrating a reduction in a small proportion of body weight may by effective in reducing adverse disease outcomes. These experimental studies provide the strongest evidence of a causal relationship between level of BMI and the development of discase. ${ }^{156}$

\section{6 Can Overweight or Obese Individuals be Healthy?}

Although much of the published literature focuses on the association between obesity as a risk factor for many adverse health conditions, several studies have been published recently demonstrating that lower rates of cardiovascular mortality are seen in older age groups who are overweight or obese but considered to be physically active when compared to inactive normal weight individuals. ${ }^{157-159}$ 


\subsection{Epidemiology of Obesity}

The prevalence of obesity has increased in many countries over the past 25 years. As well the average BMI values and the prevalence of obesity vary with age, sex, race, geography and socioeconomic status in these countries. Figure 3.1 illustrates the current prevalence of obesity in the Organization of Economic Development (OECD) member countries.

\subsubsection{Global Trends}

Of the OECD member countries, the United States has the highest prevalence of adult obesity (30.6\%) followed by Mexico (24.2\%) and the United Kingdom (22.4\%). Canada is ninth on the list with a prevalence of obesity of $14.9 \%$. Korea and Japan report the lowest prevalence of obesity at $3.2 \%$ and $3.6 \%$, respectively. 


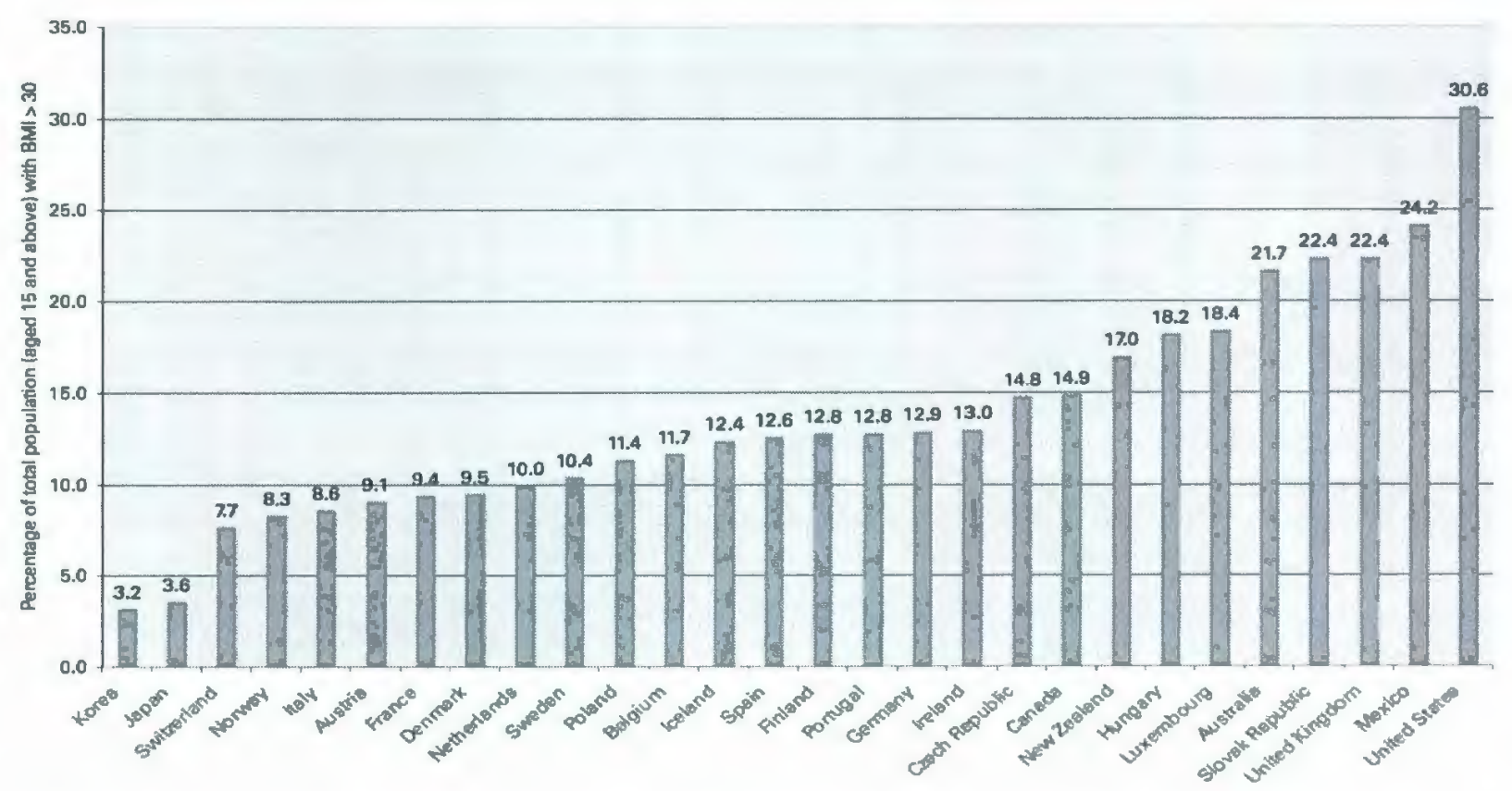

Figure 3.1 Global Prevalence of Obesity in Adult Populations $\left(\mathrm{BMI}>30 \mathrm{~kg} / \mathrm{m}^{2}\right)$ in $\mathrm{OECD}$ Member Countries

Copyright OECD Health Data 2006 (permission not required to use this graph) 
Global studies, Multinational Monitoring of Trends and Determinants in Cardiovascular Discase (MONICA) conducted by the WHO reported that on average a greater proportion of women are obese compared to men, while a greater proportion of men are overweight compared to women. Some longitudinal data is available from national surveys on the prevalence and the increasing rate of obesity. The prevalence of obesity in the USA doubled between 1980 and 2002 and is continuing to rise. ${ }^{1(0)-1(0)}$ During a similar time period, Canada experienced approximately a three-fold increase in the prevalence of obesity. ${ }^{162}$

\subsubsection{National Trends}

Published data on the prevalence of obesity in Canada from York University reported dramatic increases in the prevalence of obesity oceurring at both the national and provincial levels. ${ }^{25}$ The data for this report was obtained from five population-level surveys collecting self-reported heights and weights in Canada. Obesity was defined as $\mathrm{BMl} \geq 30 \mathrm{~kg} / \mathrm{m}^{2}$ and the sample was representative of the Canadian population for each year the survey was eonducted. The results from the Canadian surveys showed an overall increase in the prevalence of obesity. The national prevalence of obesity more than doubled over the 14-year period from $5.6 \%$ in 1985 to $14.8 \%$ in 1998 . Over the 14 -year span considered, all provinces experienced increases in the prevalence of obesity. By 1998, only Quebec and British Columbia demonstrated obesity prevalence below 15\%. Data for the Northwest Territories, the Yukon and Nunavut was not available.

The prevalence of adult obesity in Canada was $16.3 \%$, based on self reported heights and weights. These findings were reported from data published using the 2005 
Canadian Community Health Survey. ${ }^{25.27}$ The comparative prevalence of adult obesity in NL, using this survey was approximately $27 \%{ }^{27}$ (Table 3.3). The 2004 Canadian Community Health Survey: Nutrition, a survey conducted in between regular survey years collected data on direct measures of heights and weights. According to this survey the National prevalence of adult obesity was $24 \%$ compared to provincial prevalence of $34 \%$ in NL. 
Table 3.3 The Prevalence of Adult Obesity in Canada based on self reported heights and weights 1985 to 2005

\begin{tabular}{|c|c|c|c|c|}
\hline \multirow[b]{2}{*}{ Year of survey } & \multicolumn{2}{|c|}{ Canada } & \multicolumn{2}{|c|}{ NL } \\
\hline & Preva & $\%(95 \% \mathrm{CI})$ & Prevalence\% & $(95 \% \mathrm{CI})$ \\
\hline 1985 & $5.6^{*}$ & & \multicolumn{2}{|c|}{ No data available } \\
\hline 1990 & $9.2 *$ & & \multicolumn{2}{|c|}{ No data available } \\
\hline 1994 & 12.7 & $(12.0,13.3)$ & 16.4 & $5,19.4)$ \\
\hline 1996 & 11.9 & $(11.5,12.4)$ & 16.2 & $(6,18.9)$ \\
\hline 1998 & 14.1 & $(13.4,14.9)$ & 18.7 & $1.21 .2)$ \\
\hline 2001 & 15.3 & $(14.9,15.6)$ & 21.7 & $8-23.6)$ \\
\hline 2003 & 15.8 & $(15.5,16.2)$ & 21.4 & $4-23.5)$ \\
\hline 2005 & 16.3 & $(16.0,16.7)$ & 26.7 & $3-29.0)$ \\
\hline
\end{tabular}

1985, 1990 Health Promotion Survey, 1994-1998 National Population Health Survey, 2001-2005 Canadian Community Health Survey, * Confidenee Intervals were not available for these data sets 
Although the current study did not include children, there is an emerging childhood obesity epidemic being reported. ${ }^{163-164}$ Over a 15-year time period, there has been a significant increase in the prevalence of overweight and obesity in Canadian children 7 to 13 years of age. Three national surveys were conducted between 1981 and 1996 reporting the pereentage of overweight boys increased from $15 \%$ to $29 \%$ and the pereentage of overweight girls increased from $15 \%$ to $24 \%$. These surveys also reported the prevalence of childhood obesity increased from $5 \%$ to $13 \%$ for boys and from $5 \%$ to $11.8 \%$ for girls. $^{163}$ Another study in Canada, reported $33 \%$ of boys and $26 \%$ of girls were considered to be overweight, and $10 \%$ of boys and $9 \%$ of girls were considered to be obese. These findings suggest that over this time period, the prevalence of childhood overweight and obesity in boys had doubled and is increasing in girls. ${ }^{164}$

There is limited data on the prevalenec of overweight and obesity in younger preschool children in Canada, however provincial data suggests there may be an emerging ehildhood obesity epidemic oceurring. A study published in the CMAJ reported $25 \%$ of NL children between the ages 3.5 and 5 participating in their Preschool Health Check were either overweight or obese. These results were based on direct measurements. ${ }^{165}$ Another study on pre-school children in NL found similar results. ${ }^{\text {Ito }}$ These findings may have implications for the healtheare system due to the increasing prevalenee of higher weights seen at an early age in ehildren and the potential association for adverse health consequences. 


\subsubsection{Provincial Trends}

Canada has experienced an increase in the prevalence of obesity, however the increase is not uniform from province to provinee. 25 The prevalence of obesity tends to decrease as one moves from east to west in Canada. The east coast of Canada, in particular the Atlantic Provinees, report a higher prevalenee of obesity, whereas the lowest prevalence is reported in western Canadia. The Atlantic Provinees demonstrate a higher than average prevalence of obesity when eompared to the other provinees.

The provincial information on the prevalence of adult obesity was obtained from the National Population Health Surveys and the more recent Canadian Community Health Surveys conducted by Statistics Canada. According to the 1995/1996 National Population Health Survey and the 2004/2005 Canadian Community Health Survey, the overall prevalence of adult obesity in NL increased from $17 \%$ to $24 \%$ of the total population compared to the national average increase of $13 \%$ to $16 \%$. Although, these rates are comparable with other Atlantic Provines, NL remains well above the Canadian average having the highest prevalence of adult obesity (Table 3.4$).^{27}$ 
Table 3.4 The Prevalence of Adult Obesity in Canada by province, based on self reported heights and weights 1995-2005

\begin{tabular}{|c|c|c|c|c|}
\hline & \multicolumn{2}{|c|}{$1995 / 96$} & \multicolumn{2}{|c|}{2005} \\
\hline & Preval & o $\quad(95 \%$ CI $)$ & Prevalence \% & $(95 \% \mathrm{Cl})$ \\
\hline Canada & 12.7 & $(12.0,13.3)$ & 15.5 & $(15.1,15.8)$ \\
\hline $\mathrm{BC}$ & 10.2 & $(8.5,11.8)$ & 13.2 & $(12.4,14.0)$ \\
\hline Quebec & 11.1 & $(9.6,12.5)$ & 14.2 & $(13.5,14.8)$ \\
\hline Alberta & 12.0 & $(9.7,14.2)$ & 15.8 & $(14.8,16.8)$ \\
\hline Ontario & 13.3 & $(12.2,14.5)$ & 15.1 & $(14.5,15.6)$ \\
\hline Manitoba & 14.9 & $(12.0,17.8)$ & 18.1 & $(16.8,19.4)$ \\
\hline PEl & 15.4 & $(12.7,18.1)$ & 22.5 & $(19.9,25.2)$ \\
\hline SK & 16.4 & $(13.6,19.2)$ & 20.6 & $(19.2,22.0)$ \\
\hline Nova Scotia & 16.7 & $(14.1,19.2)$ & 20.7 & $(19.1,22.3)$ \\
\hline New Brunswick & 17.2 & $(14.1,20.3)$ & 22.5 & $(20.8,24.2)$ \\
\hline Newfoundland and Labrador & 16.5 & $(13.5,19.4)$ & 23.8 & $(21.9,25.7)$ \\
\hline
\end{tabular}




\subsection{Actiology of Obesity}

Obesity is a result of an imbalance of energy intake and energy expenditure. 167 The actiology of most human obesity is unknown. ${ }^{168}$ There has been a marked increase in the prevalence of obesity over the last 25 years, suggesting alternations in various environmental factors. Research suggests that genetics also plays a role in some forms of obesity. Body size depends on the complex interaction between genetic background and environmental factors.

\subsubsection{Environmental Factors}

The increase in the prevalence of obesity over a relatively short period of time points to the changing environment such as an increase in sedentary occupations and leisure activities, availability of technology, increased availability of fast food and

processed food found in supermarkets as some of the factors responsible. ${ }^{169-170}$

The influence of environmental factors on body weight has been reported in a number of studies. However, the majority of these studies focus on aboriginal people who have moved from a traditional way of life to an urban lifestyle. The change in the lifestyle has had a dramatic influence on the health of the Pima Indians. These Indians moved from the Sierra Mountains in Mexico to Arizona. The change of lifestyle to an urban lifestyle has led to an epidemic of obesity and diabetes. The diet of the urban Pimas is much higher in fat $(50 \%$ of energy intake) than their traditional diet ( $15 \%$ of energy as fat) and their lives are more sedentary compared to the Pimas who remained in the mountains and who have kept to a traditional diet. Traditionally, the Pimas are physically active as farmers, sawmill workers and have a much lower incidenec of obesity and 
diabetes as compared to their Arizona kindred. ${ }^{171}$ The aboriginals in northern Australia are another high-risk population whose weight and health status has been compromised by exposure to a change in the environment. Urbanized aboriginals are heavier than their lean hunter-gatherer kindred and have a higher prevalence of type II diabetes. ${ }^{172}$

In Canada as well, a high prevalence of obesity and its associated chronic diseases are reported throughout aboriginal communities. ${ }^{173-175}$ Obesity and diabetes were undiagnosed or unknown in Aboriginal populations in Canada before the 1950's. However there has been an increase in both of these conditions reported in Aboriginal peoples. ${ }^{173-175}$ Much of the information on the impact of changing environments comes from aboriginal populations who have changed their lifestyle dramatically from a more traditional way of life to a more modern urban lifestyle. Although, changing environments play a role in the recent obesity epidemic, behavioral factors such as diet, physical activity and socioeconomic status are also very important.

\subsubsection{Diet and Physical Activity}

A change in body weight is caused by either a change in the amount of energy consumed, the amount of energy being expended or by changes in both. The following equation illustrates this relationship:

$$
\text { Energy }_{\text {in }}-\text { Energy }_{\text {out }}=\Delta \text { Body }_{\text {Weight }}{ }^{176}
$$

Routine surveillance data on nutrition is limited in many countries. Health and nutrition surveys collecting data on eating habits have shown that over the past few decades when overweight and obesity were increasing, the energy intake through diet was maintained or 
decreased. ${ }^{177}$ Data on the consumption of foods is reported using either surveys on nutrition or statistics on the disappearance of food items. The findings from these reports are not always consistent.

Published data available in Canada supports the theory that there has been a change in Energy out $_{\text {which }}$ has led to the recent increase in body weight. During the last 30) years, several surveys on nutrition have been administered to the population in Canada. In the carly 1970's, a national nutritional survey was conducted which was representative of the Canadian population and this survey was repeated in 1997. During the 1990's many provinces conducted their own provincial nutritional surveys. These surveys gathered specific and very detailed information from the adult population on food intake..$^{178-181}$

These surveys suggest that in the late 1990's the energy intake and total fat consumption of the Canadian population was lower when compared to the surveys conducted 30 years previously. These findings were comparable with the findings from surveys on nutrition from both the UK and the USA noting a decline in overall energy intake since the $19600^{\circ}$ s. ${ }^{170.182}$

These findings are based primarily on nutrition surveys and self reported intake of food. This empirical evidence suggests that using the formula for $\Delta$ Body Weight, it is more likely to be a decrease in Energy out rather than an increase Energy in $_{\text {in }}$ that aceounts for the recent increase in body weight.

Another way to examine trends in eating habits or Energy in. is to analyze the disappearance of foods. A report on the disappearance of Food Statistics reported by 
Statistics Canada in 2002, provided evidence in contrast to the above findings based on nutrition surveys. ${ }^{183}$ According to this report, the total amount of energy consumed per capita per day increased by over $18 \%$ between 1991 and 2002. These findings highlight an increase in the consumption of fat, as the main cause for the overall increase in body weight. The report suggested the proportion of energy consumed from carbohydrates and the proportion of protein consumed remained consistent when compared to ten years ago.

A report on the nutrition of the NL population supports the findings that there has been an increase in Energy $\mathbf{y i n}_{\text {in }}$. A diet consisting of more energy or calories was being consumed than necessary to meet the recommended daily intake i.e. following Canada"s Food Guide. A larger proportion of an individual's Energy in was attributed to fat intake. while less energy was attributed to carbohydrate intake. ${ }^{184}$

There are inconsistencies in these findings. One body of evidence provides support for the theory that Energy $y_{\text {in }}$ has decreased, while the second body of evidence provides information suggesting that Energy in $_{\text {in }}$ has increased and is mainly duc to an increase in the pereentage of fat consumed. It is difficult to determine the extent to which Energy $y_{\text {in }}$ has been either increasing or decreasing, due to the lack of longitudinal surveillance data on dietary habits.

Different methodologies used to assess energy intake may be a possible explanation for the inconsistent findings. While health surveys are generally reliable and valid in their collection of some variables such as demographics, health status and morbidity, they may not be as reliable when assessing the accuracy of food intake. This may be due to recall bias and to the fact that individuals may not remember what they 
have eaten over a specific time period. Another factor to consider is social desirability bias. The individual provides responses that the interviewer wants to hear. Consequently, it is possible when individuals respond to questions on food intake; the frequency of eating certain foods is inadvertently underestimated.

Other researchers have suggested that the most recent increase in obesity is due to a decrease in Energy Eut $_{\text {, and not due to an increase in Energy }}$ in through diet. A decrease in activity during work, leisure time and extra-curricula pursuits has been documentcd. ${ }^{176,182,185-186}$

Research suggests that regular physical activity protects against overweight, obesity and chronic disease, ${ }^{21}$ however the individual-level of physical activity and or exercise is not the main component of Energy ${ }_{\text {out }}{ }^{176}$ One graphical representation of energy expenditure is found in Figure 3.2. Total energy expenditure (TEE) is the sum of three components: resting energy expenditure (REE) and the thermic effect of food (TEF) and physical activity-related energy expenditure (PAEE). ${ }^{176}$ Figure 3.2 illustrates how total energy expenditure (TEE) is broken down into components and compares active and sedentary individuals. 


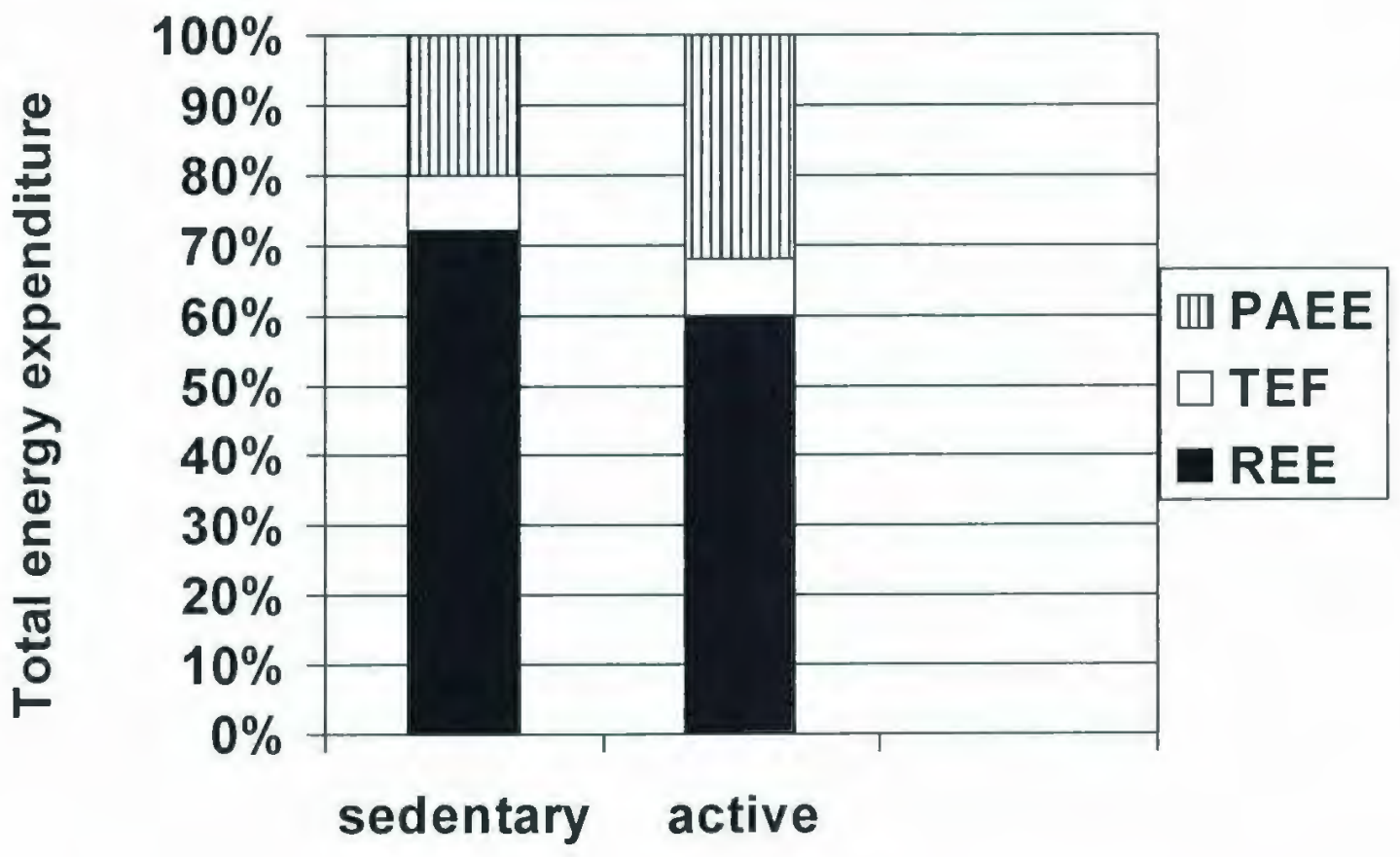

Figure 3.2 Components of Total Energy Expenditure (TEE) in Sedentary (1800 kcal/day) and Active Persons (2200 kcal/day)

Total energy expenditure (TEE) = Resting energy expenditure (REE) + Thermic effect of food (TEF) + Physical activity energy expenditure (PAEE) 
The majority of human energy expenditure is consumed through the body's metabolism at rest. Resting energy expenditure comprises on average $60-80 \%$ of the total energy expenditure in most people. Resting energy expenditure is the energy required by the body to maintain basic functions such as pumping blood, synthesizing hormones, and maintaining body temperature. The PAEE is the component of energy expenditure that is most under voluntary control, because it is influenced by the amount of physical activity performed. This component is the most variable component of energy expenditure and can range from $10 \%$ of TEE in sedentary individuals up to $40 \%$ of TEE in highly active individuals. Total energy expenditure includes both voluntary and non voluntary actions. Voluntary actions include daily activities and non voluntary actions include basic metabolic needs. Physical activity energy expenditure provides the greatest source of flexibility in the energy expenditure system, and it is through this component that large changes in energy expenditure can be achieved. The thermic effect of food refers to the increase in energy expenditure associated with digestion, absorption and storage of macronutrients. On average, the thermic effect of food makes up on average $8 \%$ of total expenditure out.

Figure 3.2 demonstrates that an active person has control over on average $35 \%$ of TEE compared to a sedentary person with control over only $20 \%$ of TEE. The largest component of Energy out is made up of the REE with the addition of the TEF. Increasing physical activity on its own is unlikely to decrease body weight. 


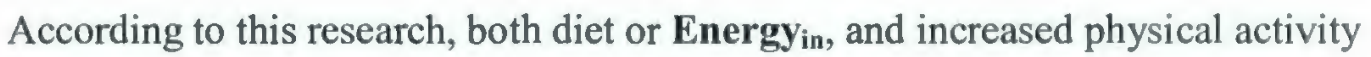

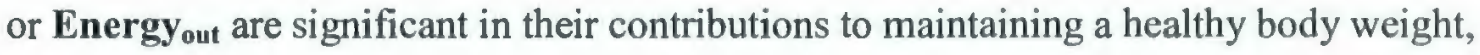
the normal BMI range associated with least health risk.

\subsubsection{Socioeconomic Factors}

Socioeconomic status (SES) is conceptualized as a combination of financial, educational and occupational influences. ${ }^{137}$ Socioeconomic factors such as low education and loss of employment appear to be correlated with obesity. ${ }^{169,}{ }^{187}$ In an early crosssectional review, 144 published studies were reviewed and the relationship between SES

and the level of obesity was examined. ${ }^{188}$ The authors found a strong inverse relationship between obesity and SES among women in industrialized countries, such as the USA and Western Europe. In most countries, however when these studies were published, the generally accepted cut-off for defining obesity was a $\mathrm{BMI} \geq 27$, in contrast to the present day internationally accepted cut-off of a $\mathrm{BMI} \geq 30$.

A more recent review identified 34 longitudinal studies from developed countries which assessed the relationship of various measures of SES with weight change in adults. ${ }^{189}$ For non-black samples, SES was assessed using occupation only. There were consistent inverse associations between occupations and weight gain for men and women. When the level of SES was assessed using education, the evidence was slightly less consistent, but provided some support for the inverse relationship between SES and weight change. However, the findings were inconsistent, when income was included as an indicator of socioeconomic status. When referring to the association between SES and obesity, only one component occupation was consistently inversely related to increasing 
weight change. The relationship between body weight and socioeconomic status was less consistent among men and children.

\subsection{The Genetics and Medical Causes of Obesity}

Several rare genetic causes of obesity have been described. These include syndromes such as Prader-Willi and Bardet Biedl syndrome where obesity is just one of many features. ${ }^{190}$

Recently, a strain of mice (i.e., the ob/ob mouse) were identified and the aetiology of this obesity was found to be related to a defect in the gene known as the ob gene. The ob gene encodes a protein leptin which is produced in adipose tissue. Leptin appears to act as a satiety factor. Mice with mutations with the ob gene become obese due to hyperphagic (overeating). This gene is present in humans and the leptin protein is found in human adipose tissue. Humans with mutations with the ob gene have been identified, however, to date this accounts for a very small percentage of the overall obese population. ${ }^{191}$

In addition, endocrine disorders due to a hormonal imbalance can cause weight gain. ${ }^{192}$ Cushing's syndrome with a predominance of central fat distribution is the most common endocrinological cause of obesity. This syndrome is caused by an excess production of cortisol. Hypothyroidism, another endocrine condition may be a contributing factor of excessive weight gain. ${ }^{190}$ Drug-induced weight gain is also common in individuals taking antipsychotics, steroids and insulin. ${ }^{193}$

Due to the rare nature of purely genetic and medical causes of obesity, it appears that for most people, the genetic predisposition will not be defined by one gene but by 
multiple genes and their interaction with the environment. ${ }^{168}$ Attempts are on-going to identify these multiple genes. In large population surveys, more than 244 genes, markers and chromosomal regions have been linked to human obesity. ${ }^{195}$ Adoption studies demonstrated that genes exerted a strong effect on body weight. The weight of adults who were adopted as children, related more closely to that of their natural parent, and not to the new or adoptive parent. ${ }^{196}$ In addition, it was shown that twins who were separated and raised in different environments, demonstrated a similar heritability or increased likelihood of passing on the trait for overweight and obesity. ${ }^{197}$ In another study of twins, identical male adult twins were fed a surplus of $1000 \mathrm{keal}$ per day, six days a week for a period of 100 days. Significant increases in body weight and fat mass were observed in these adults, following the period of overfeeding. The findings demonstrated that there were considerable differences between the pairs of twins and how they responded to excess calories. There was at least three times more variance in response to overfecting between pairs of twins than within pairs of twins, for the gain in body weight, fat mass and fat-free mass. This study demonstrated that some individuals were more at risk than others for gaining weight when energy intake surplus is the same for everyone, and when all subjects were restricted to a sedentary lifestyle. The 'within' identical twin pair response (i.e., similar weight gain) suggests that the amount of fat stored is influenced by genotype. $^{198}$

In families with one or two morbidly obese parents, the risk of an offspring becoming obese is two to three times that of those having nomal weight parents, suggesting that both genetics and the environment play a role in determining the 
likelihood of an individual's weight status. ${ }^{199}$ A better understanding of the complex aetiology of obesity may lead to more effective means in treating and preventing obesity in the population. 


\section{Chapter 4 Methods}

\subsection{Introduction}

The purpose of this chapter is to provide a detailed discussion of the methods used to examine the relationship between level of BMI as a reflection of obesity and health services utilization and associated costs, for a representative sample of the province of NL, Canada using individual-level data from the 2001 CCHS. This chapter provides an overview of the (i) study design (ii) study population/sample (iii) database linkage procedure (iv) variables analyzed in the eurrent study (v) data analysis and (vi) ethical considerations.

\subsection{Study Design}

This is a population-based study of adults aged between 20 and 64 years of ange living in NL. The population was stratified by BMI category for the purpose of the analysis. The study included: (i) a secondary analysis of a sub sample of data from a National cross-sectional health survey and (ii) an analysis of actual health services utilization and direct costs over a five-year period. Information on factors associated with obesity/morbid obesity and subjective health services utilization were obtained from the CCHS. Objective health services utilization was obtained through linkage of the CCHS to two administrative databases; the Clinical Database Management System (CDMS), the provincial hospital separations database and the Medical ('are Program (MCP) database which processes claims for fee-for-service (FFS) physicians in the province. 
The data on subjective health services utilization referred to the 12 months prior to the survey date, while the objective health services utilization data was examined over a five-year period from January 1998 to December 2002 for physician utilization and from September 1999 to August 2003 for hospitalization utilization. This included utilization data on the period 30 months before and 30 months after an individual's survey date. The timeframe for the study (i.e., five years) was limited by the availability of the data. A time lag existed between collection of the data, analysis by CIHI, standardization by the Provincial Department of Health and Community Services and the distribution of data to the provincial health agencies.

\subsection{Study Population/Sample}

The 2000/2001 CCHS was conducted over a 12 month period. This survey was administered to 131,535 Canadians through personal interviews either by telephone or inperson. The response rate was $84.7 \%$. The survey used a multi-stage cluster sampling design to collect information related to the health of non-institutionalized individuals and included household residents aged 12 years of age and over in all provinces and territories. Indian Reservations, Canadian Forces Bases, and some remote areas were not included. The total sample size was sufficient to provide reliable cross-sectional estimates at the provincial and sub-provincial or health region level for some variables.

The current study included analysis of survey responses exclusively on the NL portion of Statistics Canada's 2000/2001 CCHS. Key inclusion criteria for the current study included: agreement to share survey responses with Health Canada and other health information agencies and permission to link individual survey responses with other 
provincial health information databases. Individuals had to be between 20 and 64 years of age and have a calculated BMI available. The age range was limited. Rescarch suggests BMI may not be an accurate predictor of excess body fat or associated health risk in older age groups as both men and women naturally lose lean body mass through the life cycle. For example, although the percentage of body fat increases with age, BMI can remain constant. ${ }^{20}$ As a result; researchers limit the use of BMI to this middle age-group. Pregnant women were also excluded from this study.

In this study, BMI, detined as weight in kilograms divided by height in metres squared $\left(\mathrm{kg} / \mathrm{m}^{2}\right)$ was classified using the system endorsed by both the World Health Organization and Health Canada. This classification system was presented in Table 3.1. Underweight, overweight and all obese BMI categories are associated with increased health risk. ${ }^{17,21}$ As described earlier in this study, a BMI $\geq 30$ and $<35 \mathrm{~kg} / \mathrm{m}^{2}$ will be considered obese. Obese class II (BMI $\geq 35)$ and obese class III (BMI $>40)$ will be combined and described as morbid obesity $\left(B M I \geq 35 \mathrm{~kg} / \mathrm{m}^{2}\right)$.

The total eligible study population was 3734 survey respondents in NL. All survey respondents consented to share their non-identifiable survey responses with Health Canada and other health information agencies, and 94\% (11-3497) consented to the linkage of their individual health survey responses with other provincial health information. In total, one thousand and seventy-five $(n=1075)$ individuals were excluded from the study. Individuals excluded were those under 20 or over 64 years of age and 31 pregnant women. In addition 25 individuals had invalid or missing information on heights and/or weights. The study sample was reduced to 2366 individuals. Caltegorizing 
the study population into BMI groupings resulted in the following: 88 I normal weight; 916 overweight; 407 obese, I 41 morbidly obese and 21 underweight individuals. The underweight population of 21 individuals was excluded from further analysis due to the focus of the research hypothesis, that increasing levels of BMI are associated with an increased use of health services. The sample size for the secondary analysis of the health survey data was 2345 individuals, before the linkage. Figure 4.1 illustrates the ealculation of the final sample size before linkage. 


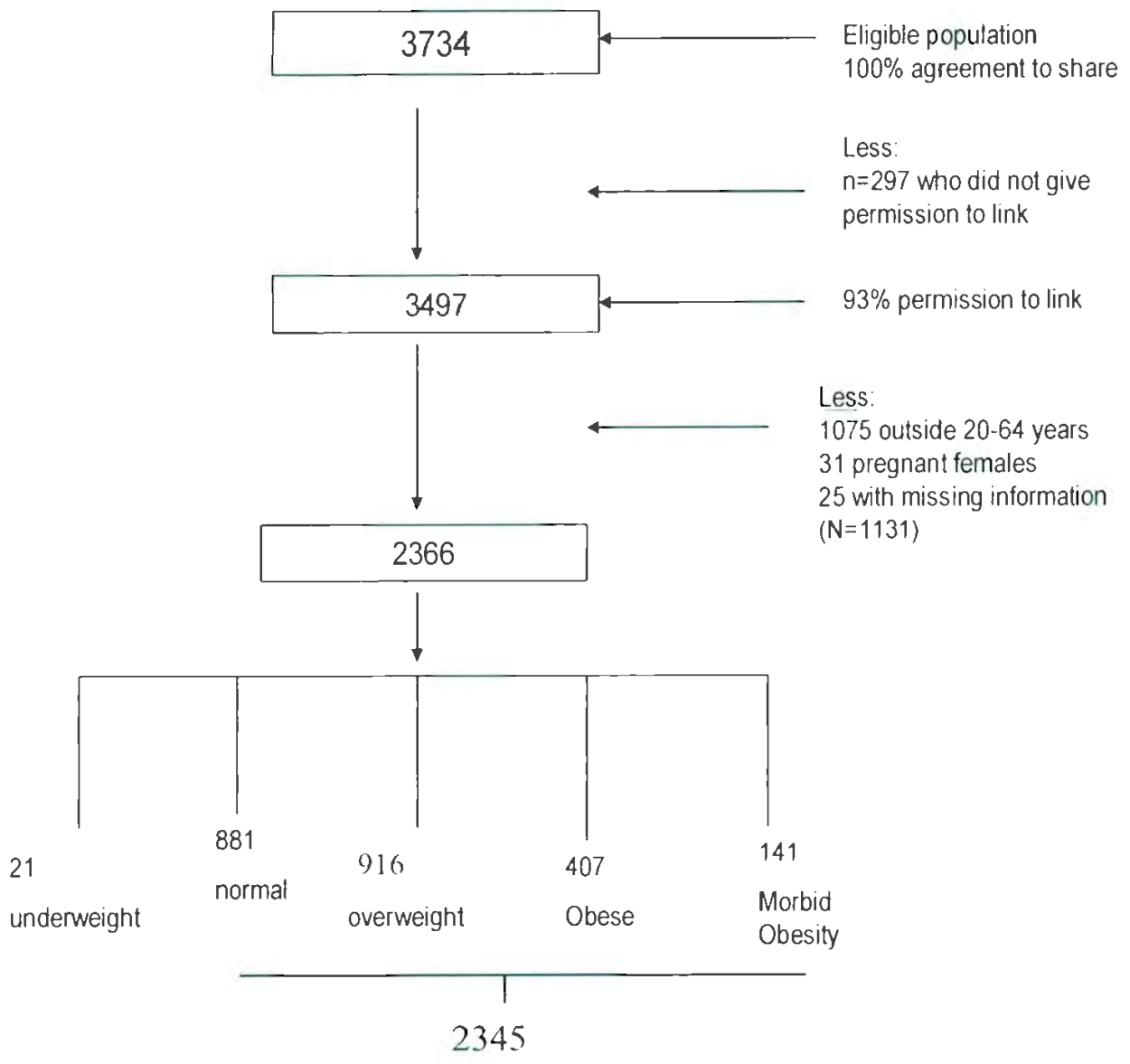

Figure 4.1 Flowchart of the Selection of Study Sample 


\subsection{Sources of Data}

The following section describes the data sources utilized for the research study, the linkage process and the variables of interest under study. The research study used three data sources: (i) the NL component of Statistics Canada's 2000/2001 CCHS; (ii) the MCP database and (iii) the CDMS. The 2000/2001CCHS provided a sample representative of the population of NL with information on demographics, health status and lifestyle, chronic disease and subjective health services utilization while the health administrative databases provided an objective measure of health services utilization.

\subsubsection{The 2000/2001 Canadian Community Health Survey}

The Canadian Community Health Surveys were developed by Statistics Canada to address gaps in health information at the regional or sub-provincial level and to explore the health issues and the healthcare concerns of Canadians. The survey commenced in 2000 and is repeated every two years. The primary objective of the CCHS is to provide timely cross-sectional estimates across provinces on health determinants, health status and health system utilization. Survey results provide a "snap-shot" of the health status of the residents in a province or territory. The CCHS provides health information for governments, health boards, health agencies researchers and communities contributing to health policy, research and the development of programs.

The CCHS takes approximately 45 minutes to complete: thirty minutes of "common content" questions being asked to all sample units in all provinces in order to meet the basic health data requirements on an on-going basis. As well, ten minutes of questions on "optional content" are offered to the provinces. These optional content 
questions are in modules and offered to the provinces at an additional cost. Individual provinces can decide if they want to purchase the "optional content" questions depending on their individual needs for information. An example of a topic for optional content questions is smoking cessation. Standard questions on socio-economic and demographic characteristics complete the interview. The survey questionnaire and comprehensive information is available on the Statistics Canada website, ${ }^{20(1-202}$ The relevant survey questions used in the current study on health services utilization, health status and lifestyle included:

- Do you have a regular medical doctor?

- In the past 12 months, have you been an overnight patient in a hospital, nursing home or convalescent home?

For how many nights?

- Not counting when you were an overnight patient, in the past 12 months, how many times have you seen or talked on the telephone about your physical, emotional or mental health with a family doctor or general practitioner, a mental health provider or another type of doctor (e.g., a specialist such as a surgeon)?

- In the past 12 months have you received any hospital services at a hospital either as an inpatient an outpatient or an emergency room patient?

- In the past 12 months, not counting hospital visits, have you received any health care services from a family doctor or other physician?

- How tall are you without shoes on? 
- How much do you weigh?

- In general would you say your health is excellent? Very good? Good'? Fair or Poor?

- Now I'd like to ask you about certain chronic health conditions which you may have. We are interested in long-term conditions that have lasted or are expected to last 6 months or more and that have been diagnosed by a health professional.

- Questions included: Do you have food allergies? Other allergies"? Asthma? Arthritis? Back problems? High blood pressure? Diabetes? Cancer (what type?) Interviewer lists 25 chronic conditions.

- For certain groups of questions, derived variables were calculated using validated formulas. Derived variables combine answers to several questions to form one composite variable with an associated value. Questions on level of income, education, physical activity, fruit and vegetable consumption, alcohol intake and smoking behavior were combined into derived variables.

\subsubsection{Access to Survey Data}

Statisties Canada stores and maintains its survey datal on several files two of which include: a "master file" and a "share" file. In addition a "link file" can be aceessed in order to link individual survey responses from either the "master" or the "share" file with other individual health information. 
The "master file" includes survey responses and identifying information such as names, addresses and telephone numbers for all respondents. The master file is not available to be taken off-site for research purposes. The master file will include survey responses for individuals who have not agreed to share responses. If a researcher(s) can show that access to the 'master file' is required for research purposes and the other files do not provide the needed information, access can be provided through a remote access service or through the Research Data Centres Program. Researchers are provided with 'dummy data' on a $\mathrm{CD}$ rom and are able to submit research questions and required analysis by email to Statistics Canada. Analysis on the master file is performed by an employee of Statistics Canada and the results returned to the researcher.

Statistics Canada provides a national "share file" to each provincial ministry of health and other "share' partners. The "share file" is stripped of all personal identifiers and is available for research purposes. This file contains survey responses and information on any respondent who has agreed to share their responses with other jurisdictions. In addition, for those survey respondents who agree to allow the linkage of their survey responses to other health information agencies through the provision of their health insurance number a "link file" is produced and provided to provincial ministries of health.

The link file contains a unique identifier, the age, sex, date of birth and the health insurance number for each survey respondent. The 'link' file is housed at the provincial Department of Health and Community Services. The NL Centre for Health Information has an agreement with both the Provincial Department of Health and Community 
Services and Statisties Canada to access this file. The present study utilizes both the "share" and "link" files.

\subsubsection{Medical Care Plan Database}

The Medical Care Plan (MCP) is a comprehensive system of public medical care insurance covering the cost of fee-for-service (FFS) physician services for eligible residents of the province of NL. The MCP database is housed within the Provincial Department of Health and Community Services. The MCP database captures information on sex and age of patient, diagnosis, provider's specialty (e.g., general practitioner or other specialty), service date (including year), fee code and province of beneticiary. The MCP fee code describes all potential fee grouping categories: office consultations, home consultations, in-patient consultations, outpatient and emergency visits, diagnostic and therapeutic procedures, in-hospital diagnostic procedures, radiology and surgical procedures. In all cases, only visits were included where the physician was colled as the primary provider (exeluding code for surgical assistant and anesthetist). Fee codes range from 00 I (general practitioner) to 082 (medical officer of health), although there are some codes not presently used (c.g., NC or no code). Codes are set by the Medical Care Plan Board (Table 4. 1). The completeness of the physicians" claims database has not been verified, however as the information collected is an integral part of the claims for payment it is considered to be complete in terms of numbers of visits and type of physician visited (c.g., family doctor versus specialist). Access to this database was provided to the researcher through the NL Centre for Health Information. 
Table 4.1 FFS Specialty codes and Specialty description

\begin{tabular}{|c|c|c|c|}
\hline 001 & GENERAL PRACTICE & 048 & PAEDIATRIC ONCOLOGIST \\
\hline 002 & ANAESTHETIST & 049 & PAEDIATRIC NEPHROLOGIST \\
\hline 003 & NC- code not currently used & 050 & PAEDIATRIC IMMUNOLOGIST \\
\hline 004 & EMERGENCY MEDICINE SPECIALIST & 051 & PAEDIATRIC HAEMATOLOGIST \\
\hline 005 & NC - code not currently used & 052 & NEONATOLOGIST/PERINATOLOGIST \\
\hline 006 & DERMATOLOGIST & 053 & PHYSICAL MEDICINE SPECIALIST \\
\hline 007 & NC- code not currently used & 054 & NC- code not currently used \\
\hline 008 & GENERAL SURGEON & 055 & PLASTIC SURGEON \\
\hline 009 & NC- code not currently used & 056 & NC- code not currently used \\
\hline 010 & CARDIAC SURGEON & 057 & PSYCHIATRIST \\
\hline 011 & VASCULAR SURGEON & 058 & NC- code not currently used \\
\hline 012 & THORACIC SURGEON & 059 & UROLOGIST \\
\hline 013 & INTERNIST & 060 & NC- code not currently used \\
\hline 014 & NC- code not currently used & 061 & DENTIST \\
\hline 015 & CARDIOLOGIST & 062 & ORAL SURGEON \\
\hline 016 & ENDOCRINOLOGIST & 063 & ORTHODONTIST \\
\hline 017 & RESPIROLOGIST & 064 & PERIODONTIST \\
\hline 018 & RHEUMATOLOGIST & 065 & PEDODONTIST \\
\hline 019 & GASTROENTEROLOGIST & 066 & DENTURIST \\
\hline 020 & MEDICAL ONCOLOGIST & 067 & PATHOLOGIST \\
\hline 021 & NEPHROLOGIST & 068 & NC- code not currently used \\
\hline 022 & CLINICAL IMMUNOLOGIST & 069 & DIAGNOSTIC RADIOLOGIST \\
\hline 023 & HAEMATOLOGIST & 070 & NC- code not currently used \\
\hline 024 & GERIATRIC MEDICINE SPECIALIST & 071 & OPTOMETRIST \\
\hline 025 & MEDICAL GENETICS SPECIALIST & 072 & NON-CERTIFIED ORAL SURGEON \\
\hline 026 & NUCLEAR MEDICINE SPECIALIST & 073 & PUBLIC HEALTH DENTAL \\
\hline 027 & INFECTIOUS DISEASE SPECIALIST & 074 & DEVELOPMENTAL NEUROLOGY \\
\hline 028 & NEUROLOGIST & 075 & DEVELOPMENTAL PEDIATRICIANS \\
\hline 029 & NC- code not currently used & 076 & ENDODONTIST \\
\hline 030 & NEUROSURGEON & 077 & RADIATION ONCOLOGIST \\
\hline 031 & NC- code not currently used & 078 & NC- code not currently used \\
\hline 032 & OBSTETRICIAN/GYNECOLOGIST & 080 & PEDIATRIC SURGEON \\
\hline 033 & NC- code not currently used & 081 & PAEDIATRIC INTENSIVIST \\
\hline 034 & GYNECOLOGY ONCOLOGIST & 082 & MEDICAL OFFICER OF HEALTH \\
\hline 035 & OPHTHALMOLOGIST & & \\
\hline 036 & NC- code not currently used & & \\
\hline 037 & ORTHOPAEDIC SURGEON & & \\
\hline 038 & NC- code not currently used & & \\
\hline 039 & OTOLARYNGOLOGIST & & \\
\hline 040 & NC- code not currently used & & \\
\hline 041 & PAEDIATRICIAN & & \\
\hline 042 & NC- code not currently used & & \\
\hline 043 & PAEDIATRIC CARDIOLOGIST & & \\
\hline 044 & PAEDIATRIC ENDOCRINOLOGIST & & \\
\hline 045 & PAEDIATRIC RESPIROLOGIST & & \\
\hline
\end{tabular}




\subsubsection{Clinical Database Management System}

All provincially administered healthcare facilities in NL submit hospital discharge data to the Canadian Institute for Health Information or CIHI, which describes inpatient and surgical day care services. The data are reviewed for data quality and specific values are calculated (e.g., resource-intensity-weight, case mix groupings and level of complexity index) and added to the database by CIHI before the discharge abstract database (DAD) is provided to the provincial Department of Health and Community Services where it is housed. Subsequently data is provided to the NL Centre for Health Information where the quality of the information is verified. In addition, the NL Centre for Health Information ensures standardization of the data prior to integrating the data into the provincial database, the CDMS. This database includes all discharge diagnostic codes based on the International Classification of Diseases (ICD).

The ICD provides the rules for coding and classifying cause-of-death data. Diseases listed on death certificates are assigned specific codes according to this system. The ICD was developed collaboratively between the WHO and ten international centers. The system allows mortality data to be collected and compared among different populations and countries. For example, the list of ICD covering endocrine, nutrition and metabolic diseases as well as immunity disorders includes diseases coded from 240 to 279.

The CDMS contains demographic, clinical and procedural data on all in-patient and surgical day care patients. This database includes information on residents of NL receiving care in all provincial hospitals as well as information on NL residents receiving 
care outside the province. Access to this database was provided through the NL Centre for Health Information.

\subsection{Database Linkage Procedure}

As part of the health survey, respondents indicated their willingness to share and/or link their individual survey responses with other jurisdictions and/or to other provincial health administrative data. Respondents who agreed to share and link their survey responses provided their health insurance MCP numbers. MCP numbers were used to link the health survey sample to the MCP claims file and the CDMS in order to obtain physician and hospital health services utilization. A detailed description of the linkage process can be found in Appendix B.

\subsection{Measures in the Current Study}

The following section describes the dependent and independent variables of interest.

\subsubsection{Dependent Variables}

The primary dependent variables of interest were 1) self reported presence of chronic disease 2) measures of objective health services utilization and 3) associated direct costs over a five-year period from 1998-2003. Using Andersen`s Model, the measures of health services utilization are described as Health Behaviors (see Table 4.2). The specific measures of health services utilization included visits to a physician as well as measures of hospitalization. Direct costs included total costs of physician services and total inpatient costs. 
To examine the relationship between level of BMI and self reported chronic diseases, the dependent variable was the presence of a chronic disease as diagnosed by a health professional $(1=$ yes, $2=$ no). The variables describing self reported health services utilization are listed in Appendix C Table 1a and the variables describing the presence of chronic diseases were extracted from questions asked in the 2000/2001 CCHS and are listed in Appendix C Table $1 \mathrm{~b}$.

The objective data on health services utilization was obtained through individual record linkage to the MCP and CDMS databases. The data extracted from the MCP physician claims database of particular relevance to the current study included: provider's specialty (e.g., general practitioner or other specialty), fee code (i.e., $0=\mathrm{GP}$, all else = specialist). Service date and service year were also collected. To examine the relationship between level of BMI and the measures of objective health services utilization, five regression models were developed where the measures of utilization were designated as the outcome variables and the level of BMI along with the other covariates were designated the independent variables.

In the first model the dependent variable was the sum of the number of visits to a GP. In the second model the dependent variable was sum of the number of visits to a specialist doctor. For each of these dependent variables all visits were summed over the study period to obtain the total number of visits per individual. In the third, fourth and fifth models, the dependent variables were measures of hospital use. Hospital data was abstracted from the CDMS and included information on: episode type (e.g., $0=$ acute and l=surgical day care); length of stay or LOS; Resource Intensity Weight (RIW) and Day 
Procedure Group weight (DPG_W). These two quantitative variables (i.e., RIW, DPG_W) measure resources used by in-patients or surgical daycare patients and are calculated using a methodology developed by the Canadian Institute for Health Information (CIHI). ${ }^{203}$ The rationale for developing this methodology is that patients have varying levels of illness severity and require different levels of healthcare resources. The Canadian Institute for Health Information has developed a methodology for costing hospital services based on an individual's use of hospital resources. These variables combine resources used with published patient-specific cost data in order to determine a specific value. These resource-intensity-values provide a key element in developing the approach of combining clinical and financial data in utilization management. This approach is based on a relative resource allocation methodology for estimating a hospital's inpatient-specific costs for both acute and day care. Resource-intensity-weights are used to standardize hospital in-patient case volumes across the country. The RIW and DPG_W values can be used to estimate patient-specific resource use and these variables can then be combined with cost data and used to estimate hospital costs. Both these values are used to estimate the cost of a hospital stay either as an inpatient or day surgery patient as an outcome variable by multiplying the patient specific RIW's by the provincial cost per weighted case (CPWC) estimated and supplied by CIHI. A simple equation would be: Cost of hospital stay/procedure $=$ RIW/per patient X CPWC. The CPWC measures the average cost of treating inpatient acute care cases and is published annually in the CIHI Financial Performance Indicators report for all provinces. The CPWC for 2001 supplied by CIHI for the province of NL was $\$ 4512.00 .^{204}$ 
Specifically in the third model the dependent variable was the number of inpatient admissions and was obtained by summing the number of individual admissions. In the fourth model the dependent variable total length of stay (LOS). Length of stay was calculated by summing the total number of days per individual spent in hospital and in the fifth model, the dependent variable was the total number of day surgeries per individual.

As well to examine the relationship between level of BMI and direct costs, two more regression models were developed. In the first model the dependent variable was total physician costs associated with the services provided and the fee claimed as per the MCP database. In the second model the dependent variable was total costs for inpatient hospital services. Costs for day surgeries were not included in this analysis as there was not enough data to estimate day surgery costs. The coding of the DPG_W in the CDMS database commenced in February 2002 providing only 6 months of data on this variable for calculation purposes.

\subsubsection{Independent Variables}

\subsubsection{The Primary Relationship under Study}

The main independent variable of interest for the current study was level of BMI described as a categorical predisposing variable $(1=$ normal, $2=$ overweight, $3=$ obese, 4 $=$ morbidly obese). Confidence in using an individual's self reported BMI during the 2000/2001 CCHS as an estimate over the five-year period is supported by several published articles. ${ }^{205-209}$ These articles suggested adults who become overweight or obese are likely to remain in that weight category during their adult life and if any new change 
occurs in BMI, it tends to be in the direction of a higher weight category. Therefore based on these reports, the majority of individuals in the current study classified as obese at the time of the survey would probably have been classified as obese over the full five-year study period. However to provide evidence to this argument, a separate analysis was conducted using the longitudinal components of the National Population Health Surveys (Appendix D).

\subsubsection{Selection of Independent Variables from the Survey}

The following independent variables were extracted from the CCHS to represent those described in Andersen's model as Population Characteristics. Variables described as Predisposing in the model included age, sex, education level and marital status. The Enabling variables included access to a regular doctor, income level, rural/urban location and health region of residence. The Need variables included a measure of chronic disease (e.g., the presence and the number of chronic conditions) and the health status variables: physical activity, consumption of fruits and vegetables, alcohol consumption and smoking behavior as they reflect the health status or need of health services of an individual. There were no variables selected to represent the Environment or Outcomes and therefore were not included in Table 4.2. A detailed description of these variables can be found in Appendix C: Table $1 \mathrm{~b}$. 
Table 4.2 Independent and Dependent variables used in the primary analysis

\begin{tabular}{|c|c|c|c|}
\hline \multicolumn{3}{|c|}{ Population Characteristics } & \multirow[t]{2}{*}{$\begin{array}{l}\text { Health } \\
\text { Behavior }\end{array}$} \\
\hline Predisposing & Enabling & Need & \\
\hline age & regular doctor & chronic discase & $\begin{array}{l}\frac{\text { Physician }}{\text { visits }} \\
\text {-GP } \\
\text {-specialist }\end{array}$ \\
\hline $\operatorname{sex}$ & income level & & $\begin{array}{l}\text { Hospital use } \\
\text {-impatient } \\
\text { admissions } \\
\text {-LOS } \\
\text {-day surgeries }\end{array}$ \\
\hline education level & urban/rural location & & Direct costs \\
\hline marital status & $\begin{array}{l}\text { health region of } \\
\text { residence }\end{array}$ & $\begin{array}{l}\text { health status } \\
\text { level of physical } \\
\text { activity } \\
\text { consumption of } \\
\text { fruits \& } \\
\text { vegetables } \\
\text { alcohol/smoking } \\
\text { belavior }\end{array}$ & $\begin{array}{c}\text {-total } \\
\text { physician } \\
\text { costs } \\
\text {-total inpatient } \\
\text { costs }\end{array}$ \\
\hline
\end{tabular}




\subsection{Comparison of Subjective and Objective Visits to a Physician}

In the current study, both self reported and actual data on physician utilization was obtained. The self reported data was collected through the $2001 \mathrm{CCHS}$ for a period of 12 months prior to the survey and actual data was obtained through the linkage of the survey respondents to the physician claims database (MCP) over a 5-year period. Self reported data on physician utilization was collected though the use of two questions that asked survey respondents whether in the previous 12 months they had seen their regular or family doctor or 'another doctor'. These questions were able to pick up physician utilization, irregardless of whether the doctor was salaried or paid fee-for-service (FFS). The data on actual physician visits was limited to visits or services provided by physicians paid FFS, as this is the only information collected at a provincial level and is done so for payment purposes. In NL doctors on salary do not submit shadow or dummy invoices and therefore it is not possible to get information on visits made to salaried doctors.

The actual data provided many more data points per survey respondent. It was felt that for the purposes of the current thesis, in order to run the regression models developed to answer the research questions more data rather than less data would be beneficial (i.e., 5 years versus 12 months) as these extra data points would increase the ability to control for confounding variables.

According to the most recent report on the Distribution of physicians in NL (Annual Report I999/2000 Medical Care Plan), on average across the province, 66\% of all physicians are paid FFS. This percentage varies from a high of $77 \%$ in the Eastern 
Health Region to a low of $22 \%$ in the Northern Health Region. The proportion also varies within health regions. For example within the Eastern Health Region the proportion ranges from a high of $84 \%$ in St. John's, the capital city to a low of $42 \%$ in Clarenville while in the Northern Region the proportion ranges from a high of $77 \%$ in the Labrador City/ Wabush area to a low of $22 \%$ in St. Anthony Goose Bay area (Appendix E: Table 1).

Under the FFS coding scheme resources used or visits made can be categorized as office or home, in-patient, out-patient and emergency, diagnostic and therapeutic procedures, in-hospital diagnostic procedures, radiology and surgical procedures. Analysis of FFS physician visits over the 5-year period by code (e.g., office, home, radiology) demonstrated that $89 \%$ of GP visits were categorized as either office or home. Over the same time period only $15 \%$ of specialist services were classified as office or home visits while the majority ( $43 \%)$ were classified as radiology services followed by $12 \%$ and $9 \%$ categorized as in-hospital diagnostic procedures and out-patient and emergency visits respectively (Appendix E: Table 2).

Analyses to compare self reported and actual physician visits were stratified by BMI category and conducted on; the province as a whole, the four regional health boards and the urban and rural geographical regions. Using student t-tests, the average number of self reported visits to a GP and a specialist doctor were compared with actual FFS physician visits over a 12 month period. As the actual data included five years of data, for comparison purposes the total sum of FFS visits was divided by five for an annual sum 
and these numbers were compared with 12 months of survey data. These results are presented in Appendix E: Tables 3-4.

\subsection{Data Analysis}

Data analyses were conducted using the Statistical Package for Social Sciences (SPSS 13.5 for Windows) and the ' $\mathrm{R}$ ' software program in the Department of Statistics, Memorial University. Regression analyses using Statistics Canada health survey data were weighted due to the non-random sampling design of the survey. Statistics Canada uses a complex multi-stage design, with over-sampling for some sub-populations (i.e., health boards). Consequently, sampling weights are developed to account for survey design and to provide an adjustment for survey non-response. To account for sampling design the CCHS contains a "weight variable' which represents the number of individuals that each survey respondent represents. Statistics Canada suggests that all predictive analyses should be run incorporating the weight variable. ${ }^{213}$ Weighted analyses were not used for descriptive statistics; however for both the Poisson and multiple linear regression models, a sample weighting variable was employed.

\subsubsection{Descriptive Statistics}

Descriptive statistics were conducted to describe the characteristics of the sample studied stratified by level of BMI. As there were four groups of BMI (e.g., normal, overweight, obese and morbidly obese), the statistical test chi-squared was conducted on all categorical variables to determine the level of significance. Continuous variables such as the Health Utility Index and the number of disability days were analyzed using the Analysis of Variance (ANOVA). A preliminary analysis of the measures of health 
services utilization through histograms illustrated data that was positively skewed to the right and therefore not normally distributed (see Figure 4.2 for Total FFS visits).

Therefore reporting averages with standard deviations does not always clcarly illustrate the variability of the data. The outliers (data points that are greater or less than 3 standard deviations from the mean) can influence the average. The median as a descriptive measure of health service use ean be more accurate than the mean to describe the central tendency of the data and to compare the variability of the data. ${ }^{210}$ 


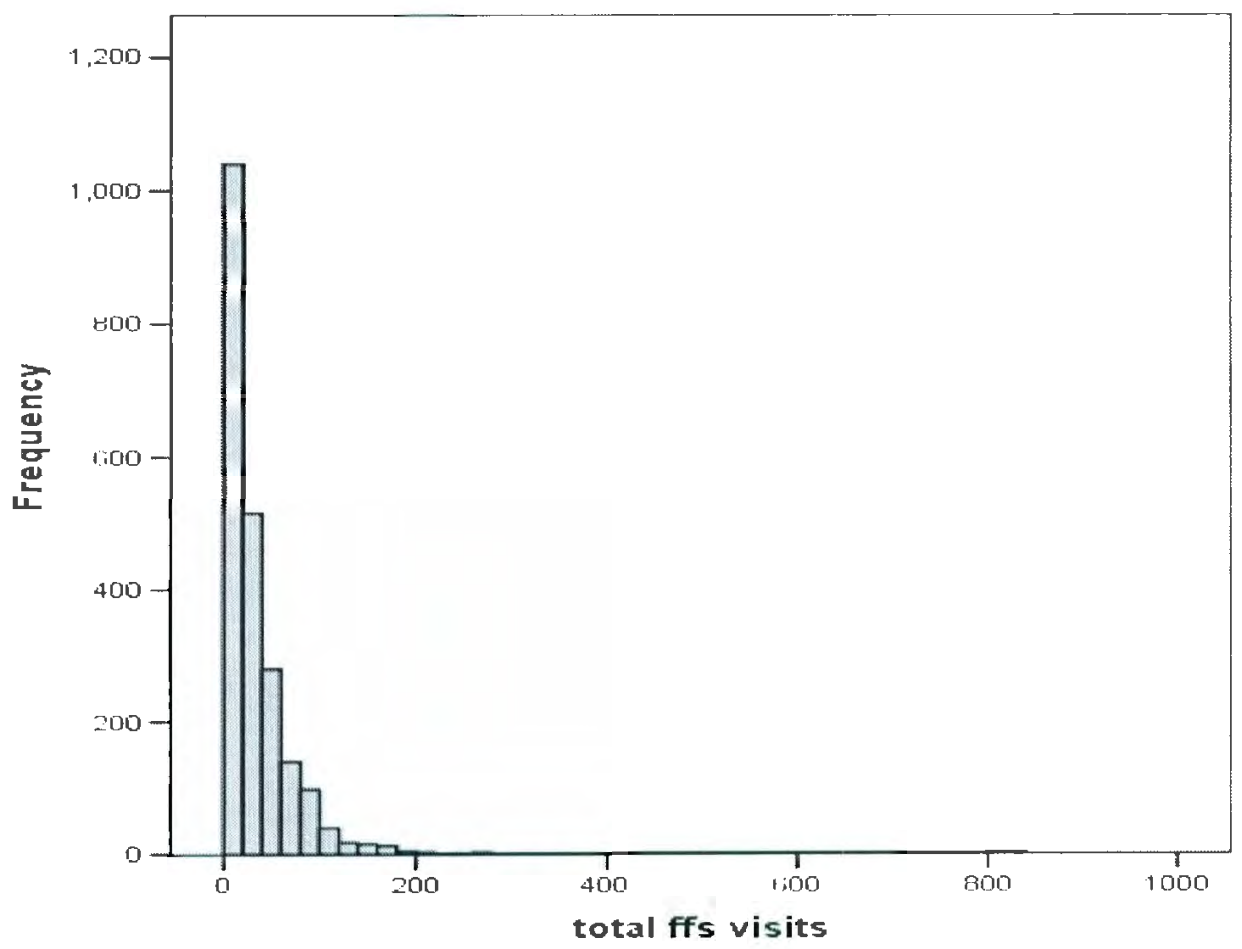

Figure 4.2 Total FFS visits 1998-2003 $(n=2177)$ 
In order to determine whether differences existed across level of BMI and the measures of HSU (e.g., number of visits to doctors, to hospitals and of nights spent in hospital) median tests were performed. To demonstrate the variability of the data, the

inter quartile range (i.e., $25^{\text {th }}-75^{\text {th }}$ percentiles) was reported. For categorical data, cross tabulations and chi-squared tests were conducted for gender, health region and smoking status. An alpha of .05 was selected as the significance level for tests of association and difference and all tests were two-tailed.

\subsubsection{Model Development}

The next section presents the inferential statistical models employed to estimate: i) the association between level of $\mathrm{BMI}$ and the presence of chronic disease, ii) the predictive relationship between level of BMI and health services utilization and iii) the predictive relationship between level of BMI and the direct costs associated with health services utilization.

Most statistical tests are based on a model whereby the response/dependent variable is a function of an independent variable(s). The classic equation format is $Y=f(x 1, x 2, x 3, x 4 \ldots)$ where $Y$ is the outcome variable and the $x$ 's are independent variables. This equation can take many forms and is dependent on the innate relationship with the variables. The most appropriate statistical model to fit any data set depends on the type of response variable. The association between $Y$ 's and X's determines the shape of the relationship of interest. For example to use a statistical test based on a linear model there must be a linear relationship (i.e., a straight line) between the dependent and independent variable (i.e., as $\mathrm{x}$ increases, $\mathrm{Y}$ increases at a constant slope). 
In the current study, several types of regression analyses were used to estimate the relationships under study. For variables where a linear relationship existed, the Ordinary Least Squares method (OLS) which minimizes the observed values of the response $Y$ and their mean per each unit of $\mathrm{x}$ was used. Where the response variable was a continuous numerical measure, as in the case of direct health system costs, multiple linear regression was employed. Logistic and Poisson regression models were developed where non-linear relationships existed. In contrast to the OLS methods, both these models used the Maximum Likelihood Estimation method (MLE), as the distribution of the response variables was binomial or Poisson. ${ }^{211}$

\subsubsection{Logistic Regression Models}

Logistic regression was used to determine whether the level of BMI was a predictor of the presence of chronic disease in this population controlling for age and gender. Given the binary nature of the dependent variable (e.g., presence of chronic disease $1=$ yes, $2=$ no), the choice of statistical test included both logit and probit techniques. Both techniques require the dependent variable to be dichotomous that is having only two outcomes, and therefore to be interpreted as probabilities. Under most circumstances, the conclusions will be similar for both logit and probit methods. The choice of test is primarily that of familiarity and one of preference and ease of interpretation. ${ }^{212}$ The advantage of using logistic regression is that the estimates of the coefficients in the equations can be interpreted easily as they are presented as odds ratios. Logistic regression was employed in this study. 
Logistic regression is part of a category of statistical models called generalized linear models or GLM and allows one to predict a discrete outcome, such as group membership from a set of independent variables that may be continuous, discrete or dichotomous such as presence/absence of a disease. The dependent variable is most often dichotomous, and can take the value of I with a probability of suceess or the value () with the probability of failure. This type of variable is called a Bernoulli or binary variable. The relationship between the predictor and the dependent variable is not a linear one, it is a logit transformation. An advantage of using logistic regression is that no assumptions about the distribution of the dependent variable need to be met.

One form of the logistic regression equation is:

$$
\operatorname{logit}\left(p_{i}\right)=\ln \left(\frac{p_{i}}{1-p_{i}}\right)=\beta_{0}+\beta_{1} x_{1, i}+\cdots+\beta_{k} x_{k, i}
$$

A particular element of $\mathrm{X}_{i}$ can be set to I for all $I$ to yield an intercept for the model. The unknown parameters $B_{f}$ are usually estimated by maximum likelihood. Interpretation of the $\mathrm{Bj}$ estimates is the additive effect on the log odds ratio, a unit change in the $j$ th explanatory variable. In the case of a dichotomous explanatory variable, gender, $e^{k}$ is the estimate or odds of having the outcome for examples males compared with females. The odds are a measure of effect size and are defined as the ratio of the probability of an event occurring in one group to the probably of it oceurring in another group. These groups might be men and women, or an experimental group and control group. If the 
probabilities of the event in each group are $p$ (first group) and $q$ (the second group), then the odds ratio is:

$$
\frac{p /(1-p)}{q /(1-q)}=\frac{p(1-q)}{q(1-p)}
$$

These results of the logistic regression are easy to interpret, as they are reported using odds ratios. An odds ratio of 1 indicates that the odds of exposure is the same in both groups. An odds ratio greater than one indicates that the condition or event is more likely to have occurred in the exposed group when compared to the non exposed group. In contrast an odds ratio less than one indicates that the condition or event is less likely to have oceurred in the exposed group.

In the model analyzing the association with chronic disease, the level of BMI was the independent variables or $\mathrm{x}$ while the existence of chronic disease was the dependent variable or y (e.g., the existence of hypertension yes/no). In the equations, age and gender were controlled for because of their well-evideneed relationship with health. Age was entered as al continuous variable and gender was included as a dummy variable. $A$ dummy variable allows for the addition of nominal or ordinal variables in a regression equation. The standard approach is to include the categorical variables in the regression equation by coding each level of each categorical variable as 0 or 1 . Conventionally 1 means the attribute of interest is present. A binary nominal variable such as gender may be coded 0 for male and 1 for female. 


\subsubsection{Poisson Regression Models}

Poisson regression models were developed to examine the predictive relationship between level of BMI and health services utilization. This regression technique was utilized as the dependent variables of interest; the number of physician and hospital visits, LOS and day surgeries per patient were measured as whole discrete numbers or counts. Health services utilization data does not generally follow a normal distribution and is often positively skewed to the right with many individuals having zero or little health services utilization and very few individuals having a large number of visits.

A Poisson distribution can be used to describe the discrete distribution of a number of occurrences or counts over time such as the number of visits to a physician in one year. There is no assumption that the dependent or independent variables are related linearly. There is no assumption of homogeneity of variance; that is variances are equal. The predictors or independent variables may be either continuous or categorical. A key characteristic of the Poisson distribution is that its mean is equal to its variance. ${ }^{213}$

The following section describes Poisson regression providing the relevant equations for clarification. If the response variable of interest has a Poisson distribution as in this case, the probability of observing $Y$ occurrences is calculated as: where $\mathrm{e}=2.71$ (approximately) and $\mu$ is the mean.

Probability $(Y)=e^{-u} u^{y}$, for any $Y=0,1,2 \ldots \ldots$ $\mathrm{Y}$ ! 
If $Y$ is a Poisson variable that has a mean of $\mu$ which depends on explanatory variables $\mathrm{X} 1$ and $\mathrm{X} 2$, then a Poisson $\log$-linear regression model states the natural $\log (\mathrm{ln})$ of the mean can be written as a linear function of the regression coefficients.

$$
\operatorname{Ln}(\mu)=\beta_{0}+\beta_{1} X_{1}+\beta_{2} X_{2}
$$

This can be extended to include any number of regression coefficients. For example if : $\mathrm{Y}=$ number of physician visits in a year; $\mathrm{X}_{1}=\mathrm{age}, \mathrm{X}_{2}=\mathrm{BMI}$ level

The interpretation of $B_{i}$ depends on whether $X_{1}$ is quantitative (age, heart rate) or categorical (level of $\mathrm{BMI})$. If $\mathrm{X}_{1}$ is quantitative the $\mathrm{B} 1$ tells us, assuming $\mathrm{X}_{2}$ remains fixed a 1 - unit increase in $X_{1}$ is associated with $\mathrm{K}$-fold increase in the mean of $y(i . e ., \mu$ ), where $\mathrm{K}=\mathrm{e}^{\beta}$. If $\mathrm{X}_{1}$ is age and $\mathrm{B}_{1}=0.069$, then increasing age by 1 year is estimated to be associated with $\mathrm{e}^{0.069}=1.07$-fold change or a $7 \%$ increase in the mean (average) number of physician visits $(Y)$. If $X_{1}$ is categorical the $B_{1}$ tells us that $X_{2}$ remains fixed, that $\mu$ (mean of $Y$ ) is $\mathrm{e}^{\beta}$ t times higher for the group represented by $\mathrm{X}_{1}$ than for baseline group. For example suppose $B_{1}=0.03$ then keeping $X_{2}$ fixed, the average number of physician visits $(Y)$ for a person with an obese level of $B M I$ is $\mathrm{e}_{1}^{\beta}=e^{0.03}=1.03$ times higher $(3 \%)$ than a person with a normal BMI.

There are two common problems with Poisson regression. First, in certain circumstances the observed variance can be greater than the mean and this is known as overdispersion. Dispersion is the spread of a set of observations. Overdispersion occurs in 
Poisson regression when the variance is greater than the mean. If the overdispersion is large, it may signal a problem with the model. If the overdispersion is moderate it can be corrected by using an adjusted standard error $=\mathrm{SE} *$ sqrt (D/df), where $D$ is scaled deviance, which for loglinear equals $G 2$ and $d f=$ the number of terms $n$ in the model not counting the constant. ${ }^{214}$

The second problem with Poisson regression is excess zeros. If there are two processes at work, one determining whether there are zero events or any events, and a Poisson process determining how many events there are, there will be more zeros than a Poisson regression would predict. An example would be the distribution of cigarettes smoked in an hour by members of a group where some individuals are non-smokers.

Both challenges were encountered in the study. There was moderate overdispersion that was corrected by the inclusion of a correction factor. The inclusion of a correction factor does not change the estimates of the equation, but does change the standard errors attached to the estimates as the correction formula demonstrates. In addition, as the HSU data had many zeros, the Poisson regressions were run with and without the zeros and no differences were found. In the Poisson regression models using Andersen's model as a guide, all the predisposing variables were included. Under enabling all the variables were included with the exception of 'regular doctor' as a high proportion of all BMI groups had access to a regular doctor. Under the category of Need, the number of chronic conditions and the health status variables were included; the inclusion in the model of the other 'need' variables did not impact the results. 


\subsubsection{Multiple Linear Regression Models}

Two multiple linear regression models were conducted to estimate the predictive relationship between level of BMI and the dependent variables of direct costs associated with physician and inpatient services considered to be continuous variables. As presented earlier, linear regression is a parametric statistic based on calculating averages and standard deviations using the parameters of a normal distribution. Linear regression uses the correlation between variables in order to find the best mathematical model for predicting one variable from another. As well, linear regression describes the straight line relationship between a predictor variable(s), plotted on the $x$-axis, and a response /outcome variable, plotted on the $y$-axis.

A simple linear regression equation is as follows:

$$
\mathrm{Y}=\mathrm{b}_{0}+\mathrm{b}_{1} \mathrm{x}_{1}+\mathrm{e} \text { where: }
$$

$b_{0}$ is the $Y$-intercept, and $b_{1}$ is the regression coefficient which defines the slope of the line for $\mathrm{x}_{1}$ the independent or explanatory variable. The dependent/response variable is ' $\mathrm{Y}$ ' and $e$ is a random variable called the error term. A multiple linear regression equation is similar to a simple linear regression equation other than that it includes more than one independent variable (i.e., $x_{2}, x_{3}$ etc.), for example:

$$
Y=b_{0}+b_{1} x_{1}+b_{2} x_{2}+b_{3} x_{3}+b_{4} x_{4}+e
$$

Multiple linear regression is a parametric statistic and is based on the parameters of the normal distribution. Therefore, several assumptions apply to the analysis. The sample size must be relatively large, and there should be between five and fifteen cases per independent variable. Independent variables should not be highly correlated or 
demonstrate multi collinearity. The residuals should have a linear relationship with the predicted scores. Only one response per dependent variable is allowed, therefore there should be no repeated measures. The variance for residuals about the predicted score should be the same for all seores.

Initial exploration via a histogram of the cost data, illustrated a distribution that was not quite normal as well as many values that were zero. However non-linearity of a continuous response variable can be corrected by a mathematical transformation in order that the data can be used in a linear model. In this scenario the dependent variable was $\log$ transformed to ensure the data met the criteria of normality. Modeling using multiple regression analysis was run on the natural $\log (\ln )$ of the response variable. One needs to be aware that any data transformation changes the meaning of the model parameters and their interpretation. Therefore caution should be made when interpreting the beta coefficients in the equation. A positive increase in a beta increases the natural log of the dependent variable.

Due to the number of zeros encountered in the dataset (i.e., those survey respondents who had not utilized either physician or hospital services), 0.1 was added to each individual cost so the natural log could be computed. The log of zero produces a value of "infinity" which cannot be used in any statistical analysis, therefore in order to use the information from these data points an arbitrary value of 0.1 was added to each zero value. This retains the data while keeping the value elose to zero. Although this produees a negative value, it allows the data to be used in the statistical analysis. In the case of physician costs, the analysis (running the natural log of the costs with the above 
procedure) reported approximately 200 individuals with negative costs. In terms of the hospital costs, running the natural log produced well over 1500 individuals with negative costs. A decision was made to include the individuals with the 200 negative cost values in the analysis of physician costs. ${ }^{218}$ As a check the multiple regression models were run with and without the 200 negative values and the results were similar. In terms of the hospital costs, a decision was made to include only those individuals who had an inpatient stay and an associated cost.

Standard multiple regression was used to determine if level of BMI was an independent predictor of direct healthcare costs. The chosen variables were entered into the model at the same time as it was not the focus of the current thesis to test Andersen's empirical model but rather to use the model to guide the choice of independent variables for the regression models. Variables controlled for in this regression model were similar to those variables controlled for by other researchers in this field when analyzing the relationship level of BMI and health services utilization. However, only those variables showing a significant relationship were included in the cost analysis as to determine more accurately the explained variance (i.e., the $\mathrm{R}^{2}$ of the model). These variables included; level of BMI, age, gender, education level, region of residence and smoking behavior. Due to the limited data available on the cost of surgical day care the cost analysis was not tested. This was due to the limited coding of the variable used to code for resource use of day surgeries - the DPG_W. The coding of surgical day care commenced in February 2002. Early descriptive analysis of this data demonstrated mainly zeros for this variable. 


\subsection{Chronic Disease as a Mediator Variable}

In the current thesis, regression equations were developed to examine the relationship between level of BMI and an individual's use of health services. However as other variables can affect the use of health services, these variables were taken into consideration both in the Poisson and multiple regression models. For the Poisson and multiple linear regression models it was hypothesized that the number of chronic conditions may act as a mediator variable. ${ }^{215}$ A mediator variable is a type of third variable situated between the independent and dependent variables. Mediating variables account for a percentage of the relationship between the predictive variable and the outcome variable. To determine if a variable may be classified as a mediator variable, statistical analysis is run between, (1) the predictor and the dependent variable (2) the mediator and the dependent variable, and (3) the predictor, mediating variable and the dependent variable. If a variable(s) is to be described as a mediator, the statistical analysis should demonstrate a decrease in the effect of the predictor variable, once the mediator variable is included. ${ }^{215}$ The relationship between the independent and dependent variables can be decomposed into two causal paths. For example in Figure 4.3, a BMI $\geq$ 30 may have a direct relationship with increased health services utilization, however this relationship may be mediated or attenuated with the addition of chronic disease into the model. In this relationship one of the paths links the independent variable to the dependent variable directly and the other path links the independent variable to the dependent variable indirectly. An indirect or mediated effect implies that the independent variable causes the mediator variable which in turn causes the dependent variable. If a 
variable is to be described as a mediator variable, the statistical analysis should demonstrate a decrease in the effect of the independent variable when the mediator is added to the model. ${ }^{215}$ 


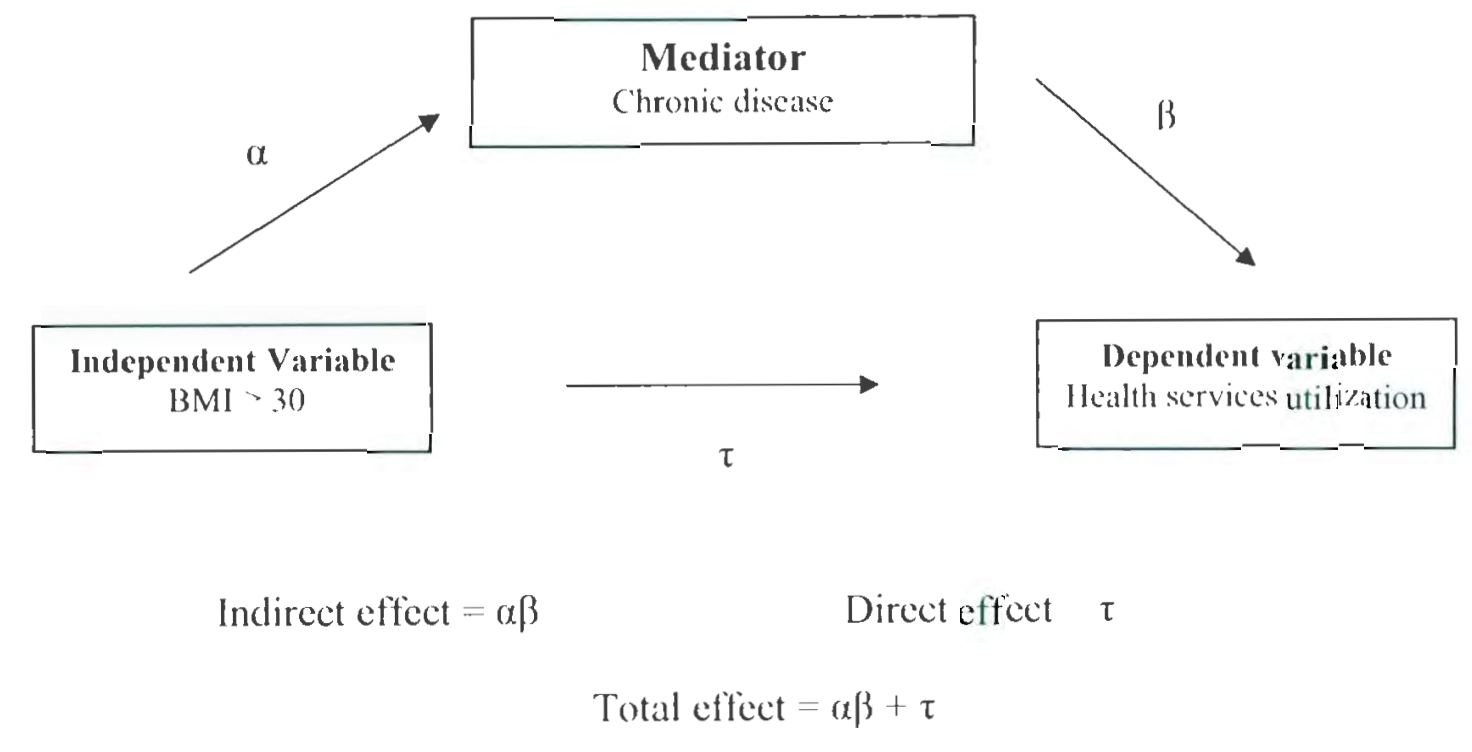

Figure 4.3 Illustration of a Mediator 
This is in contrast to a confounding variable, another type of third variable. A confounder is a type of third variable associated with both the independent variable and outcome variable. It is an extraneous factor that is in itself a determinant of the outcome of interest. ${ }^{213}$ Confounding occurs when the effects of two variables have not been separated and it is concluded that the effect observed is due to one variable rather than the combination of variables. A simple example would be the relationship between coffee drinking as an independent variable and its association with coronary heart disease as on outcome. Coffee drinking is associated with cigarette smoking and, cigarette smoking is in turn associated with heart disease. If one were to look only at the relationship between coffee drinking and heart disease, the strength of the relationship would be overestimated, as it may be the relationship with smoking that causes the increased incidence of heart disease (Figure 4.4). If known confounding variables are not controlled for in an analysis, the strength of the relationship under study may be overestimated and the resulting interpretation may be incorrect. 


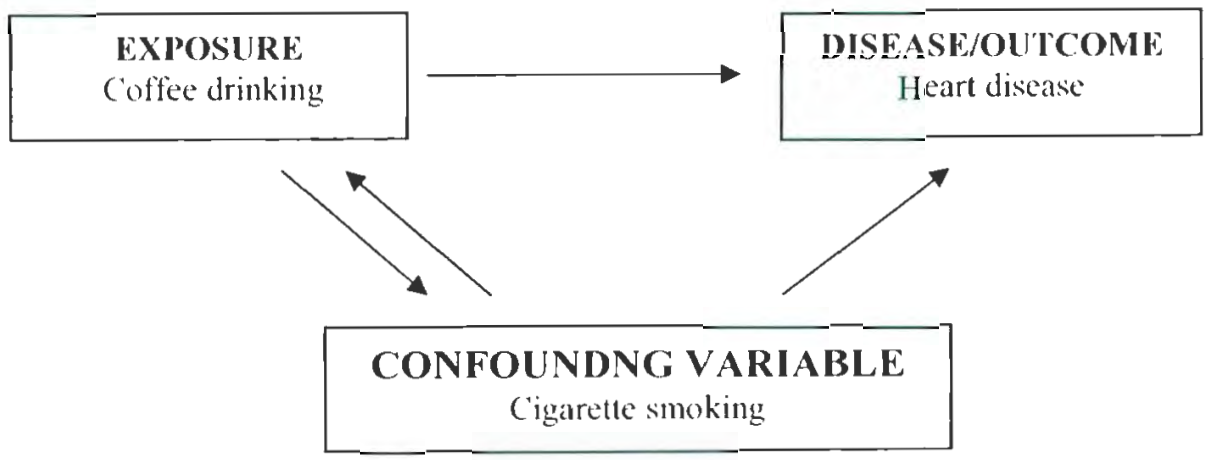

Figure 4.4 Illustration of a Confounder 
Confounders in the current study include those variables that have demonstrated a relationship with both the use of health services as well as BMI such as age, gender, income, education, smoking behavior and alcohol consumption. ${ }^{213,215}$ Confounding variables may distort the true relationship of the variables under study by being independently related to the outcome of interest. These variables are extraneous to the relationship under study and are often unequally distributed among the groups being compared such as age and gender. If these confounding variables are not controlled for in analysis, the strength of the relationship may be overestimated. Statistical analysis for observational studies can only control for known confounders, and there is always the possibility that an unknown or unmeasured confounder exists. In randomized control trials, where participants are randomly allocated to two groups (e.g., treatment or placebo), unknown confounders can be controlled.

Both types of variables are treated identically for statistical purposes and can only be distinguished on conceptual grounds. When a mediator or confounder is added to the analysis, each is quantified my measuring a change in the relationship between the independent variable and the dependent variable. It is hypothesized that the existence of chronic disease is a mediator variable within this relationship.

\subsection{Ethical Considerations}

This study was approved by the Human Investigation Committee at Memorial University of Newfoundland (Appendix F). Permission to use the Canadian Community Health Survey 1.1 "link file" was received from the Department of Health and Community Services, NL (Appendix G). Permission to access and use the 2000/2001 
CCHS 1.1 "share file", the Clinical Database Management System (CDMS) was received from the NL Centre for Health Information (Appendix H). Permission to use the fec-forservice physician claims database was received from the Medical Care Plan (Appendix I). All personal identifiers (name, address, telephone and health insurance number) were removed from the research database after linkages were conducted. An anonymous unique identifier number replaced the MCP number. The researcher was required to sign a standard oath of confidentiality. Access to the identities associated with the personal health information was limited to a co-investigator employed by NLCHI who also conducted the database linkages. All paper forms and printouts of electronic files were kept in a secure place and computer files related to the study were password protected and kept in a locked office. 


\section{Chapter 5 Results}

\subsection{Introduction}

This chapter presents the findings of the research study. Data analyses were performed using statistical software packages SPSS (Version 13.5) and 'R'. SPSS was used for the deseriptive analysis, logistic regression equations and multiple regression equations while ' $R$ ' was used to run the Poisson regression analysis (i.e., the count data models for prediction of health services utilization). The study findings are presented in the following four sections. Section 5.2 reports the secondary analysis of the 2000/20(0) CCHS (Tables 5.1 to 5.6). Section 5.3 reports the analyses of the measures of actual health serviees utilization (Tables $5.7,5.8$, Figure 5.1). Section 5.4 reports the results of

the analysis comparing self reported and actual visits to a physician (Table 5.9). Table 5.5 reports the prediction models deseribing the relationship between the "actual" level of health services utilization and the level of BMI (Tables 5.10-to 5.14). Section 5.6 describes the direct costs of obesity (Table 5.15, Figures $5.2,5.3$ ) and the multiple regression prediction estimates which described the relationship between level of BMI and the direct healtheare cost (Tables 5.16,5.17).

\subsection{The $2000 / 2001$ CCHS}

A total of 2366 survey respondents between 20 and 64 years of age met the initial criteria and were included in the study analysis: $47.2 \%$ were males and $52.8 \%$ were females with an average age of 41 years $(S D=11.67)$. The average $B M I$ was found to be $27.0 \mathrm{~kg} / \mathrm{m}^{2}$ with a range between $18.0 \quad 60.4 \mathrm{~kg} / \mathrm{m}^{2}$. The sample was divided into the appropriate BMI categories using the WHO classification system. A total of $548\left(23^{\circ} \%\right)$ 
survey respondents were classified as obese with BMI scores $\geq 30 \mathrm{~kg} / \mathrm{m}^{2}$. The obese group was further broken down into 407 obese (BMI $\geq 30 \mathrm{~kg} / \mathrm{m}^{2}$ and $<35 \mathrm{~kg} / \mathrm{m}^{2}$ ) and 141 morbidly obese (BMI $\geq 35 \mathrm{~kg} / \mathrm{m}^{2}$ ). A total of $916(39 \%)$ survey respondents were classified as overweight with BMI scores between 25.0 and $29.9 \mathrm{~kg} / \mathrm{m}^{2}$ and $881(37 \%)$ survey respondents were classified as normal weight with BMI scores between $18.5 \mathrm{~kg} / \mathrm{m}^{2}$ and $24.9 \mathrm{~kg} / \mathrm{m}^{2}$. Less than $1 \%(\mathrm{n}=21)$ of the study population was classified as underweight (BMI $<18.5 \mathrm{~kg} / \mathrm{m}^{2}$ ).

Table 5.1 summarizes the differences in selected characteristics stratified by BMI category. Due to the nature of the data (e.g., categorical and nominal), chi-squared tests were conducted to determine whether there were significant differences between the independent variables such as gender, age etc. and level of BMI. The p-values (the probability of obtaining the study result by chance) were reported according to the level of significance from high, to moderate, to low significance $\mathrm{p}<.001, \mathrm{p}<.01$ and $\mathrm{p}<.05$. Significant differences were found in the sample population between all categorical variables and BMI category with the exception of education. Morbidly obese individuals were more likely to be female, to be between 40 and 49 years of age, live in the area described as the Eastern Health Region, to be middle income earners and to be either married or in a common-law relationship. Obese individuals were similar to the morbidly obese group in relation to demographic variables with a few exceptions. The obese population were divided equally between men and women and were more likely to be slightly older than the morbidly obese group that is between 50 and 64 years of age. 
Table 5.1 CCHS Demographics by Level of BMI

\begin{tabular}{|c|c|c|c|c|c|c|c|c|c|}
\hline \multirow[t]{3}{*}{ Characteristic } & \multirow{2}{*}{\multicolumn{2}{|c|}{$\begin{array}{l}\text { Morbid } \\
\mathrm{n}=141 \\
\end{array}$}} & \multirow{2}{*}{\multicolumn{2}{|c|}{$\begin{array}{l}\text { Obese } \\
\mathbf{n}=\mathbf{4 0 7}\end{array}$}} & \multirow{2}{*}{\multicolumn{2}{|c|}{$\begin{array}{c}\text { Overweight } \\
\mathrm{n}=916\end{array}$}} & \multirow{2}{*}{\multicolumn{2}{|c|}{$\begin{array}{c}\text { Normal } \\
\mathrm{n}=\mathbf{8 8 1}\end{array}$}} & \multirow[t]{3}{*}{ p-value } \\
\hline & & & & & & & & & \\
\hline & $\mathbf{n}$ & $(\%)$ & $\mathbf{n}$ & $(\%)$ & $\mathbf{n}$ & $(\%)$ & $\mathbf{n}$ & $(\%)$ & \\
\hline \multicolumn{10}{|l|}{ Gender } \\
\hline male & 48 & (34) & 204 & (50) & 505 & (55) & 353 & (40) & $<.001$ \\
\hline Female & 93 & (66) & 203 & (50) & 411 & (45) & 528 & (60) & \\
\hline \multicolumn{10}{|l|}{ Age in years } \\
\hline 20-29 & 25 & (18) & 53 & (13) & 103 & (11) & 171 & (20) & $<.001$ \\
\hline $30-39$ & 33 & (23) & 100 & (25) & 234 & (26) & 237 & (27) & \\
\hline $40-49$ & 43 & (31) & 120 & (30) & 252 & (28) & 211 & (24) & \\
\hline $50-64$ & 40 & (28) & 134 & (33) & 327 & (36) & 262 & (30) & \\
\hline \multicolumn{10}{|l|}{ Health Region } \\
\hline Urban $(\geq 5000)$ & 96 & (68) & 241 & $(60)$ & 567 & (62) & 599 & (68) & $<.01$ \\
\hline Rural $(<5000)$ & 45 & (32) & 166 & (41) & 349 & (38) & 282 & (32) & \\
\hline Eastern $^{1}$ & 52 & (37) & 155 & (38) & 392 & (43) & 428 & (49) & $<.01$ \\
\hline Central & 32 & (23) & 78 & (19) & 158 & (17) & 126 & (14) & \\
\hline Western & 23 & (16) & 62 & (15) & 148 & (16) & 160 & (18) & \\
\hline Northern & 34 & (24) & 112 & (28) & 218 & (24) & 167 & (19) & \\
\hline \multicolumn{10}{|l|}{ Education } \\
\hline$<$ Secondary & 25 & (18) & 72 & (18) & 148 & (16) & 145 & (17) & NS \\
\hline Secondary ${ }^{2}$ & 33 & (24) & 87 & (22) & 174 & (19) & 168 & (19) & \\
\hline Trade school ${ }^{3}$ & 69 & (50) & 200 & (50) & 437 & (48) & 415 & (47) & \\
\hline University graduate & 12 & (9) & 43 & (11) & 149 & (16) & 148 & (17) & \\
\hline \multicolumn{10}{|l|}{ Income $^{4}$} \\
\hline Low & 7 & (5) & 28 & (7) & 42 & (5) & 75 & (9) & $<.001$ \\
\hline Middle & 120 & (88) & 301 & (77) & 666 & (75) & 617 & (75) & \\
\hline High & 9 & (7) & 64 & (16) & 175 & (20) & 128 & (16) & \\
\hline \multicolumn{10}{|l|}{ Marital Status } \\
\hline married/common-law & 94 & (67) & 304 & (75) & 708 & (77) & 562 & (63) & $<.001$ \\
\hline Unpartnered $^{5}$ & 47 & (33) & 103 & (25) & 208 & (23) & 317 & (36) & \\
\hline
\end{tabular}


Table 5.2 summarizes the differences in lifestyle and health status characteristics stratified by BMI. Lifestyle variables include: smoking, drinking behavior, level of physical activity and daily consumption of fruits and vegetables. Health Status variables may include subjective and objective measures of health. Self-perceived health, subjectively measures how an individual perceives his or her own health. Objective measures of health include questions on the number of disability days taken in the previous two weeks, and the presence of chronic conditions. In addition, the Health Utility Index (HUI), a derived variable that combines questions on activities of daily living objectively measures functional health. The HUI, a continuous variable is reported in the range 0 to 1 and is not normally distributed. The Kruskal-Wallis nonparametric test for $\mathrm{k}$-independent groups was conducted to determine if there were significant differences between the HUI and BMI category.

Morbidly obese individuals were more likely to have reported being inactive, occasional/never drinkers of alcohol and to consume $<5$ servings of fruits and vegetables per day. A high percentage of morbidly obese individuals reported being in excellent, very good or good health, although the percentage was significantly lower than the other BMI categories. The average HUI for the morbidly obese was high but again it was significantly lower than the other BMI groups. A larger percentage of morbidly obese individuals reported having been diagnosed with at least one chronic condition. 
Table 5.2 Lifestyle Characteristics and Health Status Variables by Level of BMI

\begin{tabular}{|c|c|c|c|c|c|c|c|c|c|}
\hline \multirow{3}{*}{ Characteristic } & \multirow{2}{*}{\multicolumn{2}{|c|}{$\begin{array}{l}\text { Morbid } \\
\mathrm{n}=141\end{array}$}} & \multirow{2}{*}{\multicolumn{2}{|c|}{$\begin{array}{l}\text { Obese } \\
n=407\end{array}$}} & \multirow{2}{*}{\multicolumn{2}{|c|}{$\begin{array}{c}\text { Overweight } \\
n=916\end{array}$}} & \multirow{2}{*}{\multicolumn{2}{|c|}{$\begin{array}{c}\text { Normal } \\
\mathrm{n}=\mathbf{8 8 1}\end{array}$}} & \multirow[t]{3}{*}{ p-value } \\
\hline & & & & & & & & & \\
\hline & n & $(\%)$ & n & $(\%)$ & $\mathbf{n}$ & $(\%)$ & $\mathbf{n}$ & $(\%)$ & \\
\hline \multicolumn{10}{|l|}{ Smoking } \\
\hline Daily/former & 80 & (57) & 240 & (59) & 556 & (61) & 506 & (57) & NS \\
\hline Occasional \pm & 28 & (20) & 68 & (17) & 148 & (16) & 162 & (18) & \\
\hline Never smoked & 33 & (23) & 99 & (24) & 212 & (23) & 213 & (38) & \\
\hline \multicolumn{10}{|l|}{$\begin{array}{l}\text { Physical activity } \\
\text { level }\end{array}$} \\
\hline Active/moderate & 38 & (27) & 141 & (36) & 355 & (40) & 366 & (43) & $<.01$ \\
\hline Inactive & 95 & (72) & 254 & (64) & 532 & (60) & 492 & (57) & \\
\hline \multicolumn{10}{|l|}{ Type of drinker } \\
\hline Regular/former & 85 & $(60)$ & 263 & (65) & 662 & (65) & 632 & (72) & $<.01$ \\
\hline Occasional/never & 56 & (40) & 144 & (35) & 254 & (35) & 249 & (28) & \\
\hline \multicolumn{10}{|l|}{$\begin{array}{l}\text { Consumption of } \\
\text { fruits/vegetables }\end{array}$} \\
\hline$<5$ servings/day & 115 & (83) & 300 & (74) & 684 & (75) & 597 & (68) & $<.001$ \\
\hline$\geq 5$ servings/day & 24 & (17) & 105 & (26) & 226 & (25) & 278 & (32) & \\
\hline Self-rated health & & & & & & & & & \\
\hline $\begin{array}{l}\text { excellent/very } \\
\text { good/good }\end{array}$ & 111 & (79) & 355 & (87) & 816 & (89) & 769 & (87) & $<.01$ \\
\hline fair/poor & 30 & (21) & 52 & (13) & 100 & (11) & 112 & (13) & \\
\hline $\begin{array}{l}\text { Health Utility Index } \\
\mathrm{n}, \text { mean (SD) }\end{array}$ & 139 & $.84(.23)$ & 404 & $.89(.19)$ & 908 & $.90(.17)$ & 873 & $.89(19)$ & $<.01$ \\
\hline $\begin{array}{l}\text { \# of disability days } \\
\mathrm{n} \text {, mean (SD) }\end{array}$ & 141 & $1.5(3.8)$ & 407 & $1.0(3.1)$ & 916 & $.77(2.6)$ & 881 & $.93(2.9)$ & NS \\
\hline $\begin{array}{l}\text { Presence of a } \\
\text { chronic condition } \\
\text { \# chronic } \\
\text { conditions }\end{array}$ & 107 & (76) & 260 & (64) & 545 & (60) & 530 & (60) & $<.01$ \\
\hline 0 & 34 & (24) & 147 & (36) & 371 & (41) & 349 & (40) & $<.001$ \\
\hline $1-3$ & 93 & (66) & 208 & (51) & 480 & (53) & 465 & (53) & \\
\hline$\geq 4$ & 14 & (10) & 52 & (13) & 64 & (7) & 67 & (7) & \\
\hline
\end{tabular}

Morbidly obese (BMI $\geq 35$ ), obese (BMI $>30)$, overweight $(25 \leq \mathrm{BMI}<30$ ), normal (BMI 18.5 $24.9), \pm$ includes occasional, always occasional and formerly occasional smokers 


\subsubsection{Chronic Conditions}

Table 5.3 summarizes the self reported prevalence of chronic conditions comparing morbidly obese, obese, overweight and normal weight groups. Morbidly obese and obese groups were significantly more likely to have reported being diagnosed with cerebrovascular and cardiovascular discases, hypertension, endocrine discases including diabetes and a gastrointestinal or neurological disease. The self reported prevalence of cercbrovascular and cardiovascular diseases was $27.7 \%$ in the morbidly obese, $26.3 \%$ in the obese, $17.8 \%$ in the overweight and $9.2 \%$ in the normal weight The self reported prevalence of hypertension increased from $6.7 \%$ in the normal weight to $26.2 \%$ in the morbidly obese group. The self reported prevalenee of endocrine discases was $7.5 \%$ in the normal weight group, $9.1 \%$ in the overweight group, $12.3 \%$ in the obese group and $17.0 \%$ in the morbidly obese group. As well an increase in the prevalence of self reported cliabetes was reported from $2.7 \%$ in the nomal weight group to $11.3 \%$ in the morbidly obese group. There were significant differenees in the self reported prevalence of the gastrointestinal and neurological discases across levels of BMI. There were no differences seen across the levels of BMI with the prevalenee of disease for rheumatology, pulmonary, asthma or allergies. 
Table 5.3 Self-Reported Prevalence of Chronic Conditions by Level of BMI

\begin{tabular}{|c|c|c|c|c|c|c|c|c|c|}
\hline \multirow{3}{*}{$\begin{array}{c}\text { Chronic } \\
\text { Condition }^{1}\end{array}$} & \multirow{2}{*}{\multicolumn{2}{|c|}{$\begin{array}{l}\text { morbid } \\
n=141\end{array}$}} & \multirow{2}{*}{\multicolumn{2}{|c|}{$\begin{array}{c}\text { obese } \\
\mathrm{n}=407\end{array}$}} & \multirow{2}{*}{\multicolumn{2}{|c|}{$\begin{array}{c}\text { overweight } \\
\mathrm{n}=916\end{array}$}} & \multicolumn{2}{|c|}{ normal } & \multirow{3}{*}{$\begin{array}{c}\mathrm{p}- \\
\text { value }\end{array}$} \\
\hline & & & & & & & \multicolumn{2}{|c|}{$\mathrm{n}=881$} & \\
\hline & $\mathrm{n}$ & $(\%)$ & $\underline{n}$ & $(\%)$ & $\mathrm{n}$ & $(\%)$ & $\mathrm{n}$ & $(\%)$ & \\
\hline $\begin{array}{l}\text { Cerebrovascular and } \\
\text { CVD }^{2}\end{array}$ & 39 & $(27.7)$ & 107 & $(26.3)$ & 163 & $(17.8)$ & 82 & $(9.2)$ & $<.001$ \\
\hline $\begin{array}{l}\text { High blood } \\
\text { pressure }\end{array}$ & 37 & $(26.2)$ & 97 & $(23.8)$ & 133 & $(14.5)$ & 60 & $(6.7)$ & $<.001$ \\
\hline Endocrine diseases ${ }^{3}$ & 24 & $(17.0)$ & 50 & (12.3) & 83 & $(9.1)$ & 66 & $(7.5)$ & $<.01$ \\
\hline $\begin{array}{l}\text { Diabetes } \\
\text { mellitus }\end{array}$ & 16 & (11.3) & 32 & $(7.8)$ & 41 & $(4.5)$ & 24 & $(2.7)$ & $<.001$ \\
\hline $\begin{array}{l}\text { Gastrointestinal } \\
\text { diseases }^{4}\end{array}$ & 9 & $(6.4)$ & 23 & $(5.6)$ & 24 & $(2.6)$ & 39 & $(4.4)$ & $<.05$ \\
\hline $\begin{array}{l}\text { Rheumatologic } \\
\text { diseases }^{5}\end{array}$ & 42 & $(29.8)$ & 134 & (32.9) & 259 & $(28.3)$ & 251 & $(28.5)$ & NS \\
\hline Pulmonary diseases ${ }^{6}$ & 15 & $(10.6)$ & 33 & $(8.1)$ & 56 & $(6.1)$ & 70 & (7.9) & NS \\
\hline Asthma & 12 & $(8.5)$ & 24 & $(5.9)$ & 44 & $(4.8)$ & 54 & $(6.2)$ & NS \\
\hline $\begin{array}{l}\text { Neurological } \\
\text { diseases }^{\gamma}\end{array}$ & 24 & $(17.0)$ & 46 & (11.3) & 79 & $(8.6)$ & 117 & (13.3) & $<.01$ \\
\hline Allergies $^{8}$ & 33 & $(23.4)$ & 79 & (19.4) & & & 201 & $(22.8)$ & NS \\
\hline
\end{tabular}

\footnotetext{
'In the Canadian Community Health Survey 1.1, survey respondents were asked if they had ever been diagnosed by a doctor with a list of 25 chronic conditions. This list was collapsed into a smaller list of chronic conditions based on the disease (as per a medical doctor).

${ }^{2}$ includes high blood pressure, heart disease (including heart attack, angina and congestive heart failure) and effects of stroke

${ }^{3}$ includes diabetes mellitus and thyroid condition, ${ }^{4}$ includes bowel disorder (Crohn's or colitis) and stomach or intestinal ulcers ${ }^{5}$ includes fibromyalgia, arthritis or rheumatism and back problems, ${ }^{6}$ includes chronic bronchitis, emphysema or chronic obstructive pulmonary disease, ${ }^{7}$ includes migraine headaches, epilepsy, Alzheimer's disease or dementia, Parkinson's disease, multiple sclerosis and chronic fatigue syndrome ${ }^{8}$ including food, other allergies and multiple chemical sensitivities

NS, not significant for $\mathrm{p}<.05$
} 


\subsubsection{Self Reported Health Services Utilization}

Respondents were asked several questions pertaining to their health care utilization patterns in the $2000 / 2001$ CCHS. Table 5.4 summarizes and compares the respondents' healthcare utilization patterns. Interviewers asked questions requiring either a 'yes or no' response (e.g., have you received physician services in the pervious 12 months or do you have a regular family doctor?). As well, questions were asked about the level of healthcare utilization (e.g., the number of visits to a health professional or the number of nights spent in a hospital, institution or convalescence home). There were significant differences identified in the number of visits to a GP in the previous 12 months. The morbidly obese and the obese group reported having seen a GP a median of four and three times, respectively, compared to the normal and overweight groups who reported a median of two visits for a GP. However, there were no significant differences seen in any other utilization of healthcare services across the weight categories.

More than $75 \%$ of individuals in all levels of BMI reported having a regular doctor and having received physician services in the previous 12 months. Approximately $50 \%$ of the normal, overweight, obese groups and $40 \%$ of the morbidly obese group reported having received hospital services in the previous 12 months. Approximately $10 \%$ of the morbidly obese, obese and normal weight groups and $7 \%$ of the overweight group reported having been in hospital overnight. As well, $10 \%$ of the morbidly obese group reported having consulted a mental health professional. 
Table 5.4 Subjective Healthcare Resource Use Indicators in Previous 12 months by Level of BMI

\begin{tabular}{|c|c|c|c|c|c|c|c|c|c|}
\hline \multirow[t]{2}{*}{$\begin{array}{l}\text { Measure of } \\
\text { Utilization }\end{array}$} & \multicolumn{2}{|c|}{$\begin{array}{l}\text { Morbid } \\
n=141\end{array}$} & \multicolumn{2}{|c|}{$\begin{array}{l}\text { Obese } \\
\mathbf{n}=\mathbf{4 0 7}\end{array}$} & \multirow{2}{*}{\multicolumn{2}{|c|}{$\begin{array}{c}\text { Overweight } \\
n=916\end{array}$}} & \multicolumn{2}{|c|}{$\begin{array}{c}\begin{array}{c}\text { Normal } \\
n=881\end{array}\end{array}$} & \multirow[t]{2}{*}{$\begin{array}{c}\text { p- } \\
\text { value }\end{array}$} \\
\hline & $\mathrm{n}$ & $\%$ & $\mathrm{n}$ & $\%$ & & & $\mathrm{n}$ & $\%$ & \\
\hline $\begin{array}{l}\text { Have a regular } \\
\text { doctor }^{1}\end{array}$ & 113 & 80.1 & 317 & 77.9 & 726 & 79.3 & 710 & 80.6 & NS \\
\hline 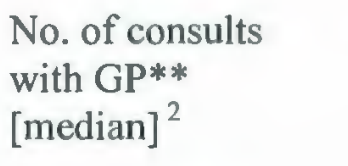 & 4.0 & {$[2.0,6.0]$} & 3.0 & {$[1.0,5.0]$} & 2.0 & {$[1.0,4.0]$} & 2.0 & {$[1.0,4.0]$} & 0.001 \\
\hline $\begin{array}{l}\text { No. of consults } \\
\text { with other medical } \\
\text { doctor [median] }{ }^{2}\end{array}$ & 0.0 & {$[0,0]$} & 0.0 & {$[0,0]$} & 0.0 & {$[0.0]$} & 0.0 & {$[0,1.0]$} & NS \\
\hline $\begin{array}{l}\text { Consulted mental } \\
\text { health provider' }\end{array}$ & 15 & 10.7 & 27 & 6.7 & 50 & 5.5 & 70 & 8.0 & NS \\
\hline $\begin{array}{l}\text { Received hospital } \\
\text { services } 1,3\end{array}$ & 12 & 41.4 & 51 & 52.6 & 93 & 49.7 & 97 & 50.5 & NS \\
\hline $\begin{array}{l}\text { Hospitalized } \\
\text { overnight }^{\prime}\end{array}$ & 14 & 9.9 & 39 & 9.6 & 65 & 7.1 & 81 & 9.2 & NS \\
\hline $\begin{array}{l}\text { No. of nights spent } \\
\text { in hospital } \\
\text { [median] }\end{array}$ & 4 & {$[2.0,14.0]$} & 4.0 & {$[1.0,7.0]$} & 4.0 & {$[2.0,7.0]$} & 4.0 & {$[2.0,7.5]$} & NS \\
\hline $\begin{array}{l}\text { Received } \\
\text { physician services }\end{array}$ & 27 & 93.1 & 79 & 81.4 & 156 & 83.4 & 161 & 83.9 & NS \\
\hline
\end{tabular}




\subsubsection{Logistic Estimates: Associations with Obesity}

This section explores the predictive relationship between demographic, health status and lifestyle variables and categories of obesity, as well as the predictive relationship between level of $\mathrm{BMI}$ and self reported chronic disease in the NL population (Tables 5.5, 5.6). These relationships were examined using logistic regression models.

Table 5.5 examines the demographic/ lifestyle and health status associations with being morbidly obese and obese in NL. Two logistic regression models were run for each set of independent variables. The outcome variable was dichotomous for each model $(1=$ morbid obesity; $0=$ normal weight) and ( $1=$ obese; $0=$ normal weight). Each logistic model was adjusted for age and gender by including in each equation, age as a continuous variable and gender as a dummy variable (male $=1$, female $=0$ ). Non significant findings were demonstrated by the inclusion of one in the $95 \%$ confidence interval and are reported in Table 5.5. The morbidly obese group was more likely to live in the Central and Northern health regions, to be inactive, to consume $<5$ servings of fruits/vegetables, to report poor or fair health, difficulty with activities and to be diagnosed with $\geq 4$ chronic conditions compared with the normal weight group. The morbidly obese group was less likely to be in a high income bracket, smoke and drink regularly. The obese group were more likely to live outside St. John's, have secondary or < secondary education, to be married, to be inactive, consume $<5$ servings of fruits/vegetables per day, to report being in poor or fair health, to have difficulty with activities and to have been diagnosed with $\geq 4$ chronic conditions. The obese group was less likely to drink or smoke regularly compared to the normal weight group. 
Table 5.5 Logistic Regression: Associations with Morbid obesity and Obesity adjusting for age and gender

\begin{tabular}{|c|c|c|c|c|}
\hline \multirow[t]{2}{*}{ Variable } & \multicolumn{2}{|c|}{ Morbid Obesity } & \multicolumn{2}{|c|}{ Obese } \\
\hline & Adjusted OR & $95 \% \mathrm{CI}$ & Adjusted OR & $95 \% \mathrm{CI}$ \\
\hline \multicolumn{5}{|l|}{ Health Region ${ }^{1}$} \\
\hline Eastern & 1.11 & $.621-1.99$ & 1.54 & $1.10-2.16$ \\
\hline Central & 2.15 & $1.25-3.73$ & 1.54 & $1.10-2.16$ \\
\hline Western & 1.24 & $.689-2.22$ & 2.21 & $1.56-3.14$ \\
\hline Northern & 1.74 & $1.02-2.96$ & 2.27 & $1.64-3.15$ \\
\hline \multicolumn{5}{|l|}{ Education $^{2}$} \\
\hline Sec, $<$ Secondary & 1.27 & $.87-1.88$ & 1.26 & $1.24-1.29$ \\
\hline \multicolumn{5}{|l|}{ Income $^{3}$} \\
\hline Low & .70 & $.42-1.17$ & .69 & $.45-1.05$ \\
\hline High & .55 & $.36-.85$ & .82 & $.60-1.12$ \\
\hline \multicolumn{5}{|l|}{ Marital status $^{4}$} \\
\hline $\begin{array}{l}\text { Married/common- } \\
\text { law }\end{array}$ & 1.11 & $.76-1.62$ & 1.66 & $1.62-1.69$ \\
\hline \multicolumn{5}{|l|}{ Smoking ${ }^{5}$} \\
\hline Daily & .51 & $.33-.78$ & .64 & $.63-.67$ \\
\hline \multicolumn{5}{|l|}{ Physical activity level ${ }^{6}$} \\
\hline Inactive & 1.82 & $1.22-2.73$ & 1.56 & $1.53-1.59$ \\
\hline \multicolumn{5}{|l|}{ Type of drinker ${ }^{7}$} \\
\hline Regular & .49 & $.34-.71$ & .59 & $.57-.60$ \\
\hline \multicolumn{5}{|l|}{$\begin{array}{l}\text { Consumption of } \\
\text { fruits/vegetables }\end{array}$} \\
\hline$<5$ servings/day & 2.39 & $1.50-3.81$ & 1.58 & $1.55-1.62$ \\
\hline $\begin{array}{l}\text { Self-perceived health } \\
\text { poor/fair }\end{array}$ & 1.99 & $1.20-3.01$ & 1.33 & $1.29-1.37$ \\
\hline $\begin{array}{l}\text { Difficulty with } \\
\text { activities (yes) }\end{array}$ & 2.11 & $1.22-3.66$ & 1.90 & $1.86-1.95$ \\
\hline$\geq 4$ chronic conditions & 1.67 & $1.63-1.71$ & 1.67 & $1.63-1.71$ \\
\hline
\end{tabular}




\subsubsection{Obesity as a Predictor of Chronic Disease}

This section examines the level of BMI as a predictor of self reported chronic disease in the NL population. Logistic regression models, adjusting for age and sex were developed. The independent variables in the model were level of BMI, age and sex. The dependent or outcome variable was dichotomous: the presence or absence of chronic discase where yes $=1$ and no $=0$ (Table 5.6) .

The likelihood of having reported being diagnosed with the following chronic conditions; cercbro/cardiovascular, hypertension, endocrine conditions and diabetes increased as the level of BMI increased. The strength of this relationship was demonstrated by the increasing odds ratios. $\Lambda$ s the corresponding confidence intervals did not include one, these findings were significant. For example, the overweight, obese and morbidly obese groups reported odds ratios (OR) of $2.91[95 \% \mathrm{Cl}(2.82,3.0)]$, OR 5.39 $[95 \% \mathrm{Cl}(5.2,5.58)]$ and an OR $8.24[95 \% \mathrm{CI}(7.86,8.63)]$ times more likely to have reported been diagnosed with hypertension compared to the normal weight group. Analysis of the remaining chronic discases (c.g., gastrointestinal, rheumatologic, pulmonary, asthma, neurological and allergies) did not demonstrate a clear dose-response relationship with increasing levels of BMI. 
Table 5.6 Logistic Regression: Level of BMI as a Predictor of Self-Reported Chronic Disease, adjusting for age and sex

\begin{tabular}{|c|c|c|c|}
\hline Chronic comorbidity $^{1}$ & Level of $\mathrm{BMI}^{2}$ & Odds Ratio & $95 \% \mathrm{CI}$ \\
\hline $\begin{array}{l}\text { Cerebro/cardiovascular } \\
\text { diseases }^{2}\end{array}$ & $\begin{array}{l}\text { overweight } \\
\text { obese } \\
\text { morbid }\end{array}$ & $\begin{array}{l}2.63 \\
4.24 \\
6.10\end{array}$ & $\begin{array}{l}2.56-2.71 \\
4.10-4.38 \\
5.84-6.38\end{array}$ \\
\hline High blood pressure & $\begin{array}{l}\text { overweight } \\
\text { obese } \\
\text { morbid }\end{array}$ & $\begin{array}{l}2.91 \\
5.39 \\
8.24\end{array}$ & $\begin{array}{l}2.82-3.00 \\
5.20-5.58 \\
7.86-8.63\end{array}$ \\
\hline Endocrine diseases ${ }^{3}$ & $\begin{array}{l}\text { overweight } \\
\text { obese } \\
\text { morbid }\end{array}$ & $\begin{array}{l}1.11 \\
1.44 \\
2.45\end{array}$ & $\begin{array}{l}1.07-1.14 \\
1.39-1.50 \\
2.39-2.51\end{array}$ \\
\hline Diabetes mellitus & $\begin{array}{l}\text { overweight } \\
\text { obese } \\
\text { morbid }\end{array}$ & $\begin{array}{l}1.44 \\
2.44 \\
5.41\end{array}$ & $\begin{array}{l}1.37-1.50 \\
2.32-2.56 \\
5.09-5.76\end{array}$ \\
\hline $\begin{array}{l}\text { Gastrointestinal } \\
\text { diseases }^{4}\end{array}$ & $\begin{array}{l}\text { overweight } \\
\text { obese } \\
\text { morbid }\end{array}$ & $\begin{array}{l}.549 \\
1.25 \\
1.47\end{array}$ & $\begin{array}{l}.326-.926 \\
.732-2.12 \\
.696-3.12\end{array}$ \\
\hline $\begin{array}{l}\text { Rheumatologic } \\
\text { diseases }^{5}\end{array}$ & $\begin{array}{l}\text { overweight } \\
\text { obese } \\
\text { morbid }\end{array}$ & $\begin{array}{l}.948 \\
1.27 \\
.969\end{array}$ & $\begin{array}{l}.930-.966 \\
1.24-1.30 \\
.934-1.01\end{array}$ \\
\hline Pulmonary diseases ${ }^{6}$ & $\begin{array}{l}\text { overweight } \\
\text { obese } \\
\text { morbid }\end{array}$ & $\begin{array}{l}.924 \\
1.23 \\
1.72\end{array}$ & $\begin{array}{l}.894-.955 \\
1.18-1.28 \\
1.64-1.81\end{array}$ \\
\hline Asthma & $\begin{array}{l}\text { overweight } \\
\text { obese } \\
\text { morbid }\end{array}$ & $\begin{array}{l}.879 \\
1.05 \\
1.36\end{array}$ & $\begin{array}{l}.580-1.33 \\
.635-1.17 \\
.708-2.63\end{array}$ \\
\hline Neurological diseases $^{7}$ & $\begin{array}{l}\text { overweight } \\
\text { obese } \\
\text { morbid }\end{array}$ & $\begin{array}{l}.710 \\
.916 \\
1.28\end{array}$ & $\begin{array}{l}.521-.966 \\
.633-1.33 \\
.784-2.08\end{array}$ \\
\hline Allergies $^{8}$ & $\begin{array}{l}\text { overweight } \\
\text { obese } \\
\text { morbid }\end{array}$ & $\begin{array}{l}.991 \\
.937 \\
1.21\end{array}$ & $\begin{array}{l}.971-1.01 \\
.912-.962 \\
1.08-1.16\end{array}$ \\
\hline
\end{tabular}

\footnotetext{
In the Canadian Community Health Survey 1.1, respondents were asked if they had been diagnosed by a doctor with a list of 25 chronic conditions. This list has been collapsed into chronic conditions based on the disease (as per a medical expert RA), ${ }^{2}$ has high blood pressure, heart disease (including heart attack, angina and congestive heart failure) and effects of stroke ${ }^{3}$ includes thyroid condition, ${ }^{4}$ includes bowel disorder (Crohn's or colitis) and stomach or intestinal ulcers ${ }^{5}$ includes Fibromyalgia, arthritis or rheumatism and back problems, ${ }^{6}$ includes chronic bronchitis, emphysema or chronic obstructive pulmonary disease, ${ }^{7}$ includes migraine headaches, epilepsy, Alzheimer's disease or dementia, Parkinson's disease, Multiple sclerosis and chronic fatigue syndrome

${ }^{8}$ including food, other allergies and multiple chemical sensitivities ${ }^{2}$ referent category normal weight
} 


\subsection{Measures of Actual Health Services Utilization}

The linkage of the health survey to the MCP and the CDMS databases were successful for 2177 survey respondents who provided valid health insurance numbers. These individual health insurance numbers were linked to the MCP and CDMS databases to obtain physician and hospital utilization.

\subsubsection{Medical Care Plan}

The descriptive results of this analysis are presented in Table 5.7. This table is categorized into physician consults, consults with a GP and consults with a specialist. A total of $94 \%$ of the survey respondents (2036) with a valid MCP had at least one visit with either a GP or specialist doctor, including a radiology assessment over the five-year study period 1998-2003. Of the total survey respondents with a valid MCP number, $84 \%$ (1838) had at least one visit with a GP and $85 \%$ (1858) had at least one visit with a specialist doctor. A significant difference was identified in terms of total utilization of physician services across the BMI categories. The morbidly obese group reported a much higher median number of visits (median 31) compared to the other BMI categories. When physician consults were separated into GP or specialist visits, a significant relationship remained only with the morbidly obese group for consults with a GP. A significant difference was seen between the morbidly obese group and all other BMI groups in terms of GP consults. The morbidly obese group reported a median of 22 visits to a GP compared to obese (median 17), overweight (median 16) and normal weight (median 17). Although, there were no significant differences across $\mathrm{BMl}$ categories and visits to a specialist doctor, the morbidly obese group did report a higher median than the 
other three BMI categories (median 9). In all cases, the relationship among BMI categories appears to be non-linear (i.e., the number of physician consults does not increase linearly with increasing BMI category). The relationship appears to be jor ushaped. The normal weight group reported a higher median number of visits to a physician compared to the overweight group. The overweight group reported a fewer median number of visits compared to the obese group. The obese group also reported a fewer number of median visits compared to the morbidly obese group (Figure 5.1 ). 
Table 5.7 Actual Physician Health Services Utilization by Level of BMI

\begin{tabular}{|c|c|c|c|c|}
\hline BMI category' & $\begin{array}{c}\text { Morbidiy } \\
\text { Obese }\end{array}$ & Obese & Overweight & Normal \\
\hline $\begin{array}{l}\text { Total physician consults } \\
(n=2036)\end{array}$ & 131 & 388 & 841 & 817 \\
\hline $\mathrm{n}(\%)$ & $127(97)$ & $360(93)$ & $783(93)$ & $766(94)$ \\
\hline median $\left[25^{\text {th }}, 75^{\text {th }} \%\right]^{*}$ & $31[14,52]$ & $24[10,45]$ & $21[8,45]$ & $24[10,48$ \\
\hline \multicolumn{5}{|l|}{ GP consults } \\
\hline$n(\%)$ & $114(87)$ & $312(80)$ & $695(83)$ & $717(88)$ \\
\hline median $\left[25^{\text {th }}, 75^{\text {th }} \%\right]^{*}$ & $22[10,37]$ & $17[7,29]$ & $16[6,28]$ & $17[7,28]$ \\
\hline $\begin{array}{l}\text { Specialist consults } \\
\text { n }(\%)\end{array}$ & $121(92)$ & $331(85)$ & $71.3(85)$ & $693(85)$ \\
\hline median $\left[25^{\text {th }}, 75^{\text {th }} \%\right]$ & $9[5,21]$ & $7[4,22]$ & $6[4,19]$ & $7[4.20]$ \\
\hline
\end{tabular}

Sample size varies based on number of valid MCP numbers in each BMI category 'BMI categories: normal (18.5-24.9); overweight (25-29.9); obese (30)-34.9); morbidly obese $(\geq 35.0),{ }^{*} p<.05$ 
Physician Utilization (GP and specialist) 1998-2003

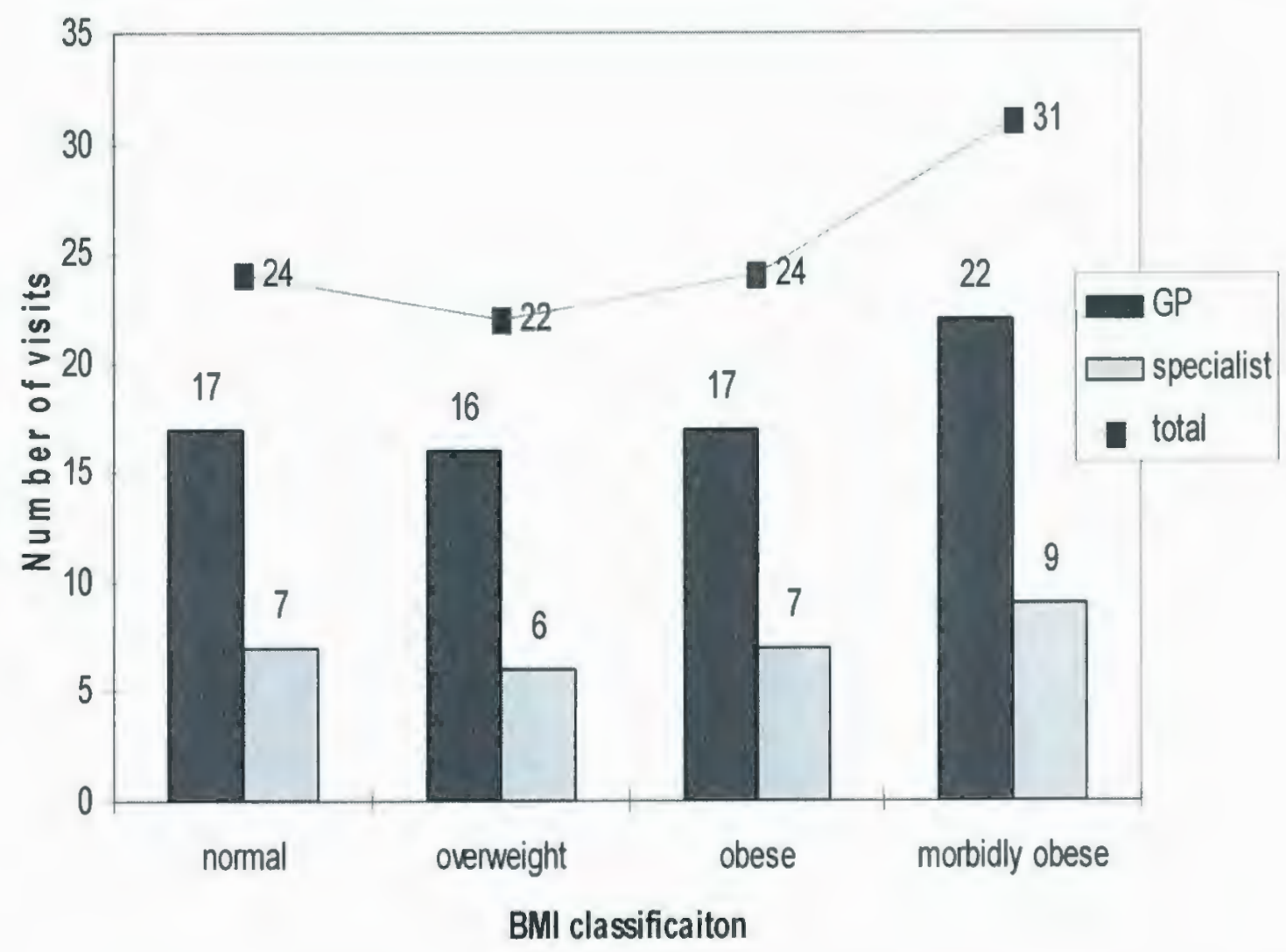

Figure 5.1 Physician Utilization (GP and specialist) by Level of BMI 


\subsubsection{Descriptive Statistics: Hospital Separations}

Table 5.8 presents the descriptive statistics for hospital separations (e.g., inpatient visits, length of stay and surgical day care cases). A total of 547 (25\%) respondents with a valid MCP number had at least one overnight stay in hospital and a total of $551(25 \%)$ had at least one surgical day care procedure over the five-year study period. For acute hospitalizations, there were no differences between levels of BMI for length of stay per episode, median number of visits per patient or average RIW (resoureeintensity-weight for inpatient stay). For surgical day care there were no differences between levels of BMI for either the median number of day surgeries per patient or the average DPG_W (i.e. the day procedure group resource-intensity-weight) per patient. 
Table 5.8 Hospital Health Services Utilization by Level of BMI

\begin{tabular}{|c|c|c|c|c|}
\hline BMI category ${ }^{1}$ & $\begin{array}{l}\text { Morbidly } \\
\text { Obese }\end{array}$ & Obese & Overweight & Normal \\
\hline \multicolumn{5}{|l|}{ Acute hospitalizations } \\
\hline $\begin{array}{l}\text { mean }(\mathrm{SD}) \\
\text { episode/patient }\end{array}$ & $2(2)$ & $2(1)$ & $2(1)$ & $2(2)$ \\
\hline $\begin{array}{l}\text { median }\left[25^{\text {th }}, 75^{\text {th }} \%\right] \\
\text { episode/patient }\end{array}$ & $1.0[1,2]$ & $1.0[1,2]$ & $1.0[1,2]$ & I.0 $.0[1,2]$ \\
\hline $\begin{array}{l}\text { median } \operatorname{LOS}^{2} \text { per episode } \\
{\left[25^{\text {th }}, 75^{\text {th }} \%\right]}\end{array}$ & $4[1,10]$ & $3[1,7]$ & $3[1,7]$ & $3[1,7]$ \\
\hline mean $\mathrm{RIW}^{3} \pm \mathrm{SD}$ & $1.60(1.62)$ & $1.43(1.61)$ & $1.93(3.26)$ & $2.44(5.08)$ \\
\hline \multicolumn{4}{|l|}{ Surgical day care cases } & 201 \\
\hline median $\left[25^{\text {th }}, 75^{\text {th }} \%\right]$ & $1[1,2]$ & $1[1,2]$ & $1[1,2]$ & $1[1,2]$ \\
\hline mean DPG_w ${ }^{4} \pm S D$ & $.26(.30)$ & $.78(1.52)$ & $.61(1.29)$ & $.55(.930)$ \\
\hline \multicolumn{5}{|c|}{$\begin{array}{l}\text { Sample size varies based on number of valid MCP numbers in each BMI category } \\
\text { I BMI categories: normal (18.5-24.9); overweight }(25-29.9) \text {; obese }(30-34.9) \text {; morbidly } \\
\text { obese }(\geq 35.0) ;{ }^{2} \text { LOS, length of stay; }{ }^{3} \text { RIW, resource intensity weight; }{ }^{4} \text { surgical day } \\
\text { care cases (weight DPG_W - available from February } 2002)\end{array}$} \\
\hline
\end{tabular}




\subsection{Comparison of Self Reported and Actual Visits to a Physician}

Using student t-tests, the means were compared for self reported visits made to a 'regular family doctor' and to 'another doctor' in the previous 12 months to the actual FFS physician visits made to both a GP and specialist over a 12 month period. (A comparison of medians provided similar results). As the actual FFS data included five years of data, for comparison purposes the total sum of FFS visits was divided by five for an annual average and these numbers were compared to the self reported data covering a 12 month period. A t-test comparing the means of self reported and actual GP visits was conducted separately on each level of BMI (morbidly obese, obese, overweight, normal weight) and within different geographical health regions. The results are presented for: 1) the province as a whole, 2) the urban and rural geographical regions and 3) the four regional health boards. These results are presented in Table 5.9 and Appendix E: Tables 3-4.

At a provincial level, there were no significant differences found comparing self reported and actual visits to a GP across levels of BMI (Table 5.9). In addition similar results were found when comparing self reported and actual GP visits between the rural and urban regions as well as within the specific health boards with the exception of the Northern Health Board. A significant difference was found between the number of actual FFS GP visits and those self reported by the morbidly obese in the Northern Health Board. (Appendix E: Table 4d). Due to limited sample size the regression analysis conducted was at a provincial level. 
In contrast to the GP visits there were significant differences found between self reported and actual FFS visits when comparing visits made to a specialist. An evaluation of the types of data collected through both sources: self reported and actual demonstrated that the data were not directly comparable. For example the FFS actual data provides information on various types of specialist services (e.g., office/home visits, in-patient consultation, out-patient and emergency consultation, diagnostic and therapeutic procedures, in-hospital diagnostic procedures, radiology and surgical procedures) and was not limited to visits per se. Office and home visits contributed only $15 \%$ of specialist visits. (Appendix E: Table 2). In comparison the type of specialist visit collected in the survey, the question is aimed at collecting information on number of visits made to 'another doctor' (other than the respondent's family doctor). This survey question would be unlikely to pick up information on surgical, diagnostic or therapeutic procedures. The linked database allows a greater capture of services provided by specialists although it must be noted that the data on salaried specialists is missing. In general the average number of office visits to a specialist was quite low across all BMI groups and within all geographies and the descriptive statistics demonstrated that there were no significant differences across levels of BMI (Tables 5.4, 5.7). This pattern observed was similar whether using the self reported data or the linked dataset. Therefore for analysis purposes it was decided to stick with the larger dataset of actual data as it provided five years of data versus only one year of data collected in the survey. At this point there is no reason to believe that the pattern of service displayed would be any different for salaried physicians. 
Table 5. 9 Comparison of Self Report (SR) and Actual Visits to a Physician by Level of BMI for GP“s and Specialist (SP) doctors within the province of NL

\begin{tabular}{|c|c|c|c|c|c|c|c|c|}
\hline & \multicolumn{2}{|c|}{ Morbidly Obese } & \multicolumn{2}{|c|}{ Obese } & \multicolumn{2}{|c|}{ Overweight } & \multicolumn{2}{|c|}{ Normal } \\
\hline & SR & Actual & SR & Actual & SR & Actual & SR & Actual \\
\hline \multirow{4}{*}{ GP } & \multicolumn{2}{|c|}{$\mathrm{n}=114$} & \multicolumn{2}{|c|}{$n=312$} & \multicolumn{2}{|c|}{$n=693$} & \multicolumn{2}{|c|}{$\mathrm{n}=717$} \\
\hline & $\mathrm{X}(\mathrm{SD})$ & $X(S D)$ & $X(S D)$ & $X(S D)$ & $X(S D)$ & $X(S D$ & $X(S D)$ & $X(S D)$ \\
\hline & $5.29(4.92)$ & $5.75(5.31)$ & $4.64(5.68)$ & $4.14(3.84)$ & $4.09(5.58)$ & $3.97(3.95)$ & $4.05(6.11)$ & $4.34(6.95)$ \\
\hline & \multicolumn{2}{|c|}{$\mathrm{n}=121$} & \multicolumn{2}{|c|}{$n=330$} & \multicolumn{2}{|c|}{$n=721$} & \multicolumn{2}{|c|}{$n=696$} \\
\hline $\mathrm{SP} *$ & $.93(2.62)$ & $3.42(3.81)$ & $.99(2.75)$ & $3.33(4.21)$ & $.73(2.10)$ & $3.22(5.08)$ & $.74(1.86)$ & $3.31(5.03)$ \\
\hline
\end{tabular}




\subsection{Poisson Prediction Models}

Five Poisson models were developed to determine the predictive relationship between level of BMI as an independent variable and the various measures of health services utilization as dependent variables. The measures of health services utilization included: (i) the expected (mean) number of actual visits to a GP and/or specialist (ii) the expected (mean) number of visits as an in-patient (iii) the total number of nights spent in hospital and (iv) the number of surgical day care procedures.

\subsubsection{Poisson Estimates for Actual Measures of Health Services Utilization}

A summary of the Poisson models are presented in tables $5.10-5.14$ (complete models are presented in Appendix J, Models 1a-1 c for GP utilization and Models 2a-2c for utilization of specialist services). For each dependent variable, three separate Poisson models were run to predict health services utilization. The models have been labeled (1), (2) and (3). Model (1) includes control variables and BMI as a categorical variable; Model (2) includes control variables and the number of chronic conditions (CC) only; and Model (3) includes the control variables, the number of chronic conditions and BMI as a categorical variable.

For each model, the parameter estimates, beta $(\beta)$, standard errors (SE) and relevant level of significance were reported. The three models were run to determine whether the number of chronic conditions acted as a mediator variable in the relationship between level of BMI and HSU. By observing the change in the $\beta$ value in the models (1) and (3), one is able to determine if level of BMI for example is working through chronic comorbidity to affect HSU. If the $\beta$ values obtained in Model (1) for any significant 
findings decreases in size when the mediating variable (i.e., number of chronic conditions) is included, the mediating variable may be affecting the relationship. The number of chronic conditions was representative of general morbidity in this sample, or Need as described in Anderson's model of Health Services Utilization. ${ }^{8-9}$

\subsubsection{Dependent Variable: Actual Number of Visits to a GP}

Poisson estimates for the relationships with the expected number of mean visits to a GP are presented in Table 5.10. Model (1) shows that being morbidly obese was predictive of the expected number of visits to a GP $(\mathrm{p}<.001)$. Model (2) indicates the increasing number of ehronic conditions was predictive of the expected number of GP services and was highly significant $(p<.001)$. These findings did not vary in Model (3). Being morbidly obese and the number of ehronic conditions were both predictive of the expected mean number of GP visits $(p<., 001)$. The number of chronic conditions partly mediates the relationship between BMI and GP visits as seen by the decrease in the $\beta$ value from .4269 to .2935

\subsubsection{Dependent Variable: Actual Number of Visits to a specialist}

Poisson estimates are presented for the predictive relationship between BMI and the expected mean numbers of actual visits to a specialist (Table 5.11). In Model (1), no significant relationship was found among BMI categories and the number of visits to a specialist. Model (2) indicates a positive significant relationship with number of chronic conditions and the expected mean number of visits to sec a specialist (p-.001). In Model (3) the findings remain unchanged. 


\subsubsection{Dependent Variable: Actual Number of Visits as an In-Patient}

Table 5.12 presents the Poisson estimates for the predictive relationship between BMI and the actual number of inpatient visits. The results of the full Poisson model are presented in Appendix K 3a-3c. Model (1) demonstrates no significant relationship between BMI category and expected mean numbers of visits as an in-patient. However, the relationship illustrated by the model is a negative one for the overweight and obese variables. Being in the overweight and obese groups compared to the normal weight group was predictive of fewer in-patient visits. Being in the morbidly obese group demonstrated a positive relationship with in-patient visits although it was not significant. Model (2) indicates a highly significant relationship between the number of chronic conditions predictive of the expected mean number of in-patient visits. As the number of chronic conditions increase, the number of visits to hospital also increases. In Model (3), the findings remain unchanged. 
Table 5.10 Poisson Regression Estimates ( $\beta$ ) and Standard Errors (SE); Dependent Variable Actual Number of Visits to a GP 1998-2003

\begin{tabular}{|c|c|c|c|c|c|c|}
\hline \multirow[t]{3}{*}{ Model } & \multicolumn{2}{|c|}{ Levels of BMI } & \multirow{2}{*}{\multicolumn{2}{|c|}{$\begin{array}{l}\text { Chronic Conditions } \\
\text { (2) }\end{array}$}} & \multirow{2}{*}{\multicolumn{2}{|c|}{$\begin{array}{l}\text { Levels of BMI and } \\
\text { Chronic Conditions } \\
\text { (3) }\end{array}$}} \\
\hline & \multicolumn{2}{|c|}{ (1) } & & & & \\
\hline & B & SE & B & SE & B & SE \\
\hline \multicolumn{7}{|l|}{$\begin{array}{l}\text { BMI } \\
\text { variables }\end{array}$} \\
\hline $25-29.9$ & .0623 & .0569 & & & .04674 & .0531 \\
\hline $30-34.9$ & -.0036 & .0691 & & & -.0580 & .0648 \\
\hline$\geq 35$ & $.4269 * * *$ & .0871 & & & $.2935 * * *$ & .0820 \\
\hline \multicolumn{7}{|l|}{$\begin{array}{l}\text { Risk } \\
\text { variable } \\
\text { \# of }\end{array}$} \\
\hline $\begin{array}{l}\text { \# of } \\
\text { chronic } \\
\text { conditions }\end{array}$ & & & $.2103 * * *$ & .0124 & $.2065^{* * *}$ & .0125 \\
\hline DF & 1644 & & 1644 & & 1644 & \\
\hline
\end{tabular}


Table 5.11 Poisson Regression Estimates $(\beta)$ and Standard Errors (SE); Dependent Variable Actual Number of Visits to a specialist Doctor 1998-2003

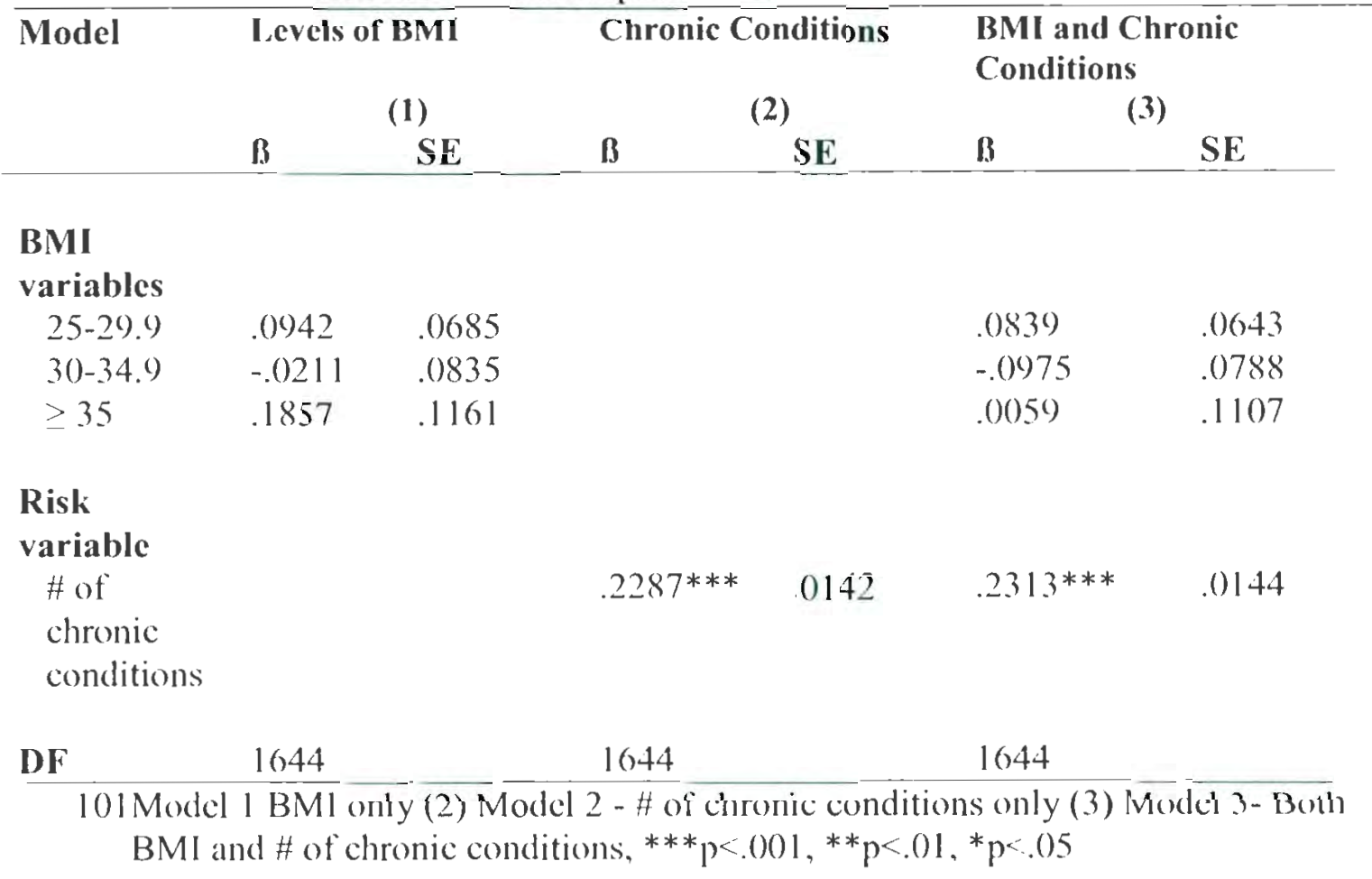


Table 5.12 Poisson Regression Estimates ( $\beta$ ) and Standard Errors (SE); Dependent Variable Actual Number of Visits as an in-Patient 1998-2003

\begin{tabular}{lll}
\hline Model Levels of BMI & Chronic Conditions & $\begin{array}{l}\text { BMII and Chronic } \\
\text { Conditions }\end{array}$
\end{tabular}

(1)

(2)

(3)

B

SE

B

SE

B

SE

\section{BMI}

variables

25-209

$-.0258 \quad .1020$

$-.1916 \quad .1272$
.2573

$-.0441$

.0992

30-34.9

$.2573 \quad .1542$

$-.2353$

.1241

$\geq 35$

.1535

.1509

Risk

variable

\# of

$.2383 * * * \quad .(1226$

$.2379 * * *$

.0227

chronic

conditions

DF

1644

1644

1644

(1) Model 1 BMI only (2) Model 2 - \# of chronic conditions only (3) Model 3- Both BMI and \# of chronic conditions, ${ }^{* * *} \mathrm{p}<.001,{ }^{* *} \mathrm{p}<.01,{ }^{*} \mathrm{p}<.05$ 


\subsubsection{Dependent variable: Total Length of Stay in Hospital}

Poisson estimates are presented for the predictive relationship between BMI category and the sum of the total numbers of days spent in hospital (Table 5.13). The output from the full model is presented in Appendix K 4a-4c. Model (1) indicates a significant negative relationship observed only between the obese group and the expected total length of stay spent in hospital $(\mathrm{p}<.001)$. Model $(2)$ indicates a positive significant relationship between the number of chronic conditions and expected total length of stay spent in hospital $(\mathrm{p}<.001)$. In Model (3) these findings remain unchanged; the number of chronic conditions but not BMI was a significant predictor of total LOS.

\subsubsection{Dependent Variable: Number of Surgical Day Care Visits}

Poisson estimates are presented for the predictive relationship between BMI category and the number of surgical day visits (Table 5.14). The details of the full output are presented in Appendix K 5a-5c. Model (1) indicates a positive significant relationship between the overweight category and the number of surgical day care visits. In Model (2) the number of chronic conditions was significantly associated with the expected number of day surgeries $(\mathrm{p}<.001)$. In Model (3), these findings do not change; the overweight category and the number of chronic conditions were significant predictors of the number of surgical day care visits. 
Table 5.13 Poisson Regression Estimates $(\beta)$ and Standard Errors (SE); Dependent Variable Total Length of Stay (LOS) in Hospital 1998-2003

\begin{tabular}{|c|c|c|c|c|c|c|}
\hline \multirow[t]{3}{*}{ Model } & \multirow{2}{*}{\multicolumn{2}{|c|}{$\begin{array}{l}\text { Levels of BMI } \\
\text { (1) }\end{array}$}} & \multirow{2}{*}{\multicolumn{2}{|c|}{$\begin{array}{c}\text { Chronic Conditions } \\
\text { (2) }\end{array}$}} & \multicolumn{2}{|c|}{$\begin{array}{l}\text { BMI and Chronic } \\
\text { Conditions }\end{array}$} \\
\hline & & & & & & \\
\hline & B & SE & B. & SE & B & SE \\
\hline \multicolumn{7}{|l|}{$\begin{array}{l}\text { BMI } \\
\text { variables }\end{array}$} \\
\hline $25-29.9$ & -.0203 & .1124 & & & -.1830 & .1090 \\
\hline $30-34.9$ & $-.6299 * * *$ & .1546 & & & $-.6490 * * *$ & .1496 \\
\hline$\geq 35$ & -.2053 & .1900 & & & -.2803 & .1851 \\
\hline
\end{tabular}

Risk

\section{variable}

\# of

$.3018 * * * \quad .0240 \quad .3059 * * *$

.0243

chronic

conditions

DF $1644 \quad 1644 \quad 1644$

101 Model 1 BMI only (2) Model 2 - \# of chronic conditions only (3) Model 3- Both BMI and \# of chronic conditions, ${ }^{* * *} p<.001,{ }^{* *} p<.01,{ }^{*} p<.05$ 
Table 5.14 Poisson Regression Estimates $(\beta)$ and Standard Errors (SE) Dependent Variable: Actual Surgical Day Care Visits 1998-2003

\begin{tabular}{|c|c|c|c|c|c|c|}
\hline \multirow[t]{3}{*}{ Model } & \multicolumn{2}{|c|}{ Levels of BMI } & \multicolumn{2}{|c|}{ Chronic Conditions } & \multicolumn{2}{|c|}{$\begin{array}{l}\text { BMI and Chronic } \\
\text { Conditions }\end{array}$} \\
\hline & \multicolumn{2}{|c|}{ (1) } & \multicolumn{2}{|c|}{ (2) } & & \\
\hline & B & SE & B & SE & $\boldsymbol{\beta}$ & SE \\
\hline \multicolumn{7}{|l|}{ BMI } \\
\hline $25-29.9$ & $.2603 *$ & .1054 & & & $.2611 *$ & .1030 \\
\hline $30-34.9$ & .2286 & .1238 & & & .1800 & .1221 \\
\hline$\geq 35$ & .0019 & .2016 & & & -.1508 & .1991 \\
\hline
\end{tabular}

\section{Risk}

variable

\# of

$.1935 * * * \quad .0235 \quad .1977 * * * \quad .0238$

chronic

conditions

DF

1644

1644

1644

(1) Model 1 BMI only (2) Model 2 - \# of chronic conditions only (3) Model 3- Both BMI and \# of chronic conditions, $*_{* *}^{*}<.001,{ }^{*} \mathrm{p}<.01,{ }^{*} \mathrm{p}<.05$ 


\subsection{Direct Costs of Obesity}

The direct costs associated with health services utilization were estimated for both physician and hospital usage over a five-year period across BMI categories. To estimate FFS physician costs, the cost per individual visit was obtained through the linkage of the 2000/2001 CCHS to the MCP physician claims database. SPSS was used for the calculations. Inflation was not adjusted for in these analyses.

Individual patient costs were summed over the five-year study period (19982003). Average cost per BMI category was calculated for each category of physician costs: (i) total physician costs, (ii) GP costs and (iii) specialist costs. The equation is as follows for each category:

$$
\text { Average Physician } \text { Cost }_{1}=\text { Total Cost }{ }_{\mathrm{BMI}} 1 / \mathrm{n}_{1}
$$

where Average Physician Cost for each category of BMI (1= normal, $2=$ overweight, $3=$ obese, 4 morbidly obese) was calculated. The Total Cost for each category of BMI was divided by the number of individuals in each category in order to determine the Average Cost for an individual in a particular category of BMI. For example, to calculate the Average Cost over the five years per individual in the obese category, the sum of Total Costs associated with visits to both GP and specialist physicians for all individuals in the obese category was calculated (i.e., $\$ 301,680$ ). The total was then divided by the number of individuals in the obese category $(n=360)$ to obtain an Average Cost of physician service per obese individual of $\$ 360.00$. For example

$$
\text { Average Physician } \text { Cost }_{3}=\text { Total Cost } \sum \mathrm{BMI}_{3} / \mathrm{n}_{3}
$$

Average Physician Cost $_{3}=\$ 301,680 / 360=\$ 838.00$ 
To estimate hospital costs, the RIW's obtained through the linkage of the 2000/2001 CCHS with the CDMS were summed over the five-year study period (1998-2003) for each individual. The total sum of individual RIW's were multiplied by the 2001 cost per weighted case or CPWC (\$4512) obtained from the CIHI. The total costs were summed for each BMI category. Average Cost per in-patient hospitalization for each category of BMI $(1=$ normal, $2=$ overweight, $3=$ obese, 4 morbidly obese $)$ was then calculated by dividing the Total cost per BMI category by the number of individuals in each category. The equation is as follows for the estimating the hospital inpatient costs for the normal weight group:

$$
\text { Average Hospital } \operatorname{Cost}_{1}=\text { Total Cost }{ }_{\text {BMI }} / \mathrm{n}_{1}
$$

where Total Cost $1=\sum \mathrm{RIW}_{1} \mathrm{X}$ CPWC

\subsubsection{Descriptive Cost Analysis}

The top portion of Table 5.15 reports the summary of physician and hospital costs by BMI category. Differences were found across all BMI categories for total FFS physician costs $(p<.05)$, costs of GP services $(p<.001)$ and costs of specialist services $(\mathrm{p}<.05)$. $\mathrm{A} \mathrm{j}$ or $\mathrm{u}$-shaped relationship was observed across the BMI categories for all physician costs. The normal and morbidly obese groups demonstrated the highest average physician costs compared to the overweight and obese groups (Figure 5.2).

Significant differences were seen across BMI categories and total hospital costs and hospital inpatient stays (Table 5.15). There were no differences across BMI categories for day care surgical costs. For total and in-patient hospital costs, a u-shaped 
relationship was demonstrated. The cost data for surgical day care across BMII levels demonstrated an inverse $\mathrm{j}$-shaped curve. The morbidly obese group and the normal weight group demonstrated the lowest average surgical day carc costs (Figure 5.3). 
Table 5.15 Summary of Average Five-Year (1998-2003) Physician and Hospital Costs by $\mathrm{BMI}$

\begin{tabular}{|c|c|c|c|c|}
\hline & \multicolumn{4}{|c|}{ BMI category } \\
\hline & $\begin{array}{l}\text { Morbid } \\
(n=127)\end{array}$ & $\begin{array}{c}\text { Obese } \\
(n=360)\end{array}$ & $\begin{array}{c}\text { Overweight } \\
(n=783)\end{array}$ & $\begin{array}{l}\text { Normal } \\
(n=766)\end{array}$ \\
\hline \multicolumn{5}{|c|}{$\begin{array}{l}\text { Physician costs in } \\
\text { dollars }{ }^{2}, \text { mean (SD) }\end{array}$} \\
\hline Total* & $1105(1241)$ & $838 \quad(1145)$ & $869 \quad(1411)$ & 9) $40 \quad(1512)$ \\
\hline $\mathrm{GP} * * *$ & $484 \quad(502)$ & $304 \quad(344)$ & $303 \quad(362)$ & $355 \quad(730)$ \\
\hline Specialist* & $621 \quad(903)$ & 533 (947) & $560 \quad(1248)$ & $584 \quad(1213)$ \\
\hline
\end{tabular}

\section{Hospital cost in}

Dollars, ${ }^{3}$ mean (SD)

\begin{tabular}{|c|c|c|c|c|}
\hline Total* & $2747(5687)$ & $1814(5011)$ & $2247(8423)$ & $2923(12648)$ \\
\hline In-patient* & $2702(5666)$ & $1615(4576)$ & $2096(8102)$ & $2777(12455)$ \\
\hline Surgical day care & $155(620)$ & $474(2768)$ & $310 \quad(2133)$ & $(1503)$ \\
\hline
\end{tabular}

TBMI categories: normal (18.5-24.0); overweight (25.0-29.9); obese (30-34.9); morbidly obese $(\geq 35 .())$.

2 Includes Fee-for-Service physicians only: all office, home, in-patient, out-paticnt. emergency consultations and all diagnostic, therapeutic procedures, in-hospital radiology and surgical procedures. Costs based on the provineial government Medical Care Plan.

${ }^{3}$ Hospital costing based on RIW costing methodology developed by the Canadian Institute for Health Information (CIHI).

${ }^{4}$ Overall sample size was $\mathrm{n}=2177$ which was divided based on BMI classification into the following categories: normal $n=817$; overweight $n=841$; obese $n-388$; morbid obese $\mathrm{n}=131$.

$* * * \mathrm{p}<.001, * * \mathrm{p}<.01, * \mathrm{p}<.05$ 


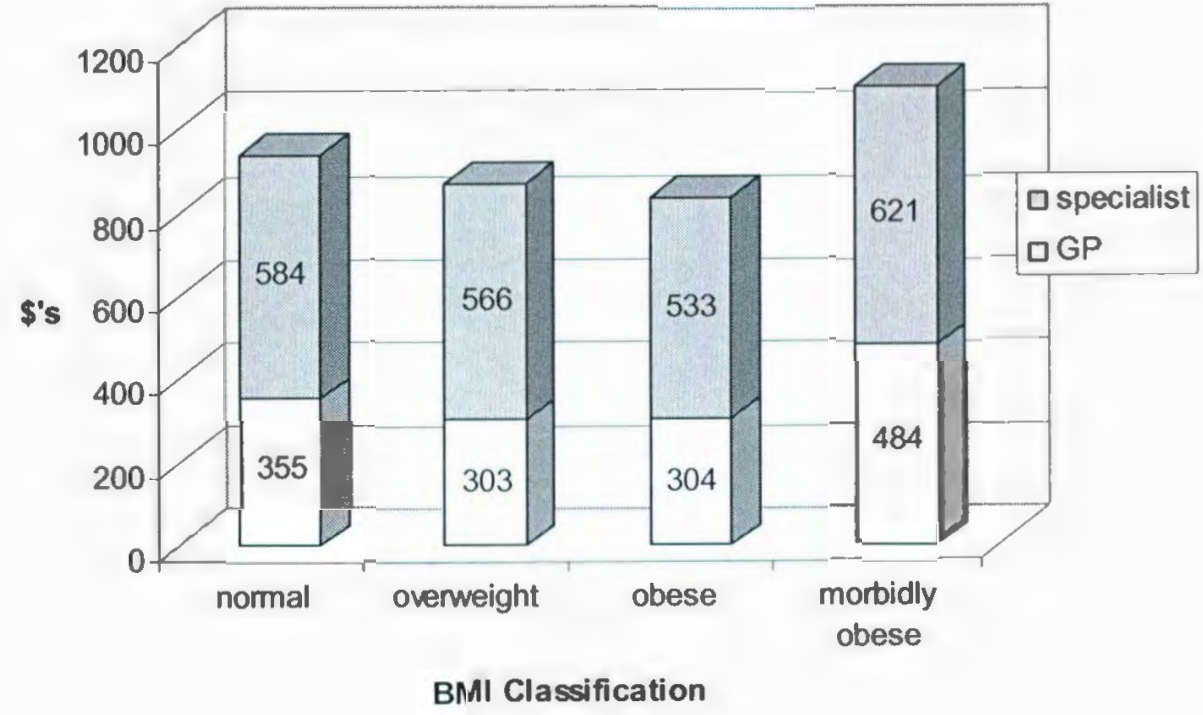

Figure 5.2 Physician Costs by Level of BMI 1998-2003 


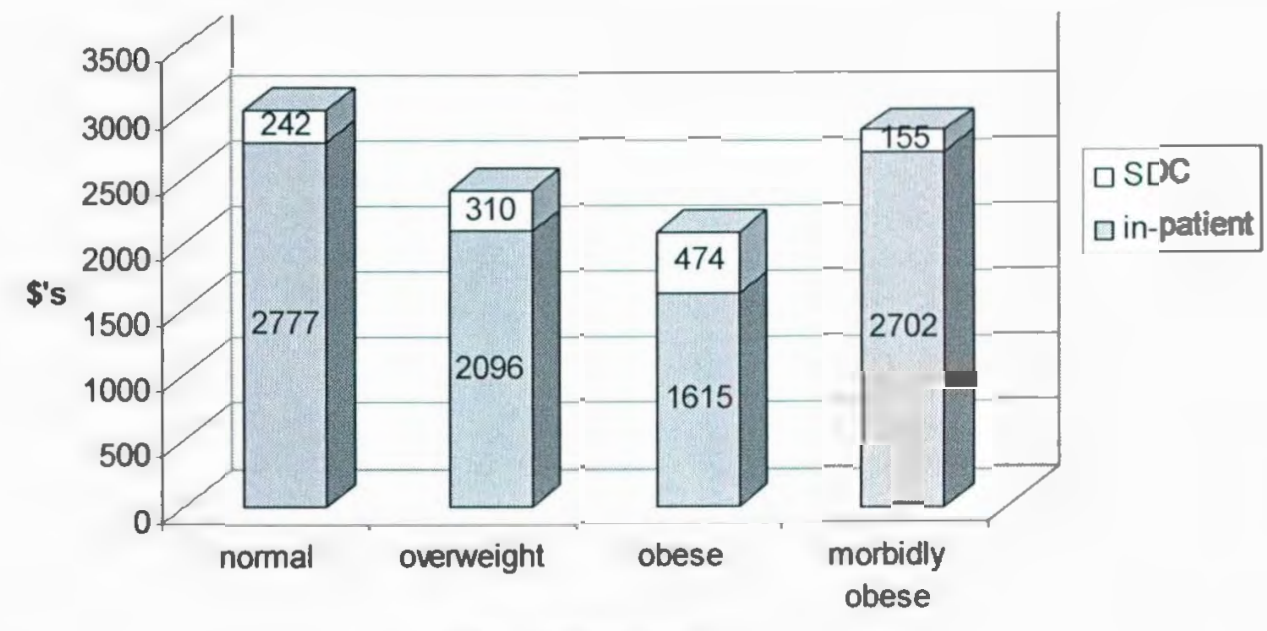

BMI Classification

Figure 5.3 Hospital in-patient Costs by Level of BMI I998-2003 


\subsubsection{Multiple Regression Cost Estimates}

Multiple regression models were developed in order to determine whether the level of BMI was predictive of physician and or hospital costs. The cost data was not normally distributed; therefore, a natural log transformation was performed producing a more normally distributed dataset. ${ }^{210}$

Two cost models were developed. In the first model, the dependent variable was total physician costs. In the second model, the dependent variable was total inpatient costs. The independent variables used in these models were the level of BMI and the control variables found to be significant having run the Poisson regression models on HSU. These control variables included: age, gender, education, region of residence and smoking behavior. The cost of day surgeries was not calculated due to the limited data on the resource-intensity-weight variable (DPG_W) associated with surgical day care use. The DPG_W variable had been coded less than a year at the time of the study.

For each dependent variable, three separate Poisson models were run to predict the costs of health services utilization. The models were labeled (1), (2) and (3). Model (1) included control variables and BMI as a categorical variable. Model (2) included control variables and the number of chronic conditions and Model (3) included the control variables, the number of chronic conditions and BMI as a categorical variable. Table 5.16 presents a summary of the results for the dependent variable: total costs of FFS physician services (see Appendix L-Models 6a-6c for complete output). Adjusting for covariates, Model (1) indicated a significant relationship between morbidly obese and 
total cost of physician services $(\mathrm{p}<.05)$. The total variance in cost explained in this model was approximately $17 \%$ indicated by the adjusted $R^{2}$ value of .1732 .

Model (2) indicated a highly significant relationship between the number of chronic conditions in the population and total physician costs $(\mathrm{p}<.001)$. The number of chronic conditions and the control variables explained $26 \%$ of the variance in total physician costs (adjusted $R^{2}=.259$ ). In Model (3), although the morbidly obese category remained a significant predictor of total physician costs, its significance decreased $(\mathrm{p}<.01)$, shown by the change in the $\beta$ from .4329 to .2827 . The number of chronic conditions in the population remained a highly significant predictor of total physician costs $(\mathrm{p}<.001)$. Model (3) suggests it is the number of chronic conditions that is predictive of total physician costs as the adjusted $R^{2}$ value did not vary in this model Overall, the complete set of variables in the model demonstrated low predictability of total physician costs.

A summary of the multiple regression estimates for total in-patient hospital costs are presented in Table 5.17 (Appendix L Model 7a-7c). Model (1) indicated there was no predictive relationship between BMI and total in-patient costs. In Model (2), although there was a significant relationship between the number of chronic conditions and inpatient costs $(\mathrm{p}<.001)$, the explained variance was only $6 \%$ (adjusted $\left.\mathrm{R}^{2} .0648\right)$. In Model (3), BMI was not a significant predictor of total in-patient costs but the number of chronic conditions remained significant ( $p<.001)$. The explained variance of Model (3) increased slightly to $7 \%$ (adjusted $\mathrm{R}^{2}=.0710$ ). The complete set of variables in this model demonstrated very low predictability for total in-patient costs. 
Table 5.16 Multiple Regression Estimates ( $\beta$ ) and Standard Errors (SE) Dependent Variable: Total Costs of Physician Services 1998-2003

\begin{tabular}{|c|c|c|c|c|c|c|c|c|c|}
\hline \multirow[t]{2}{*}{ Model } & \multicolumn{2}{|c|}{$\begin{array}{l}\text { Levels of BMI } \\
\text { (I) }\end{array}$} & \multicolumn{4}{|c|}{$\begin{array}{l}\text { Chronic } \\
\text { Conditions } \\
\text { (2) }\end{array}$} & \multicolumn{3}{|c|}{$\begin{array}{l}\text { BMI and Chronic } \\
\text { Conditions } \\
\text { (3) }\end{array}$} \\
\hline & B & SE & $t$ & B & SE & $t$ & B & SE & $t$ \\
\hline $\begin{array}{l}\text { BMI } \\
\text { variables } \\
25-29.9 \\
30-34.9 \\
\geq 35\end{array}$ & $\begin{array}{l}.0437 \\
.0666 \\
.4392 * *\end{array}$ & $\begin{array}{l}.0775 \\
.0932 \\
.1397\end{array}$ & $\begin{array}{l}.564 \\
.715 \\
3.145\end{array}$ & & & & $\begin{array}{l}.0413 \\
-.0(0) 15 \\
.2827^{*}\end{array}$ & $\begin{array}{l}.0734 \\
.0883 \\
.1327\end{array}$ & $\begin{array}{l}.563 \\
-.017 \\
2.131\end{array}$ \\
\hline $\begin{array}{l}\text { Risk } \\
\text { variable } \\
\text { \# of } \\
\text { CC's }^{\prime}\end{array}$ & & & & $.3029 * * *$ & .0223 & 13.549 & $.2993 * * *$ & .0225 & 13.323 \\
\hline DF & 1507 & & & 1509 & & & 1506 & & \\
\hline $\begin{array}{l}\mathbf{R}^{2} \\
\text { Adjusted } \\
\mathbf{R}^{2}\end{array}$ & $\begin{array}{l}.1808 \\
.1732\end{array}$ & & & $\begin{array}{l}.2648 \\
.2590\end{array}$ & & & $\begin{array}{l}.2672 \\
.2599\end{array}$ & & \\
\hline $\begin{array}{l}\text { F- } \\
\text { statistic }\end{array}$ & 23.76 & & & 45.3 & & & 36.61 & & \\
\hline
\end{tabular}

101 Model 1 BMl only (2) Model 2 - \# of chronic conditions only (3) Model 3- Both $\mathrm{BMI}$ and \# of chronic conditions, ${ }^{* * *} \mathrm{p}<.0(0),{ }^{*} * \mathrm{p}<.01, * \mathrm{p} .05$ 
Table 5.17 Multiple Regression Estimates ( $\beta$ ) and Standard Errors (SE) Dependent Variable: Total Costs for in-Patient Hospital Services 1998-2003

\begin{tabular}{|c|c|c|c|c|c|c|c|c|c|}
\hline \multirow[t]{2}{*}{ Model } & \multicolumn{2}{|c|}{$\begin{array}{c}\text { Levels of BMI } \\
\text { (1) }\end{array}$} & \multirow[b]{2}{*}{$\mathbf{t}$} & \multicolumn{2}{|c|}{$\begin{array}{l}\text { Chronic } \\
\text { Conditions } \\
\qquad(2)\end{array}$} & \multirow[b]{2}{*}{$t$} & \multicolumn{2}{|c|}{$\begin{array}{l}\text { BMI and Chronic } \\
\text { Conditions } \\
\text { (3) }\end{array}$} & \multirow[b]{2}{*}{$\mathbf{t}$} \\
\hline & $\mathbf{B}$ & SE & & $\mathbf{B}$ & SE & & B & SE & \\
\hline $\begin{array}{l}\text { BMI } \\
\text { variable } \\
\text { s }\end{array}$ & & & & & & & & & \\
\hline $25-29.9$ & .0114 & .0968 & .117 & & & & .0001 & .0945 & .002 \\
\hline $30-34.9$ & -.1989 & .1203 & -.16 .53 & & & & -.2270 & .1176 & $\begin{array}{l}- \\
1.93\end{array}$ \\
\hline$\geq 35$ & .1784 & .1505 & 1.185 & & & & .1176 & .1475 & .797 \\
\hline $\begin{array}{l}\text { Risk } \\
\text { variable }\end{array}$ & & & & & & & & & \\
\hline $\begin{array}{l}\text { \# of } \\
\mathrm{CC}^{\circ} \mathrm{s}\end{array}$ & & & & $.1130 * * *$ & .0241 & 4.698 & $.1131 * * *$ & .0240 & 4.70 \\
\hline DF & 429 & & & 431 & & & 428 & & \\
\hline $\mathbf{R}^{2}$ & .0563 & & & .0902 & & & .1025 & & \\
\hline $\begin{array}{l}\text { Adjuste } \\
\text { d } R^{2}\end{array}$ & .0255 & & & .0648 & & & .0710 & & \\
\hline $\begin{array}{l}\text { F- } \\
\text { statistic }\end{array}$ & 1.826 & & & 3.559 & & & 3.258 & & \\
\hline
\end{tabular}




\section{Chapter 6 Discussion and Conclusions}

This chapter discusses the key findings of the study and draws conclusions from the analysis and the findings. As well the strengths and limitations of the study design and recommendations for future research are presented. Policy implications and recommendations for the treatment and prevention of obesity are discussed briefly.

\subsection{Summary of Key Findings}

In the health survey, $6 \%$ and $17 \%$ of the study sample were classified as morbidly obese and obese and 39\% and 37\% were classified as overweight and normal weight, respectively. Analyses of the prevalence of chronic diseases reported by individuals suggest that a monotonic relationship exists between increasing level of BMI and the likelihood of having been diagnosed with some form of chronic disease. Of the 2177 linked survey respondents, $94 \%$ had one or more visits with a physician, and $25 \%$ had either one hospital in-patient stay or one surgical day care visit over the study period. The morbidly obese group had a significantly higher median number of GP visits compared to other BMI categories. This finding was repeated in the estimation of the crude utilization rate of total physician visits across levels of BMI, where the morbidly obese group demonstrated a significantly higher projected utilization rate of total physician visits. The data on physician utilization and costs illustrated a $\mathrm{j}$ - shaped curve across levels of BMI. The Poisson regression models demonstrated the morbidly obese group to be the only independent BMI category predicting the expected mean number of visits to a GP, having controlled for the number of chronic conditions and other variables. The predictive value of being morbidly obese decreased when the number of chronic conditions was added to 
the model, however it did remain an independent significant predictor. The number of chronic conditions was a highly significant predictor of GP utilization in all three models, suggesting that level of morbidity defined as a Need variable in Anderson's Model of Health Services Utilization was the main predictor of physician utilization in this analysis.

Body mass index was not a predictor of any measure of hospitalization. However, the number of chronic conditions reported by individuals was a significant predictor of all measures of hospitalization. The obese category showed a significant negative relationship with LOS, demonstrating a $\mathrm{j}$ - shaped curve. Being overweight was a significant predictor of increased surgical day care visits. Interestingly, the relationship between the level of BMI and surgical day care visits was an inverse $\mathrm{j}$ or $\mathrm{u}$-shaped relationship. One interpretation of this finding may be that surgical day care is utilized more often by individuals considered to be at low risk for complications compared to those individuals at high risk.

There were significant differences in the costs of physician services. The cost of treating morbidly obese individuals compared with normal weight individuals was $36 \%$ and $6 \%$ higher for GP and specialist healthcare services, respectively. Being morbidly obese was a significant predictor of total physician costs when the number of chronic conditions and other variables were controlled for in the multiple regression models. There were significant differences in the costs of in-patient hospital services, however the relationship was surprising. The cost of treating the normal weight category was significantly higher than the other BMI categories. The level of BMl was not predictive 
of in-patient costs when the number of chronic conditions and other variables were controlled for in the multiple regression models.

\subsubsection{The 2000/2001 Canadian Community Health Survey}

Compared to the normal weight group, analyzing associations between the categories of obesity, lifestyle and health status variables demonstrated individuals with a $\mathrm{BMI} \geq 30$ and $\mathrm{BMI} \geq 35$ were more likely to be inactive and consume $<5$ servings of fruits/vegetables per day (Table 5.2). These findings are cross-sectional in nature, and therefore it is difficult to ascertain whether it is the decrease in activity that has caused the current increase in obesity or whether the increase in obesity has caused a decrease in physical activity due to its association with comorbid conditions such as hypertension and type II diabetes. Some researchers support the theory a decrease in Energy out $_{\text {tas }}$ caused the recent increase in body weight, ${ }^{176,182,185-186}$ while others support the theory that the increase in obesity has been caused by an increase in Energy ${ }_{\text {in. }}{ }^{178-181}$ Morbidly obese individuals were less likely to report incomes in the high income bracket. The obese group was more likely to report having secondary or $<$ secondary education. Although not always consistent, some researchers have reported an inverse relationship between obesity and socioeconomic status which includes level of education, income and occupation. ${ }^{137,169,188}$ Morbid obesity, obesity and overweight individuals compared to normal weight individuals reported a higher prevalence of chronic diseases such as cerebro/cardiovascular diseases, hypertension, and endocrine diseases. The logistic regression models, controlling for age and sex, demonstrated a positive relationship across increasing levels of BMI and the increasing likelihood of having been diagnosed 
with several chronic diseases (Tables 5.3, 5.6). Other studies have indicated similar doseresponse relationships between obesity and coronary heart disease, cardiovascular disease, hypertension and diabetes. ${ }^{21,94,130}$

\subsubsection{Generalizability of Findings}

The analysis comparing self reported and actual GP visits at a provincial elvel reported no significant differences with the exception of the northern region where the self reported number of GP visits were significantly higher than the actual visits obtained for the morbidly obese group only. However as the regression results showed a significant predictive relationship between the morbidly obese and GP visits, it was agreed that the potential missing data of some visits in this region did not pose a problem. There were significant differences between self reported and actual visits to a specialist, however due to the small number of visits provided in the self reported data and that the utilization patterns were similar across levels of BMI and both datasets, the decision was made to use the actual data on specialist visits in order to provide an increased number of outcomes. Approximately $62 \%$ of the specialists in NL were paid FFS in 2000 there is no known reason to believe the patterns reported in the current analysis across levels of BMI would be any different if data for visits to salaried specialists were included. It is suggested that the shape of the relationship would be similar without other evidence.

\subsubsection{Obesity and Actual Visits to a Primary Care Provider}

When compared to the normal weight, the overweight and obese groups, the morbidly obese group demonstrated a significantly higher median number of both self reported and actual visits to a GP (Tables 5.4 and 5.7). Several studies have suggested a 
link between obesity defined as $\mathrm{BMI} \geq 30$ and increased utilization of physician services. ${ }^{38,72-73}$ Most studies analyzed obesity as an inclusive category, without analyzing the obese sub-groups. The number of GP visits stratified by BMI illustrated a j-shaped relationship. It has been suggested that overweight and obese individuals especially women are less likely to avail of preventive health services such as cervical smear and mammography. It may be that the normal weight groups avail of these and other preventive health services such as a medical check-up more often than other BMI groups. ${ }^{72}$ The present study found morbid obesity controlling for known confounders such as age, gender, region of residence, smoking, level of education and physical activity was a significant predictor of objective GP services (Table 5.10). Similar findings have been reported in other studies; even though variable definitions of obesity have been used. Given the well known association between excessive body weight and the increased likelihood of chronic diseases, it would seem logical that obese individuals with a high level of morbidity would demonstrate higher utilization of some healthcare services. Most studies identified in the literature review used the current definition of obesity (BMI $\geq 30 \mathrm{~kg} / \mathrm{m}^{2}$ ) encompassing all sub-categories of obesity and do not for example separate out the other categories of excessive overweight such as obesity class I $\left(\mathrm{BMI} \geq 30 \mathrm{~kg} / \mathrm{m}^{2}\right)$, obesity class II $\left(\mathrm{BMI} \geq 35 \mathrm{~kg} / \mathrm{m}^{2}\right)$ and obesity class III $(\mathrm{BMI} \geq 40$ $\left.\mathrm{kg} / \mathrm{m}^{2}\right) \cdot{ }^{32-33,72-73}$ In contrast to the current study, another study controlling for sex, smoking status, alcohol intake, household income, ethnic group, and education found no significant relationship between $\mathrm{BMI}$ and increased GP utilization. ${ }^{35} \mathrm{~A}$ potential explanation for this null finding may have been the small number of HSU outcome 
measures available as questions on GP utilization were limited to a two week recall period compared with other studies that often used 12 month recall period. As well the age-range of the study sample was limited from 50 to 69 years of age. In a study conducted in Germany including 2301 women between 25 and 74 years of age, the authors found no significant relationship between obese women $\left(\mathrm{BMI} \geq 30 \mathrm{~kg} / \mathrm{m}^{2}\right)$ and total physician visits or hospitalizations. ${ }^{72}$

Regression analysis demonstrated being morbidly obese remained an independent predictor of GP utilization controlling for the presence of chronic disease, although its impact on the outcome was attenuated by the inclusion of the number of chronic diseases (Table 5.10). Some studies using regression analysis to study the relationship between BMI and HSU choose not to control for obesity associated comorbidites on the basis that these conditions lie on a causal pathway with HSU and thus controlling for them constitutes over adjustment. ${ }^{33,38,72}$

\subsubsection{Obesity and Hospitalization}

There were no significant relationships found between level of obesity and any of the measures of hospital utilization (Tables $5.11-5.14$ ). Several studies have reported similar findings, ${ }^{31-32,35,38}$ however other studies reported findings in contrast to the present one. ${ }^{33-34,36,41,45,73-74}$ In a large study $(n=8762)$ authors found the number of inpatient days increased with each increasing BMI category. ${ }^{45}$ Controlling for age, race, insurance status, marital status, education, family income, region, smoking behavior and drinking behavior, significant relationships were also found between obesity and the number of inpatient days. ${ }^{33} \mathrm{~A}$ large study $(\mathrm{n}=8754)$ found mild obesity defined as a BMI 
$\geq 30$ and moderate to severe obesity defined as a $\mathrm{BMI} \geq 35$ to be related to a higher risk of hospitalization in individuals 65 to 75 years of age. ${ }^{34}$ A significant relationship was found between obesity and increased in-patient days. Potential contributing factors were the large sample size $(n=17,118)$ and the extended age range of 20 years of age and over. ${ }^{41}$ A study examined the likelihood of an overnight stay in hospital in the previous 12 months and its associated LOS and its relationship with BMI in a large national survey $(n=113603)$ and was published in Canada in 2006. The authors reported that obesity defined as $\mathrm{BMI} \geq 30$, was associated with the increased likelihood of being an in-patient and was a significant predictor for most categories of LOS. ${ }^{46}$ Given the extensive literature published on obesity and its associated adverse health consequences, it is surprising that this study as well as others did not find obesity to be a predictor of hospitalization. There are several possible explanations for this study's null finding. Although the survey sample size for the current study was 2345 individuals, the sub group analysis based on the WHO BMI categories reduced the sample for the obese and morbidly obese group to 407 and 141, respectively. These smaller sample sizes combined with an outcome that was relatively rare (i.e., only $25 \%$ of the sample had experienced any type of hospitalization visit) may have reduced the power of the study, the chance of finding a significant result. The common methodological difference across many of these positive studies appears to be the large sample size allowing for an increased number of outcomes of interest such as hospitalization to occur. As well many of the chronic conditions that obese people suffer from may not become acute or require hospital care until the individuals are well into their 60 's or older. This study analyzed health survey 
data from individuals between the ages of 20 and 64 years of age. Studying an older age group might allow for ascertainment of more outcome measures of interest. Also self reported BMI on its own may not be a valid measure of morbidity. Although BMI correlates highly with excess body fat, this measure provided no indication of the location of excess adiposity (e.g., abdominal obesity) which researchers suggest is an important indicator of increased health risk and should be measured in conjunction with heights and weights. ${ }^{70,94}$ It may be that the null findings demonstrated by the analysis of BMI and the use of hospital services were correct and obese individuals compared to other BMI categories were no more likely to be users or be predictors of hospital services. ${ }^{212}$

\subsubsection{Obesity and Andersen's Model of Health Services Utilization}

There were no studies reviewed that used Andersen's model to guide, analyze or interpret the relationship between BMI and health services utilization. The use of this model in the current thesis highlights an important finding that may have policy implications. Although the logistic regression analysis demonstrated that increasing levels of BMI predisposed an individual to the increased likelihood of chronic illness, the Poisson regression analysis suggested that it was mainly the presence of chronic disease on its own that increased the need for healthcare services as a Health Behavior, and with the exception of being morbidly obese, the other levels of BMI did not increase healthcare utilization directly. As a result if the pertinent driver of use of health services is primarily the presence of chronic disease, it may be that health policy needs to be directed firstly to the management, treatment and prevention of chronic conditions in the 
population and secondly to risk factors for the development of chronic disease such as excessive overweight, smoking and sedentary behaviors.

\subsubsection{Obesity and Direct Healthcare Costs}

Due to the increasing prevalence of obesity world-wide, numerous studies have been undertaken in order to capture obesity associated healthcare costs. Most of these studies have used a COI methodology. As previously discussed in detail in Chapter 2, the COI prevalence-based approach is the most common method for estimating costs or burden of illness. This approach uses the top-down method by calculating the Population Attributable Risk Fraction (PAF \%). Most empirical studies have used this approach and reported the direct health care costs attributable to obesity representing approximately between $2 \%$ and $7 \%$ of total healthcare expenditures. ${ }^{33,36,40-48,142}$

Although COI estimates assist in determining medical research priorities, there are some key limitations associated with this approach: different BMI cut-offs have been used to define obesity in different countries and the inclusion of different obesity-related medical conditions will impact on the calculated costs.

Few studies using individual-level data linked to healthcare databases have estimated the impact of obesity on HSU. ${ }^{40-42,45}$ This type of analysis however, can provide useful information for healthcare providers. The current study utilized individuallevel data to examine the relationship between $\mathrm{BMI}$ and direct healthcare costs for both physician and inpatient hospital costs. An advantage of using this approach is that specific factors known to have an impact on HSU such as age, gender and education can be controlled for in the statistical analysis. 
Health care costs are an extension of the utilization patterns and can serve as a proxy for HSU. The findings from the cost analysis in the current study are similar to those on HSU. For example, utilization of GP services and the associated costs for the morbidly obese group were much higher compared with the normal weight group, as cost is estimated by these two factors: utilization and the service cost.

Overall the morbidly obese group had total physician costs that were $17 \%$ higher than those of the normal weight group. The morbidly obese group was associated with $36 \%$ and $6 \%$ higher costs of GP and specialist services, respectively (Table 5.15). These findings are similar to those in other studies reporting average increases in healthcare expenditures between $31 \%$ and $37 \%$ when comparing obese with normal weight groups, even though different data sets were examined and varying methodological approaches were utilized. ${ }^{41,45,73}$ One author reported a large increase of $61 \%$ in total healthcare expenditures when comparing obese and non obese patients. A potential explanation for the high cost of physician utilization may be due to the study sample. As compared to many studies using population health surveys to obtain information on healthcare utilization and costs, this author examined primary care costs associated with newly registered adult patients randomly allocated to physicians. ${ }^{38}$ One interesting finding was that there were no differences in specialist visits across BMI categories but there were significant differences in costs. Being morbidly obese had significantly higher costs for specialist services (e.g., \$621 versus $\$ 583$ for obese, $\$ 566$ for overweight and $\$ 584$ for normal weight). It may be that morbidly obese individuals have a higher number of 
chronic conditions and that their health needs are more complex and require more costly treatment. $^{21}$

Morbid obesity remained a significant predictor of total physician costs adjusting for other control variables which is supported in the literature (Table 5.16). ${ }^{72-73}$ Physician costs are directly linked to utilization of physicians, and therefore this finding was expected. Consistent with the earlier findings on utilization, morbid obesity remained an independent significant predictor for total physician costs controlling for known confounders and the number of chronic conditions (Table 5.16, Model 3). A similar finding in another study reported that obesity was significantly associated with increased primary care costs controlling for physical health as a proxy for comorbidity, age, education income, sex, smoking, drinking and mental health status. ${ }^{38}$

There were no significant associations found between morbid obesity and total hospital costs, consistent with the findings on hospital utilization supported by other studies. ${ }^{40,42}$ A study on a large representative sample of the US population $(n=16,217)$ was conducted. The high BMI category defined as BMI $>36$ was not associated with increased total healthcare expenditures such as inpatient, outpatient and prescriptions costs. $^{34}$ In contrast, two larger studies $(n=16,262, n=33,196)$, found significant relationships between obesity and increased overall healthcare expenditures controlling for covariates. ${ }^{217-218}$

The results from this study were fairly consistent with previous studies and reinforce the credibility of the results, as many of the studies discussed were conducted in different healthcare systems, with different conditions of access and service. Many of the 
prediction studies suggest that the relationship between obesity and health services utilization and or associated costs are a function of treating obesity related medical conditions and that BMI on its own is not an independent risk factor for measures of utilization. However, in the present study, morbid obesity remained an independent significant predictor of GP visits and total physician costs, controlling for known confounders and the number of chronic conditions. It may be that morbidly obese individuals, even without having been diagnosed with chronic conditions see themselves as unhealthy individuals and frequent the physician more frequently. It has been suggested that the relationship observed between obesity and GP utilization is associated with divergent patient health beliefs and help-seeking behavior or supplier induced demand. Either obese patients believe they are at increased risk for health problems that may be prevented or attenuated by more frequent visits to their primary care providers, ${ }^{38}$ or physicians may be cognizant of the increased risk associated with obesity and may request more follow-up appointments with diagnostic testing and monitoring. The increased utilization of GP services may occur in the absence of actual health status differences between obese and non obese groups. This behavior may be motivated by perceived risk for potential health problems by either the individual or the physician.

\subsection{Conclusions}

The main purpose of this study was to explore the relationship between BMI and its association with HSU and direct healthcare costs by examining the relationship between obesity and the presence of chronic diseases. Based on the data analysis and study findings, the following conclusions have been reached. 
The morbidly obese and obese groups were significantly more likely to report having been diagnosed with a number of chronic diseases, primarily those related to cerebro/cardiovascular diseases, hypertension and diabetes. Morbid obesity was an independent predictor of increased visits to a GP, and with the addition of the number of chronic conditions attenuating the relationship, morbid obesity remained a significant independent predictor of this service. Increasing level of BMI was not found to be associated with any measure of hospital utilization, although the relationship remained $\mathrm{j}$ shaped. Controlling for covariates and the number of chronic diseases, morbid obesity remained a significant independent predictor of total physician costs. Morbid obesity was not found to be a predictor of hospital costs. Overall it was interesting and surprising that the $\mathrm{j}$-shaped relationship between BMI and HSU found in this study was similar to the published j-shaped relationship found between BMI and increased risk of morbidity and mortality that has been extensively documented. ${ }^{21}$ The relationship between BMI and morbidity and health services utilization appears to be non-linear in nature and does not follow the straight line dose-response relationship that might be expected. This infers a more complex relationship between excess body fat, health and utilization of health services.

\subsection{Strengths and Limitations}

There are a number of strengths associated with this study design. The study sample is representative of the NL population at a provincial level. The health survey and administrative databases provided a rich dataset of variables that were used in the analysis of the relationships between obesity and health services utilization and 
associated direct health care costs. As a result, variables known to have an impact on health services utilization such as age, gender and smoking were controlled for in statistical analysis and the impact of $\mathrm{BMI}$ on health services utilization was explored independently. Due to the use of individual-level data, this study represents a more correct relationship between BMI and health services utilization. The linkage of several datasets was conducted and objective health services utilization data were obtained. This allowed for more complex analyses of data over time and the ability to look at level of BMI as a predictor of health services utilization.

The study used individual-level data to estimate the direct costs of obesity. This approach allowed for the calculation of direct costs per patient and per individual level of BMI. This is in comparison to many of the published studies utilizing the prevalencebased COI approach. The utilization and costs of FFS physicians were assumed to be complete and accurate as this information is in claims database and is used in the payment of FFS physicians. In addition hospital costs were based on CIHI's costing methodology using both resource-intensity-weights (i.e., proxies statistically derived, using empirical data based on patient diagnosis or procedure, illness severity, comorbidity and age) and the cost per weighted case as per the CIHI published cost data and not an average cost per hospital day.

There are several limitations associated with this study. The sample population was limited to those individuals between the ages of 20 to 64 years of age with a self reported BMI. As many of the chronic conditions associated with obesity are not 
diagnosed until later in life, extending the age of the sample may have produced a different set of results.

In addition, the power of the study may have been low for the study of hospital admissions as an outcome variable. While the eligible study sample size was 2345 , the research design required the sample be categorized into levels of BMI. The numbers in the obese categories were relatively small. Using small numbers associated with rare events (i.e., hospital visits) may have resulted in low power. Although, the numbers in this study were large enough to run the relevant statistical tests, a larger sample may have increased the power of the study. There was a large survey of the adult NL population ( $\mathrm{n}$ $>10,000)$ conducted in 1995 examining HSU. These survey respondents provided consent for the use of their individual health insurance numbers in order to examine individual health utilization patterns until 1999. Obtaining permission from these survey respondents to examine HSU was not considered feasible for the current research. ${ }^{212}$

The use of cross-sectional survey data limits the ability to draw causal inferences about relationships. Analysis of the survey data is limited to a discussion of associations with obesity. In addition, the self reported health survey responses should be interpreted with caution, even though the response rate for the survey was approximately $84 \%$. Self reported information often suffers from information bias in terms of recall, social desirability and misclassification bias. Issues of recall bias may impact on survey responses, as many survey respondents do not always remember past behaviours or experiences accurately. Social desirability bias is providing responses the interviewer would like to hear. This bias relates to questions primarily on lifestyle such as level of 
physical activity, smoking, drinking habits, food intake and health status. Survey respondents often provide answers to these types of questions considered to be acceptable to society. In this study, BMI was calculated using self reported heights which may be overestimated and weights which are often underestimated, impacting on an individual's weight classification and may result in misclassification of the BMI. It is generally agreed that this systematic bias may result in conservative study findings.

\subsection{Future Research}

This was one of few studies in Canada using individual-level data linked to longitudinal datasets to model the relationship between the level of BMI, health services utilization and direct healthcare costs. It would be interesting to examine whether the findings in this study are similar to findings in alternative datasets in other provinces, or in healthcare systems such as in the UK and Australia. This population sample may serve as a baseline for a longitudinal study on future health services utilization patterns and costs, as well as on mortality rates and causes of death.

In this study $95 \%$ of individuals visited a physician annually, however only $25 \%$ of individuals had any type of hospitalization. To study hospital utilization as an outcome, either a larger population sample or a longer time period are needed to ensure adequate power of the study.

There is a high level of morbidity associated with obesity in the NL population. As indicated by the published COI literature, the indirect health care costs associated with obesity are considerable. It would be beneficial in a future study to include indirect costs 
for example loss of productivity, work days lost and intangible costs to the individual such as the impact on social status, self-esteem, income and educational attainment.

The current study did not include drug utilization or cost of medications associated with obesity. Many of the studies have reviewed this relationship. These studies analyzed levels of BMI and drug utilization patterns often concluding that obese individuals when compared to normal weight groups were more likely to be prescribed medications such as anti-hypertensive and cholesterol lowering drugs. With the development of the Pharmacy Network in NL, a future study exploring this relationship will soon be possible. Finally as the results of the present study demonstrated, a nonlinear relationship existed between level of BMI and many measures of health services utilization illustrated by the $\mathrm{j}$ or $\mathrm{u}$-shaped curve. More research is needed to better understand the complex relationship between excess body fat, increased health risk and its association with health services utilization.

\subsection{Policy Implications}

In this section, the policy implications will be briefly discussed from the perspectives of the obese individual using the healthcare system and from a public health prevention approach. In the current study increasing BMI was associated with increasing prevalence of chronic disease. Interventions focused on the treatment, management and prevention of chronic disease or obesity as a risk factor are very similar in nature with interventions aimed at targeting high risk individuals and implementing population health approaches. ${ }^{2}$ The prevalence of chronic diseases in particular those related to cardiovascular illness, diabetes, cancers and respiratory diseases are increasing in both 
developed and under developed countries and are being described by some as the 'world's next tidal wave' or 'tsunami' due to the numbers being affected and the devastating impact on quality of life, morbidity and mortality. ${ }^{219}$ As discussed in Chapter 1, the main risk factors for the development of chronic diseases include high blood pressure, high cholesterol, excessive overweight or obesity, low fruit and vegetable intake, physical inactivity and tobacco use. ${ }^{2}$ The focus of the current study has been on excessive overweight as a risk factor for chronic disease and increased health services utilization and therefore the policy interventions will focus on the current study findings.

In the current study, the morbidly obese group represented $6 \%$ of the population studied and when compared to other BMI categories were more likely to utilize GP services. The morbidly obese group demonstrated significantly higher total physician costs when compared to the other BMI categories and remained a significant predictor of both GP visits and total physician costs in multivariate models controlling for covariates including the number of chronic diseases. Although the morbidly obese were more likely to utilize certain services, this group represented only $6 \%$ of the population studied.

Therefore, it might appear to be a minimal threat to the current healthcare system in terms of overall use and cost. Interpretation of these findings should be exercised with caution due to the short follow-up time for the exploration of some study outcomes and the emerging epidemic of childhood obesity. Although the long-term effects of excess body weight have not been examined in the current study, this population may serve as a baseline for future longitudinal studies on health services utilization and associated costs. One challenge to this approach may be that health services utilization of high risk 
populations may change over time if diagnosed and targeted with more intensive treatment due to increased awareness by physicians and or other health professionals or if public efforts are successful in promoting healthy body weights.

\subsubsection{Individual-Based Approach}

Based on the current study's finding concerted therapeutic efforts may be focused on the morbidly obese group, the BMI category found to be associated with increased utilization and costs in the healthcare system. Traditionally, efforts have been made by health professionals to reduce a patient's excess body weight associated comorbidities. Efforts have included: pharmacologic, reduced calorie diets, surgery for the excessively obese and psychotherapy, as well as behavioral therapy in an attempt to change lifestyle habits. The management of obesity from a health professional's point of view has been to manage the associated chronic diseases such as hypertension, diabetes or high cholesterol. Very recently, in Canada clinical practice guidelines were published on the management and prevention of obesity in adults and children. These guidelines are based

on the most up-to-date evidence of treating and managing obesity. ${ }^{220}$ Using these guidelines, an example of an individual strategy to address the treatment and management of obesity is discussed.

\subsubsection{Individual-Based Intervention}

In 2007, the Canadian Medical Association Journal published a supplement on Canadian clinical practice guidelines related to the management and prevention of obesity in adults and children. ${ }^{220}$ The supplement is the summary of a detailed report written by a panel of experts convened by Obesity Canada, a non-for-profit organization 
founded in 1999 to improve the health of Canadians by decreasing the occurrence of obesity. This report highlights the management and treatment options available for overweight and or obese adults based on the most recent peer-reviewed evidence for lifestyle modifications, available pharmacotherapy and surgery options. This report identifies an algorithm for the assessment and stepwise management of an overweight or obese adult. The treatment options for an individual with a BMI $\geq 35 \mathrm{~kg} / \mathrm{m}^{2}$ recommended for application by a health professional are presented below. If the health professional (often the primary care physician) is concerned that a patient is obese or at risk for obesity-related diseases, the following should be conducted. The BMI and waist circumference should be measured and if either is greater than the recommended cutpoints, clinical and laboratory investigations should be conducted in order to assess comorbidities (e.g., blood pressure, heart rate, fasting glucose, lipid profile). Following the clinical assessment the patient should be screened for depression, eating and or mood disorders. While comorbidties, should be treated according to normal standards of care, the patient should be assessed for readiness to change lifestyle behaviors. ${ }^{220}$

According to the algorithm, the next step for the primary healthcare provider is to help establish goals and a lifestyle modification program for weight loss with the advice and consultation of several health professionals. In order to achieve this, the clinical practice guidelines suggest it is valuable to have a 'Health Team' develop and advise the patient on a lifestyle modification program to include components on nutrition intake, physical activity and participating in cognitive-behavior therapy. This approach requires input from a multidisciplinary team of health professionals including a GP and or 
specialist, nutrition health professional, exercise health professional, clinical psychologist, and long-term monitoring as is the case for many chronic diseases such as type II diabetes. During follow-up period, an on-going evaluation should take place to determine progress. If satisfactory progress is being accomplished then monitoring continues, and if progress is not successful then other options such as the introduction of pharmacotherapy and or bariatric surgery may be discussed.

\subsubsection{Challenges and Opportunities to the Individual-Based Approach}

One challenge to the individual-based approach is that it is labour intensive requiring input from many healthcare professionals. According to the new clinical practice guidelines a second challenge to this approach is the requirement of increased collaboration between disciplines and the need to establish a Health Team of multidisciplinary professionals in order to deliver the services recommended. Although the province of NL does not have a multidisciplinary approach to the treatment and management of adult obesity, the pediatric hospital in St.John's, NL has established an

Obesity Lifestyle multidisciplinary clinic. The recommended management and treatment options may be associated with increased costs in the short-term due to the resources required (e.g., human, physical, financial). The hope is however, that in the long-term, this approach will be more cost-effective and the health of the individuals will improve as the health effects of comorbidities are treated, managed and potentially minimized. Geography may present another challenge as many rural areas have a high prevalence of obesity and a limited number of health professionals providing continuity of care. In areas where the service density is low it may be difficult to deliver the multidisciplinary 
approach. In the case of the Northern region of this province where there is an increased likelihood of being obese for example the availability and access to a range of health professionals is limited. The strength of these new guidelines and the individual-based approach is that they provide health professionals with a long-awaited management plan for obese patients that are evidence-based. In addition, the establishment of the new primary healthcare renewal satellite practices in certain areas of the province may help to support this approach. These satellite sites offer a new and exciting approach to healthcare delivery in this province. The mix of health professionals working in these sites aim to strengthen the balance of services promoting health, preventing illness and injury, and diagnosing and treat episodic and chronic illness. This advance emphasizes a team-based, interdisciplinary approach providing health services where physicians work closely with other physicians and health care professionals both locally and provincially. This fairly recent development is one that would complement the individual-based approach to obesity treatment and management using the new practice guidelines.

\subsubsection{Evolution of the Problem}

Data suggests that adult obesity rates in NL are increasing and according to the current study a large 'pre-disease population' exists (i.e., $39 \%$ of adults are overweight). Based on the tracking of obesity over the life cycle, this pre-disease population is more likely to gain weight over time. In addition, in children of very young ages obesity is an emerging health problem as $25 \%$ of preschool children were classified either as being

overweight or obese in this province. ${ }^{165}$ Individuals will be exposed to the negative health effects of obesity over a longer portion of their lifetime compared with those adults who 
are now obese. As a result, the adverse health consequences such as adult onset diabetes may appear earlier, for example in obese children.

\subsubsection{Population Health Approach}

Due to the large percentage of individuals classified as obese (i.e., 23\%), and the large percentage classified as overweight (i.e., 39\%), it is important to strengthen the focus of policy and resource allocation decisions on population disease prevention and health promotion. This approach is appropriate not only because of the large number of individuals affected by excessive weight in the population, but because there is a general consensus among researchers that increasing body weight has been largely influenced by environmental change. Changing environments (social, behavioral, cultural, community) have resulted in an imbalance of energy intake and energy expenditure. Findings from the current study found individuals across all levels of BMI reported what behaviors that my considered 'unhealthy'. For example, $55 \%$ of total survey respondents reported being inactive and over $66 \%$ reported infrequent consumption of fruits and vegetables. The obese groups reported these behaviors more often.

A synthesis paper published by the CIHI concluded that obesity was a multifactorial problem. Many factors are associated with and predict obesity including individual lifestyle choices, community structure, societal values and public policy. ${ }^{221}$ The authors of the report suggested that policy makers in collaboration with stakeholders must identify and implement programs and policies that target different levels of environmental influence (e.g., local, community, global) in order to have an effective impact on the population prevalence of obesity. Examples of intervention efforts 
designed to have an impact on these levels of influence include; introducing mandatory physical education classes and healthy food choices in schools, providing infrastructure for bike lanes, safe places to walk, recreation areas and partnering with food manufacturers to ensure food products low in fat, sugar and salt are available at affordable prices.

This holistic environmental approach to obesity prevention has been suggested in various reports. ${ }^{222-224}$ One report published in 2001 provided a strategic plan on how to decrease obesity in the population. This report included a diagram illustrating the different factors influencing the prevalence of obesity by acting on the individual and the population as well as the interactions between the two. There factors can be classified as: International (e.g., globalization of markets, development), National (e.g., urbanization, transport, education), Community (public safety, healthcare), Work/Home (leisure activities, family and home). These factors impact the individual at the most basic level, that of energy expenditure and energy intake. This comprehensive diagram is referred to as the Causal Web of social influences on obesity prevalence and may provide guidance to policy makers in the development of a population public health approach to obesity prevention. $^{222}$

\subsubsection{Population Health Intervention}

To provide an evidence-base for the public health approach, the Canadian Population Health Initiative recently published a review of the literature on overweight and obesity in Canada from a population health perspective focusing on successful population health interventions. ${ }^{221}$ The authors concluded that interventions at the 
population level can be successful if there is an integrated, multi-level coordinated approach to policy by stakeholders and policy actors (e.g., government, health professionals, national organizations, community groups and the public). A range of complementary actions such as healthy school policies, work-site exercise facilities and buy-in by food manufacturers are recommended for individuals, groups, communities, institutions and public policy. The report suggested that for obesity prevention efforts to be both successful and sustainable it is mandatory to have political buy-in or political will as this will ensure the commitment of dedicated resources (e.g., time, money and staff) and aid in the implementation of population-based interventions. As well new policies and programs must be integrated into existing structures and frameworks to ensure longterm sustainability.

In NL in 2001, the government initiated a population health approach to improving the health status of Newfoundlanders and it was conveyed in its Strategic Health Plan. Included in the plan was a focus on obesity prevention. The strategic health plan identified three main goals for NL's health and community services system, as well as objectives and targets within each goal. The directions set out under each of the three goals addresses the challenges NL is facing and aims to work towards the best possible solutions. Goals number one and two are aimed at increasing the health status of the population. Goal number one is to: improve the Health Status of the Population of NL and includes specific objectives to; increase healthy behaviors, improve health outcomes and reduce negative impacts of selected diseases and improve healthy growth and development for children and youth. Goal number two is to improve the capacity of 
communities to support health and well-being. Its objectives include: develop and enhance community partnerships and resources that focus on health and well-being; enhance individual and family participation in community groups and organizations which enable them to make healthy decisions.

Other policy options include sin taxes on energy dense foods, fast foods high in fat or foods considered to have low nutrient value. These taxes have been used in an effort to decrease smoking and were relatively easy to implement. In addition the health effects of smoking on both individuals and those exposed to smoke are well evidenced. The causes of obesity are complex and not well understood. It is felt these types of policies are unlikely to be feasible for obesity prevention.

\subsubsection{Challenges and Opportunities to the Public Health Approach}

Challenges associated with a public health approach include: obtaining buy-in from stakeholders in both the public and private industries and developing a long-term vision for better health that may include altering social structures such as neighborhood design, changing school policies, and targeting individual behaviors especially those around energy intake and expenditure.

There are many opportunities emerging supporting the public health approach to obesity prevention. There is evidence that other collaborative public health interventions may have been successful in educating the public on the risks of these behaviors, changing individual and societal behaviors and decreasing negative health effects. Two public health efforts include interventions directed towards: decreasing smoking behavior and educating the public on HIV/AIDS. These successes demonstrate that population 
health interventions work, but may take time and will require the commitment of many. It is recognized that these health issues differ in many ways from the obesity epidenic being experieneed by most western countries today, however there may be similaritics and lessons to be learned from these initiatives. 


\section{References}

1. OECD data 2005, copyright. Canadian Institute for Health Information. Available at http://secure.cihi.ca/cihiweb/en/media 13nov2007 tab2 ehtml (accessed 22 April 2008)

2. World Health Organization Global Report (2005). Preventing Chronic Diseases: a vital investment. Geneva, World Health Organization

3. National Health Expenditure Database (NHEX) (database online). Health Expenditure by use of Funds, by Source of Finance, by Province/Territory and Canada: Canadian Institute for Health Information. Available at http://secure.cihi.ca/cihiweb/en/media_13nov2007_tab2_e.html. (Accessed 22 April 2008)

4. Health Canada, Economic Burden of Illness 1998 Available at http://www.phacaspc.gc.ca/publicat/ebic-femc98/pdf/ebic 1998.pdf (Accessed on 22 April 2008)

5. Jefferson T. Elementary Economic Evaluation in Health Care. BMJ Publishing Group, BMA House, 1996 London

6. The Public Health Agency of Canada, Available at http://www.phacaspc.gc.ca/publicat/cdic-mcc/27-4/pdf/cdic274-1 e.pdf (Accessed on 28 April 2008)

7. Andersen J. Health Services Utilization: Framework and Review. Health Serv Res 1973;184-99

8. Andersen R, Newman J. Societal and Individual Determinants of Medical Care Utilization in the United States. Milbank Mem Fund Q Health Soc 1973;51(1):95-124

9. Andersen R. Revisiting the behavioral model and access to medical care does it matter? J Health Soc Behav 1995;36 (1):1-10

10. Andersen R, Harada N, Chiu V et al. Application of the Behavioral Model to Health Studies of Asian and Pacific Islander Americans. Asian Am Pac Isl J Health 1995;3(2):129-41

11. Gelberg L, Gallagher T, Andersen R et al. Competing Priorities as a Barrier to Medical Care Among Homeless Adults in Los Angeles Am J Public Health, $1997 ; 87(2): 217-20$ 
12. Andersen R, Davidson $\mathbf{P}$ (eds.) Ethnicity, Aging and Oral Health Outcomes: Findings from the ICS-II USA Research Locations, Adv Dent es1997;11(2):199303

13. Chen M, Andersen R, Barnes D et al. Comparing Oral Health Care Systems 1997 Geneva: World Health Organization

14. Gelberg L, Andersen R, Leake B Applying the Behavioral Model to Vulnerable Populations. Health Serv Res, 2000;34(6):1273-302

15. Andersen R, Bozzette $\mathrm{S}$, Shapiro $\mathrm{M}$ et al. Access of Vulnerable Groups to Antiretroviral Therapy Among Persons in Care for - HIV Disease in the U.S. Health Serv Res 2000;35(2):389-416

16. WHO, Global Strategy on Diet, Physical Activity and Health, (http://www.who.int/dietphysicalactivity/media/en/gsfs chronic disease.pdf)

17. World Health Organization (1998). Obesity. Preventing and managing the global epidemic. International Obesity Task Force. Geneva, World Health Organization

18. Oxford English Dictionary $2^{\text {nd }}$ ed. Oxford: Oxford University Press; 2007

19. Online Medical dictionary. Available from http://www.online-medicaldictionary.org/omd.asp? $\mathrm{q}=$ Obesity

20. Garrow J. Quetelet's index as a measure of fatness. Int J Obes 1985;9:147-53

21. World Health Organization (2000). WHO Technical Report Series no.894. Obesity: Preventing and managing the global epidemic. Geneva: World Health Organization

22. Calle $\mathrm{E}$, Thun $\mathrm{M}$, Petrelli $\mathrm{J}$ et al. Body mass index and mortality in a prospective cohort of U.S. adults. N Engl J Med 1999;341(15):1097-105

23. Fontaine $\mathrm{K}$, Redden $\mathrm{D}$, Wang $\mathrm{C}$ et al. Years of life lost due to obesity. JAMA 2003;289(2):187-93

24. Sturm R. The effects of obesity, smoking and drinking on medical problems and costs. Health Aff 2002;21:245-54

25. Katzmarzyk P. The Canadian obesity epidemic 1985-1998. CMAJ 2002;166(8):1039-40 
26. Health Statistics Division, Health Reports, Statistics Canada, 1994/1995 National Population Health Survey [Ottawa], Statistics Canada

27. Health Statistics Division, Health Reports, Statistics Canada, 2005. Health Indicators: The Canadian Community Health Survey 2005 [Ottawa], Health Statistics Division, Health Reports, Statistics Canada

28. Canadian Institute for Health Information, National Health Expenditure Trends, 1975-2006 (Ottawa: CIHI, 2006)

29. Seidell J, Deerenberg I. Obesity in Europe-prevalence and consequences for the use of medical care. Pharmacoeconomics 1994;5(Suppl.1):38-44

30. Sansone R, Sansone L, Wiederman M. The relationship between obesity and medical utilization among women in a primary care setting. Int J Eat Disord 1998;23:161-67

31. Trakas K, Lawrence $\mathrm{K}$, Shear N. Utilization of health care resources by obese Canadians. CMAJ 1999;160(10):1457-62

32. Reidpath D, Crawford D, Tilgner L et al. Relationship between body mass index and the use of healthcare services in Australia. Obes Res 2002;10:526-31

33. Guayar-Castillon P, Lopez G, Palacios L et al. The relationship of overweight and obesity with subjective health and use of health-care services among Spanish Women. Int J Obes 2002;26:247-52

34. Luchsinger J, Lee $\mathrm{W}$, Carrasquillo $\mathrm{O}$ et al. Body mass index and hospitalization in the elderly. J Am Geriatr Soc 2003;51:1615-20

35. Popoola F, Increase in obesity and health-care use, from the Health Survey for England, Ages 50-69,1998 Obes Surg 2004;14:1258-62

36. Zizza C, Herring A, Stevens J et al. Length of hospital stay among obese individuals. Am J Public Health 2004;94(9):1587-91

37. Centre for Health Information. Obese are three times as likely to need a hip or knee replacement. In: 2005 CJRR Report: Total Hip and total Knee Replacements in Canada Http://secure.cihi.ca/cihiweb/dispPage.jsp?cw page=media 17aug2005 e Accessed on October $18^{\text {th }}, 2005$ 
38. Bertakis K, Azari R. Obesity and the use of health care services. Obes Res 2005;13(2):372-79

39. Levy E, Levy P, Le Pen C et al. The economic cost of obesity: the French situation. Int J Obes Relat Metab Disord 1995;19:788-92

40. Heithoff $\mathrm{K}$, Cuffel B, Kennedy $\mathrm{S}$ et al. The association between body mass index and health care expenditures. Clinical Therapy 1997;19:811-20

41. Quesenbury C Jr, Caan B, Jacobson A. Obesity, health services use, and health care costs among members of a health maintenance organization. Arch Intern Med 1998;158:466-72

42. Thompson D, Brown J, Nichols G, Elmer P, Oster G. Body mass index and future healthcare costs: A retrospective cohort study. Obes Res 2001;9:210-18

43. Finkelstein E, Fiebelkorn I, Wang G. National medical spending attributable to overweight and obesity: how much and who's paying? Health Aff 2003;(Suppl W3):219-26

44. Raebel M, Malone D, Conner D et al. Health services use and health care costs of obese and non obese individuals. Arch Inter Med 2004;164:2135-40

45. Andreyeva T, Sturm R, Ringel J. Moderate and severe obesity have large differences in health care costs. Obes Res 2004;12(12):1936-43

46. Birmingham C, Muller J. Palepu A et al. The cost of obesity in Canada. CMAJ 1999; 164(4):483-88

47. Katzmarzyk P, Janssen I. The economic costs associated with physical inactivity and obesity in Canada: an update. Can J Appl Physiol 2004;29(1):90-115

48. Colditz G. Economic Costs of obesity. Am J Clin Nutr 1992;55(2 Supp 1):503S$507 \mathrm{~S}$

49. Wolf A, Colditz G.The cost of obesity: the US perspective. Pharmacoeconomics 1994;5:34-7

50. Wolf A, Colditz G. Current estimates of the economic costs of obesity in the United States. Obes Res 1998;6:97-106

51. Colditz G. Economic Costs of obesity and inactivity. Med Sci Sports Exerc 1999;31:S663-S667 
52. Siedell J. The impact of obesity on health status: some implications for health care costs. Int J Obes 1996;19(Suppl 6):S13-S16

53. Allison D, Zannolli R, Narayan K. The direct health eare costs of obesity in the United States. Am J Public I/erlth 1909;89:1194-99

54. Hakkinen U. The production of health and the demand for health care in Finland. Soc Sci Med 1991;33:225-37

55. Hodgon T, Meiners M. Cost-of-illness methodology: a guide to current practices and procedures. Milbank Mem Find () 1982:60;429-62

56. Segal L, Carter R, Zimmet P. The cost of obesity. The Australian perspective. Pharmacoeconomics 1994:5(suppl 1):45-52

57. Swinburn B, Ashton T. Gillespie J et al. Health eare costs of obesity in New Zealand. Int J Obes Rela Metab Disord 1997:21:891-96

58. Burnette D, Mui A. Physician utilization by Hispanic elderly persons, National perspective. Med Care 1999;37(4):362-74

59. Epstein A, Cumella E. Capitation payment using predictors of medical utilization to adjust rates. Jealth 'are Financ Rev 1988;10(1):51-69

60. Hulka B, Wheat J. Patterns of utilization, the patient perspective. Med Care $1985 ; 23(5): 438-60$

61. Starrett R, Rogers D, Walters G. Home health care utilization: a causal model. Home Ilealth Care Serv Q 1988;9(4):125-40

62. Ward R. Services for older people: an integrated framework for research. I Health Soc Behav 1977; 18:61-70

63. Finlayson M. DalMonte J. Predicting the use of occupational therapy services among people with multiple sclerosis in Atantic Canada. Can .J Oceap Ther $2002 ; 69(4): 239-48$

64. Parboosing E, Larsen D. Factors influencing frequency and appropriateness of utilization of the emergency room by the elderly. Med ('are 1987;25(12):1139)47 
65. Aday LA, Andersen R. A framework for the study of access to medical care. Hlth Serv Res 1974;9(3):208-20

66. Bradley E, McGraw S, Cury L et al. Expanding the Andersen model: the role of psychosocial factors in long-term care use. Hlth Serv Res 2002;37(5):1221-42

67. McWhinney I. Beyond diagnosis - an approach to the integration of behavioral science and clinical medicine. NEJM 1972;287:384-87

68. Fontaine $\mathrm{K}$, Faith M, Allison D et al. Body weight and health care among women in the general population. Arch Fam Med 1998; 7:381-84

69. Ostbye T, Taylor D, Jung S. A Longitudinal study of the effects of tobacco, smoking and other modifiable risk factors on ill-health in middle-aged and old Americans:Results from the Health and Retirement Study and Asset and Health Dynamics among the Oldest Old Survey. Prev Med 2002;34:334- 45

70. Leon-Munoz L, Garcia E, Ranegas J et al. Relationship of BMI, waist circumference, and weight change with use of health services by older adults. Obes Res 2005;13(8):1398-1404

71. von Lengerke $\mathrm{T}$, Happich $\mathrm{M}$, Reitmeir $\mathrm{P}$ et al. Utilization of out and inpatient health services by obese adults: a population based study in the Augsburg region, Germany. Gesundheitswesen 2005;67(1):S150-S157

72. Meisinger $\mathrm{C}$, Heier $\mathrm{M}$, Lowel $\mathrm{H}$. The relationship between body weight and health care among German women. Obes Res 2004;12(9):1473-80

73. van Dijk L, otters $\mathrm{H}$, Schuit A. Moderately overweight and obese patients in general practice:a population based survey. BMC Fam Pract 2006;7(43)

74. Peytremann-Bridevaux I,Santos-Eggimann B. Healthcare utilization of overweight and obese Europeans aged 50-79 years. J Public Health 2007; 15:377-84

75. Hart C, Hole J, Lawlor D et al. Obesity and use of acute hospital services in participants of the Renfrew/Paisley study. J Public Health 2006;29(1):53-66

76. Counterweight Project Team. Obesity impacts on general practice appointments. Obes Res 2005;13(8):1442-49

77. Chen Y, Jiang Y, Mao Y. Hospital admissions associated with body mass index in Canadian adults. Int Journal of Obesity 2006;31(6):962-7 
78. Payne J, Coy J, Patterson S et al. Is use of hospital services a proxy for morbidity? A small area comparison of the prevalence of arthritis, depression, dyspepsia, obesity and respiratory disease with inpatient admission rates for these disorders in England. J Epidemiolo Community Health 1994;48:74-8

79. Thompson D, Wolf A. The medical-care cost burden of obesity. Obes Rev $2001 ; 2(3): 189-97$

80. Joung I, van der Meer J, Mackenbach J. Marital Status and health care utilization. Int J Epiemiol 1995;24(3):569-75

81. Daviglus M, Liu K, Greenland $\mathrm{P}$ et al. Benefit of a favorable cardiovascular riskfactor profile in middle age with respect to Medicare costs NEJM 1998;339(16):1122-9

82. Simpson L, Owens P, Zodet M. Health care for children and youth in the United States: annual report on patterns of coverage, utilization, quality, and expenditures by income. Ambul Pediatr 2005;5(1):6-44

83. Green C, Pope C. Gender, psychosocial factors and the use of medical services: a longitudinal analysis. Soc Sci Med 1999;48(10):1363-72

84. Finkelstein $\mathrm{M}$. Do factors other than need determine utilization of physicians services in Ontario? CMAJ 2001;165(5):565-70

85. Bertakis K, Azari R, Helms L et al. Gender differences in the utilization of health care services J FAM Pract 2000;49(2) 147-52

86. Kopelman P. Obesity as medical problem. Nature 2000;404:635-43

87. Brodie D, Moscrip V, Hutcheon R. Body Composition Measurement: a review of hydrodensitometry, anthropometry and impedance. Nutrition 1998;14(3):296340

88. Willett W, Dietz W, Colditz G. Guidelines for healthy weight. NEJM 1999;341(6):427-34

89. Ross R, Leger L, Morris D et al. Quantification of adipose tissue by MRI: relationship with anthropometric variables. $J$ Appl Physiol 1992;72(2):787-95

90. Han T, Feskens E, Lean M et al. Waist circumference reduction and cardiovascular benefits during weight loss in women. Int J Obes 1997;21:127-34 
91. Lean M, Han T, Deurenberg P. Predicting body composition by densitometry from simple anthropometric measurements. Am J Clin Nutr 1996;63(1):4-14

92. Zimmet $\mathrm{P}$, Collins $\mathrm{V}$, de Courten $\mathrm{M}$ et al. Is their a relationship between leptin and insulin sensitivity independent of obesity? A population based study in the Indian Ocean of Mauritius. Mauritius NCD Study Group. Int J Obes Relat Metab Disord 1998;22(2):171-77

93. Despres J, Lemieux I, Prud'homme D. Treatment of obesity: need to focus on high risk abdominally obese patients. BMJ 2001;322:716-20

94. Janssen I, Katzmarzyk P, Ross R. Waist circumference and not BMI explains obesity related health risk. Am J Clin Nutr 2004;79(3):379-84

95. Chan D, Watts G, Barrett $P$ et al. Waist circumference, waist-to-hip ratio and body mass index as predictors of adipose tissue compartments in men. QJM 2003;96:441-47

96. Seidell J, Kahn H, Williamson D et al. Report from the Centers for Disease Control and Prevention: Workshop on use of adult anthropometry for public health and primary health care. Am J Clin Nutr 2001;73:123-26

97. Lemieux S, Prud'homme D, Bouchard C et al. A single-threshold value of waist girth identifies normal-weight and overweight subjects with excess visceral adipose tissue. Am J Clin Nutr 1996:64(5):685-93

98. Health Canada. (2003). Canadian guidelines for body weight classification in adults. Retrieved July 21,2004

99. International Diabetes Federation. The IDF consensus worldwide definition of metabolic syndrome. Brussels, Belgium. Available at ww.idf.org/webdata/doc/ Metac_syndrome_def.pdf.

100. Okoson I, Cooper R, Rotimi C et al. Association of waist circumference with risk of hypertension and type 2 diabetes in Nigerians, Jamacians and AfricanAmericans. Diabetes Care 1998;21:1836-42

101. Okoson I, Rotimi C, Forrester T et al. Predictive value of abdominal obesity cutoffs for hypertension in Blacks from West African and Caribbean Islands nations. Int J Obes 2000;24:180-86 
102. Seidell J, Perusse L, Despres J et al. Waist and hip circumferences have independent and opposite effects on cardiovascular disease risk factors: the Quebec Family Study. Am J Clin Nutr 200 lb;74:315-321

103. World Health Organization. Physical Status: The Use and Interpretation of Anthropometry. Technical Report Series 854. Geneva Switzerland: World Health Organization; 1995

104. Bigaard J, Frederiksen K, Tjonneland A et al. Waist and hip circumferences and all-cause mortality: Usefulness of the waist-to-hip ratio? Int J Obes 2004;28:741-47

105. Yusuf S, Hawken S, Ounpuu S et al. Obesity and the risk of myocardial infarction in 27000 participants from 52 countries: a case control study. Lancet 2005;366(9497):1640-49

106. Klipstein-Grobusch $\mathrm{K}$, Georg T, Boeing $\mathrm{H}$. Interviewer variability in anthropometric measurements and estimates of body composition. Int $J$ Epidemiol 1997;26(S1):S174-180

107. Deurenberg. P, Deurenberg-Yap M, Guricci S. Asians are different from Caucasians and from each other in their body mass index/body fat percent relationship. Obes Rev 2002;3:141-146

108. Swinburn BA, Ley SJ, Carmichael HE, Plank LD. Body size and composition in Polynesians. Int J Obes 1999;23(11):1178-83

109. Rush E, Plank L, Coward W. Energy expenditure of young Polynesian and European women in New Zealand and relations to body composition. Am J Clin Nutr.1999:69(1):43-8

110. Flynn M, Gibney M. Obesity and health: why slim? Proc Nutr Soc.1991;50(2):413-32

111. Prentice A, Jebb S. Beyond Body Mass Index. Obe Rev 2001:2(3);141-7

112. Stevens J, Cai J, Jones D. The effect of decision rules on the choice of a body mass index cutoff for obesity: example from African American and white women. Am J Clin Nutr 2002;75:986-92

113. WHO Expert Consultation. Appropriate body-mass-index for Asian populations and its implications for policy and intervention strategies. Lancet 2004;363(9403):157-63 
114. Van Pelt RE, Evans EM, Schechtman KB et al. Waist circumference versus body mass index for prediction of disease in postmenopausal women. Int J Obes $2001 ; 25: 1183-8$

115. Depres JP, Lemieux 1, Prud'homme D. Treatment of obesity: need to focus on high risk abdominally obese patients. $B M J 2001 ; 322: 716-20$

116. Wellens R, Roche A, Khamis $\mathrm{H}$ et al. Relationships between the body mass index and body composition. Obes Res 1996;4(1):35-44

117. Pi-Sunyer FX. Obesity: criteria and classification. Symposium on body weight regulation and obesity: metabolic and clinical aspects $1^{\text {st }}$ plenary session:

obesity. Proc Nutr Soc 2000;59(4):505-09

118. Health Canada. (2003). Canadian guidelines for body weight classification in adults. Accessed on July 21, 2004 www.hc-sc.gc.ca/hpfb-dgpsa/onppbppn/weight_book_tc_e.html

119. Williams Textbook of Endocrinology $10^{\text {th }}$ ed. Philadelphia, Pa:Saunders;2003

120. Wilson P, D’Agostino R, Sullivan L et al. Overweight and obesity as determinants of cardiovascular risk: the Framingham experience. Arch Intern Med 2002;162:1867-72

121. Brown $\mathrm{C}$, Higgins $\mathrm{M}$, Donato $\mathrm{K}$ et al. Body mass index and the prevalence of hypertension and dyslipidemia. Obes Res 2000;8:605-19

122. Burt V, Cutler J, Higgins $\mathbf{M}$ et al. Trends in the prevalence, awareness, treatment and control of hypertension in the adult US population. Hypertension 1995;26:60-9

123. Reeder B, Angel A, Ledoux $\mathrm{M}$ et al. Obesity and its relation to cardiovascular disease risk factors in Canadian adults. Canadian Heart Health Surveys Research Group. CMAJ 1992;146(11):2009-19

124. Abbasi F, Brown B Jr, Lamendola C et al. Relationship between obesity, insulin resistance and coronary heart disease risk. J Am Coll Cardiol 2002; 40(5):93743

125. Bogers R, Bemelmans W, Hoogenveen R et al. Association of overweight with increased risk of coronary heart disease partly independent of blood pressure and cholesterol levels: a meta-analysis of 21 cohort studies including more than 300 , 000 persons. Arch Intern Med 2007;167(16);1720-8 
126. Manson J, Colditz F, Stampfer M et al. A prospective study of obesity and risk of coronary heart disease. NEJM 1990;322:882-89

127. Rexrode K, Hennekens C, Willett W et al. A prospective study of body mass index, weight change and risk for stroke in women. JAMA 1997;277:1539-45

128. Hansson P, Eriksson J, Welin L et al. Smoking and abdominal obesity: risk factors for venous thromboembolism among middle aged men" the study of men born in 1913." Arch Intern Med 1999;159:1886-90

129. Pi-Sunyer F. Medical hazards of obesity. Ann Intern Med 1993;119:655-60

130. Huang Z, Jankinson S, Colditz F et al. Dual effects of weight and weight gain on breast cancer risk. JAMA 1997;278:1407-11

131. Potter J, Slattery M, Bostick R et al. Colon cancer: a review of the epidemiology. Epidemiol Rev 1993;15:499-545

132. Carmichael A. Obesity and prognosis of breast cancer. Obes Rev 2006;7:33340

133. Astrup A, Finer N. Redefining type 2 diabetes: diabesity or obesity dependent diabetes mellitus. Obes Rev 2000;1:57-59

134. Chan J, Rimm E, Colditz G et al. Obesity, fat distribution, and weight gain as risk factors for clinical diabetes in Men. Diabetes Care 1994;17:961-69

135. Colditz G, Willett W, Rotnitzky A et al. Weight gain as a risk factor for clinical diabetes mellitus in women. Ann Intern Med 1995;122:481-86

136. Must A, Spadano J, Coakley E et al. The disease burden associated with overweight and obesity. JAMA 1999;282(16):1523-29

137. Winkleby M, Jautilus D, Frank E et al. Socioeconomic status and health: how education income and occupation contribute to risk factors to cardiovascular disease. Am J Public Health 1992;82(6):816-20

138. Grundy et al. Diagnosis and management of the metabolic syndrome, An American Heart Association/National Heart Lung and Blood Institute Scientific Statement Circulation 2005 accessed December $14^{\text {th }}, 2006$ http://circ.ahajournals.org/cgi/reprint/CIRCULATIONAHA.105.169404v1

139. Ford E, Giles W, Dietz W. Prevalence of the Metabolic Syndrome among US Adults. JAMA 2002;287:356-59 
140. Brien S, Katzmarzyk P. Physical activity and the metabolic syndrome. Appl Physio Nutr Metab 2006;31(1):40-7

141. Pearson-Ceol J. Literature review on the effects of obesity on knee osteoarthritis. Orthop Nurs 2007;26(5):289-92

142. Cicuttini F, Baker J. Spector T. The association of obesity with osteoarthritis of the hand and knee in women: a twin study. J Rheumatol 1996;23:1221-26

143. Enzi G. The socioeconomic consequences of obesity: the effect of obesity on the individual. Pharmacoeconomics 1994;5(S1):S54-7

144. Gortmaker S, Must A, Perrin J. Social and economic consequences of overweight in adolescence and young adulthood. NEJM 1993;329(24):1008-12

145. Lee I-M, Manson J. Body weight and mortality: What is the shape of the curve? Epidemiology 1998: 9(3);227-28

146. Manson J, Stampfer J, Hennekens C et al. Body weight and longevity: a reassessment. JAMA 1987:257-358

147. Manson J, Willett V, Stampfer M et al. Body weight and mortality among women. NEJM 1995;333:677-85

148. Stevens J, Cai J, Pamuk E et al. The effect of age on the association between body-mass index and mortality. NEJM 1998: 338;1-7

149. Sojostrom L. Impacts of body weight, body composition and adipose tissue distribution on morbidity and mortality in Obesity: Theory and therapy, $2^{\text {nd }}$ ed A.J. Stundard and TA Wadden,New York, Raven Press

150. Romero-Corral A, Montori V, Somers V et al. Association of bodyweight and total mortality with cardiovascular events in coronary artery disease: a systematic review of cohort studies. The Lancet 2005;368:666-78

151. Rossner S, Lagerstrand L, Persson $\mathrm{H}$ et al. The sleep apnea syndrome in obesity: risk of sudden death. J Intern Med 1991;230(2):135-141

152. Mulrow C, Chiquette E, Angel L et al. Dieting to reduce body weight for controlling hypertension in adults. Cochrane Database Syst Review 2000; (2):CD000484

153. Goldstein D. Beneficial health effects of modest weight loss. Int J Obesity 1992;16(6):397-415 
154. Ben-Dove I, Grossman E, Stein A et al. Marked weight reduction lowers resting and exercise blood pressure in morbidly obese subjects. Am J Hypertens 2000;13:251-55

155. Dattilo A, Kris-Etherton P. Effects of weight reduction on blood lipids and lipoproteins: a meta-analysis. Am J Clin Nutri 1992;56(2):320-28

156. Sojostrom C, Lissner $\mathrm{L}$, Wedel $\mathrm{H}$ et al. Reduction in incidence in diabetes, hypertension, lipid disturbances after intentional weight loss induced by bariatric surgery: the SOS intervention study. Obes Res 1999;7(5):477-84

157. Wei M, Kampert J, Barlow C et al. Relationship between low cardio respiratory fitness and mortality in normal-weight, overweight, and obese men. JAMA 1999;282(16): 1547-53

158. Sui X, LaMonte M, Laditka J et al. Cardiorespiratory Fitness and adiposity as mortality predictors in older adults. JAMA 2007;298(21):2507-16

159. Janssen I, Katmarzyk P, Ross R. Body mass index is inversely related to mortality in older people after adjustment for waist circumference. J Am Geriatr Soc 2005;53(12):2112-28

160. Baskin M, Ard J, Franklin F et al. Prevalence of obesity in the United States. Obes Rev 2005;6:5-7

161. Flegal K, Toriano R. Changes in the distribution of the body mass index of adults and children in the US population. Int J Obes 2000;24:807-18

162. Belanger-Ducharme F, Tremblay A. Prevalence of obesity in Canada. Obes Rev 2005;6:183-86

163. Tremblay M, Katzmarzyk P, Willms J. Temporal trends in overweight and obesity in Canada 1981-1996, Int J Obes Relat Metab Disord 2002;26(4):538-43

164. Willms J, Tremblay M, Katzmarzyk P. Geographic and demographic variation in the prevalence of overweight Canadian children. Obes Res 2003;11(5):668-73

165. Canning P, Courage M, Frizell L. Prevalence of overweight and obesity in a provincial population of Canadian preschool children. CMAJ 2004;171(3):240-2

166. Twells L, Day S, Newhook LA. Does breastfeeding protect preschool children from obesity? Atlantic Networks for Prevention Research Conference, St. John's, NL (Abstract) 2007 
167. Lissner L. Causes, diagnosis and risks of obesity. Pharmacoeconomics 1994;5(Suppl 1):8-17

168. Pi-Sunyer F. Obesity: criteria and classification. Symposium on body weight regulation and obesity: metabolic and clinical aspects $1^{\text {st }}$ plenary session obesity. Proc Nutr Soc 2000;59:505-09

169. Canadian Population Health Initiative (2004). Improving the Health of Canadians. Canadian Institute for Health Information. Ottawa

170. Prentice A, Jebb S. Obesity in Britain: gluttony or sloth? BMJ 1995;311(7002):437-9

171. Schulze L, Bennett $P$, Ravussin E et al. Effects of tradition and western environments on prevalence of Type II diabetes in Pima Indians in Mexico and the USA. Diabetes Care 2006:29(8):1866-71

172. O'Dea K, White N, Sinclair A. An Investigation of nutrition -related risk factors in an isolated Aboriginal community in northern Australia - advantages of a traditionally oriented lifestyle. Med J Aust 1988;148:177-180

173. Harris S, Gittelsohn J, Hanley A et al. The prevalence of NIDDM and associated risk factors in native Canadians. Diabetes Care 1997;20:185-7

174. Young TK., Reading J, Elias B and O' Neil JD. 2000 Type 2 diabetes mellitus in Canada's First Nations: status of an epidemic in progress. CMAJ 163;561-66

175. Delisle H. Rovard M, Ekoe J. Prevalence estimates of diabetes and other cardiovascular risk factors in the two largest Algonquin communities in Quebec. Diabetes Care 1995;18:1255-9

176. Hill J, Catenacci V, Wyatt H. Obesity. Etiology in Modern Nutrition in Health and Disease $10^{\text {th }}$ ed. Lippincott Williams \& Wilkins:2006

177. Kromhout D. Changes in energy and macronutrients in 871 middle-ages men during 10 years of follow-up (the Zutphen study). Am J Clin Nutr 1983:37:287

178. Nova Scotia Heart Health Program. (1993). Report of the Nova Scotia Nutrition Survey. Nova Scotia: Nova Scotia Department of Health

179. University of Saskatchewan. (2001). Saskatchewan Nutrition Survey. Report of a Survey in the province of Saskatchewan, 1993-1994. Saskatoon: University of Saskatchewan 
180. Taylor J, Van Til L, MacLellan D. (2002). Prince Edward Island Nutrition Survey. Charlottetown:University of Prince Edward Island and Prince Edward Island Health and Social Services

181. Gray-Donal K, Jacobs-Starkey L, Johnson-Down L. Food habits of Canadians: Reduction in fat intake over a generation. Ca J Public Health 2000;91:381-385

182. Stephen A, Sieber G. Trends in individual consumption of dietary fat in the United States, 1920-1984. Am J Clin Nutr 1994;,52:457-469

183. Statistics Canada(2003). Food Statistics,2002. Volume 2, number 1. Ottawa; Statistics Canada. www.statcan.ca/english/freepub/21-020-XIE/21-020XIE02001.pdf

184. Roebothan B Nutrition Newfoundland and Labrador: The Report of a Survey of Residents of Newfoundland and Labrador, 1996. St. John's, Newfoundland: Department of Health and Community Services, Province of Newfoundland and Labrador 2003

185. Fogelholm M, Mannisto S, Vartianen E et al. Determinants of energy balance and overweight in Finland 1982 and 1992. Int J Obes Relat Metab Disord 1996;20:1097-104

186. US Department of Health and Human Services. Physical Activity and Health: A Report of the Surgeons General. Atlanta, GA: US Department of Health and Human Resources, 1996

187. Viswanaath $\mathrm{K}$, Bond $\mathrm{K}$. Social determinants and nutrition: reflections on the role of communication. $J$ Nutr Edu Behav 2007;39(Suppl 2): S20-4

188. Sobal J, Stunkard A. Socioeconomic status and obesity: a review of the literature. Psychol Bull 1989;105(2):260-75

189. Ball K, Crawford D. Socioeconomic status and weight change in adults: a review. Soc Sci Med 2004;60(9):1987-2010

190. Bell, C, Walley A, Froguel P. The genetics of human obesity. Nat Rev Genet 2005;6:221-34

191. Zhang Y, Proenca R, Maffei R et al. Positional cloning of the mouse obese gene and its human homologue. Nature 1994;372(6505):425-32 
192. Barness L, Opitz J, Gilbert-Barness E. Obesity: Genetic, Molecular, and Environmental Aspects. Am J of Medical Genetics Part A2007;143A:3016-34

193. Allison D, Mentore J, Heon M et al. Antipsychotic - induced weight gain: a comprehensive synthesis. Am J Psychiatry 1999;156:1686-96

194. Bouchard C. Etiology of obesity: genetics factors. Arch Latinoam Nutr 1992;42(3 Suppl):127S-130S

195. Rankinen T, Zuberi A, Chagnon Y et al. The human obesity gene map: the 2005 update. Obesity 2006;14(4):529-644

196. Stunkard A, Foch T, Hrubec $\mathrm{Z}$ et al. A twin study of human obesity. JAMA 1986;256(1):51-4

197. Meyer J, Stunkard A. Twin studies of human obesity. In Ed: Bouchard C. The genetics of obesity. Boca Raton: CRC Press. 1994:63-78

198. Bouchard C, Tremblay A, Despres J et al. The response to long-term overfeeding in identical twins. NEJM 1990;322(21):1477-82

199. Jacobson P, Torgerson J, Sjostrom L et al. Spouse resemblance in body mass index: effects on adult obesity prevalence in the offspring generation. $A m J$ Epidemiol 2007;1(165):101-8

200. Statistics Canada: Canadian Community Health Survey 2001 located at www.statcan.ca/english/sdds/instrument/3226_Q1_V1_E.pdf

201. Statistics Canada: Canadian Community Health Survey 2001 located at http://www.statcan.ca/english/concepts/hs/index.htm

202. Statistics Canada: Canadian Community Health Survey 2001 located at http://www.statcan.ca/english/freepub/82-221-XIE/free.htm

203. Canadian Institute for Health Information: Resource Intensity Weights http://www.cihi.ca/cihiweb/dispPage.jsp?cw page=casemix riw e

204. Financial Performance Indicators 1999-2000 to 2001-2002 Canadian Institute for Health Information. Canadian MIS Database Hospital

205. Boreham G, Robson P, Gallagher A et al. Tracking of physical activity, fitness, body composition and diet from adolescence to young adulthood. The Young Hearts Project, Northern Ireland. Int J Behav Nutr Phys Act 2004;1(1):14 
206. Kyaavik E, Tell G, Klepp K. Predictors and tracking of body mass index from adolescence into adulthood: follow up of 18 to 20 years in the Oslo Youth Study. Arch Pediatr Adolesc Med 2003;157(12):1212-18

207. Fuentes R, Notkola I, Shemeikka S. Tracking of body mass index during childhood: a 15 year prospective population-based family study in eastern Finland. Int J Obes Relat Metab Disord 2003; 27(6):716-21

208. Erikson J, Forsen T, Tuomilehto J et al. Size at birth, childhood growth and obesity in adult life. Int J Obes Relat Metab Disord 2001;25(5):735-40

209. Shields M, Tjepkema M. Trends in adult obesity in Health Reports (Statistics Canada, Catalogue 82-003) 2006;17(3):9-25

210. Kirkwood Betty. Essentials of Medical Statistics. Oxford. Blackwell Science Ltd.;1988

211. Munroe, B Statistical Methods for Health Care Research $3^{\text {rd }}$ ed. Lippincott

212. Harrison G, Feehan J, Edwards A et al. Cigarette smoking and the cost of hospital and physician care. Can Public Policy 2003;29(1):1-20

213. Ramsey F, Schafer D. The Statistical Sleuth: A Course in Methods of Data Analysis. $2^{\text {nd }}$ ed. New York. Duxbury 2002

214. Hurley J, Hutchison B, Buckley G et al. Needs-based Funding for Home Care and Community Support Services in Ontario: A New Approach Based on Linked Survey and Administrative Data. CHEPA Working Paper Series, paper 03-01 2003

215. MacKinnon D, Krull J, Lockwood C. Equivalence of the Mediation, Confounding and Suppression Effect. Prevention Science 2000;1(4):173-81

216. Beaglehole R, Bonita R, Kjellström T. Basic Epidemiology. $1^{\text {st }}$ ed. Geneva: World Health Organization; 1993

217. Borg S, Persson U, Odegaard K et al. Obesity, survival and hospital cost findings from a screening project in Sweden. Value Health 2005;8(5):562-71

218. Arterburn D, Maciejewski M, Tsevat J. Impact of morbid obesity on medical expenditures in adults. Int $J$ of Obes 2005;29:334-39 
219. Catford J. Chronic disease: preventing the world's next tidal wave the challenge for Canada 2007. Ilealth Promot Im 2007;22:1-4

220. Lau D, Douketis J, Morrison K et al. 2006 Canadian clinical guidelines on the management and prevention of obesity in adults and children [summary]. (M.1.J $2007 ; 176(8): S 1-13$

221. Raine K. Overweight and obesity in Canada. A population health perspective. 2004. Canadian Institute for Health Information, Canadian Population Health Initiative [Ottawa]

222. Kumanyika S. Minisymposium on Obesity: Overview and some strategic considerations Annu Rev Public Ilealth 2001;22:293-308

223. Hirsh J. Magic bullet for obesity. Clinical Review BMJ 1998;317:1136-8

224. Butchko H, Petersen B. The Obesity Epidemic: Stakeholder Initiatives and Cooperation. Nutr Todav 2004:39(6):235-44 


\section{APPENDIX A Andersen's Behavioral Model of Health Services Use}




\section{ENVIRONMENT POPULATION CHARACTERISTICS

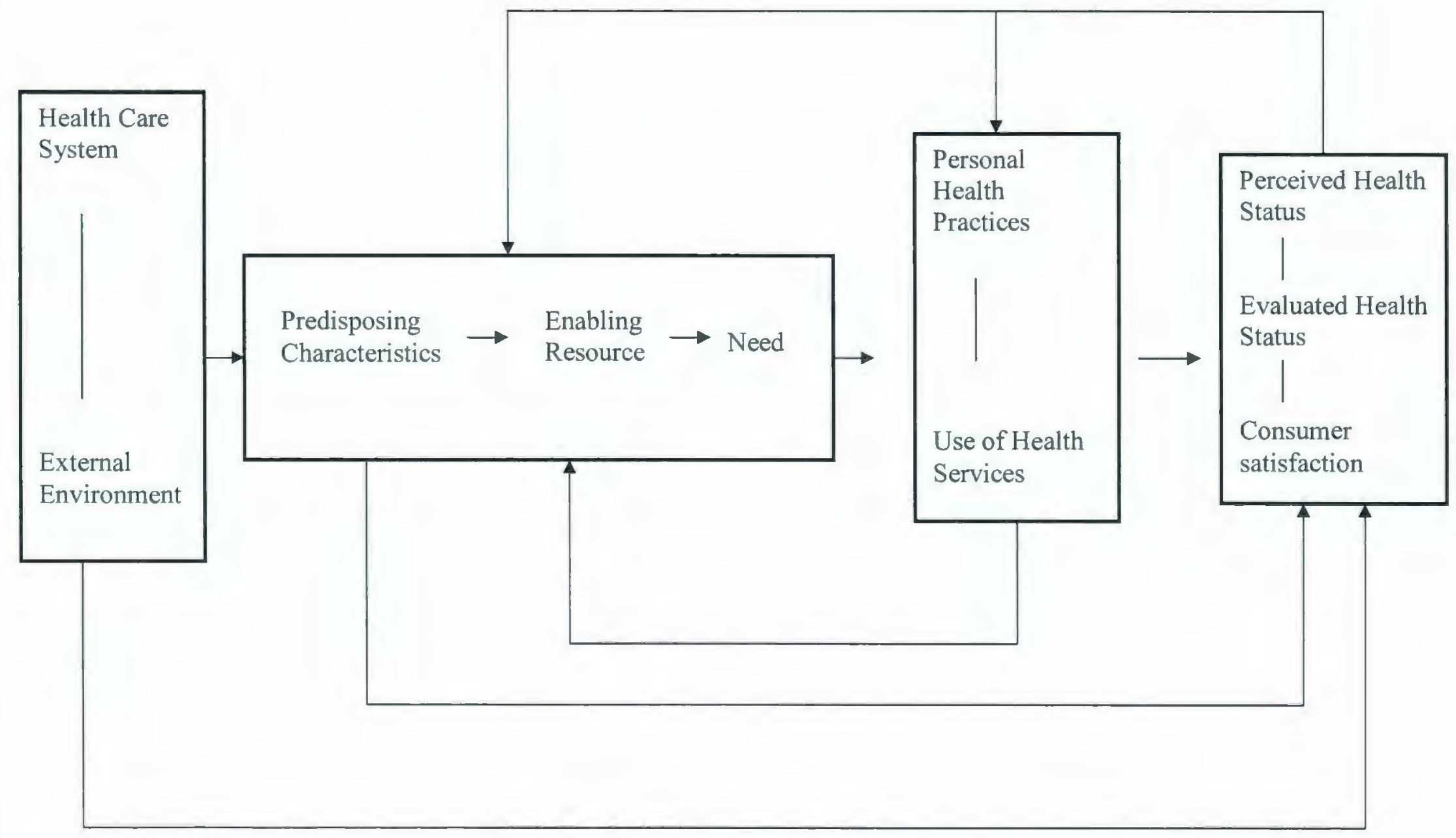




\section{APPENDIX B \\ Detailed Linkage Process}




\section{Detailed Linkage Process}

The linkage of the database took place in four steps and was conducted using SPSS for Windows version 11.5.0. Step I involved linkage of the "share file" to the "link file" and extraction of the health insurance numbers from the "link file." In step two, the health survey study sample was linked to the physician claims database. In step three the health survey study sample was linked to the hospital separation database and in step four the health survey study sample was linked to the Provincial Mortality Surveillance System.

\section{Step I Linkage of share file to link file}

The household ID and person ID variables in the 2000/2001CCHS "share file" and 2000/20001 CCHS “link file" were concatenated to form a new composite ID variable. The "share file" was linked to the "link file" so that the date-of-birth variable in the "share file" could be added to the "link file." The "link file" (containing MCP number, name, date-of-birth) was linked to the MCP "master file" via the MCP number in order to determine the validity of the MCP numbers in the "link file." Out of a potential 3734 survey respondents, 2883 individual records were linked ( $82 \%$ of the link file and $76 \%$ of the share file). Records with missing or invalid MCP numbers could not be linked. For the 614 individual records in the "link file," which could not be linked via MCP numbers, a second link was conducted to obtain valid MCP numbers for as many as possible of the remaining survey participants. A composite variable was created in the "link file" using the first 4 characters of the first name, the first 4 characters of the last name and the dateof-birth. This was done in both the CCHS "link file" and the MCP "master file." This variable was used to link the remaining records in the "link file" to the MCP "master 
file." A total of 382 additional records were linked using this procedure. As a result the new "link file" contained the original valid MCP numbers provided (2833), plus the additional 382 valid MCP numbers obtained form the MCP "master file" to make up a total "linkable" sample of 3265 , bringing the proportion of records with valid MCP numbers to $93 \%$ of the "link file" and $87 \%$ of the "share file." The final step included linkage of the new "NLCHI link file" with its corresponding valid MCP numbers to the CCHS study sample $(n=2345)$ to determine how many of the CCHS study sample $(\mathrm{n}=2345)$ had valid MCP numbers. When this link was completed $2177(92.8 \%)$ of the CCHS study sample had valid MCP numbers and were linked to the health administration databases.

\section{Step II: CCHS Linkage to the Medical Care Plan database}

Objective health services use for physicians was ascertained through linkage of the CCHS to the MCP database via a unique identifier (Medical Care Plan number). A file with the MCP health insurance number and the survey respondent ID was linked to the MCP database to obtain physician utilization for the study sample. Data were obtained on physician visits, fee code, physician fee claimed, and provider's specialty for a five year period (1998-2002).

\section{Step III: CCHS 1.1 Linkage to Clinical Database Management System}

Objective health services hospital use was ascertained through the linkage of the CCHS "link file" to the MCP number in the CDMS. Survey respondents with a valid MCP number were linked to the CDMS. Utilization of hospital services data were collected through the number of hospital separations, information on main diagnoses, type of 
procedures, length-of-stay, resource intensity weights and provider information for those CCHS respondents who had either an admission to hospital or a visit to surgical day care.

\section{Step 4: Linkage Mortality Surveillance System}

Using a valid MCP number, 2200 CCHS respondents were linked to the Mortality

Surveillance System, to detcrmine mortality and cause of death. 


\title{
APPENDIX C
}

\section{Table 1a: Select Measures of Health Behavior from the 2000/2001 CCHS}

\author{
Table 1b: Select Measures of \\ Population Characteristics from the \\ 2000/2001 CCHS
}




\section{Table 1a: Select Variables Measuring Health Behavior (i.e., Health Services Utilization) from the $2000 / 2001$ CCHS}

\begin{tabular}{|c|c|}
\hline \multicolumn{2}{|r|}{ Health Services Utilization variables } \\
\hline $\begin{array}{l}\text { Overnight patient } \\
\text { (HCUA 01) }\end{array}$ & $\begin{array}{l}\text { In the past } 12 \text { months, have you been a patient overnight in a hospital, } \\
\text { nursing or convalescent home? } \\
1=y e s, 2=\text { no }\end{array}$ \\
\hline $\begin{array}{l}\text { Number of nights as a } \\
\text { patient } \\
\text { (HCUAGO1A) }\end{array}$ & $\begin{array}{l}\text { Number of nights respondent was in hospital as a patient in the } 12 \text { month } \\
\text { period prior to the survey interview? }\end{array}$ \\
\hline $\begin{array}{l}\text { Family doctor } \\
\text { (HCUAGO2A) }\end{array}$ & $\begin{array}{l}\text { Number of consultations with family doctor in the } 12 \text { months period prior to } \\
\text { the survey interview }\end{array}$ \\
\hline $\begin{array}{l}\text { Other medical doctor } \\
\text { (HCIAGMDC) }\end{array}$ & $\begin{array}{l}\text { Number of consultations with another medical doctor in the } 12 \text { months prior } \\
\text { to the survey interview? }\end{array}$ \\
\hline $\begin{array}{l}\text { Any health professional } \\
\text { (HCUAFHPC) }\end{array}$ & $\begin{array}{l}\text { Have you had a consultation with any health professional in the } 12 \text { month } \\
\text { period prior to the interview? }\end{array}$ \\
\hline $\begin{array}{l}\text { Mental health professional } \\
\text { (CMHA_01K) }\end{array}$ & Have you consulted a mental health professional in the past 12 months? \\
\hline
\end{tabular}




\section{Table 1b: Select Variables Measuring Population Characteristics from the 2000/2001 CCHS}

\begin{tabular}{|c|c|}
\hline Variable & Population Health Characteristics \\
\hline \multicolumn{2}{|r|}{ Predisposing Variables } \\
\hline $\begin{array}{l}\text { Age } \\
\text { (DHHA AGE) }\end{array}$ & Age \\
\hline Sex & $0=$ Male, $1=$ Female \\
\hline $\begin{array}{l}\text { Marital Status } \\
\text { (DHHA MS) }\end{array}$ & $1=$ married, 2 = common-law, $3=$ widowed, $4=$ separated, $5=$ divorced, $6=$ single \\
\hline $\begin{array}{l}\text { Education level (D) } \\
\text { (EDUADHO4) }\end{array}$ & $\begin{array}{l}\mathrm{l}=<\text { secondary, } 2=\text { secondary, } 3=\text { other post secondary graduate, } 4=\text { post } \\
\text { secondary graduate }\end{array}$ \\
\hline Body mass index (D) & $\begin{array}{l}\text { Weight in kilograms/height in metres squared } \\
1=\text { normal } \mathrm{BMI} \geq 18.5 \text { and }<25,2=\text { overweight } \mathrm{BMI} \geq 25 \text { and }<30 \\
3=\text { obese } \mathrm{BMI} \geq 30\end{array}$ \\
\hline \multicolumn{2}{|r|}{ Enabling Variables } \\
\hline $\begin{array}{l}\text { Health Services } \\
\text { (TWDA 5) }\end{array}$ & $\begin{array}{l}\text { Do you have a regular medical doctor? } \\
1=\text { yes, } 2=\text { no }\end{array}$ \\
\hline $\begin{array}{l}\text { Income (D) } \\
\text { (INCADIA5) }\end{array}$ & $1=$ lowest, $2=$ lower middle, 3 =middle, $4=$ upper middle, $5=$ highest \\
\hline $\begin{array}{l}\text { Rural and Urban } \\
\text { (GEOADUR2) }\end{array}$ & $1=$ urban, $2=$ rural \\
\hline $\begin{array}{l}\text { Health region } \\
\text { (GEOA_PRV) }\end{array}$ & $\begin{array}{l}1001=\text { St. John's, 1002=Eastern, 1003=Central, 1004=Western, 1005=Grenfell, } \\
1006=\text { Labrador }\end{array}$ \\
\hline \multicolumn{2}{|r|}{ Need Variables } \\
\hline $\begin{array}{l}\text { Chronic condition } \\
\text { (CCCAF1) }\end{array}$ & $\begin{array}{l}\text { Do you have a chronic condition? } \\
1=\text { yes, } 2=\text { no }\end{array}$ \\
\hline $\begin{array}{l}\text { Number of chronic conditions (D) } \\
\text { (CCCADTOT) }\end{array}$ & $\begin{array}{l}\text { We are interested in long-term conditions that have lasted or are expected to } \\
\text { last } 6 \text { months or more and have been diagnosed by a health professional. } \\
\text { Do you have? } \\
\text { food allergies, any other allergies, asthma, Fibromyalgia, arthritis or } \\
\text { rheumatism, back problems excluding arthritis and Fibromyalgia, high blood } \\
\text { pressure, migraine headaches, chronic bronchitis or emphysema or chronic } \\
\text { obstructive pulmonary disease, diabetes, epilepsy, heart disease, cancer, } \\
\text { stomach or intestinal ulcers, effects of a stroke, urinary incontinence, a bowel } \\
\text { disease (Crohn's or colitis), Alzheimers's disease or other dementia, cataracts, } \\
\text { glaucoma, thyroid condition, any other long-term condition that has been } \\
\text { diagnosed by a health professional } \\
\text { For arthritis or rheumatism respondents were asked if they suffered from } \\
\text { rheumatoid arthritis, osteoarthritis or other arthritic problems? } \\
\text { For heart disease, respondents were also asked if they had suffered a heart }\end{array}$ \\
\hline
\end{tabular}




\begin{tabular}{|c|c|}
\hline & $\begin{array}{l}\text { attack, angina or congestive heart failure } \\
\text { For cancer, respondents were asked about specific cancers (breast, prostate, } \\
\text { colorectal, skin cancer (melanoma and non melanoma) and other }\end{array}$ \\
\hline & Need Variables - Health Status \\
\hline $\begin{array}{l}\text { Physical Activity Index (D) } \\
\text { (PACDPAI) }\end{array}$ & $\begin{array}{l}\text { Derived physical activity index (based on cstimated energy expenditure) } \\
1 \text { Active, } 2 \text { moderately active, } 3 \text { - inactive }\end{array}$ \\
\hline $\begin{array}{l}\text { Fruit and vegetable } \\
\text { consumption(D) } \\
\text { (FVCAGTOT) }\end{array}$ & $\begin{array}{l}\text { Daily consumption of total fruits and vegelables } \\
1-5 \text { servings per day, } 2 \rightarrow 6 \text { and }<10,3=10 \text { servings per day }\end{array}$ \\
\hline $\begin{array}{l}\text { Alcohol (D) } \\
(A I . C \text { AI)YTP) }\end{array}$ & $\begin{array}{l}\text { Type ol drinker } \\
1 \text { regular, } 2 \text { occasional, } 3=\text { never }\end{array}$ \\
\hline $\begin{array}{l}\text { Smoking } \\
\text { (SMKA 2()2) }\end{array}$ & $\begin{array}{l}\text { Type of smoker } \\
1 \text { daily, } 2 \text { occasional, } 3 \text { not at all }\end{array}$ \\
\hline
\end{tabular}




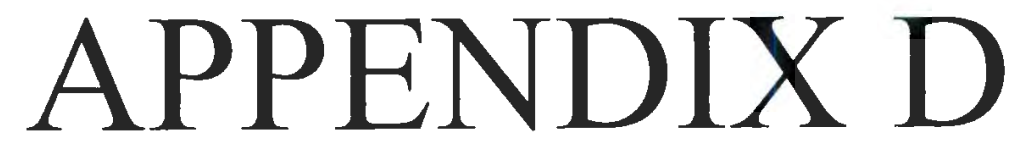

Does Body Mass Index Change over
time? 


\section{Does Body Mass Index Change Over Time?}

The current study identifies obese, overweight and normal weight individuals at a particular point in time and tracks their HSU over a two and a half year period pre and post survey data collection. The possibility that subjects found to be obese in this CCHS, but were not obese pre and or post the survey must be considered.

To further investigate whether BMI/obesity status remains constant over a fiveyear period, the longitudinal portion of the NL sample of the National Population Health Survey (NPHS) Cycles 1 (94/95) and 5 (02/03) were analyzed. The NPHS was first administered to the Canadian population in 1994/95 and has since been repeated every two years. This survey collects information on health status, health behaviors and health services utilization at the provincial level, with both longitudinal and cross-sectional components. The total Canadian sample size was 17,625 in 1994/95. The sample population size for the NL component over this time period was 806. Calculated BMI values were available for 429 individuals between 18 and 64 years of age who were part of the longitudinal component. This information was used to determine the percentage of people classified as obese in 1994/1995 who remained classified as obese in 2002/2003.

Table 1 presents a cross-tabulation of individuals in 1994/1995 and in 2002/03 with their corresponding weight classification.

As the table demonstrates, in 1994/1995, seventy-five adult respondents were classified as obese. In 2002/2003, fifty eight (77\%) of these same respondents were classified as obese. Fourteen, two and one respondent(s) had moved into the overweight, normal weight and underweight categories respectively. In 1994/1995, one hundred and 
seventy-eight adults were classified as normal weight and in 2002/2003, one hundred and ten $(62 \%)$ of these were classified as normal weight. Eight were classified as obese, fiftyseven as overweight and three respondents were classified as underweight. These findings suggest that between 1994/95 and 2002/03, 80\% obese individuals remained obese, $20 \%$ overweight individuals had become obese, $33 \%$ normal weight individuals had become overweight. Less than $10 \%$ overweight individuals had moved into the normal range.

Table 1 NPHS Cross-tabulation of BMI Cycle 1 (1994) and Cycle 5 (2003)

BMI Class - Cycle 5 (2002-2003)

\begin{tabular}{|c|c|c|c|c|c|}
\hline $\begin{array}{l}\text { BMI Class } \\
\text { Cycle } 1 \\
(1994 / 1995)\end{array}$ & $\begin{array}{c}\text { Underweight } \\
(\%)\end{array}$ & $\begin{array}{c}\text { Normal weight } \\
(\%)\end{array}$ & $\begin{array}{c}\text { Overweight } \\
(\%)\end{array}$ & $\begin{array}{c}\text { Obese } \\
(\%)\end{array}$ & Total \\
\hline Underweight & $1(16.7)$ & $5(83)$ & 0 & 0 & 6 \\
\hline Normal & $3(1.7)$ & $110(61.8)$ & $57(32)$ & $8(4.5)$ & 178 \\
\hline Overweight & 0 & $15(8.8)$ & $120(71)$ & $35(21)$ & 170 \\
\hline Obese & $1(1.3)$ & $2(2.7)$ & $14(18.7)$ & $58(77.3)$ & 75 \\
\hline Total & 5 & 132 & 191 & 101 & 429 \\
\hline
\end{tabular}

These analyses are supported by a national study recently published by Statistics Canada in 2005.Using the longitudinal component of the National Population Health Survey for the entire country, researchers found between 1993/1994 and 2002/2003: 25\% of overweight individuals had become obese; $33 \%$ of normal weight individuals had become overweight and $10 \%$ of overweight individuals had moved into the normal range. This analysis reported that men were more likely to go from normal weight to overweight and women were more likely to go from overweight to obese. These specific Canadian 
findings support the use of obesity measured as a cross sectional parameter, as a valid and reliable measure of weight class over time in this population. 
APPENDIX E 
Table 1: Distribution of Physicians in NL 1999/2000

\begin{tabular}{|c|c|c|c|c|c|c|c|}
\hline & \multicolumn{2}{|c|}{ GP } & \multicolumn{2}{|c|}{ Specialist } & \multirow{2}{*}{\begin{tabular}{|l|} 
Total \\
FFS
\end{tabular}} & \multirow{2}{*}{$\begin{array}{c}\text { Total } \\
\text { physicians }\end{array}$} & \multirow{2}{*}{$\begin{array}{c}\text { FFS } \\
\%\end{array}$} \\
\hline & FFS & Salary & FFS & Salary & & & \\
\hline \multicolumn{8}{|l|}{ Health Board } \\
\hline \multicolumn{8}{|l|}{ Eastern } \\
\hline St. Johns & 142 & 13 & 173 & 48 & 315 & 376 & $84 \%$ \\
\hline Avalon & 13 & 5 & 0 & 0 & 13 & 18 & $72 \%$ \\
\hline Trinity/conception & 27 & 2 & 5 & 7 & 32 & 41 & $78 \%$ \\
\hline Burin & 13 & 13 & 3 & 9 & 16 & 38 & $42 \%$ \\
\hline Clarenville & 18 & 6 & 2 & 15 & 20 & 41 & $49 \%$ \\
\hline total & 213 & 39 & 183 & 79 & 396 & 514 & $77 \%$ \\
\hline \multicolumn{8}{|l|}{ Central } \\
\hline Gander and District & 16 & 18 & 14 & 18 & 30 & 66 & $45 \%$ \\
\hline Central & 22 & 12 & 17 & 5 & 39 & 56 & $70 \%$ \\
\hline Port Saunders & 1 & 2 & 0 & 0 & 1 & 3 & $33 \%$ \\
\hline Baie Verte & 5 & 0 & 0 & 0 & 5 & 5 & $100 \%$ \\
\hline Green Bay & 4 & 2 & 0 & 0 & 4 & 6 & $67 \%$ \\
\hline Notre Dame Bay & 1 & 5 & 0 & 0 & 1 & 6 & $17 \%$ \\
\hline Buchans & 0 & 1 & 0 & 0 & 0 & 1 & $0 \%$ \\
\hline total & 49 & 40 & 31 & 23 & 80 & 143 & $56 \%$ \\
\hline \multicolumn{8}{|l|}{ Western } \\
\hline Western & 29 & 13 & 27 & 21 & 56 & 90 & $62 \%$ \\
\hline Stephenville & 8 & 6 & 3 & 7 & 11 & 24 & $46 \%$ \\
\hline Port Aux Basques & 3 & 6 & 0 & 0 & 3 & 9 & $33 \%$ \\
\hline total & 40 & 25 & 30 & 28 & 70 & 123 & $57 \%$ \\
\hline \multicolumn{8}{|l|}{ Grenfell/Labrador } \\
\hline St. Anthony/Goose Bay & 5 & 27 & 0 & 22 & 5 & 54 & $9 \%$ \\
\hline Labrador CityMabush & 6 & 0 & 4 & 3 & 10 & 13 & $77 \%$ \\
\hline \multirow[t]{2}{*}{ total } & 11 & 27 & 4 & 25 & 15 & 67 & $22 \%$ \\
\hline & & & & & 0 & 0 & \\
\hline Total & 313 & 131 & 248 & 155 & 561 & 847 & $66 \%$ \\
\hline
\end{tabular}

Source: Annual Report 1999/2000 Medical Care: Plan Table 5 
Table 2:Analysis of \# of Visits to a FFS Physician by Fee-Code $(n=2177)$

\begin{tabular}{|c|c|c|c|c|c|c|}
\hline \multirow[t]{2}{*}{ Fee-code } & \multicolumn{2}{|c|}{ Total } & \multicolumn{2}{|c|}{ GP visits } & \multicolumn{2}{|c|}{ Specialist visits } \\
\hline & $\mathbf{n}$ & $\%$ & $\mathbf{n}$ & $\%$ & $\mathbf{n}$ & $\%$ \\
\hline $\begin{array}{c}\text { Office } \\
\text { consultation }\end{array}$ & 39116 & 56 & 34477 & 87 & 4639 & 15 \\
\hline $\begin{array}{c}\text { Home } \\
\text { consultation }\end{array}$ & 553 & 1 & 546 & 1 & 7 & 0 \\
\hline $\begin{array}{c}\text { In-patient } \\
\text { consultation }\end{array}$ & 1222 & 2 & 250 & 1 & 972 & 3 \\
\hline $\begin{array}{l}\text { Out-patient } \\
\text { and } \\
\text { emergency }\end{array}$ & 4881 & 7 & 1060 & 3 & 3821 & 12 \\
\hline $\begin{array}{l}\text { Diagnostic } \\
\text { and } \\
\text { therapeutic } \\
\text { procedures }\end{array}$ & 4765 & 7 & 2394 & 6 & 2371 & 8 \\
\hline $\begin{array}{l}\text { In-hospital } \\
\text { diagnostic } \\
\text { procedures }\end{array}$ & 2674 & 4 & 1 & 0 & 2673 & 9 \\
\hline Radiology & 13198 & 19 & 66 & 0 & 13132 & 43 \\
\hline $\begin{array}{c}\text { Surgical } \\
\text { procedures }\end{array}$ & 3703 & 5 & 614 & 2 & 3089 & 10 \\
\hline Total & 70112 & 100 & 39408 & 100 & 30704 & 99 \\
\hline
\end{tabular}


Table 3a: Urban Region

\begin{tabular}{|c|c|c|c|c|c|c|c|c|}
\hline & \multicolumn{2}{|c|}{ Morbidly Obese* } & \multicolumn{2}{|c|}{ Obese* } & \multicolumn{2}{|c|}{ Overweight* } & \multicolumn{2}{|c|}{ Normal* } \\
\hline & Self report & Actual & Self report & Actual & Self report & Actual & Self report & Actual \\
\hline & \multicolumn{2}{|c|}{$n=81$} & \multicolumn{2}{|c|}{$n=197$} & \multicolumn{2}{|c|}{$n=463$} & \multicolumn{2}{|c|}{$n=505$} \\
\hline & $\mathrm{X}(\mathrm{SD})$ & $\mathbf{X}(\mathrm{SD})$ & $\mathrm{X}(\mathrm{SD})$ & $\mathbf{X}(\mathrm{SD})$ & $\mathrm{X}(\mathrm{SD})$ & $X(S D$ & $\mathrm{X}(\mathrm{SD})$ & $X(S D)$ \\
\hline \multirow[t]{2}{*}{ GP } & $5.15(4.99)$ & $5.45(4.69)$ & $4.38(5.46)$ & $4.31(3.67)$ & $4.00(5.47)$ & $4.19(4.06)$ & $4.06(6.66)$ & $4.65(7.98)$ \\
\hline & \multicolumn{2}{|c|}{$n=38$} & \multicolumn{2}{|c|}{$n=129$} & \multicolumn{2}{|c|}{$n=263$} & \multicolumn{2}{|c|}{$n=215$} \\
\hline Specialist * & $.88(2.71)$ & $3.21(3.21)$ & $.99(2.79)$ & $3.26(4.34)$ & $.82(2.26)$ & $3.32(5.53)$ & $.87(2.15)$ & $3.40(4.12)$ \\
\hline
\end{tabular}

${ }^{*} \mathrm{p}<.05$ for specialist only

Table 3b: Rural Region

\begin{tabular}{|c|c|c|c|c|c|c|c|c|}
\hline & \multicolumn{2}{|c|}{ Morbidly Obese* } & \multicolumn{2}{|c|}{ Obese* } & \multicolumn{2}{|c|}{ Overweight* } & \multicolumn{2}{|c|}{ Normal* } \\
\hline & Self report & Actual & Self report & Actual & Self report & Actual & Self report & Actual \\
\hline & \multicolumn{2}{|c|}{$n=33$} & \multicolumn{2}{|c|}{$n=115$} & \multicolumn{2}{|c|}{$n=230$} & \multicolumn{2}{|c|}{$n=212$} \\
\hline & X (SD) & X (SD) & X (SD) & $\mathrm{X}(\mathrm{SD})$ & $\mathrm{X}(\mathrm{SD})$ & X (SD & $X(S D)$ & X (SD) \\
\hline \multirow[t]{2}{*}{ GP } & $5.64(4.83)$ & $6.51(6.63)$ & $5.10(6.03)$ & $3.84(4.12)$ & $4.27(5.80)$ & $3.54(3.68)$ & $4.02(4.55)$ & $3.58(3.30)$ \\
\hline & \multicolumn{2}{|c|}{$n=83$} & \multicolumn{2}{|c|}{$n=210$} & \multicolumn{2}{|c|}{$n=458$} & \multicolumn{2}{|c|}{$n=481$} \\
\hline Specialist * & $1.05(2.45)$ & $3.88(4.90)$ & $.99(2.69)$ & $3.44(4.01)$ & $.59(1.78)$ & $3.04(4.20)$ & $.44(.86)$ & $3.11(6.59)$ \\
\hline
\end{tabular}

${ }^{*} \mathrm{p}<.05$ for specialist only 
Table 4a: Eastern Health Region

\begin{tabular}{|c|c|c|c|c|c|c|c|c|}
\hline & \multicolumn{2}{|c|}{ Morbidly Obese* } & \multicolumn{2}{|c|}{ Obese* } & \multicolumn{2}{|c|}{ Overweight* } & \multicolumn{2}{|c|}{ Normal* } \\
\hline & Self report & Actual & Self report & Actual & Self report & Actual & Self report & Actual \\
\hline & \multicolumn{2}{|c|}{$\mathrm{n}=46$} & \multicolumn{2}{|c|}{$n=140$} & \multicolumn{2}{|c|}{$n=326$} & \multicolumn{2}{|c|}{$n=381$} \\
\hline & $X(S D)$ & $\mathrm{X}(\mathrm{SD})$ & $X(S D)$ & X (SD) & X (SD) & $X$ (SD & $\mathbf{X}$ (SD) & $\mathrm{X}(\mathrm{SD})$ \\
\hline GP & $5.74(4.86)$ & $7.35(5.55)$ & $5.06(6.28)$ & $4.67(3.61)$ & $4.40(5.73)$ & $4.55(4.22)$ & $4.34(6.98)$ & $4.57(4.52)$ \\
\hline & \multicolumn{2}{|c|}{$\mathrm{n}=46$} & \multicolumn{2}{|c|}{$n=129$} & \multicolumn{2}{|c|}{$n=319$} & \multicolumn{2}{|c|}{$n=346$} \\
\hline Specialist * & $1.33(3.62)$ & $3.77(3.53)$ & $1.29(3.22)$ & $3.83(4.38)$ & $.96(2.68)$ & $3.65(4.81)$ & $.91(2.13)$ & $3.88(6.19)$ \\
\hline
\end{tabular}

${ }^{*} \mathrm{p}<.05$ for specialist only

Table 4b: Central Health Region

\begin{tabular}{|c|c|c|c|c|c|c|c|c|}
\hline & \multicolumn{2}{|c|}{ Morbidly Obese* } & \multicolumn{2}{|c|}{ Obese* } & \multicolumn{2}{|c|}{ Overweight* } & \multicolumn{2}{|c|}{ Normal* } \\
\hline & Self report & Actual & Self report & Actual & Self report & Actual & Self report & Actual \\
\hline & \multicolumn{2}{|c|}{$\mathrm{n}=29$} & \multicolumn{2}{|c|}{$\mathrm{n}=61$} & \multicolumn{2}{|c|}{$\mathrm{n}=135$} & \multicolumn{2}{|c|}{$n=100$} \\
\hline & $\mathrm{X}$ (SD) & $\mathrm{X}$ (SD) & $\mathbf{X}(\mathrm{SD})$ & $\mathbf{X}(\mathrm{SD})$ & $\mathrm{X}(\mathrm{SD})$ & X (SD) & X (SD) & $\mathrm{X}(\mathrm{SD})$ \\
\hline \multirow[t]{2}{*}{ GP } & $5.45(3.87)$ & $6.06(6.08)$ & $4.41(4.15)$ & $3.77(3.47)$ & $4.07(4.14)$ & $3.73(2.98)$ & $4.00(4.82)$ & $5.45(3.87)$ \\
\hline & \multicolumn{2}{|c|}{$\mathrm{n}=30$} & \multicolumn{2}{|c|}{$n=67$} & \multicolumn{2}{|c|}{$n=135$} & \multicolumn{2}{|c|}{$\mathrm{n}=105$} \\
\hline Specialist * & $.50(1.07)$ & $4.33(5.65)$ & $1.21(2.96)$ & $3.91(5.54)$ & $.50(1.06)$ & $3.22(4.15)$ & $.69(1.81))$ & $3.02(3.55)$ \\
\hline
\end{tabular}

${ }^{*} \mathrm{p}<.05$ for specialist only

Table 4c: Western Health Region

\begin{tabular}{|c|c|c|c|c|c|c|c|c|}
\hline & \multicolumn{2}{|c|}{ Morbidly Obese* } & \multicolumn{2}{|c|}{ Obese* } & \multicolumn{2}{|c|}{ Overweight* } & \multicolumn{2}{|c|}{ Normal* } \\
\hline & Self report & Actual & Self report & Actual & Self report & Actual & Self report & Actual \\
\hline & \multicolumn{2}{|c|}{$\mathrm{n}=18$} & \multicolumn{2}{|c|}{$\mathrm{n}=56$} & \multicolumn{2}{|c|}{$\mathrm{n}=127$} & \multicolumn{2}{|c|}{$\mathrm{n}=137$} \\
\hline & $X(S D)$ & $\mathrm{X}(\mathrm{SD})$ & $\mathrm{X}$ (SD) & $\mathrm{X}(\mathrm{SD})$ & $\mathrm{X}(\mathrm{SD})$ & $\mathrm{X}(\mathrm{SD}$ & $\mathbf{X}(\mathrm{SD})$ & $\mathrm{X}(\mathrm{SD})$ \\
\hline \multirow[t]{2}{*}{ GP } & $5.28(6.47)$ & $5.82(4.04)$ & $4.43(4.37)$ & $4.74(4.94)$ & $4.19(6.17)$ & $4.11(4.44)$ & $3.82(5.74)$ & $5.28(6.47)$ \\
\hline & \multicolumn{2}{|c|}{$n=19$} & \multicolumn{2}{|c|}{$\mathrm{n}=52$} & \multicolumn{2}{|c|}{$\mathrm{n}=125$} & \multicolumn{2}{|c|}{$\mathrm{n}=124$} \\
\hline Specialist * & $.32(.82)$ & $2.63(2.18)$ & $.83(2.76)$ & $3.33(3.26)$ & $.71(2.05)$ & $3.55(7.94)$ & $.57(1.59)$ & $.32(.82)$ \\
\hline
\end{tabular}

${ }^{*} \mathrm{p}<.05$ for specialist only

Table 4d: Northern Health Region

\begin{tabular}{|c|c|c|c|c|c|c|c|c|}
\hline & \multicolumn{2}{|c|}{ Morbidly Obese ${ }^{1 *}$} & \multicolumn{2}{|c|}{ Obese* } & \multicolumn{2}{|c|}{ Overweight* } & \multicolumn{2}{|c|}{ Normal* } \\
\hline & Self report & Actual & Self report & Actual & Self report & Actual & Self report & Actual \\
\hline & \multicolumn{2}{|c|}{$\mathrm{n}=21$} & \multicolumn{2}{|c|}{$\mathrm{n}=55$} & \multicolumn{2}{|c|}{$\mathrm{n}=105$} & \multicolumn{2}{|c|}{$n=99$} \\
\hline & $X(S D)$ & $X(S D)$ & $\mathrm{X}(\mathrm{SD})$ & $X(S D)$ & $\mathrm{X}$ (SD) & $\mathrm{X}(\mathrm{SD}$ & $\mathbf{X}(\mathrm{SD})$ & $\mathrm{X}(\mathrm{SD})$ \\
\hline \multirow[t]{2}{*}{ GP } & $4.10(5.03)$ & $1.79(1.44)$ & $4.05(6.70)$ & $2.58(3.04)$ & $3.04(4.78)$ & $2.31(2.93)$ & $3.25(3.73)$ & $4.10(5.03)$ \\
\hline & \multicolumn{2}{|c|}{$n=26$} & \multicolumn{2}{|c|}{$\mathrm{n}=82$} & \multicolumn{2}{|c|}{$n=142$} & \multicolumn{2}{|c|}{$\mathrm{n}=121$} \\
\hline Specialist * & $1.19(2.59)$ & $2.34(2.01)$ & $.43(1.35)$ & $2.07(2.77)$ & $.50(1.12)$ & $1.96(2.40)$ & $.45(1.17)$ & $1.19(2.59)$ \\
\hline
\end{tabular}

* $p<.05$ for specialist only, ${ }^{1} p<.05$ for morbidly obese group and GP visits only 
APPENDIX F

Ethics Approval 


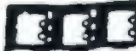

Human Investigation Committee

Research and Graduate Srudies

Freulty of Medicine

The Health Sciences Centre

November 16, 2005

Reference $\$ 05.197$

Ms. L. Twells

C/o Dr. B. Barrett

Patient Research Centre

$1^{\text {st }}$ Floor, Health Sciences Centre

Dear Ms. Twells:

This will acknowledge your correspondence dated November 11,2005, wherein you

- clarify issues and provide a letter granting permission to access the data from data guardians for your research study entitled "Health services utilization, associated factors, and direct cost of health services among obese vs. non-obese populations in Newfoundland and Labrador".

At the meeting held on October 13, 2005, the initial review date of this study, the Human Investigation Committee (HIC) agreed that the response could be reviewed by the Co-Chairs and, if found acceptable, full approval of the study be granted.

The Co-Chairs of the HIC reviewed your correspondence, and under the direction of the Committee, granted full approval of your research study. This will be reported to the full Human Investigation Committee, for their information at the meeting scheduled for November 24, 2005.

- Full approval has been granted for one year. You will be contacted for annual update before October 13, 2006.

Modifications of the protocol/consent are not permitted without prior approval from the Human Investigation Committee. Implementing changes in the protocol/consent without HIC approval may result in the approval of your research study being revoked, necessitating cessation of all related research activity. Request for modification to the protocol/consent must be outlined on an amendment form (available on the HIC website) and submitted to the HIC for review. 


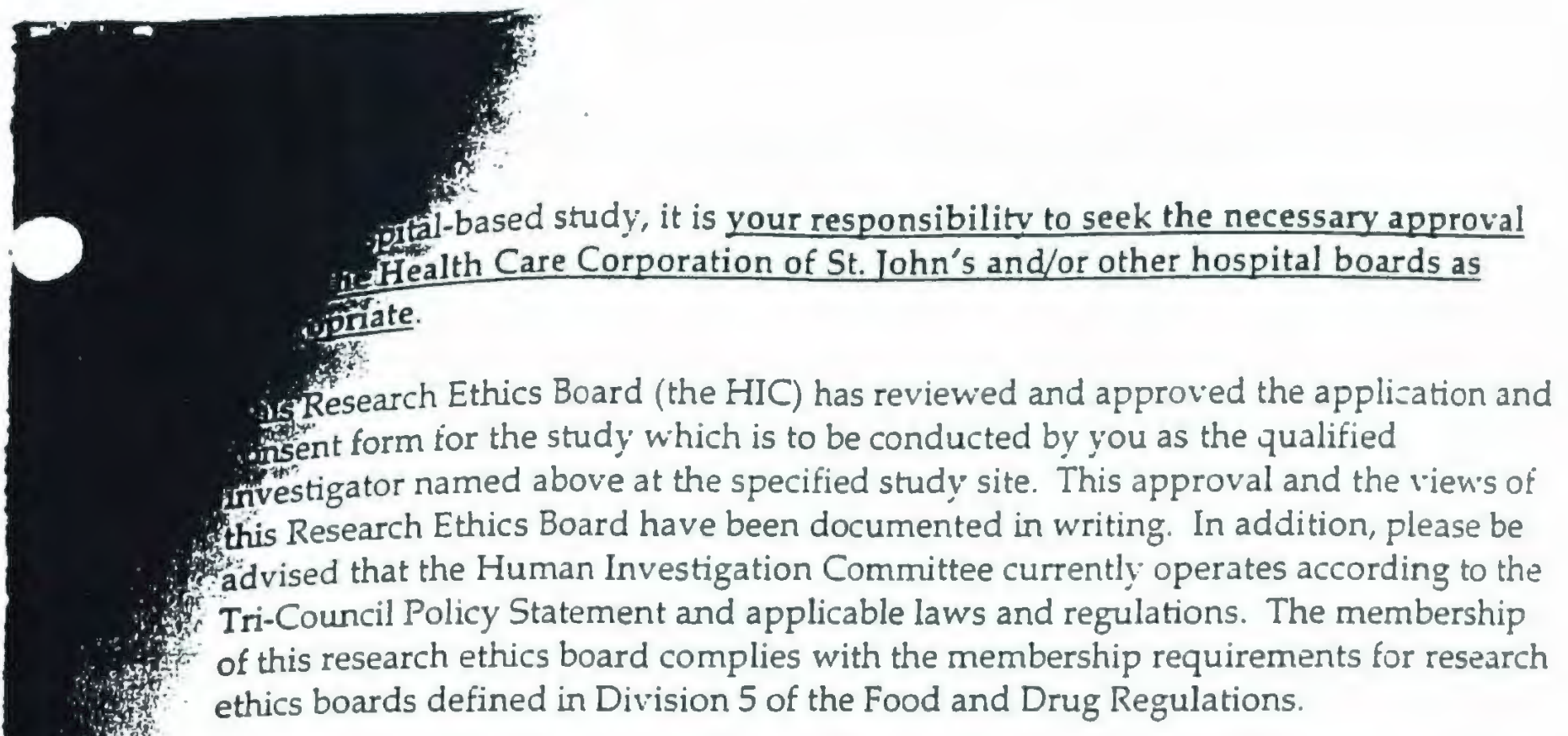

Notwithstanding the approval of the HIC, the primary responsibility for the ethical conduct of the investigation remains with you.

We wish you every success with your study.

Sincerely,

John D. Harnett, MD, FRCPC

Co-Chair

Human Investigation Committee

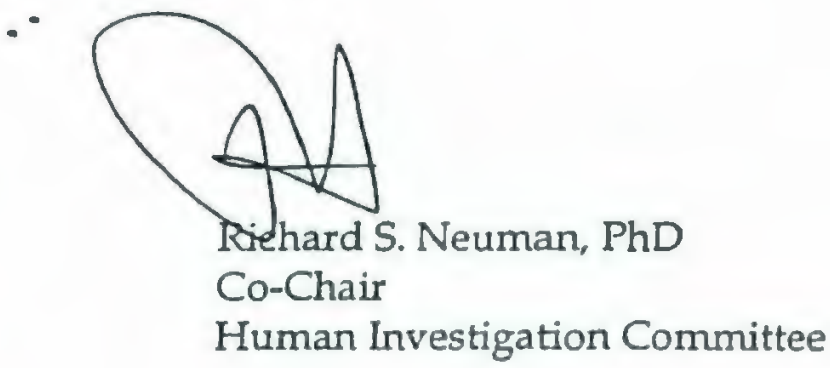

$\mathrm{JDH} ; \mathrm{RSN} \backslash \mathrm{jd}$

C Dr. C. Loomis, Vice-President (Research), MUN

M.r. W. Miller, Director of Planning \& Research, HCCS] 


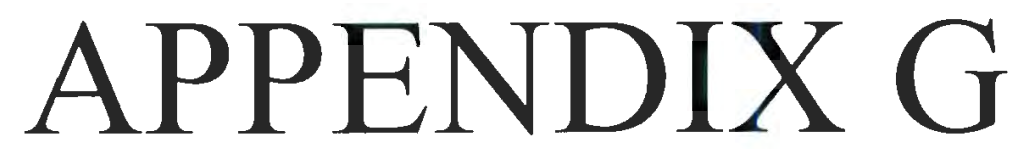

\section{Permission from the Department of Health and Community Services}




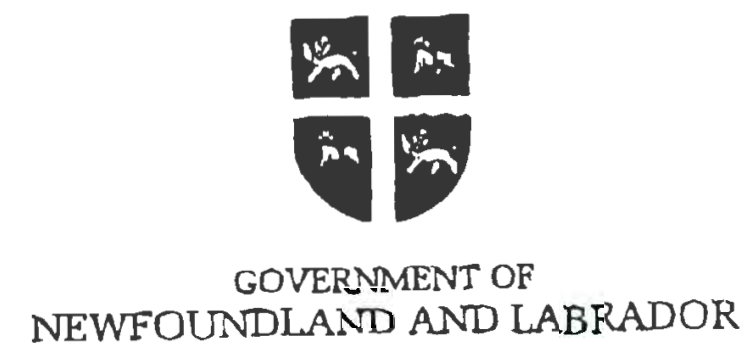

Department of Health and Community Services Planning and Evaluation Division

September 30, 2005

Laurie K. Twells

Division of Clinical Epidemiology

School of Medicine

Memorial University of Newfoundland

St. John's NL AlB 3V6

Dear Mrs. Twells:

I an writing in support of your study entitled "Health services utilization, associated factors, and direct cost of health services among obese vs. non-obese populations: Newfoundland and Labrador".

The Department of Health and Community Services, Newfoundland and Labrador recognizes the value in investigating "obesiry" in the province of New'foundland and Labrador, specifically as it relates to our strategic direction to improve population health iv our province. I grant you permission to use the CCHS 1.1 link file for this study and look forward to the fina! report.

Sincerely,

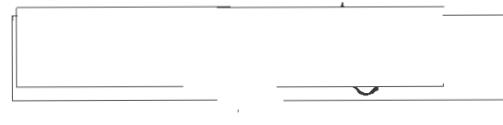

Regina Condy

Director

/cmt 


\section{APPENDIX H}

\section{Permission from the NL Centre for Health Information}




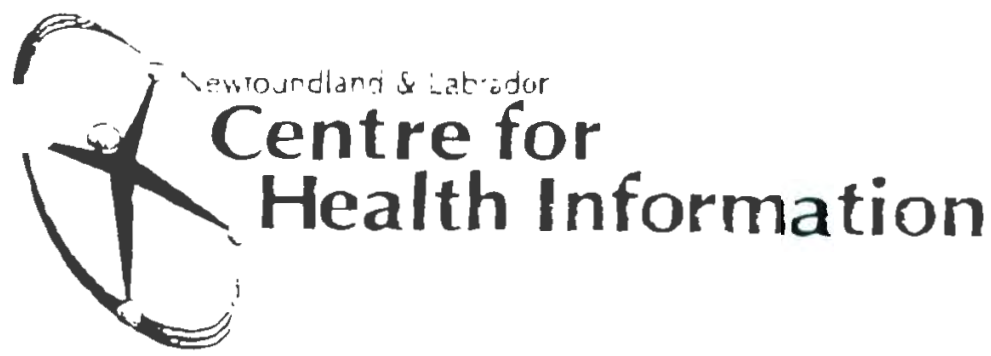

August 26, 2005

Laurie K. Twells

Division of Clinical Epidemiology

School of Medicine

Memorial University of Newfoundland

Dear Mrs. Twells:

I an writing in support of your study entitled "Health services utilization, associated factors, and direct cost of health services among obese vs. non-obese populations: Newfoundland and Labrador".

The Newfoundland and Labrador Centre for Health Information recognizes the value in investigating "obesity" in the province of Newfoundland and Labrador. I look forward to reading your final report.

Sincerely,

Steve O'Reilly

Chief Executive Ofincer

Crosbie Building

1 Crosbie Place

St. John's, NL ATB 3 Y 8

Telephone: (709) $757-2400$

Facsimile: (709) 757-2411
[- Registry Integrity Unit Harbour Lodge Nursing Home 86 Highroad South Carbnnear, NL A IY IA4 Telephone: (709) 945-5332:4/5 Facsimile: (709) 945-5340 
November $11^{\text {th }}, 2005$

Laurie K Twells

Clinical Epidemiology Unit

Faculty of Medicine

Memorial University of Newfoundland

300 Prince Philip Drive

St. John's, NL A1B 3 V6

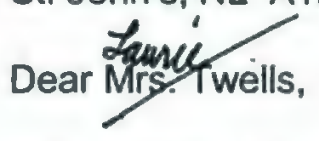

The Centre for Health Information, on behalf of the Department of Health and Community Services, is the guardian of three datasets that will be utilized for your study entitled "Health services utilization, associated factors, and direct cost of health services among obese vs. non-obese populations in Newfoundland and Labrador". By way of this letter, I approve limited access to data in the Canadian Community Health Survey (CCHS 1.1), the Medical Care Plan database (MCP) and the Clinical Database Management System (CDMS) for the purposes of your study. Please note that your access to this data will be limited to de-identified, anonymous information only.

The Newfoundland and Labrador Centre for Health Information supports your research on obesity in this province. I look forward to reading your final report.

Sincerely,

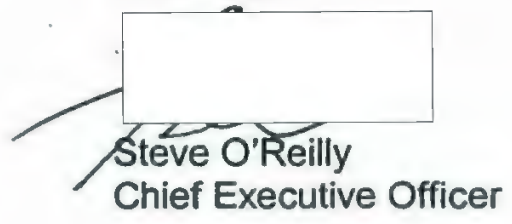




\section{APPENDIX I}

\section{Permission from the Medical Care Plan}


GOVERNMENT OF

NEWFOUNDLAND AND LABRADOR

Department of Health and Community Services

Audit and Claims Integrity

September 12, 2005

Laurie K. Twells

NL Centre for Applied Health Research

95 Bonaventure Avenue Suite 300

St. John's NL A1B 3V6

Dear Mrs. Twells:

I am writing in support of your study entitled "Health services utilization, associated factors, and direct cost of health services among obese vs. non-obese populations: Newfoundland and Labrador".

The Newfoundland Medical Care Plan (MCP) supports this project as we feel it would facilitate important research specific to obesity in this province. I wish you every success.

Sincerely,

TONY MAHER, C.A.

Executive Director 
APPENDIX J

\section{Poisson Models 1a-2c Actual Physician Utilization}


$>$ mode $11 a<-g 7 m$ (FFS_GPVS BMI_4CAT+DHHA_SEX+DHHA_AGE+EDUADH04+INCADIA + INST_NEW1 +SMKA_202+DHHA_MS+GEOHR2_R+PACADPAI+ALCADTYP+FAV_LRV1, weight=WTSA_S, data=newdata, fami $7 y=$ poisson)

Ca71 :

g7m(formula = FFS_GPVS BMI_4CAT + DHHA_SEX + DHHA_AGE + EDUADHO4 + INCADIAS + INST_NEW1 + SMKA_202 + DHHA_MS + GEOHR2_R + PACADPAI + ALCADTYP + FAV_LRV1, family = poisson, data = newdata, weights = WTSA_S)

Coefficients:

(Intercept)

BMI_4CAToverweight

BMI_4CATobese

BMI_4CATmorbid obesity

DHHA__SEXMale

DHHA_AGE

EDUADHO4SECONDARY GRAD.

EDUADH04OTHER POST-SEC.

EDUADHO4POST-SEC. GRAD.

EDUADHO4NOT STATED

INCADIA5LOWER MIDDLE

INCADIASMIDOLE

INCADIASUPPER MIDDLE

INCADIASHIGHEST

INCADIASNOT STATED

INST_NEW1Eastern

INST_NEWINorthern

INST_NEWIWEstern

SMKA_2020CCASIONALLY

SMKA_202NOT AT ALL

DHHA_MSCOMMON - LAW

DHHA_MSWIDOWED

DHHA_MSSEPARATED

DHHA_MSDIVORCED

DHHA_MSSINGLE

GEOHR2_Rurban

PACADPAIMODERATE

PACADPAIINACTIVE

PACADPAINOT STATED

ALCADTYPOCC. DRINKER

ALCADTYPFORMER DRINKER

ALCADTYPNEVER DRANK

0.3707696
ALCADTYPNEVER DRANK
FAV_LRV1< 5 serVings per day -0.0537634

-.-

signif. codes: 0 't*t*' 0.001

Estimate

0.0622520

$-0.0035596$

0.4269449

$-0.6051510$

0.0144896

$-0.1248718$

0.0008133

0.0514084

0.1748392

$-0.0379547$

$-0.2164000$

$-0.2617300$

$-0.1194637$

$-0.1585911$

0.1500639

$-0.7764669$

0.0345338

0.5041350

0.0139847

0.0844064

$-0.1618910$

0.2459173

0.4767913

0.1327632

0.1533851

$-0.1533460$

0.0263981

$-0.0115251$

0.3348397

0.2166951
Std. Error $z$ value $\operatorname{Pr}(>|z|)$

0.1899245

0.0568754

0.0691163

0.0871131

0.0531523

0.0023982

0.0838556

0.1195328

0.0688113

0.3816503

0.1061748

0.0999094

0.1033523

0.1190542

0.1500044

0.0586947

0.1160570

0.0652154

0.1150122

0.0574108

0.0861314

0.1539440

0.1806905

0.1170116

0.0800236

0.0503311

0.0864265

0.0732186

0.1654510

0.0595149

0.0722688

0.0856700

0.0555837

$-2.166$

$\begin{array}{ll}-0.967 & 0.33342\end{array}$
12.326

1.095

$-0.052$

$4.9019 .53 e-07$ \#*

$-11.385$

6.042

$-1.489$

0.007

0.747

0.458

$-0.357$

$-2.532$

$-1.003$

$-1.057$

2.557

$-6.690$

0.530

$4.3831 .17 \mathrm{e}-05$

0.2440 .80755

$\begin{array}{lll}0.980 & 0.32710\end{array}$

$-1.052$

1.361

0.29297

$4.0754 .61 e-05$ **

1.659

3.048

0.09711

$-1.774$

0.00231 *

0.361

0.07601

0.71844

$\begin{array}{lll}0.94447 & 070 & 0.94\end{array}$

$5.6261 .84 \mathrm{e}-08$ का को

2.9980 .00271 *त

$4.3281 .51 \mathrm{e}-05$ th

(Dispersion parameter for poisson family taken to be 1810.236 )

Number of Fisher scoring iterations: 6 
poisson_reg_utilization.txt

MODEL1B- ACTUAL GP VISITS (CC ONLY)

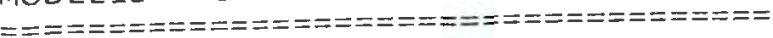

$>$ mode $11 b<-g 7 m$ (FFS_GPVS CRNC_TOT+DHHA_SEX+DHHA_AGE+EDUADH04+INCADIA 5+INST_NEW1+ SMKA_202+DHHA_MS+GEOHR2_R+PACADPAI+ALCADTYP+FAV_LRV1, weight=WTSA_S, data $=$ newdata, fami $1 \mathrm{y}=$ poisson)

Ca11:

$\mathrm{g} 7 \mathrm{~m}$ (Formula = FFS_GPVS CRNC_TOT + DHHA_SEX + DHHA_AGE + EDUADHO4 + INCADIA5 + INST_NEW1 + SMKA_202 + DHHA_MS + GEOHR2_R + PACADPAI +

ALCADTYP + FAV_LRV1, family = poisson, data $=$ newdata, weights $=$ WTSA_S $)$

Coefficients:

(Intercept)

CRNC_TOT

DHHA_SEXmale

DHHA_AGE

EDUADH04SECONDARY GRAD.

EDUADH04OTHER POST-SEC.

EDUADH04POST-SEC. GRAD.

EDUADHO4NOT STATED

INCADIA5LOWER MIDDLE

INCADIA5MIDDLE

INCADIA5UPPER MIDDLE

INCADIASHIGHEST

INCADIASNOT STATED

INST_NEW1Eastern

INST_NEW1Northern

INST_NEWIWEStern

SMKA_202OCCASIONALLY

SMKA_202NOT AT ALL

DHHA_MSCOMMON - LAW

DHHA MSWIDOWED

DHHA_MSSEPARATED

DHHA_MSDIVORCED

DHHA_MSSINGLE

GEOHR2_RUrban

PACADPAIMODERATE

PACADPAI INACTIVE

PACADPAINOT STATED

ALCADTYPOCC. DRINKER

ALCADTYPFORMER DRINKER

ALCADTYPNEVER DRANK

FAV_LRV $1<5$ servings per day

Ang per day -0.008733

---

Signif. codes: 0 '*k'0.001 0.01

Estimate std. Error $z$ value $\operatorname{Pr}(>|z|)$

$2.357994 \quad 0.179413 \quad 13.143<2 \mathrm{e}-16$ *

$0.2103270 .01243816 .911<2 \mathrm{e}-16$ «

$-0.540167$

0.005074

$-0.077421$

$-0.014665$

0.084743

0.108242

$-0.040992$

$-0.127599$

$-0.158789$

$-0.019311$

$-0.135312$

0.173829

$-0.798695$

0.066543

0.522323

0.038385

0.064113

$-0.068102$

0.285127

0.492934

0.109758

0.143785

$-0.193277$

$-0.019497$

$-0.084802$

0.309003

0.168682

0.311200

0.049641

0.002376

0.078864

0.112676

0.064648

0.357658

0.099599

0.093511

0.096583

0.110749

0.139680

0.054895

0.108945

0.061332

0.107924

0.053832

0.080550

0.144030

0.168633

0.110432

0.076402

0.047206

0.080829

0.068719

0.155336

0.055671

0.067830

0.080339

0.052128

$10.881<2 \mathrm{e}-16$

2.1360 .032687

$-0.9820 .326245$

$\begin{array}{lll}-0.130 & 0.896449\end{array}$

$\begin{array}{lll}1.311 & 0.189911\end{array}$

$0.303 \quad 0.762164$

$\begin{array}{lll}-0.412 & 0.680654\end{array}$

$\begin{array}{lll}-1.365 & 0.172399\end{array}$

$-1.6440 .100164$

$\begin{array}{lll}-0.174 & 0.861578\end{array}$

$-0.9690 .332683$

3.1670 .001542 th

$-7.3312 .28 \mathrm{e}-13$ w.

1.0850 .277936

$4.8401 .30 \mathrm{e}-06$.

0.7130 .475812

$0.796 \quad 0.426069$

$-0.4730 .636331$

1.6910 .090873

$4.4648 .06 \mathrm{e}-06$ i*

1.4370 .150833

3.0460 .002320 *

-2.3910 .016793 *

$-0.2840 .776623$

$-0.5460 .585117$

$5.5502 .85 e-08$ th

2.4870 .012889 "

$\begin{array}{lll}3.874 & 0.000107\end{array}$

$-0.1680 .866956$

(Dispersion parameter for poisson family taken to be 1590.012 )

Number of Fisher scoring iterations: 6

Page 2 
poisson_reg_utilization.txt

MODELIC - ACTUAL GP VISITS (BMI+CC)

$>$

mode $11 c<-g 7 m$ (FFS_GPVS BMI_4CAT+CRNC_TOT+DHHA_SEX+DHHA_AGE+EDUADH04+INCADIA5+INST_NEW 1+SMKA_202+DHHA_MS+GEOHR2_R+PACADPAI+ALCADTYP+FAV_LRV1, weight=WTSA_S, data=newdata, fami $7 y=$ poisson)

cal1:

g7m(formula = FFS_GPVS BMI_4CAT + CRNC_TOT + DHHA_SEX + DHHA_AGE + EDUADH04 + INCADIA5 + INST_NEW1 + SMKA_202 + DHHA_MS + GEOHR2_R + PACADPAI + ALCADTYP + FAV_LRV1, famiTy = poisson, data = newdata, weights = WTSA_S )

Coefficients:

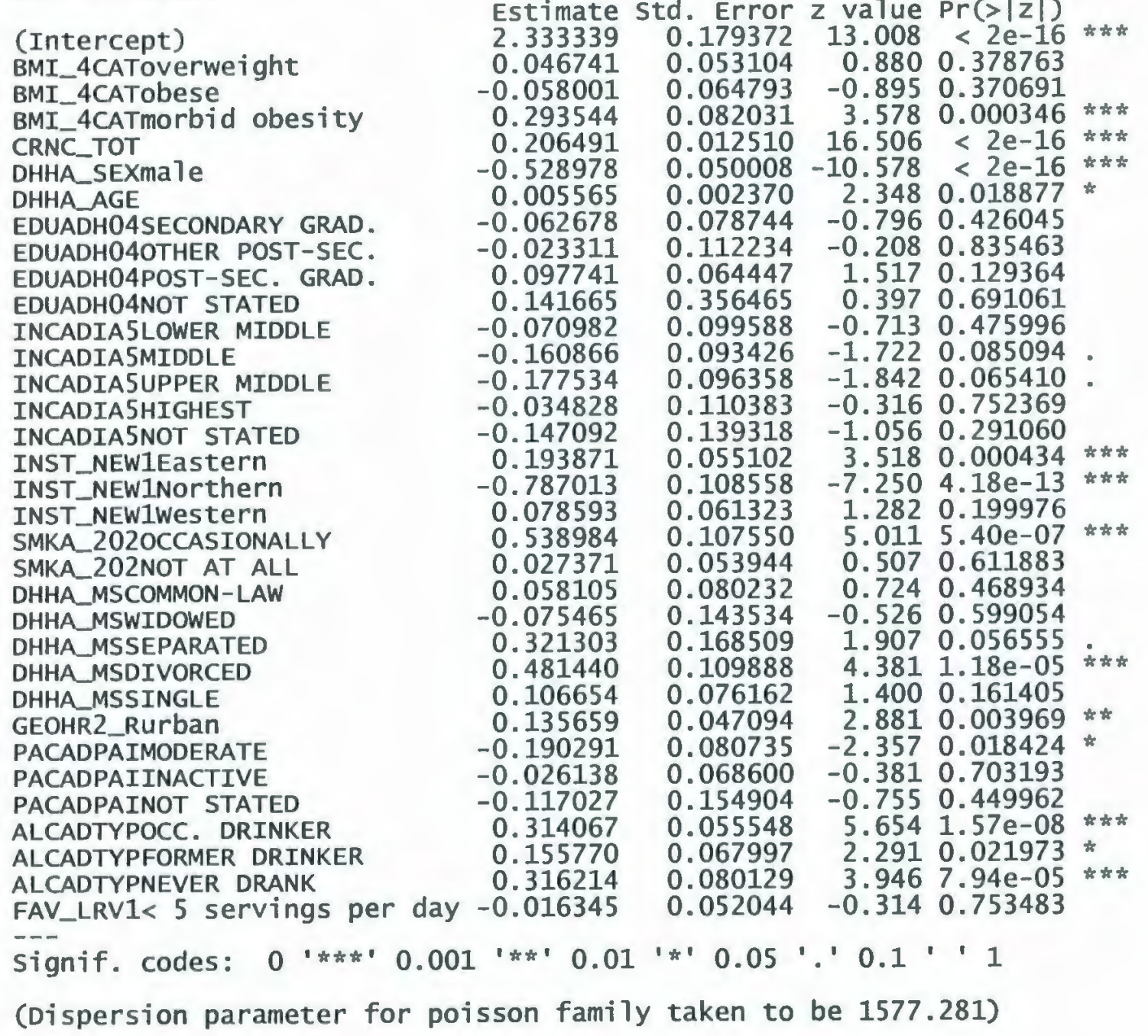

Number of Fisher Scoring iterations: 6 
MODEL2A- ACTUAL VISITS WITH SPECIALIST (BMI ONLY)

$>$

mode $12 \mathrm{a}<-\mathrm{g} 7 \mathrm{~m}$ (FFS_SPEC BMI_4CAT+DHHA_SEX+DHHA_AGE+EDUADH04+INCADIA5+INST_NEW1+SMKA_20 2+DHHA_MS+GEOHR2_R+PACADPAI+ALCADTYP+FAV_LRV1, weight=WTSA_S, data=newdata, fami $1 y=$ poisson)

Ca $11:$

glm (formula = FFS_SPEC BMI_4CAT + DHHA_SEX + DHHA_AGE + EDUADH04 + INCADIA5 + INST_NEW1 + SMKA_ $202+$ DHHA_MS + GEOHR2_R + PACADPAI + ALCADTYP + FAV_LRV1, family = poisson, data = newdata, weights = WTSA_S)

coefficients:

(Intercept)

BMI_4CAToverweight

BMI_4CATobese

BMI_4CATmorbid obesity

DHHA_SEXmale

DHHA.AGE

EDUADH04SECONDARY GRAD, EDUADH04OTHER POST-SEC.

EDUADH04POST-SEC. GRAD.

EDUADHOANOT STATED

INCADIASLOWER MIDDLE

INCADIASMIDDLE

INCADIASUPPER MIDDLE

INCADIASHIGHEST

INCADIASNOT STATED

INST NEWIEastern

INST_NEWINOr thern

INST_NEWIWEStern

SMKA_2020CCASIONALLY

SMKA_202NOT AT ALL

DHHA_MSCOMMON-LAW

DHHA_MSWIDOWED

DHHA_MSSEPARATED

DHHA_MSDIVORCED

DHHA MSSINGLE

GEOHR2_RU rban

PACADPATMODERATE

PACADPAIINACTIVE

PACADPAINOT STATED

ALCADTYPOCC. DRINKER

ALCADTYPFORMER DRINKER

ALCADTYPNEVER DRANK

FAV_LRVI< 5 servings per day -0.092140

Signif. codes: 0 "t*t 0.001 'tक' 0.01

(Dispersion parameter for poisson family taken to be 1934.381)

0.287884

0.113142

*' 0.05 '.'0.1' 1
Estimate std. Error $z$ value $\operatorname{Pr}(>|z|)$

$\begin{array}{lllll}1.523506 & 0.233208 & 6.533 & 6.45 \mathrm{e}-11\end{array}$ औ虾

$\begin{array}{llll}0.094175 & 0.068473 & 1.375 & 0.169024\end{array}$

$\begin{array}{llll}-0.021141 & 0.083507 & -0.253 & 0.800139\end{array}$

$\begin{array}{lllll}0.185704 & 0.116096 & 1.600 & 0.109693\end{array}$

$-0.316710$

0.029248

$-0.235502$

$-0.185489$

$-0.094227$

$-0.224637$

$-0.321848$

$-0.364202$

$-0.368614$

$-0.396563$

$-0.420483$

$-0.200685$

$-0.271299$

0.161290

$-0.024140$

$-0.384762$

0.955212

$-0.055880$

0.058892

0.121799

0.357911

0.361342

0.140784

0.038034

0.063359

0.002946

0.099136

0.151695

0.080464

0.492539

0.127870

0.116731

0.120586

0.143434

0.183128

0.070413

0.118626

0.078527

0.191482

0.070740

0.114059

0.191123

0.171135

0.177569

0.096896

0.060274

0.098469

0.184046

0.074879

0.088570

0.097943

$-4.9995,77 e-07$ *t th

$9.928<2 e-16$ *t

$-2.3760 .017523$

$-1.2230 .221413$

$\begin{array}{lll}-1.171 & 0.241581\end{array}$

$\begin{array}{lll}-0.456 & 0.648333\end{array}$

-2.5170 .011836 *

-3.1200 .001808 का

-3.0570 .002237 tक

-2.7650 .005696 * *

-2.2960 .021669 *

-2.8500 .004371 st

$-4.3441 .40 e-05$ tक

-2.8940 .003798 क

$\begin{array}{lll}-1.417 & 0.156530\end{array}$

2.2800 .022604 *

$\begin{array}{lll}-0.212 & 0.832384\end{array}$

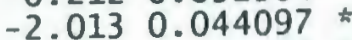

$5.5822 .38 \mathrm{e}-08$ से

$\begin{array}{lll}-0.315 & 0.752994\end{array}$

2.9710 .002968 सर

0.9770 .328529

1.0770 .281694

3.635 0.000278 करत

1.9630 .049609 *

1.8800 .060086 .

0.4290 .667615

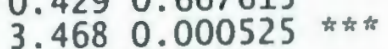

0.066791

$-1.3800 .167734$

Number of Fisher scoring iterations: 6

Page 4 
MODEL2B - ACTUAL VISITS WITH SPECIALIST (CC ONLY)

mode $12 b<-g 7 m$ (FFS_SPEC CRNC_TOT+DHHA_SEX+DHHA_AGE+EDUADH04+INCADIA5+INST_NEW1+SMKA_20 2+DHHA_MS +GEOHR2_R+PACADPAI+ALCADTYP+FAV_LRV1, weight=WTSA_S, data=newdata, fami $7 y=$ poisson)

Ca77:

g1m(formula = FFS_SPEC CRNC_TOT + DHHA_SEX + DHHA_AGE + EDUADHO4 +

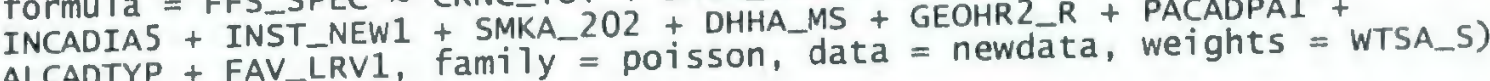

Coefficients:
(Intercept)

CRNC_TOT

DHHA_SEXmaTe

DHHA_AGE

EDUADH04SECONDARY GRAD.

EDUADHO4OTHER POST-SEC.

EDUADH04POST-SEC. GRAD.

EDUADHO4NOT STATED

INCADIASLOWER MIDDLE

INCADIASMIDDLE

INCADIASUPPER MIDDLE

INCADIASHIGHEST

INCADIASNOT STATED

INST_NEWIEastern

INST_NEW1Nor thern

INST_NEWIWEstern

SMKA_2020CCASIONALLY

SMKA_202NOT AT ALL

DHHA_MSCOMMON-LAW

DHHA_MSWIDOWED

DHHA_MSSEPARATED

DHHA_MSDIVORCED

DHHA_MSSINGLE

GEOHR2_RUrban

PACADPAIMODERATE

PACADPAIINACTIVE

PACADPAINOT STATED

ALCADTYPOCC. DRINKER

ALCADTYPFORMER DRINKER

ALCADTYPNEVER DRANK

$\begin{array}{lr}\text { ALCADTYPNEVER DRANK } & 0.27509 \\ \text { FAV_LRV } 1<5 \text { servings per day } & -0.03147\end{array}$

0.22869

$-0.22987$

0.01946

$-0.16467$

$-0.23604$

$-0.05727$

$-0.27441$

$-0.34240$

$-0.27257$

$-0.22236$

$-0.23777$

$-0.42800$

$-0.15767$

$-0.52757$

$-0.18176$

0.17011

$-0.04877$

$-0.26835$

0.96036

$-0.01986$

0.27526

0.02795

0.06732

0.27870

0.27150

0.13043
Estimate std. Error $z$ value $\operatorname{Pr}(>|z|)$

1.49514

$-0.15185$

$-0.03940$

signif. codes: $0^{\prime * t *} 0.001^{\prime * *} 0.01$
. 22033

0.01416

0.00292

0.09364

0.14290

0.07573

0.46218

0.12052

0.11000

0.11330

0.13414

0.17073

0.06599

0.11161

0.07405

0.17983

0.06658

0.10692

0.17900

0.15986

0.16713

0.09263

0.05662

0.10625

0.09267

0.17266

0.07021

0.08378

0.09204

0.06284
$6.7861 .15 \mathrm{e}-11$ \#*

$16.150<2 \mathrm{e}-16$ \#*

-3.8710 .000108 औ办

$6.6652 .64 \mathrm{e}-11$ सक स

-1.7590 .078657 .

-1.6520 .098577 .

$\begin{array}{lll}-0.756 & 0.449471\end{array}$

$\begin{array}{lll}-0.594 & 0.552694\end{array}$

$\begin{array}{lll}-2.841 & 0.004498\end{array}$

$\begin{array}{lll}-2.478 & 0.013215\end{array}$ *

-1.9630 .049698 *

-1.7730 .076297 .

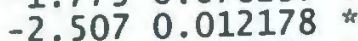

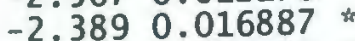

$-4.7272 .28 \mathrm{e}-06$ * $*$ t

-2.0500 .040321 *

$-1.0110 .312133$

2.5550 .010623 *

$\begin{array}{lll}-0.456 & 0.648279\end{array}$

$\begin{array}{lll}-1.499 & 0.133835\end{array}$

$6.0071 .89 \mathrm{e}-09$ *t*

2.9720 .002962 * *

0.4940 .621603

0.6340 .526320

3.0070 .002635 * *

1.5730 .115831

1.8580 .063222 .

\begin{tabular}{l}
-0.4700 .638176 \\
\hline
\end{tabular}

2.9890 .002801 *t

$-0.5010 .616484$ $\begin{array}{lll}-0.119 & 0.905409\end{array}$

(Dispersion parameter for poisson family taken to be 1702.554 )

Number of Fisher scoring iterations: 6 
poisson_reg_utilization.txt

MODEL2C- ACTUAL VISITS SPECIALIST(BMI+CC)

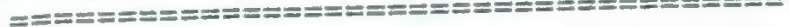

$2>$

mode $12 \mathrm{C}<-\mathrm{g} 1 \mathrm{~m}$ (FFS_SPEC BMI_4CAT+CRNC_TOT+DHHA_SEX+DHHA_AGE+EDUADH04+INCADIAS+INST_NEW 1+SMKA_202+DHHA_MS+GEOHR2_R+PACADPAI+ALCADTYP+FAV_LRV1, weight=WTSA_S, data=newdata, fami $1 y=$ poisson)

cal1:

glm (formula $=$ FFS_SPEC $\sim$ BMI_4CAT + CRNC_TOT + DHHA_SEX + DHHA_AGE + EDUADH04 + INCADIA5 + INST_NEW1 + SMKA_202 + DHHA_MS + GEOHR2_R + PACADPAI + ALCADTYP + FAV_LRV1, family = poisson, data = newdata, weights $=$ WTSA_S )

coefficients:

(Intercept)

BMI_4CAToverwejght

BMI_4CATobese

BMI_4CATmorbid obesity

CRNC_TOT

DHHA_SEXmale

DHHA AGE

EDUADH04SECONDARY GRAD.

EDUADH04OTHER POST-SEC.

EDUADH04POST-SEC. GRAD.

EDUADH04NOT STATED

INCADIASLOWER MIDDLE

INCADIA5MIDDLE

INCADIASUPPER MIDDLE

INCADIA5HIGHEST

INCADIASNOT STATED

INST_NEW1Eastern

INST_NEW1Nor thern

INST_NEWIWestern

SMKA_2020CCASIONALLY

SMKA_202NOT AT ALL

DHHA_MSCOMMON-LAW

DHHA_MSWIDOWED

DHHA_MSSEPARATED

DHHA_MSDIVORCED

DHHA_MSSINGLE

GEOHR2_RUrban

PACADPAIMODERATE

PACADPAIINACTIVE

PACADPAINOT STATED

ALCADTYPOCC. DRINKER

ALCADTYPFORMER DRINKER

ALCADTYPNEVER DRANK

$\begin{array}{lr}\text { ALCADTYPNEVER DRANK } & 0.272835 \\ \text { FAV_LRV } 1<5 \text { servings per day }-0.031127\end{array}$

$-0.049522$

$-0.251503$

$-0.355817$

$-0.281827$

$-0.235298$

$-0.258584$

$-0.427492$

$-0.150353$

$-0.522894$

$-0.154314$

$-0.175646$

0.178549

$-0.050715$

$-0.261826$

0.990619

$-0.025671$

0.279371

0.025679

0.060597

0.275694

0.257949

0.134391
Estimate std. Error $z$ value $\operatorname{Pr}(>|z|)$

$\begin{array}{rrrr}1.482100 & 0.221556 & 6.690 & 2.24 \mathrm{e}-11\end{array}$

$\begin{array}{llll}0.083906 & 0.064304 & 1.305 & 0.191955\end{array}$

$\begin{array}{lllll}-0.097490 & 0.078781 & -1.237 & 0.215909\end{array}$

$\begin{array}{llll}0.005933 & 0.110699 & 0.054 & 0.957256\end{array}$

$0.231293 \quad 0.014365$

$-0.227690 \quad 0.060039$

$0.019321 \quad 0.002923$

$-0.155650 \quad 0.093715$

$-0.236686 \quad 0.142917$

0.075883

0.462175

0.120798

0.110296

0.113432

0.134410

0.170747

0.066328

0.111564

0.074260

0.179686

0.067104

0.106876

0.178977

0.160390

0.167066

0.093035

0.056727

0.106282

0.092670

0.172977

0.070178

0.084211

0.092017

$16.101<2 \mathrm{e}-16$ *t

-3.7920 .000149 *t

$6.6093 .86 \mathrm{e}-11$ k屯

-1.6610 .096736 .

-1.6560 .097700 .

$-0.6530 .514004$

$-0.5440 .586323$

-2.9460 .003224 * *

-2.5550 .010613 *

-2.0740 .038046 *

-1.9240 .054375 .

-2.5040 .012292 *

$-2.2670 .023402$

$-4.6872 .77 \mathrm{e}-06$ * *

-2.0780 .037708 *

$-0.9780 .328313$

2.6610 .007796 *

$-0.4750 .635127$

$-1.4630 .143493$

$6.1766 .56 \mathrm{e}-10$ औt

$-0.1540 .877878$

3.0030 .002675 th

0.4530 .650787

0.5700 .568574

2.9750 .002930 **

1.4910 .135902

1.9150 .055492

$-0.4220 .672683$

2.9650 .003026 *

$\begin{array}{lll}0.062892 & -0.495 & 0.620647\end{array}$

signif. codes: 0 " 0.001 " 0.01

(Dispersion parameter for poisson family taken to be 1699.571 )

Number of Fisher scoring iterations: 6 
APPENDIX K

\author{
Poisson Models 3a-5c \\ Actual Hospital Utilization
}


poisson_reg_utilization.txt

MODEL3A: ACTUAL ACUTE VISITS (BMI ONLY)

$>$

mode $13 a<-g 1 m$ (ACUTE_VS BMI_4CAT+DHHA_SEX+DHHA_AGE+EDUADH04+INCADIAS+INST_NEW1+SMKA_20 2+DHHA_MS+GEOHR2_R+PACADPAI+ALCADTYP+FAV_LRV1, weight=WTSA_S, data=newdata, fami $7 y=$ poisson)

$>$ summary (mode18a, dispersion=mode18a\$deviance/mode18a\$df.residua1)

glm (formula = ACUTE_VS $\sim$ BMI_4CAT + DHHA_SEX + DHHA_AGE + EDUADHO4 + INCADIA5 + INST_NEW1 + SMKA_202 + DHHA_MS + GEOHR2 R + PACADPAI + WTSA_S )
ALCADTYP + FAV LRV1, family $=$ poisson, data = newdata, weights $=$ WTSA

Coefficients:

(Intercept)

BMI_4CAToverweight

BMI 4CATobese

BMI_4CATmorbid obesity

DHHA_SEXma Te

DHHA_AGE

EDUADH04SECONDARY GRAD.

EDUADH04OTHER POST-SEC.

EDUADH04POST-SEC. GRAD .

EDUADHO4NOT STATED

INCADIA5LOWER MIDDLE

INCADIA5MIDDLE

INCADIA5UPPER MIDDLE

INCADIA SHIGHEST

INCADIASNOT STATED

INST_NEW1Eastern

INST_NEW1Northern

INST_NEWIWestern

SMKA_2020CCASIONALLY

SMKA_202NOT AT ALL

DHHA_MSCOMMON-LAW

DHHA_MSWIDOWED

DHHA_MSSEPARATED

DHHA_MSDIVORCED

DHHA_MSSINGLE

GEOHR2 RUrban

PACADPAIMODERATE

PACADPAIINACTIVE

PACADPAINOT STATED

ALCADTYPOCC. DRINKER

ALCADTYPFORMER DRINKER

ALCADTYPNEVER DRANK

ALCADTYPNEVER DRANK

$-0.462239$
Estimate std. Error $z$ value $\operatorname{Pr}(>|z|)$

Estimate

$-0.628609$

$-0.025819$

$-0.191561$

0.257252

$-0.518765$

0.001377

$-0.086984$

0.068674

0.088915

$-0.937840$

0.040146

$-0.386334$

$-0.328858$

$-0.787049$

$-0.793375$

$-0.407040$

0.575277

$-0.036480$

$-0.326842$

0.051245

0.101500

0.478670

0.129734

0.297985

$-0.072176$

$-0.050029$

0.195429

0.610537

0.420308

0.346611

0.532961
0.337349

0.102003

0.127179

0.154234

0.096210

0.004294

0.151568

0.200302

0.124028

1.021406

0.185069

0.181694

0.184471

0.235585

0.289299

0.115717

0.126556

0.118094

0.280171

0.100721

0.148666

0.338490

0.296316

0.259051

0.133675

0.089913

0.161972

0.137120

0.234248

0.107932

0.131728

0.145956

0.100067 $\begin{array}{lll}-1.863 & 0.062409\end{array}$

$-0.2530 .800173$

$\begin{array}{lll}-1.506 & 0.132009\end{array}$

1.6680 .095329

$-5.3926 .97 e-08$ क巾

0.3210 .748530

$\begin{array}{lll}-0.574 & 0.566041\end{array}$

0.3430 .731708

0.7170 .473441

$\begin{array}{lll}-0.918 & 0.358522\end{array}$

$0.217 \quad 0.828266$

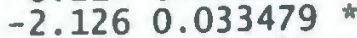

$-1.7830 .074635$

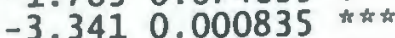

-2.7420 .006099 *t

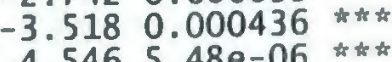

$-0.3090 .757394$

$\begin{array}{lll}-1.167 & 0.243381\end{array}$

0.5090 .610903

$\begin{array}{lll}0.683 & 0.494771\end{array}$

$\begin{array}{lll}0.1 .366 & 0.172067\end{array}$

1.6150 .106223

0.5010 .616508

2.2290 .025802 *

$-0.3090 .757419$

1.4250 .154088

$2.606 \quad 0.009151$ ks

$3.8949 .85 \mathrm{e}-05$ t虾

2.6310 .008507 th

3.6520 .000261 kt

$\begin{array}{lll}-1.305 & 0.191961\end{array}$ $\begin{array}{lll}-0.803 & 0.422127\end{array}$

Signif. codes: $0^{\prime * *} 0.001$ '*t 0.01

$1: 0.05 \cdot 0.1 \cdot 1$

(Dispersion parameter for poisson family taken to be 144.0259 )

Number of Fisher scoring iterations: 6 
poisson_reg_utilization.txt

MODEL 3B: ACTUAL ACUTE VISITS (CC ONLY)

$>$

mode $13 b<-g 7$ m (ACUTE_VS CRNC_TOT+DHHA_SEX+DHHA_AGE+EDUADH04+INCADIA5+INST_NEW1+SMKA_20 2+DHHA_MS+GEOHR2_R+PACADPAI+ALCADTYP+FAV_LRV1, weight=WTSA_S, data=newdata, fami $y=$ =poisson)

Ca77: INCADIA5 + INST_NEW1 + SMKA_202 + DHHA_MS + GEOHR2 R + PACADPAI +
ALSA_S

Coefficients:

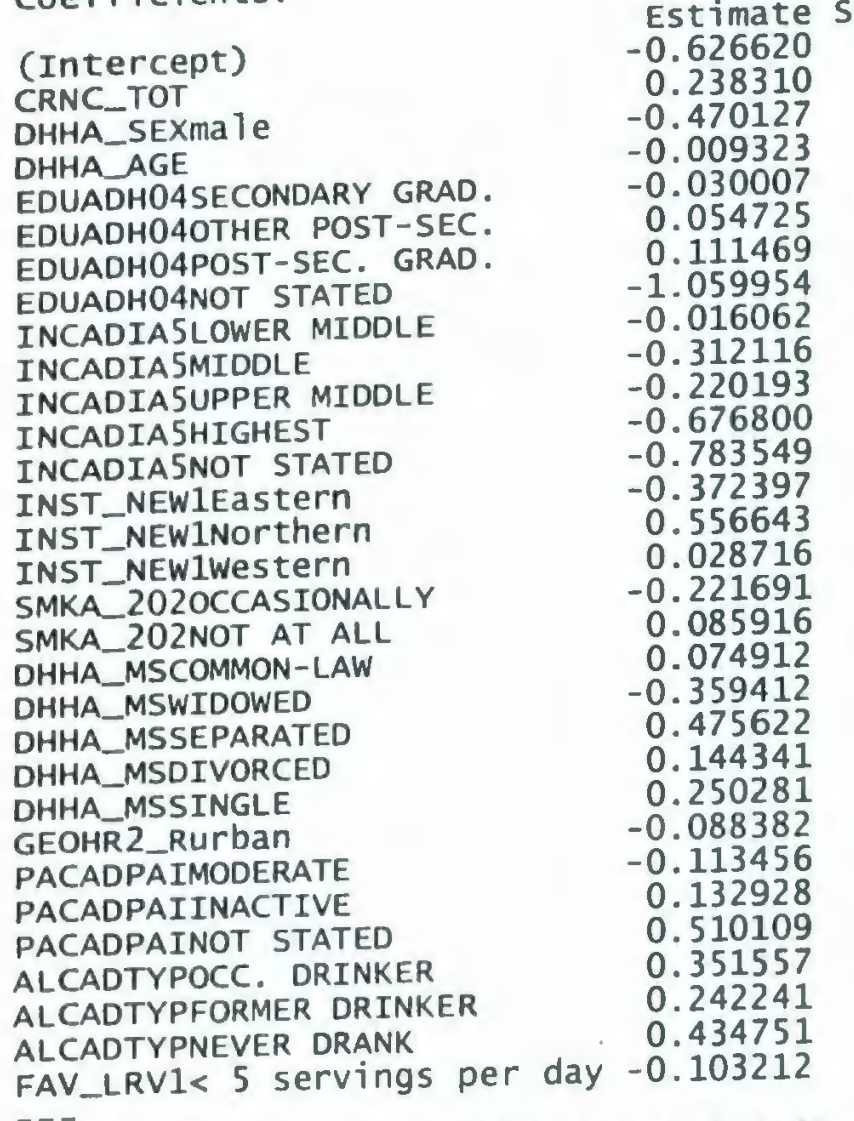

Std. Error $z$ value $\operatorname{Pr}(>|z|)$

$0.329972-1.8990 .057563$

$0.022611 \quad 10.540<2 e-16$ क a $^{2}$

$0.092926-5.0594 .21 \mathrm{e}-07$ th

$0.004455-2.0930 .036384$ *

$0.148045-0.2030 .839376$

$\begin{array}{lll}0.195698 & 0.280 & 0.779753\end{array}$

$\begin{array}{llll}0.121399 & 0.918 & 0.358511\end{array}$

$\begin{array}{llll}0.993684 & -1.067 & 0.286111\end{array}$

$\begin{array}{llll}0.180560 & -0.089 & 0.929118\end{array}$

$\begin{array}{llll}0.176529 & -1.768 & 0.077049\end{array}$

$\begin{array}{llll}0.179058 & -1.230 & 0.218799\end{array}$

$0.228345 \quad-2.9640 .003037$ *

$0.280522-2.7930 .005219$ **

$0.112712-3.3040 .000953$ क*

$0.123625 \quad 4.5036 .71 \mathrm{e}-06$ t *

$\begin{array}{lll}0.115105 & 0.249 & 0.802990\end{array}$

$\begin{array}{lll}0.272997 & -0.812 & 0.416755\end{array}$

$\begin{array}{llll}0.098338 & 0.874 & 0.382290\end{array}$

$\begin{array}{lll}0.143965 & 0.520 & 0.602820\end{array}$

$\begin{array}{lll}0.329134 & -1.092 & 0.274837\end{array}$

$\begin{array}{lll}0.287056 & 1.657 & 0.097541\end{array}$

$0.252897 \quad 0.5710 .568169$

$\begin{array}{llll}0.132509 & 1.889 & 0.058920\end{array}$

$\begin{array}{llll}0.087642 & -1.008 & 0.313240\end{array}$

$\begin{array}{lll}0.157543 & -0.720 & 0.471427\end{array}$

$\begin{array}{lll}0.133625 & 0.995 & 0.319842\end{array}$

$0.228550 \quad 2.2320 .025620$ *

0.1051113 .3450 .000824 th th

$0.128471 \quad 1.8860 .059353$.

$0.142036 \quad 3.0610 .002207$ क巾

$\begin{array}{llll}0.097134 & -1.063 & 0.287975\end{array}$

Signif. codes: 0 "ta' 0.001 "*t 0.01 ' $0.05 \cdot 0.1$ ' 1

(Dispersion parameter for poisson family taken to be 136.3736 )

Number of Fisher scoring iterations: $\mathbf{6}$ 
poisson_reg_utilization.txt

MODEL 3C: ACTUAL ACUTE VISITS (BMI +CC)

$>$

mode $13 \mathrm{c}<-\mathrm{g} 7 \mathrm{~m}$ (ACUTE_VS BMI_4CAT+CRNC_TOT+DHHA_SEX+DHHA_AGE+EDUADH04+INCADIA5+INST_NEW 1+SMKA_202+DHHA_MS+GEOHR2_R+PACADPAI+ALCADTYP+FAV_LRV1, weight=wTSA_S, data=newdata, fami $7 y=$ =poisson)HR2_R+PACADPAI+ALCADTYP+FAV_LRV1, weight=WTSA_S, dat warning mes sage: $y=$ poisson)

> summary (mode18c, dispersion=mode $18 \mathrm{c} \$ d e v i a n c e /$ mode $18 \mathrm{c} \$ \mathrm{df}$. residua 1 )

Ca11:

glm(formula = ACUTE_VS BMI_4CAT + CRNC_TOT + DHHA_SEX + DHHA_AGE + EDUADH04 + INCADIA5 + INST_NEW1 + SMKA_202 + DHHA_MS + GEOHR2 R + PACADPAI + ALCADTYP + FAV_LRV1, fami $7 y=$ poisson, data = newdata, weights $=$ WTSA_S)

Coefficients:

(Intercept)

BMI_4CAToverweight

BMI_4CATobese

BMI_4CATmorbid obesity

CRNC_TOT

DHHA_SEXmale

DHHA_AGE

EDUADH04SECONDARY GRAD.

EDUADH04OTHER POST-SEC.

EDUADHO4POST-SEC. GRAD.

EDUADHO4NOT STATED

INCADIA5LOWER MIDDLE

INCADIA5MIDDLE

INCADIA5UPPER MIDDLE

INCADIA5HIGHEST

INCADIA5NOT STATED

INST_NEW1Eastern

INST_NEW1Nor thern

INST_NEWIWestern

SMKA_2020CCASIONALLY

SMKA_202NOT AT ALL

DHHA_MSCOMMON-LAW

DHHA_MSWIDOWED

DHHA_MSSEPARATED

DHHA_MSDIVORCED

DHHA MSSINGLE

GEOHR2_Rurban

PACADPAIMODERATE

PACADPAI INACTIVE

PACADPAINOT STATED

ALCADTYPOCC. DRINKER

ALCADTYPFORMER DRINKER

ALCADTYPNEVER DRANK

0.437050
servings per day -0.099278

$\begin{array}{lr}\text { ALCADTYPNEVER DRANK } & 0.437050 \\ \text { FAV_LRV } 1<5 \text { serVings per day }-0.099278\end{array}$

---

Signif. codes: 0 'th' 0.001

Estimate

$-0.597965$

$-0.044114$

$-0.235328$

0.153535

0.237892

$-0.434086$

$-0.009114$

$-0.004344$

0.045573

0.133307

$-0.985173$

$-0.037131$

$-0.342040$

$-0.243522$

$-0.693028$

$-0.807401$

$-0.365076$

0.570850

0.030954

$-0.223153$

0.081530

0.066064

$-0.355746$

0.499317

0.122545

0.239611

$-0.103884$

$-0.095713$

0.134724

0.471432

0.364798

0.247734

Std. Error
0.331703
0.099196
0.124093
0.150855
0.022732
0.094238
0.004456
0.148756
0.195908
0.121851
0.993231
0.181309
0.177346
0.179395
0.228355
0.280412
0.113320
0.123625
0.115384
0.272810
0.098805
0.143962
0.328969
0.288406
0.252512
0.132677
0.087969
0.157664
0.133745
0.228909
0.105096
0.129675
0.142128
0.097334

$z$ value $\operatorname{Pr}(>|z|)$

-1.8030 .071433 .

$\begin{array}{lll}-0.445 & 0.656525\end{array}$

$-1.8960 .057910$

1.0180 .308790

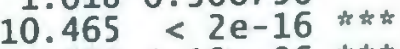

$-4.6064 .10 \mathrm{e}-06$ *t

$\begin{array}{lll}-2.045 & 0.040844 \text { * }\end{array}$

$-0.0290 .976703$

0.2330 .816054

1.0940 .273947

$\begin{array}{lll}-0.992 & 0.321253\end{array}$

$-0.2050 .837735$

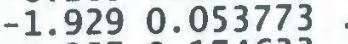

$-1.3570 .174633$

$-3.0350 .002406$

-2.8790 .003985 * *

-3.2220 .001275 *t

$4.6183 .88 \mathrm{e}-06$ **

0.2680 .788489

$\begin{array}{lll}-0.818 & 0.413369\end{array}$

0.8250 .409279

0.4590 .646306

$\begin{array}{lll}-1.081 & 0.279520\end{array}$

1.7310 .083398

0.4850 .627461

1.8060 .070923

$\begin{array}{lll}-1.181 & 0.237638\end{array}$

$-0.6070 .543805$

1.0070 .313781

2.0590 .039449 *

3.4710 .000518 *स

1.9100 .056078

3.0750 .002105 को कै

$-1.0200 .307745$

(Dispersion parameter for poisson family taken to be 136.1035 )

Page 9 
poisson_reg_utilization.txt

Number of Fisher scoring iterations: 6

MODEL4A: ACTUAL VISITS LOS (BMI ONLY)

$>$ mode $7<-g 7 m$ (ACUTE_LO BMI_4CAT+DHHA_SEX+DHHA_AGE+EDUADH04+INCADIA5+INST_NEW1+SMKA_202 mode $74<-g 1 m$ (ACUTE_LO BMI_4CATHA_MS+GEOHR2_R+PACADPAI+ALCADTYP+FAV_LRV1, weight=WTSA_S, data=newdata, fami $1 y=$ poisson)

Ca77:

glm Cformula = ACUTE_LO BMI_4CAT + DHHA_SEX + DHHA_AGE + EDUADH04 + INCADIA5 + INST_NEW1 + SMKA_202 + DHHA_MS + GEOHR2_R + PACADPAI + ALCADTYP + FAV_LRV1, famiTy = poisson, data = newdata, weights = WTSA_S)

\section{Coefficients:}

(Intercept)

BMI_4CAToverweight

BMI_4CATobese

BMI_4CATmorbid obesity

DHHA_SEXmale

DHHA_AGE

EDUADH04SECONDARY GRAD.

EDUADH04OTHER POST-SEC.

EDUADH04POST-SEC. GRAD .

EDUADH04NOT STATED

INCADIA5LOWER MIDDLE

INCADIA5MIDDLE

INCADIASUPPER MIDDLE

INCADIA5HIGHEST

INCADIASNOT STATED

INST_NEWIEastern

INST_NEW1Northern

INST_NEWIWestern

SMKA_2020CCASIONALLY

SMKA_202NOT AT ALL

DHHA_MSCOMMON - LAW

DHHA_MSWIDOWED

DHHA_MSSEPARATED

DHHA_MSDIVORCED

DHHA_MSSINGLE

GEOHR 2_Rurban

PACADPAIMODERATE

PACADPAIINACTIVE

PACADPAINOT STATED

ALCADTYPOCC. DRINKER

ALCADTYPFORMER DRINKER

ALCADTYPNEVER DRANK

FAV_LRV $1<5$ servings per day

signif. codes: $0^{\prime \prime k} \times 10.001$

signif. codes: 0 'sk' 0.001

(Dispersion parameter for poisson family taken to be 1114.435 )

\begin{tabular}{|c|c|c|c|c|}
\hline $\begin{array}{r}\text { Estimate } \\
-1.066287 \\
-0.203312 \\
-0.629870 \\
-0.205329 \\
-0.677907 \\
0.037086 \\
0.282306 \\
0.077378 \\
0.461396 \\
-1.555127 \\
-0.796619 \\
-1.063631 \\
-1.011284 \\
-2.035191 \\
-1.994241 \\
-0.140038 \\
0.911028 \\
0.036488 \\
-0.176175 \\
0.083637 \\
0.838739 \\
-0.459901 \\
0.642423 \\
0.621599 \\
1.242737 \\
0.083080 \\
0.236582 \\
0.790910 \\
2.055568 \\
0.328181 \\
0.286058 \\
0.812373 \\
0.108310\end{array}$ & $\begin{array}{c}\text { td. Error } \\
0.395346 \\
0.112430 \\
0.154618 \\
0.190021 \\
0.112435 \\
0.004814 \\
0.161296 \\
0.244620 \\
0.137956 \\
1.860592 \\
0.168110 \\
0.153486 \\
0.160124 \\
0.278716 \\
0.347470 \\
0.134833 \\
0.143628 \\
0.139805 \\
0.300855 \\
0.114175 \\
0.153602 \\
0.354941 \\
0.346772 \\
0.242960 \\
0.143543 \\
0.102510 \\
0.227273 \\
0.194292 \\
0.240179 \\
0.124490 \\
0.157939 \\
0.150668 \\
0.121470\end{array}$ & $\begin{array}{r}-2.697 \\
-1.808 \\
-4.074 \\
-1.081 \\
-6.029 \\
7.703 \\
1.750 \\
0.316 \\
3.345 \\
-0.836 \\
-4.739 \\
-6.930 \\
-6.316 \\
-7.302 \\
-5.739 \\
-1.039 \\
6.343 \\
0.261 \\
-0.586 \\
0.733 \\
5.460 \\
-1.296 \\
1.853 \\
2.558 \\
8.658 \\
0.810 \\
1.041 \\
4.071 \\
8.558 \\
2.636 \\
1.811 \\
5.392 \\
0.892\end{array}$ & $\begin{array}{l}0.006995 \\
0.070553 \\
4.63 \mathrm{e}-05 \\
0.279893 \\
1.65 \mathrm{e}-09 \\
1.33 \mathrm{e}-14 \\
0.080077 \\
0.751759 \\
0.000824 \\
0.403254 \\
2.15 \mathrm{e}-06 \\
4.21 \mathrm{e}-12 \\
2.69 \mathrm{e}-10 \\
2.83 \mathrm{e}-13 \\
9.51 \mathrm{e}-09 \\
0.298988 \\
2.25 \mathrm{e}-10 \\
0.794097 \\
0.558157 \\
0.463841 \\
4.75 \mathrm{e}-08 \\
0.195075 \\
0.063942 \\
0.010514 \\
<2 \mathrm{e}-16 \\
0.417677 \\
0.297893 \\
4.69 \mathrm{e}-05 \\
22 \mathrm{e}-16 \\
0.008384 \\
0.070110 \\
6.98 \mathrm{e}-08 \\
0.372576\end{array}$ & 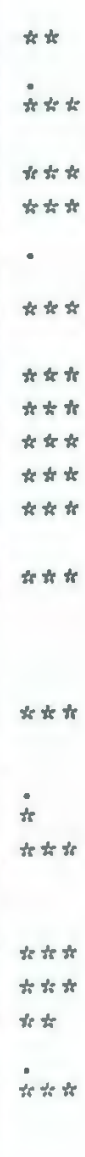 \\
\hline
\end{tabular}

Estimate std. Error $z$ value $\operatorname{Pr}(>|z|)$ $0.395346-2.697 \quad 0.006995$

0.112430

0.154618

0.112435

0.004814

0.161296

0.137956

1.860592

0.168110

. 160124

0.278716

0.347470

0.143628

0.139805

0.300855

0.114175

0.354941

0.346772

0.242960

0.102510

0.227273

0.240179

0.124490

0.150668

0.121470 *t

at

$\star *$

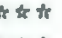

$+\pi$

t女

tक th t t t

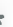
to tot tक स * it

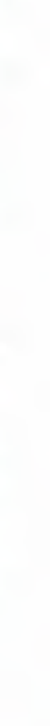

\footnotetext{
Page 10
} 
poisson_reg_utilization.txt

MODEL4B: ACTUAL ACUTE LOS (CC ONLY)

$========-$

$>$

mode $14 b<-g 7 m$ (ACUTE_LO CRNC_TOT+DHHA_SEX+DHHA_AGE+EDUADH04+INCADIA5+INST_NEW1+SMKA_20 2+DHHA_MS+GEOHR2_R+PACADPAI+ALCADTYP+FAV_LRV1, weight=WTSA_S, data=newdata, fami $7 y=$ poisson)

Ca17:

glm (formula = ACUTE_LO CRNC_TOT + DHHA_SEX + DHHA_AGE + EDUADHO $4+$ INCADIA5 + INST_NEW1 + SMKA_202 + DHHA_MS + GEOHR2_R + PACADPAI + ALCADTYP + FAV_LRV1, family = poisson, data = newdata, weights = WTSA_S )

coefficients:

\section{(Intercept) \\ CRNC_TOT \\ DHHA_SEXma Te}

DHHA_AGE

EDUADH04SECONDARY GRAD.

EDUADH04OTHER POST-SEC.

EDUADH04POST-SEC. GRAD.

EDUADHO4NOT STATED

INCADIA5LOWER MIDDLE

INCADIA5MIDDLE

INCADIA5UPPER MIDDLE

INCADIASHIGHEST

INCADIASNOT STATED

INST_NEW1Eastern

INST_NEW1Nor thern

INST_NEWIWestern

SMKA_2020CCASIONALLY

SMKA_202NOT AT ALL

DHHA_MSCOMMON - LAW

DHHA_MSWIDOWED

DHHA_MSSEPARATED

DHHA_MSDIVORCED

DHHA_MSSINGLE

GEOHR2_Rurban

PACADPAIMODERATE

PACADPAIINACTIVE

PACADPAINOT STATED

ALCADTYPOCC. DRINKER

ALCADTYPFORMER DRINKER

ALCADTYPNEVER DRANK

FAV_LRVI< 5 servings per day
Estimate std.

$-1.127294$

0.301845

$-0.604013$

0.024167

0.421262

$-0.012976$

0.454333

$-1.654119$

$-0.905971$

$-1.004355$

$-0.837389$

$-1.817744$

$-1.889484$

$-0.094693$

0.883524

0.161006

0.017599

0.078301

0.848022

$-0.336366$

0.415221

0.608895

1.234416

0.053605

0.087360

0.648442

1.785045

0.234478

0.067594

0.672358

0.075712 td. Error $z$ value $\operatorname{Pr}(>|z|)$

$0.386370-2.9180 .003527$ क स

$0.02404212 .555<2 \mathrm{e}-16$ t*

$0.109400 \quad-5.5213 .37 \mathrm{e}-08$ tक t

0.004998

0.156629

0.236489

0.133570

1.795511

0.165141

0.148320

0.154847

0.269143

0.335531

0.130497

0.139104

0.136355

0.291522

0.110484

0.147328

0.343218

0.340451

0.234736

0.143686

0.099066

0.219208

0.187822

0.234278

0.120865

0.152936

0.145066

0.117363

$4.8351 .33 \mathrm{e}-06$ 채

2.6900 .007155 * *

$-0.0550 .956241$

3.4010 .000670 *t

$-0.9210 .356919$

$-5.4864 .11 e-08$ tक को

$-6.7721 .27 e-11$ tक

$-5.4086 .38 \mathrm{e}-08$ *t

$-6.7541 .44 e-11$ 中⿰ 幺

$-5.6311 .79 \mathrm{e}-08$ को को

$-0.7260 .468062$

$6.3522 .13 \mathrm{e}-10$ t*

1.1810 .237686

0.0600 .951860

$0.709 \quad 0.478504$

$5.7568 .61 \mathrm{e}-09$ tक

$\begin{array}{lll}-0.980 & 0.327069\end{array}$

1.2200 .222609

2.5940 .009488 * *

$8.591<2 e-16$ th t

0.5410 .588436

0.3990 .690242

3.4520 .000556 * *

$7.6192 .55 \mathrm{e}-14$ th th

1.9400 .052381 .

$0.442 \quad 0.658507$

$4.6353 .57 \mathrm{e}-06 * \pi *$

0.6450 .518859

signif. codes: 0 k* 0.001 '* 0.01 " 0.05 ' 0.1 ' 1

(Dispersion parameter for poisson family taken to be 1038.505)

Number of Fisher scoring iterations: 7

Page 11 
poisson_reg_utilization.txt

MODEL4C: ACTUAL ACUTE LOS (BMI + CC)

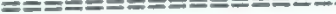

$>$

(14C-g) 1+SMKA_202+DHHA_MS+GEOHR2_R+PACADPAI+ALCADTYP+FAV_LRV1, weight=WTSA_S, data=newdata, family=poisson)

> summary(mode19c, dispersion=mode19c\$deviance/mode19c\$df, residual)

glm (formula = ACUTE_LO BMI_4CAT + CRNC_TOT + DHHA_SEX + DHHA_AGE + EDUADH04 + INCADIA5 + INST_NEW1 + SMKA_202 + DHHA_MS + GEOHR2 R +
PACADPAI + ALCADTYP + FAV_LRV1, family = poisson, data = newdata, weights $=$ WTSA_S )

coefficients:

(Intercept)

BMI_4CATOverweight

BMI_4CATobese

BMI_4CATmorbid obesity

CRNC_TOT

DHHA_SEXmale

DHHA_AGE

EDUADH04SECONDARY GRAD.

EDUADH04OTHER POST-SEC.

EDUADH04POST-SEC. GRAD.

EDUADH04NOT STATED

INCADIASLOWER MIDDLE

INCADIASMIDDLE

INCADIASUPPER MIDDLE

INCADIASHIGHEST

INCADIA5NOT STATED

INST_NEW1Eastern

INST_NEW1Northern

INST NEWIWestern

SMKA_2020CCASIONALLY

SMKA_202NOT AT ALL

DHHA_MSCOMMON-LAW

DHHA_MSWIDOWED

DHHA_MSSEPARATED

DHHA_MSDIVORCED

DHHA_MSSINGLE

GEOHR2_RUrban

PACADPAIMODERATE

PACADPAIINACTIVE

PACADPAINOT STATED

ALCADTYPOCC. DRINKER

ALCADTYPFORMER DRINKER

ALCADTYPNEVER DRANK

FAV_LRV1< 5 servings per day
Estimate std. Error $z$ value $\operatorname{Pr}(>|z|)$

- 1.025232

$-0.182979$

$-0.649896$

$-0.280339$

0.305881

$-0.533563$

0.023623

0.418586

$-0.037582$

0.442093

$-1.528528$

$-0.883201$

$-0.970571$

$-0.817781$

$-1.798047$

$-1.869330$

$-0.100377$

0.916886

0.136858

0.028413

0.116477

0.815991

$-0.275395$

0.355318

0.604929

1.222225

0.029781

0.147409

0.675981

1.737475

0.264606

0.149843

0.698121

0.092908
0.390524

0.109013

0.149617

0.185092

0.024262

0.110222

0.005026

0.156585

0.236290

0.133121

1.785793

0.166937

0.149998

0.155483

0.268609

0.333622

0.131088

0.139085

0.136458

0.290358

0.112035

0.147372

0.341794

0.343759

0.233636

0.144444

0.099585

0.218650

0.187144

0.234102

0.119960

0.154376

0.144891

0.117113 $\begin{array}{lll}-1.515 & 0.129877\end{array}$
-2.6250 .008658 क क स

$\begin{array}{lll}-1.679 & 0.093248\end{array}$

$-4.3441 .40 \mathrm{e}-05$ को को

$12.608<2 \mathrm{e}-16$ औ*

-4.841 1.29e-06 th

$4.7002 .60 \mathrm{e}-06$ का

2.6730 .007513 * *

$-0.1590 .873629$

3.3210 .000897 추

$-0.8560 .392032$

$-5.2911 .22 \mathrm{e}-07$ \#

$-6.4719 .76 \mathrm{e}-11$ th

$-5.2601 .44 e-07$ *t

$-6.6942 .17 e-11$ tक

- $5.6032 .10 \mathrm{e}-08$ *t

$-0.7660 .443845$

$6.5924 .33 \mathrm{e}-11$ *t*

1.0030 .315893

$0.098 \quad 0.922046$

1.0400 .298506

$5.5373 .08 \mathrm{e}-08$ tt

$-0.8060 .420397$

1.0340 .301311

2.5890 .009620 th th

$8.462<2 \mathrm{e}-16$ * का th

0.2990 .764899

0.6740 .500199

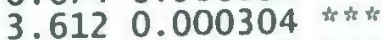

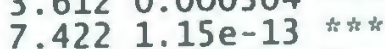

2.2060 .027399 *

0.9710 .331728

$4.8181 .45 \mathrm{e}-06$ tw

0.7930 .427591

signif. codes: 0 'ton' 0.001

$1 \ldots 0.01$

'*'0.05' 0.1 ' 1

(Dispersion parameter for poisson family taken to be 1026.980 )

Number of Fisher scoring iterations: 7 
poisson_reg_utilization.txt

MODEL5A: ACTUAL ACUTE SOC (BMI ONLY)

$>$

mode $15 \mathrm{a}<-\mathrm{g} 1 \mathrm{~m}$ (SDC_VSTS BMI_4CAT+DHHA_SEX+DHHA_AGE+EDUADH04+INCADIA5+INST_NEW1+SMKA_20 2+DHHA_MS+GEOHR2_R+PACADPAI+ALCADTYP+FAV_LRV1, weight=WTSA_S, data=newdata, fami $1 \mathrm{y}=$ poisson)

Ca11:

glm(formula $=$ SDC_VSTS $~$ BMI_4CAT + DHHA_SEX + DHHA_AGE + EDUADHO4 + INCADIA5 + INST_NEW1 + SMKA_202 + DHHA_MS + GEOHR2_R + PACADPAI + ALCADTYP + FAV_LRV1, family = poisson, data $=$ newdata, weights = WTSA_S )

coefficients:

(Intercept)

BMI_4CATOverweight

BMI_4CATobese

BMI_4CATmorbid obesity

DHHA_SEXmale

DHHA_AGE

EDUADHO4SECONDARY GRAD.

EDUADHO4OTHER POST-SEC.

EDUADH04POST-SEC. GRAD. EDUADHO4NOT STATED

INCADIA5LOWER MIDDLE

INCADIA5MIDDLE

INCADIASUPPER MIDDLE

INCADIASHIGHEST

INCADIA 5NOT STATED

INST_NEW1Eastern

INST_NEW1Northern

INST_NEW1Western

SMKA_2020CCASIONALLY

SMKA_202NOT AT ALL

DHHA_MSCOMMON-LAW

DHHA_MSWIDOWED

DHHA_MSSEPARATED

DHHA_MSDIVORCED

DHHA_MSSINGLE

GEOHR2_Rurban

PACADPAIMODERATE

PACADPAIINACTIVE

PACADPAINOT STATED

ALCADTYPOCC. DRINKER

ALCADTYPFORMER DRINKER

ALCADTYPNEVER DRANK

ALCADTYPNEVER DRAN 5 servings per day -0.033320

Estimate
-2.088341
0.260275
0.228591
0.001941
-0.547964
0.021738
-0.177872
-0.200807
-0.150481
0.862524
0.471479
0.239356
0.173757
0.295517
0.234946
0.189150
-0.098127
-0.269842
-1.083640
-0.043794
0.164881
-0.676355
0.478001
-0.167100
-0.093595
-0.073788
0.050583
0.152617
-0.526497
0.239273
0.143169
0.054640
-0.033320
0

std

Error

0.375291

0.105382

0.123769

0.200568

0.097939

0.004496

0.148792

0.234112

0.123176

0.431359

0.231475

0.222836

0.228640

0.254188

0.320919

0.106230

0.163583

0.131607

0.411389

0.102842

0.152373

0.326530

0.311847

0.294855

0.162438

0.090533

0.164509

0.143973

0.386358

0.110765

0.132007

0.167755

0.103811 $z$ value $\operatorname{Pr}(>|z|)$

$-5.5652 .63 e-08$ औt

2.4700 .01352 *

1.8470 .06476 .

$0.010 \quad 0.99228$

$-5.5952 .21 e-08$ के औ

$4.8351 .33 \mathrm{e}-06$ to th

$\begin{array}{ll}-1.195 & 0.23192\end{array}$

$\begin{array}{ll}-0.858 & 0.39103\end{array}$

$\begin{array}{ll}-1.222 & 0.22183\end{array}$

2.0000 .04555 *

2.0370 .04167 *

$\begin{array}{ll}1.074 & 0.28276\end{array}$

$0.760 \quad 0.44728$

$\begin{array}{ll}1.163 & 0.24500\end{array}$

0.7320 .46411

1.7810 .07498 .

$-0.600 \quad 0.54860$

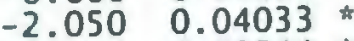

-2.6340 .00844 *

$\begin{array}{ll}-0.426 & 0.67023\end{array}$

$\begin{array}{ll}1.082 & 0.27921\end{array}$

$-2.071$

0.03833 *

$\begin{array}{ll}1.533 & 0.12532\end{array}$

$\begin{array}{ll}-0.567 & 0.57091\end{array}$

$\begin{array}{ll}-0.576 & 0.56449\end{array}$

$\begin{array}{ll}-0.815 & 0.41505\end{array}$

$0.307 \quad 0.75848$

$\begin{array}{ll}1.060 & 0.28912\end{array}$

$\begin{array}{ll}-1.363 & 0.17297\end{array}$

2.1600 .03076 *

$\begin{array}{ll}1.085 & 0.27812\end{array}$

$0.326 \quad 0.74464$

$-0.3210 .74824$

Signif. codes: 0 'ktk' 0.001

*' 0.05 ' 0.1 ' 1

(Dispersion parameter for poisson family taken to be 123.019 )

Number of Fisher Scoring iterations: 6

Page 13 
poisson_reg_utilization.txt

MODEL5B: ACTUAL ACUTE SDC (CC ONLY)

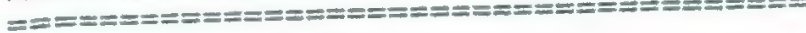

$>$

mode $15 b<-g 1 m$ (SDC_VSTS CRNC_TOT+DHHA_SEX+DHHA_AGE+EDUADH04+INCADIA5+INST_NEW1+SMKA_20 2+DHHA_MS+GEOHR2_R+PACADPAI +ALCADTYP+FAV_LRV1, weight=WTSA_S, data=newdata, fami $7 y=$ poisson)

$>$ summary (mode110b, dispersion=mode110b\$deviance/mode110b\$df.residual) ca71:

glm (formula = SDC_VSTS CRNC_TOT + DHHA_SEX + DHHA_AGE + EDUADHO4 + INCADIA5 + INST_NEW1 + SMKA_202 + DHHA_MS + GEOHR2_R + PACADPAI + ALCADTYP + FAV_LRV1, family $=$ poisson, data $=$ newdata, weights $=$ WTSA_S $)$

Coefficients:

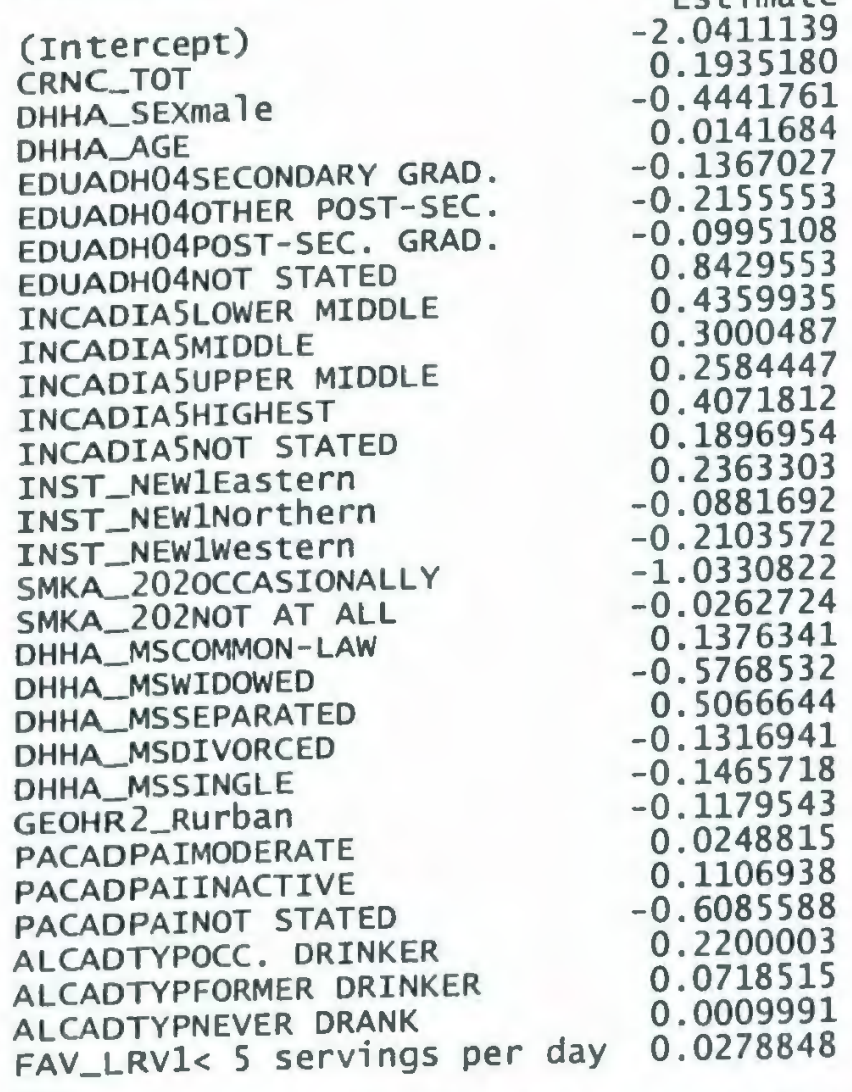
std. Error $z$ value $\operatorname{pr}(>|z|)$
0.3704344
0.0235414
0.0959302
0.0046302
0.1472525
0.2311582
0.1217675
0.4250432
0.2281097
0.2193108
0.2246040
0.2492520
0.3144755
0.1047445
0.1613208
0.1300899
0.4048501
0.1014758
0.1492647
0.3209179
0.3060526
0.2904878
0.1614577
0.0892717
0.1620869
0.1421834
0.3804542
0.1087140
0.1307840
0.1656270
0.1025146
$-5.510$
$8.220<2 \mathrm{e}-16$
$3.65 e-06$ **
$-4.6303 .65 \mathrm{e}-06$
$-0.9280 .35322$
$-0.933$
$-0.817$
1.983
1.911
1.368
1.151
1.634
0.603
2.256
$-0.547$
$-1.617$
$-2.552$
$-0.259$
0.922
$-1.798$
1.655
$-0.453$
$-0.908$
$-1.321$
0.154
0.779
$-1.600$
2.024
0.549
0.006
0.272
0.35108
0.41380
0.04734 *
0.05596 .
0.17127
0.24987
0.10234
0.54637
0.02405 *
0.58469
0.10588
0.01072 *
0.79571
0.35649
0.07225
0.09783 .
0.65029
0.36398
0.18640
0.87800
0.43626
0.10970
0.04300 *
0.58274
0.99519
0.78562

signif. codes: 0 'kt* 0.001 '*t' 0.01 " 0.05 ' 0.1 ' 1

(Dispersion parameter for poisson family taken to be 119.0912 )

Number of Fisher scoring iterations: 6 
poisson_reg_utilization.txt

MODELSC: ACTUAL VISITS SDC (BMI \& CC)

de $15 \mathrm{C}<-97 \mathrm{~m}$ (SDC VSTS BMI 4CAT+CRNC_TOT+DHHA_SEX+DHHA_AGE+EDUADH04+INCADIA5+INST_NEW 1+SMKA_202+DHHA_MS+GEOHR2_R+PACADPAI +ALCADTYP+FAV_LRV1, weight=WTSA_S, data=newdata, fami $7 y=$ poi sson)

Ca 11 :

glm (formula $=$ SDC_VSTS BMI_4CAT + CRNC_TOT + DHHA_SEX + DHHA_AGE + EDUADH04 + INCADIA5 + INST_NEW1 + SMKA_202 + DHHA_MS + GEOHR2 R +
PACADPAI + ALCADTYP + FAV_LRV1, family = poisson, data = newdata, weights $=$ WTSA_S )

coefficients:

(Intercept)

BMI_4CAToverweight

BMI_4CATobese

BMI_4CATmorbid obesity

CRNC_TOT

DHHA_SEXmale

DHHA_AGE

EDUADH04SECONDARY GRAD.

EDUADHO4OTHER POST-SEC.

EDUADH04POST-SEC. GRAD .

EDUADH04NOT STATED

INCADIASLOWER MIDDLE

INCADIA 5MIDOLE

INCADIASUPPER MIDDLE

INCADIASHIGHEST

INCADIASNOT STATED

INST_NEWIEastern

INST_NEW1Northern

INST_NEWIWestern

SMKA_2020CCASIONALLY

SMKA_202NOT AT ALL

DHHA MSCOMMON-LAW

DHHA_MSWIDOWED

DHHA_MSSEPARATED

DHHA_MSDIVORCED

DHHA MSS INGLE

GEOHR2_.RUrban

PACADPAIMODERATE

PACADPAIINACTIVE

PACADPAINOT STATED

ALCADTYPOCC. DRINKER

ALCADTYPFORMER DRINKER

ALCADTYPNEVER DRANK

FAV_LRV $1<5$ servings per day

Signif. codes: 0 * 0.001

Estimate std.

$-2.112285$

0.179969

$-0.149755$

0.197743

$-0.480412$

0.013371

$-0.132188$

$-0.213092$

$-0.105059$

0.804322

0.426611

0.298540

0.244423

0.382826

0.206323

0.249581

$-0.090600$

$-0.200668$

$-1.032102$

$-0.017381$

0.149434

$-0.562798$

0.530918

$-0.098383$

$-0.120039$

$-0.112997$

$-0.009222$

0.097549

$-0.609303$

0.212545

0.079393

$-0.008837$

0.015530
0.371316
0.103586
0.122092
0.199077
0.023774
0.096837
0.004638
0.146942
0.230696
0.121792
0.424827
0.228094
0.219397
0.224560
0.249406
0.314052
0.105123
0.161128
0.130165
0.404312
0.102024
0.149234
0.320389
0.305692
0.290466
0.161738
0.089152
0.162077
0.141917
0.380189
0.108772
0.130618
0.165311
0.102541

$z$ value $\operatorname{Pr}(>|z|)$

$-5.6891 .28 \mathrm{e}-08$ और

2.5210 .01171 *

$\begin{array}{ll}1.474 & 0.14047\end{array}$

0.45190

$8.318<2 e-16$ और

$<2 e-16$ *

$-4.961$

$7.01 e-07$

2.883

0.00394 * *

$-0.900$

0.36834

$-0.924$

0.35565

$-0.8630 .38835$

1.8930 .05832 .

1.8700 .06144

1.3610 .17360

$1.088 \quad 0.27640$

$\begin{array}{ll}1.535 & 0.12480\end{array}$

0.6570 .51120

2.3740 .01759 *

$-0.562$

$-1.5420 .12316$

0.57392

$\begin{array}{ll}-2.553 & 0.01069\end{array}$

$\begin{array}{ll}-0.170 & 0.86472\end{array}$

1.0010 .31666

$-1.757$

1.737

0.07898 .

0.08243

$\begin{array}{ll}-0.742 & 0.45798\end{array}$

$\begin{array}{ll}-1.267 & 0.20499\end{array}$

$-0.0570 .95462$

0.6870 .49185

$-1.603$

0.10902

1.954

0.05070 .

0.608

$-0.053$

0.151

0.54331

0.95737

0.87962

(Dispersion parameter for poisson family taken to be 118.6257 )

Number of Fisher scoring iterations: 6 


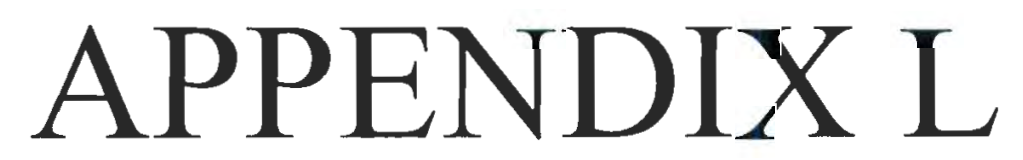

\section{Multiple Regression Models 6a-7c Physician and Hospital Costs}


poisson_reg_costs.txt

Model 6a: MULTIPLE REGRESSION ON TOTAL DOCTOR COSTS-TOTAL VISITS (BMI ON, , ISITS $>0$

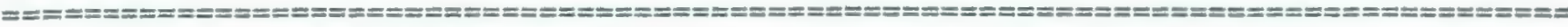

$>$ model14a<-1m(LOGTOT_FFS BMI_4CAT+DHHA_SEX + DHHA_AGE +EDUADH04.NA+INST_NEW1 + SMKA_202+GEOHR2_R, data $=$ newdata, subset $=$ LOGTOT_FFS $>0$, weights $=$ WTSA_S, na.action=na.omit)

Ca11:1m(formula $=$ LOGTOT_FFS BMI_4CAT + DHHA_SEX + DHHA_AGE + EDUADH04.NA + INST_NEW1 + SMKA_202 + GEOHR2_R, data $=$ newdata, subset $=$ LOGTOT_FFS > 0 , weights $=$ WTSA_S, na.action $=$ na.omit)

coefficients:

(Intercept)

BMI_4CAToverweight

BMI_4CATobese

BMI_4CATmorbid obesity
Estimate std. Error $t$ value $\operatorname{Pr}(>|t|)$
$5.1494620 .17559829 .325<2 \mathrm{e}-16$ t*
0.043761
0.066607
0.439183
0.077528
0.093166
0.564
0.57253
0.139658
3.145
0.47476
0.00169 * *
$\begin{array}{rr}0.066399 & -11.268 \\ 0.002853 & 9.451\end{array}$
0.115933
0.172329
0.094643
$-1.577$
0.234
$-0.026$
$-0.837$
0.121487
$-7.310$
0.155
0.090315
0.181922
$-0.473$
$\begin{array}{ll}0.073555 & 1.144 \\ 0.068216 & 2.911\end{array}$
$<2 e-16 * *$
$<2 e-16$
0.11503
0.11503
0.97945
0.40298
$4.32 e-13$ * *
0.87665
0.63639
0.25293
0.00366 t

0.084124

signif. codes: 0 "*t $0.001 * * 1^{\prime} 0.01$ "*' 0.05

Multiple R-Squared: $0.1808, \quad$ Adjusted R-squared: 0.1732

F-statistic: 23.76 on 14 and 1507 DF, p-value: < 2.2e-16 
poisson_reg_costs.txt

Model 6b: MULTIPLE REGRESSION ON TOTAL DOCTOR COSTS- TOTAL VISITS (CHRONIC CONDITIONS ONLY) VISITS $>0$

-

$>$ mode $76 b<-7 m$ (LOGTOT_FFS CRNC_TOT+DHHA_SEX + DHHA_AGE +EDUADH04.NA+INST_NEWI + SMKA_202+GEOHR2_R, data=newdata, subset=LOGTOT_FFS $>0$, weightS $=$ WTSA_S, na.action=na.omit)

Ca11:7m(formula = LOGTOT_FFS CRNC_TOT + DHHA_SEX + DHHA_AGE + EDUADHO4.NA + INST_NEW1 + SMKA_202 + GEOHR2_R, data = newdata, subset $=$ LOGTOT_FFS $>$ 0 , weights $=$ WTSA_S, na.action = na.omit)

coefficients:

(Intercept)

Estimate std. Error $t$ value $\operatorname{Pr}(>|t|)$

CRNC_TOT 5.070464

0.163113

31.086

$<2 \mathrm{e}-16 * *$

CRNC_TOT

0.302874

0.022353

13.549

$<2 e-16$

DHHA_SEXma Te

$-0.640994$

0.062833

$-10.202$

$<2 \mathrm{e}-16$ kt $*$

DHHA_AGE

0.017291

EDUADH04. NASECONDARY GRAD. -0.082666

EDUADH04. NAOTHER POST-SEC. 0.022233

EDUADH04. NAPOST-SEC. GRAD.

INST_NEW1Eastern

INST_NEW1Nor thern

0.085686

$-0.029087$

$-0.925441$

INST_NEWIWestern

0.055346

SMKA_202OCCASIONALLY

$-0.004298$

SMKA_202NOT AT ALL

0.123581

0.002772

0.109983

6.237

5. $76 e-10$ to fo

GEOHR2_Rurban

0.187339

0.163108

$-0.752$

0.45239

0.089696

0.077840

0.114960

0.085130

0.136

0.89160

0.955

0.33958

$-0.374$

0.70870

$-8.0501 .66 e-15 *$

0.172333

0.650

0.51570

0.069319

$-0.025$

0.064391

1.783

2.909

0.98010

---

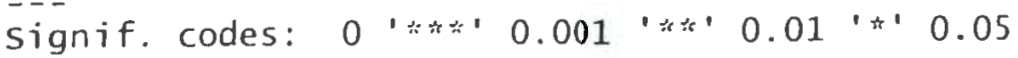

Multiple R-squared: $0.2648, \quad$ Adjusted R-squared: 0.259

F-statistic: 45.3 on 12 and 1509 DF, p-value: < $2.2 \mathrm{e}-16$ 
poisson_reg_costs.txt

Model 6C: MULTIPLE REGRESSION ON TOTAL DOCTOR COSTS TOTAL VISITS (BMI \& CHRONIC CONDITIONS )VISITS $>0$

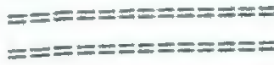

$>$ mode 16C<-1m(LOGTOT_FFS BMI_4CAT+CRNC_TOT+DHHA_SEX + DHHA_AGE +EDUADH04. NA+INST_NEWI +

SMKA_202+GEOHR2_R, data=newdata, subset=LOGTOT_FFS $>0$, weightS = WTSA_S, na.action=na.omit)

$1 \mathrm{~m}$ (formula $=$ LOGTOT_FFS $\sim$ BMI_4CAT + CRNC_TOT + DHHA_SEX + DHHA_AGE + EDUADH04.NA + INST_NEW1 + SMKA_202 + GEOHR2_R, data = newdata, subset $=$ LOGTOT_FFS $>0$, weights $=$ WTSA_S, na.action $=$ na.omit)

\section{coefficients:}

(Intercept)

BMI_4CAToverweight

BMI_4CATobese

BMI_4CATmorbid obesity

CRNC_TOT

DHHA_SEXma $7 \mathrm{e}$

DHHA_AGE

EDUADH04. NASECONDARY GRAD.

EDUADH04. NAOTHER POST-SEC.

EDUADH04.NAPOST-SEC. GRAD.

INST_NEW1Eastern

INST_NEWINorthern

INST_NEWIWestern

SMKA_2020CCASIONALLY

SMKA_202NOT AT ALL

GEOHR2_RUrban

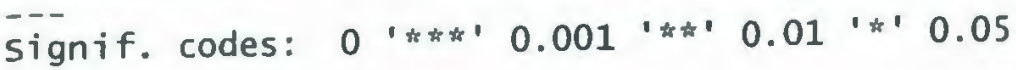

Multiple R-Squared: 0.2672 , Adjusted R-squared: 0.2599

F-statistic: 36.61 on 15 and 1506 DF, p-value: $<2.2 \mathrm{e}-16$

$-0.0165636$
Estimate Std. Error $t$ value $\operatorname{Pr}(>|t|)$ $5.02068210 .166419330 .169<2 \mathrm{e}-16$ *t
0.0413052
0.0014855
0.2826877
0.0733515
0.563
0.57344
0.0882950
$-0.017$
0.98658
0.1326548
2.131
0.03325 क
0.2992767
0.0224629
13.323
$<2 \mathrm{e}-16$ **t

$-0.6361334$

0.0175635

$-0.0776993$

0.0274680
0.0977544

$-0.9167431$

0.0663751
-0.0001326

0.1142107

$0.0633825-10.036$

0.0027901

6.295

0.1099710

$-0.707$

0.1630474

0.168

1.088

0.0781841

$-0.212$

0.1149621

$-9.974$

0.0855393

$-0.001$

1.640

2.803

$<2 \mathrm{e}-16$ *t

$4.03 e-10$ और

0.47996

0.86624

0.27683

0.83225

$3.01 e-15$ tक स

0.1722424

0.43789

0.99939

0.10116

0.00513 *

\footnotetext{
F-statistic: 36.61 on 15 and 1 bo 0 ,
} 
poisson_reg_costs.txt

MODEL 7A: MULTIPLE REGRESSION ON TOTAL HOSPITAL COSTS - IN-PATIENT VISITS ONLY (BMI ONLY)

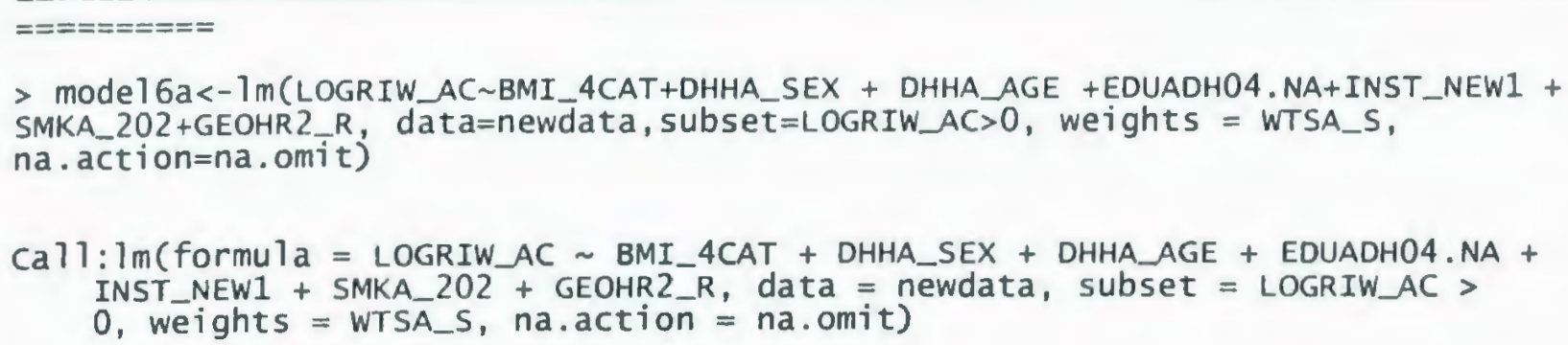

Coefficients:

Estimate std. Error $t$ value $\operatorname{Pr}(>|t|)$

$\begin{array}{lrrrr}\text { (Intercept) } & 8.036485 & 0.210350 & 38.205<2 \mathrm{e}-16 \text { *** } \\ & 0.011373 & 0.096806 & 0.117 & 0.906532 \\ \text { BMI_4CAToverweight } & -0.198899 & 0.120334 & -1.653 & 0.099085 \\ \text { BMI_4CATobese } & 0.178410 & 0.150510 & 1.185 & 0.236527\end{array}$

\begin{tabular}{|c|c|c|c|}
\hline $\begin{array}{l}\text { DHHA_SEXmaTe } \\
\text { DHHA_AGE } \\
\text { EDUADHO4.NASECONDARY GRAD. } \\
\text { EDUADH04.NAOTHER POST-SEC. } \\
\text { EDUADH04.NAPOST-SEC. GRAD. } \\
\text { INST_NEWIEAStern } \\
\text { INST_NEWINOrthern } \\
\text { INST_NEWIWEStern } \\
\text { SMKA_202OCCASIONALLY } \\
\text { SMKA_202NOT AT ALL } \\
\text { GEOHR2_RURban }\end{array}$ & $\begin{array}{r}-0.012913 \\
0.013474 \\
0.186879 \\
0.053696 \\
0.103637 \\
-0.039464 \\
0.079018 \\
0.005885 \\
-0.013897 \\
-0.052659 \\
-0.164849\end{array}$ & $\begin{array}{l}0.090027 \\
0.003443 \\
0.144892 \\
0.189311 \\
0.109832 \\
0.107392 \\
0.134382 \\
0.108645 \\
0.244503 \\
0.092439 \\
0.085837\end{array}$ & $\begin{array}{rl}-0.143 & 0.886018 \\
3.913 & 0.000106 \\
1.290 & 0.197821 \\
0.284 & 0.776823 \\
0.944 & 0.345906 \\
-0.367 & 0.713447 \\
0.588 & 0.556835 \\
0.054 & 0.956827 \\
-0.057 & 0.954701 \\
-0.570 & 0.569201 \\
-1.920 & 0.055459\end{array}$ \\
\hline
\end{tabular}

Signif. codes: $0 * * * 0.001 * * 0.01 * 1^{\prime} 0.05$

Residual standard error: 8.643 on 429 degrees of freedom

(110 observations deleted due to missingness)

Multiple R-Squared: 0.05625 , Adjusted R-squared: 0.02545

F-statistic: 1.826 on 14 and 429 DF, p-value: 0.03284 
poisson_reg_costs.txt

MODEL 78: MULTIPLE REGRESSION ON TOTAL HOSPITAL COSTS - IN-PATIENT VISITS ONLY (CHRONIC CONDITIONS ONLY)

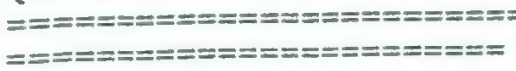

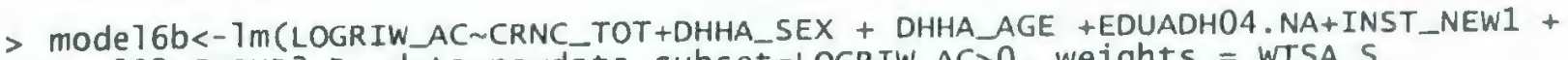
SMKA_202+GEOHR2_R, data $=$ newdata, subset $=$ LOGRIW_AC $>0$, weights $=$ WTSA_S, na. action=na. omit)

Ca11:1m(formula = LOGRIW_AC CRNC_TOT + DHHA_SEX + DHHA_AGE + EDUADH04.NA + INST_NEW1 + SMKA_202 + GEOHR2_R, data $=$ newdata, subset $=$ LOGRIW_AC > 0 , weights $=$ WTSA_S, na.action $=$ na.omit)

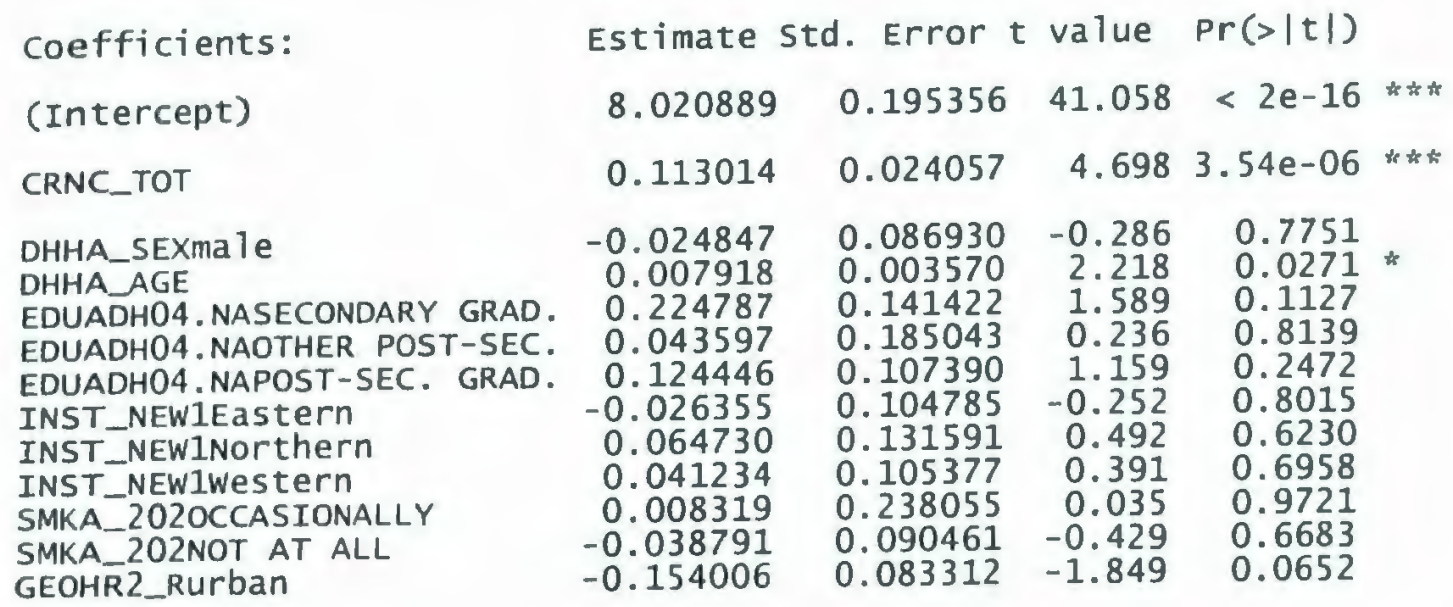

-.-

signif. codes: $0^{\prime * * *} 0.001^{\prime * *} 0.01 * 1^{\prime} 0.05$

Multiple R-Squared: 0.09016, Adjusted R-squared: 0.06482

F-statistic: 3.559 on 12 and 431 DF, p-value: $4.556 \mathrm{e}-05$ 
poisson_reg_costs.txt

MODEL 7C: MULTIPLE REGRESSION ON TOTAL HOSPITAL COSTS - IN-PATIENT VISITS ONLY (BMI \& CHRONIC CONDITOINS)

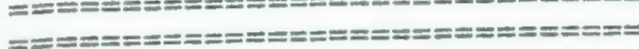

$>$ mode16c<-7m(LOGRIW_AC BMI_4CAT+CRNC_TOT+DHHA_SEX + DHHA_AGE +EDUADH04. NA+INST_NEW1 + SMKA_202+GEOHR2_R, data=newdata, subset=LOGRIW_AC>0, weights = WTSA_S, na. action=na. omit)

Ca11:7m(formu 1a = LOGRIW_AC BMI_4CAT + CRNC_TOT + DHHA_SEX + DHHA_AGE + EDUADH04.NA + INST_NEW1 + SMKA_202 + GEOHR2_R, data = newdata, subset $=$ LOGRIW_AC $>0$, weights $=$ WTSA_S, na.action $=$ na.omit)

Coefficients:

Estimate std. Error $t$ value $\operatorname{Pr}(>|t|)$

\begin{tabular}{|c|c|c|c|c|}
\hline (Intercept) & 8.0495155 & 0.2053895 & 39.191 & $<2 \mathrm{e}-16$ \\
\hline $\begin{array}{l}\text { BMI_4CAToverweight } \\
\text { BMI_4CATobese } \\
\text { BMI_4CATmorbid obesity }\end{array}$ & $\begin{array}{r}0.0001471 \\
-0.2269788 \\
0.1175686\end{array}$ & $\begin{array}{l}0.0945447 \\
0.1176376 \\
0.1475178\end{array}$ & $\begin{array}{r}0.002 \\
-1.929 \\
0.797\end{array}$ & $\begin{array}{l}0.9988 \\
0.0543 \\
0.4259\end{array}$ \\
\hline RNC_TOT & 0.1130989 & 0.0240835 & 4.696 & $3.58 \mathrm{e}-06$ \\
\hline $\begin{array}{l}\text { DHHA_SEXma le } \\
\text { DHHA_AGE } \\
\text { EDUADH04. NASECONDARY GRAD. } \\
\text { EDUADH04.NAOTHER POST-SEC. } \\
\text { EDUADH04.NAPOST-SEC. GRAD. } \\
\text { INST_NEW1EaStern } \\
\text { INST_NEW1NOrthern } \\
\text { INST_NEW1WeStern } \\
\text { SMKA_2O2OCCASIONALLY } \\
\text { SMKA_202NOT AT ALL } \\
\text { GEOHR2_RURban }\end{array}$ & $\begin{array}{r}0.0038326 \\
0.0079246 \\
0.2660297 \\
0.0458323 \\
0.1411636 \\
-0.0391718 \\
0.0688345 \\
0.0331522 \\
0.0127589 \\
-0.0442212 \\
-0.1829029\end{array}$ & $\begin{array}{l}0.0879681 \\
0.0035632 \\
0.1424630 \\
0.1848377 \\
0.1075294 \\
0.1048498 \\
0.1312189 \\
0.1062321 \\
0.2387835 \\
0.0902688 \\
0.0838931\end{array}$ & $\begin{array}{r}0.044 \\
2.224 \\
1.867 \\
0.248 \\
1.313 \\
-0.374 \\
0.525 \\
0.312 \\
0.053 \\
-0.490 \\
-2.180\end{array}$ & $\begin{array}{l}0.9653 \\
0.0267 \\
0.0625 \\
0.8043 \\
0.1900 \\
0.7089 \\
0.6001 \\
0.7551 \\
0.9574 \\
0.6245 \\
0.0298\end{array}$ \\
\hline
\end{tabular}

- -

signif. codes: $0^{\prime * k} 0.001$ "*k' 0.01 '*' 0.05 ' 0.1 ' 1

Multiple R-Squared: $0.1025, \quad$ Adjusted R-squared: 0.07104

F-statistic: 3.258 on 15 and 428 DF, p-value: $3.716 e-05$
} 




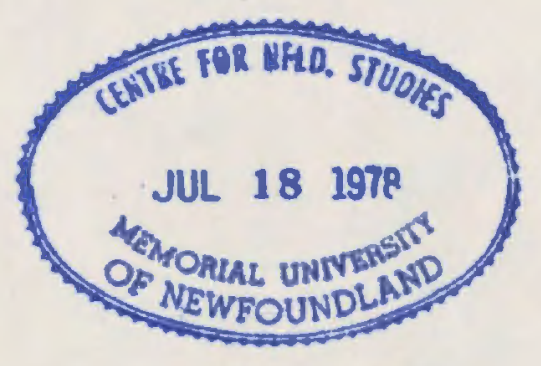


